UNIVERSIDAD DE SALAMANCA

Facultad de Ciencias Químicas

Departamento de Química Analítica, Nutrición y Bromatología

\title{
DESARROLLO DE NUEVAS ESTRATEGIAS ANALÍTICAS PARA LA DETERMINACIÓN DE COMPUESTOS DE INTERÉS BIOLÓGICO Y MEDIOAMBIENTAL
}

DEVELOPMENT OF NEW ANALYTICAL STRATEGIES FOR THE DETERMINATION OF COMPOUNDS OF BIOLOGICAL AND ENVIRONMENTAL INTEREST

Rosa María González Paredes 2017 



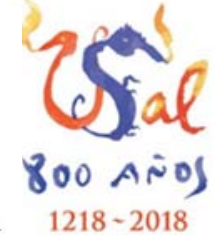

UNIVERSIDAD DE SALAMANCA

FACULTAD DE CIENCIAS QUÍMICAS

Departamento de Química Analítica, Nutrición y Bromatología

\section{DESARROLLO DE NUEVAS \\ ESTRATEGIAS ANALÍTICAS PARA LA \\ DETERMINACIÓN DE COMPUESTOS DE \\ INTERÉS BIOLÓGICO Y \\ MEDIOAMBIENTAL}

DEVELOPMENT OF NEW ANALYTICAL

STRATEGIES FOR THE DETERMINATION OF

COMPOUNDS OF BIOLOGICAL AND

ENVIRONMENTAL INTEREST

ROSA MARÍA GONZÁLEZ PAREDES

2017 



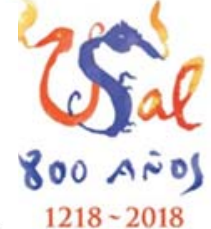

UNIVERSIDAD DE SALAMANCA

FACULTAD DE CIENCIAS QUÍMICAS

Departamento de Química Analítica, Nutrición y Bromatología

\section{DESARROLLO DE NUEVAS ESTRATEGIAS ANALÍTICAS PARA LA DETERMINACIÓN DE COMPUESTOS DE INTERÉS BIOLÓGICO Y MEDIOAMBIENTAL} DEVELOPMENT OF NEW ANALYTICAL STRATEGIES FOR THE DETERMINATION OF COMPOUNDS OF BIOLOGICAL AND ENVIRONMENTAL INTEREST

Memoria que para optar al Grado de Doctor por la Universidad de Salamanca presenta la licenciada Rosa María González Paredes.

Salamanca, 21 de abril de 2017

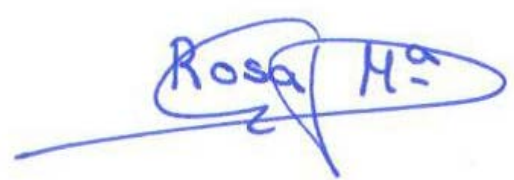

Fdo.: Rosa María González Paredes 

D. Bernardo Moreno Cordero, Catedrático de Química Analítica de la Universidad de Salamanca, D. José Luis Pérez Pavón, Catedrático de Química Analítica de la Universidad de Salamanca, y D. Carmelo García Pinto, Profesor Titular de Química Analítica de la Universidad de Salamanca, directores del trabajo "Desarrollo de nuevas estrategias analíticas para la determinación de compuestos de interés biológico y medioambiental", realizado por la licenciada Rosa María González Paredes para optar al grado de Doctor por la Universidad de Salamanca, autorizan la presentación del mismo en el formato de compendio de artículos al considerar que se han alcanzado los objetivos inicialmente previstos.

Salamanca, 21 de abril de 2017

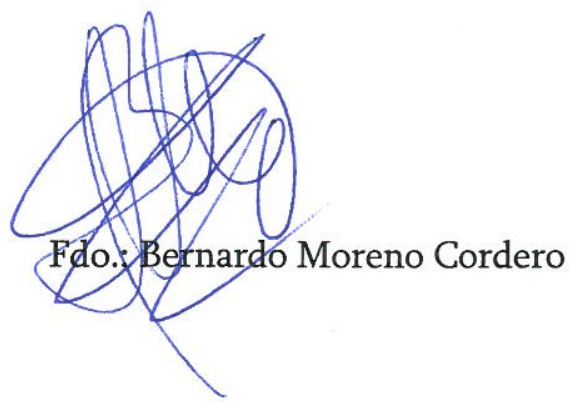

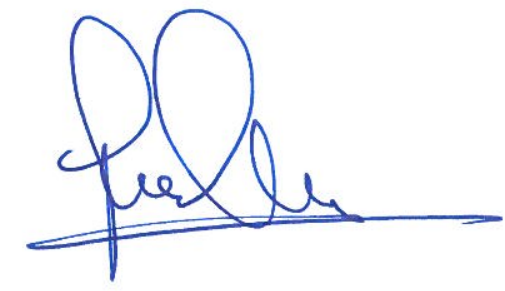

Fdo.: José Luis Pérez Pavón



Fdo.: Carmelo García Pinto 

Deseo expresar mi agradecimiento a la Universidad de Salamanca por la concesión de una beca para la Formación de Personal Investigador financiadas por el Banco Santander (Convocatoria 2011) y por la ayuda adicional otorgada dentro del Programa de Movilidad de Personal Investigador-Modalidad Ib para la realización de una estancia breve en un centro de investigación extranjero.

El trabajo realizado ha sido financiado por los proyectos: CTQ201017514/BQU del Ministerio de Ciencia e Innovación; CTQ2013-47993-P/BQU del Ministerio de Economía y Competitividad; y SA-162U14 y Grupo de Excelencia GR87 de la Junta de Castilla y León.

El proyecto SILEP (Supelco Ionic Evaluation Program) de la casa comercial Supelco a través de Sigma-Aldrich proporcionó las columnas de líquidos iónicos con las que se realizó parte del trabajo recogido en esta memoria. 

Deseo expresar mi más sincero agradecimiento a los directores de este trabajo, Bernardo Moreno Cordero, José Luis Pérez Pavón y Carmelo García Pinto por su entrega, dedicación y apoyo constante.

A todos los miembros del Departamento de Química Analítica, Nutrición y Bromatología por tratarme con tanto cariño y cercanía, y por ayudarme en cualquier asunto siempre que lo he necesitado.

A todos mis compañeros de laboratorio con los que he compartido momentos inolvidables, tanto a los que han estado, como a los que están, porque sin duda todos ellos han contribuido a mi formación investigadora y docente con sus numerosos consejos, pero sobre todo a mi formación personal con sus inagotables ánimos y su inquebrantable amistad.

De todos y cada uno de los miembros de este Departamento, desde el sótano hasta el ático, me llevo un grato recuerdo, puesto que todos, de una forma u otra, me habéis aportado algo y habéis dejado una huella imborrable en mí. Todos formáis parte de una experiencia magnífica que me acompañará allá donde vaya.

No quisiera terminar estos escuetos, pero no por ello menos sentidos agradecimientos, sin dedicar unas palabras a mi familia y amigos, por acompañarme en cada uno de los pasos que voy dando en la vida sin cuestionarme ni juzgarme, simplemente haciéndome sentir que siempre están y estarán a mi lado. 

I would like to thank Prof. Dr. Bogusław Buszewski for the opportunity to work in the Department of Environmental Chemistry and Bioanalytics of the Nicolaus Copernicus University (Toruń, Poland) and for his support, kindness and hospitality during my research stay.

My most sincere thanks to Dr. Tomasz Ligor for his invaluable help, support and friendliness all the time.

I am also very grateful to all personal of the laboratory for their hospitality and affection.

Thanks to all of you, this stay has been an unforgettable experience for me. 

Esta Tesis Doctoral se presenta como compendio de las publicaciones que se especifican a continuación:

1. "Ionic liquids as stationary phases in gas chromatography: Determination of chlorobenzenes in soils" Rosa María González Paredes, Carmelo García Pinto, José Luis Pérez Pavón, Bernardo Moreno Cordero.

Journal of Separation Science 37 (2014) 1448-1455.

2. "In situ derivatization combined to automated microextraction by packed sorbents for the determination of chlorophenols in soil samples by gas chromatography mass spectrometry"

Rosa María González Paredes, Carmelo García Pinto, José Luis Pérez Pavón, Bernardo Moreno Cordero.

Journal of Chromatography A 1359 (2014) 52-59.

3. "Derivatization coupled to headspace programmed-temperature vaporizer gas chromatography with mass spectrometry for the determination of amino acids: Application to urine samples" Rosa María González Paredes, Carmelo García Pinto, José Luis Pérez Pavón, Bernardo Moreno Cordero. Journal of Separation Science 39 (2016) 3375-3383. 
4. "Determination of possible biomarkers in urine samples by a rapid method based on headspace-gas chromatography-mass spectrometry"

Rosa María González Paredes, Carmelo García Pinto, José Luis Pérez Pavón, Bernardo Moreno Cordero.

Enviado para su publicación

Departamento de Química Analítica, Nutrición y Bromatología. Facultad de Ciencias Químicas, Universidad de Salamanca, España. 
ÍNDICE 

INTRODUCCIÓN GENERAL....................................... 5

1. ESTRUCTURA Y OBJETO DE LA TESIS....................... 7

1.1. Estructura de la Tesis.................................. 9

1.2. Introducción.......................................... 9

1.3. Objetivos e hipótesis de trabajo...................... 15

1.4. Metodología........................................... 17

2. TÉCNICAS DE PRETRATAMIENTO DE MUESTRA............. 21

2.1. QuEChERS........................................ 23

2.2. Microextracción con sorbentes empaquetados............ 41

2.3. Reacciones de derivatización.......................... 59

2.3.1. Reacción de acilación de fenoles................. $\quad 59$

2.3.2. Derivatización de aminoácidos con cloroformiatos................................... 63

2.4. Generación de espacio de cabeza....................... 79

3. LÍQUIDOS IÓNICOS EN CROMATOGRAFÍA DE GASES....... 97

4. CONFIGURACIONES INSTRUMENTALES..................... 109

4.1. Esquema de las configuraciones instrumentales utilizadas.

4.2. Sistema de introducción de muestras-PTV-GC- $\mu$ ECD.... 113

4.2.1. Sistema de introducción de muestras.............. 113

4.2.2. Inyector de temperatura programada............. 113

4.2.3. Cromatógrafo de gases............................ 114

4.2.4. Micro-detector de captura electrónica............. 117 
4.3. Sistema de introducción de muestras-PTV-GC-MS....... 118

4.3.1. Sistema de introducción de muestras............... 118

4.3.2. Inyector de temperatura programada............. 119

4.3.3. Cromatógrafo de gases........................... 119

4.3.4. Espectrómetro de masas........................... 119

CAPÍTULO I. DESARROLLO DE METODOLOGÍAS ANALÍTICAS PARA LA DETERMINACIÓN DE COMPUESTOS DE INTERÉS MEDIOAMBIENTAL EN MUESTRAS DE SUELO.......................... 121

I.1. Líquidos iónicos como fases estacionarias en cromatografía de gases. Determinación de clorobencenos en suelos.

Artículo de investigación I.1.

- Resumen..........................................

- Journal of Separation Science 37 (2014) 1448-1455.

I.2. Derivatización in situ combinada con MEPS para la determinación de clorofenoles en suelos mediante GC-MS.

Artículo de investigación I.2.

- Resumen.

- Journal of Chromatography A 1359 (2014) 52-59 ...

CAPÍTULO II. DESARROLLO DE METODOLOGÍAS ANALÍTICAS PARA LA DETERMINACIÓN DE COMPUESTOS DE INTERÉS BIOLÓGICO EN MUESTRAS DE ORINA.

II.1. Derivatización acoplada a HS-PTV-GC-MS para la determinación de aminoácidos. Aplicación a muestras de orina.

Artículo de investigación II.1.

- Resumen.

- Journal of Separation Science 39 (2016) 3375-3383. 
II.2. Determinación de posibles biomarcadores en muestras de orina mediante un método rápido basado en HS-GC-MS.

Artículo de investigación II.2.

-Resumen....................................... 205

- Enviado para publicación.......................... 213

CONCLUSIONES................................................. 241

VERSIÓN RESUMIDA EN INGLÉS / SUMMARY IN ENGLISH.......... 249

Apéndice: MENCIÓN “DOCTORADO INTERNACIONAL”.............. 267 



\section{SIGLAS Y ACRÓNIMOS / ACRONYMS}

\begin{tabular}{|c|c|}
\hline \multirow[t]{2}{*}{ ANOVA } & Análisis de varianza \\
\hline & Analysis of variance \\
\hline \multirow[t]{2}{*}{$\mathrm{AOAC}$} & Asociación de Comunidades Analíticas \\
\hline & Association of Analytical Communities \\
\hline \multirow[t]{2}{*}{ BTEX } & Benceno, tolueno, etilbenceno y xileno \\
\hline & Benzene, toluene, ethylbenzene and xylene \\
\hline \multirow[t]{2}{*}{$\mathrm{CE}$} & Electroforesis capilar \\
\hline & Capillary electrophoresis \\
\hline \multirow[t]{2}{*}{$d-S P E$} & Extracción en fase sólida dispersiva \\
\hline & Dispersive solid phase extraction \\
\hline \multirow[t]{2}{*}{ DBS } & Mancha de sangre seca \\
\hline & Dried blood spot \\
\hline \multirow[t]{2}{*}{ DPS } & Mancha de plasma seco \\
\hline & Dried plasma spot \\
\hline \multirow[t]{2}{*}{ DUS } & Mancha de orina seca \\
\hline & Dried urine spot \\
\hline \multirow[t]{2}{*}{$\mu$-ECD } & Micro-detector de captura electrónica \\
\hline & Micro-electron capture detector \\
\hline \multirow[t]{2}{*}{ ECF } & Cloroformiato de etilo \\
\hline & Ethyl chloroformate \\
\hline \multirow[t]{2}{*}{ GC } & Cromatografía de gases \\
\hline & Gas chromatography \\
\hline
\end{tabular}




\begin{tabular}{|c|c|}
\hline GCB & Negro de carbón grafitizado \\
\hline & Graphitized carbon black \\
\hline GC x GC & Cromatografía multidimensional \\
\hline & Comprehensive two dimensional gas chromatography \\
\hline HS & Generación de espacio de cabeza \\
\hline & Headspace sampling \\
\hline IL & Líquido iónico \\
\hline & Ionic liquid \\
\hline LLE & Extracción líquido-líquido \\
\hline & Liquid-liquid extraction \\
\hline LLME & Microextracción líquido-líquido \\
\hline & Liquid-liquid microextraction \\
\hline LOD & Límite de detección \\
\hline & Limit of detection \\
\hline LOQ & Límite de cuantificación \\
\hline & Limit of quantification \\
\hline LPME & Microextración en fase líquida \\
\hline & Liquid-phase microextraction \\
\hline MEPS & Microextracción con sorbentes empaquetados \\
\hline & Microextraction by packed sorbent \\
\hline MIP & Polímero de impresión molecular \\
\hline & Molecularly imprinted polymer \\
\hline MRM & Método multi-residuo \\
\hline & Multiresidue method \\
\hline
\end{tabular}


MS Espectrometría de masas

Mass spectrometry

PAHs Hidrocarburos policíclicos aromáticos

Polycyclic aromatic hydrocarbons

PSA Amina primaria-secundaria

Primary secondary amine

P\&T Purga y trampa

Purge and trap

PTV Inyector de temperatura programada

Programmed temperature vaporizer

QuEChERS Rápido, sencillo, barato, eficaz, robusto y seguro

Quick, easy, cheap, efficient, rugged and safe

RAM Material de acceso restringido

Restricted access material

RSD Desviación estándar relativa

Relative standard deviation

SALLE Extracción líquido-líquido asistida por sales

Salting-out assisted liquid-liquid extraction

SHS Generación de espacio de cabeza estático

Static headspace sampling

SIM Seguimiento de iones seleccionados

Selected ion monitoring

SME Microextracción con disolventes

Solvent microextraction 
SPE $\quad$ Extracción en fase sólida

Solid-phase extraction

SPME Microextracción en fase sólida

Solid-phase microextraction

USEPA Agenda de Protección Medioambiental de Estados Unidos

United States Environmental Protection Agency

VOCs Compuestos orgánicos volátiles

Volatile organic compounds 
INTRODUCCIÓN GENERAL 



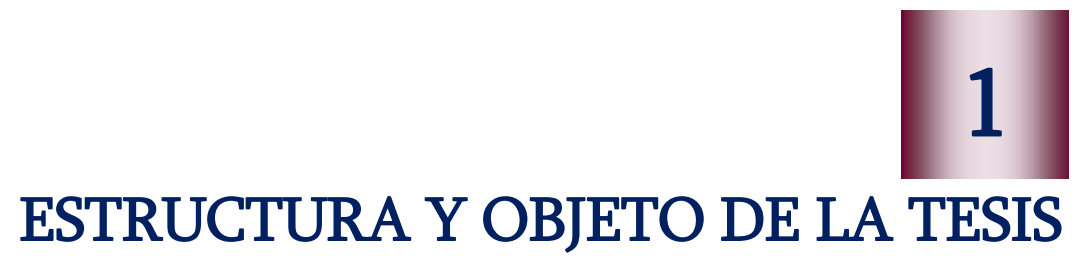





\subsection{Estructura de la Tesis}

A fin de cumplir con el formato requerido para la presentación de esta Tesis Doctoral como compendio de publicaciones, esta memoria se ha estructurado en varias secciones. La primera de ellas es una introducción general en la que se pone de manifiesto la coherencia y relación directa entre las publicaciones presentadas, así como los objetivos y la metodología seguidos. Además, en esta introducción general también se describen las técnicas empleadas de pretratamiento de muestra, el uso de los líquidos iónicos en cromatografía de gases y las configuraciones instrumentales.

Las secciones siguientes son dos capítulos, en cada uno de los cuales se incluyen dos publicaciones. Para cada uno de los cuatro trabajos realizados se presenta un resumen en el que se especifican los objetivos de la investigación, la metodología utilizada, los resultados alcanzados y las conclusiones finales, seguido de una copia de la publicación original.

Finalmente, se exponen las conclusiones generales de las cuatro publicaciones incluidas en esta Tesis Doctoral.

\subsection{Introducción}

La Tesis Doctoral que se presenta se centra en el desarrollo de metodologías analíticas basadas en la utilización de la cromatografía de gases (gas chromatography, GC) como técnica de separación y encaminadas a conseguir una reducción significativa del esfuerzo necesario para llevar a 
cabo la etapa de tratamiento de muestra con el fin de minimizar costes y tiempo y reducir los errores asociados a esta etapa del proceso analítico.

La Tesis consta de dos partes dedicadas ambas a la puesta a punto de metodologías que cumplan las características anteriormente descritas pero que difieren en el tipo de matriz de estudio, dedicándose la primera parte a muestras de suelo y la segunda a matrices biológicas.

La primera parte se centra en el desarrollo de metodologías de análisis para la determinación de contaminantes en muestras de suelos, concretamente de clorobencenos y clorofenoles, mientras que la segunda parte está dedicada al desarrollo de metodologías rápidas y sencillas para la detección y cuantificación en orina de compuestos de interés biológico como son los aminoácidos y algunos compuestos descritos como posibles biomarcadores.

En todas las metodologías propuestas se utiliza un inyector de temperatura programada (programmed temperature vaporizer, PTV) para la transferencia de la muestra al sistema cromatográfico.

Teniendo en cuenta la diversidad del tipo de matrices y de la naturaleza de los compuestos estudiados, la optimización y desarrollo de métodos analíticos rápidos para su determinación implica el uso de una gran variedad de técnicas de pretratamiento de muestra.

Para el primer trabajo de determinación de clorobencenos en suelos incluido en la primera parte de la Tesis, se optimiza su extracción de la matriz mediante una versión simplificada de QuEChERS (Quick, easy, cheap, efficient, rugged and safe) ya descrita [1]. Esta versión elimina la etapa de 
limpieza mediante extracción en fase sólida dispersiva (dispersive solid phase extraction, d-SPE) debido a las propiedades no grasas de las matrices de suelos. Además, en este trabajo, también se lleva a cabo una evaluación del comportamiento de las nuevas columnas capilares con fases estacionarias de líquidos iónicos para la separación cromatográfica de los clorobencenos. La investigación con líquidos iónicos ha experimentado un gran crecimiento en los últimos años y algunas de las especiales características que presentan [2] los han hecho interesantes para su estudio como fases estacionarias en cromatografía de gases. Estos estudios se realizaron con distintas columnas de líquidos iónicos suministradas por la casa Supelco a través de Sigma-Aldrich (Steinheim, Alemania) dentro del proyecto internacional SILEP (Supelco ionic liquid evaluation program).

Por otro lado, para la determinación de clorofenoles en suelos se realiza la preconcentración de los mismos mediante un sistema automático de microextracción con sorbentes empaquetados (microextraction by packed sorbent, MEPS). La técnica MEPS surge del intento de agilizar y hacer más eficientes las técnicas de extracción ya existentes [3,4], basándose en la miniaturización y automatización del proceso de extracción en fase sólida convencional (solid phase extraction, SPE), de forma que se utilizan menores volúmenes de disolventes y, además, los cartuchos de extracción se pueden reutilizar un gran número de veces [5].

En cromatografía de gases son extensamente utilizadas las reacciones de derivatización para la mejora de las características cromatográficas de algunos compuestos. Éste es el caso de los clorofenoles, para los que se utiliza una reacción de acilación con anhídrido acético en medio básico 
directamente en el suelo. Esta derivatización en medio acuoso favorece en gran medida el acoplamiento con la técnica MEPS que se aplica posteriormente.

Las muestras biológicas son una fuente de información valiosa sobre el estado de salud de un individuo ya que la presencia de ciertos compuestos orgánicos en ellas está relacionada con algunas funciones celulares básicas [6]. Es por ello que, el desarrollo de nuevos métodos analíticos para la determinación de compuestos en muestras biológicas, tal y como se contempla en la segunda parte de la Tesis, constituye una línea de estudio necesaria y de gran interés no sólo para la investigación y descubrimiento de nuevos biomarcadores [7,8] que puedan servir para la detección precoz de algunas afecciones, sino también como herramienta para evaluar la utilidad de aquellos que ya han sido propuestos.

En el primer trabajo de esta segunda parte de la Tesis, en el que se propone un método para la determinación de aminoácidos en muestras de orina, se utiliza de nuevo una reacción de derivatización. En este caso se emplea el cloroformiato de etilo (ethyl chloroformate, ECF) como reactivo de derivatización de los aminoácidos. Esta derivatización está acoplada a la técnica de espacio de cabeza (headspace sampling, HS), de forma que los compuestos derivatizados volátiles se inyectan directamente en el sistema cromatográfico sin necesidad de llevar a cabo tratamientos adicionales para extraerlos de la matriz.

En el segundo trabajo que se incluye en esta parte de la Tesis, se sigue utilizando la generación de espacio de cabeza como técnica de pretratamiento para la determinación en muestras de orina de compuestos 
pertenecientes a diferentes familias químicas y con diferentes volatilidades que se han descrito como posibles biomarcadores. La técnica de generación de espacio de cabeza para el análisis de biomarcadores volátiles en matrices biológicas no sólo minimiza el tratamiento que se debe hacer a este tipo de muestras [9] y permite la automatización del proceso, sino que, además, reduce las interferencias de compuestos no volátiles presentes en matrices complejas [10]. De esta forma se consigue un método rápido, simple y sensible capaz de determinar compuestos con diferentes grupos funcionales que se puede aplicar al análisis de un gran número de muestras para el descubrimiento y confirmación de biomarcadores.

\section{Bibliografía}

[1] C. García Pinto, M.E. Fernández Laespada, S. Herrero Martín, A.M. Casas Ferreira, J.L. Pérez Pavón, B. Moreno Cordero, Simplified QuEChERS approach for the extraction of chlorinated compounds from soil samples, Talanta 81 (2010) 385-391.

[2] C.F. Poole, S.K. Poole, Ionic Liquid stationary phases for gas chromatography, J. Sep. Sci. 34 (2011) 888-900.

[3] M. Abdel-Rehim, New trend in sample preparation: on-line microextraction in packed syringe for liquid and gas chromatography applications. I. Determination of local anaesthetics in human plasma samples using gas chromatography-mass spectrometry, J. Chromatogr. B 801 (2004) 317-321. 
[4] A. El-Beqqali, A. Kussak, M. Abdel-Rehim, Fast and sensitive environmental analysis utilizing microextraction in packed syringe online with gas chromatography-mass spectrometry: determination of polycyclic aromatic hydrocarbons in water, J. Chromatogr. A 1114 (2006) 234-238.

[5] A.R. Chaves, F.Z. Leandro, J.A. Carris, M.E.C. Queiroz, Microextraction in packed sorbent for analysis of antidepressants in human plasma by liquid chromatography and spectrophotometric detection, J. Chromatogr. B 878 (2010) 2123-2129.

[6] I. Horváth, Z. Lázár, N. Gyulai, M. Kollai, G. Losonczy, Exhaled biomarkers in lung cancer, Eur. Respir. J. 34 (2009) 261-275.

[7] H.J. Issaq, T.J. Waybright, T.D. Veenstra, Cancer biomarker discovery: Opportunities and pitfalls in analytical methods, Electrophoresis 32 (2011) 967-975.

[8] K.A. Kouremenos, M. Johansson, P.J. Marriott, Advances in gas chromatographic methods for the identification of biomarkers in cancer, J. Cancer 3 (2012) 404-420.

[9] B. Kolb, in I.D. Wilson (Editor), CHROMATOGRAPHY: GAS | Headspace Gas Chromatography. Encyclopedia of Separation Science. Academic Press, Oxford, 2000, pp. 489-496.

[10] N.H. Snow, G.P. Bullock, Novel techniques for enhancing sensitivity in static headspace extraction-gas chromatography, J. Chromatogr. A 1217 (2010) 2726-2735. 


\subsection{Objetivos e hipótesis de trabajo}

El objetivo general y principal de esta Tesis Doctoral es la puesta a punto de metodologías analíticas rápidas en las que se simplifique el tratamiento de muestra y que permitan la determinación y cuantificación de diferentes compuestos de interés biológico y medioambiental en distintos tipos de matrices.

Los objetivos concretos de la primera parte dedicada a la determinación de contaminantes en suelos son fundamentalmente:

$>$ El estudio de las ventajas que puedan tener las nuevas fases estacionarias de líquidos iónicos para la determinación de clorobencenos.

$>$ El estudio de las posibilidades analíticas del acoplamiento de la derivatización y extracción in situ con MEPS para la preconcentración y determinación de clorofenoles.

Las hipótesis que se plantean en esta primera parte son:

- La aplicabilidad de una versión simplificada de QuEChERS para la extracción de los clorobencenos de las muestras de suelos.

- La posibilidad de que las nuevas fases estacionarias de líquidos iónicos, más polares que las convencionales, permitan la separación adecuada de los clorobencenos, teniendo en cuenta que en función del líquido iónico que constituya la fase estacionaria, las características cromatográficas serán diferentes. 
- La posibilidad de llevar a cabo la derivatización y extracción de los clorofenoles in situ en el suelo.

- La viabilidad de la técnica de microextracción MEPS para la preconcentración de los clorofenoles una vez optimizadas todas las variables involucradas en ella.

- La posibilidad de acoplamiento de la derivatización y extracción in situ con MEPS para la determinación de clorofenoles.

Los objetivos concretos de la segunda parte de la Tesis dedicada a matrices biológicas son:

El desarrollo de una serie de metodologías analíticas rápidas, sensibles y eficaces que puedan ser aplicables a la determinación y cuantificación en orina de algunos compuestos descritos como biomarcadores.

El estudio de la técnica de espacio de cabeza para la determinación de compuestos volátiles en orina.

El estudio del acoplamiento de la derivatización y generación de espacio de cabeza para la determinación de aminoácidos.

En esta parte, las hipótesis de trabajo planteadas son:

- La posibilidad de llevar a cabo la derivatización de los aminoácidos en muestras de orina.

- La posibilidad de acoplamiento de la derivatización con la generación de espacio de cabeza para la determinación de aminoácidos. 
- La adecuación de la técnica de espacio de cabeza para la determinación de compuestos volátiles en orina, reduciendo así el tratamiento que debe hacerse a la muestra y eliminando muchos compuestos interferentes no volátiles que presenta este tipo de matriz.

\subsection{Metodología}

Los trabajos de investigación realizados y dirigidos al cumplimiento de los objetivos y a la evaluación de las hipótesis planteadas para cada una de las partes de esta Tesis son:

Determinación de compuestos de interés medioambiental en muestras de suelos

- Puesta a punto de un método analítico para la determinación de clorobencenos en suelos mediante la extracción basada en una versión simplificada de QuEChERS, la separación cromatográfica de los analitos mediante diferentes columnas capilares de líquidos iónicos y la detección mediante micro-detector de captura electrónica (micro-electron capture detector, $\mu \mathrm{ECD}$ ).

- Desarrollo de un método analítico para la determinación de clorofenoles en suelos mediante su derivatización y extracción in situ con anhídrido acético y la preconcentración con un sistema automático de MEPS acoplado a un cromatógrafo de gases con un detector de espectrometría de masas (mass spectrometer, MS). 
Determinación de compuestos de interés biológico en muestras de orina

- Desarrollo de un método analítico para la determinación de aminoácidos mediante su derivatización con cloroformiato de etilo acoplada a HS-PTV-GC-MS y aplicación a muestras de orina.

- Puesta a punto de un método analítico para la determinación en orina de posibles biomarcadores mediante HS-GC-MS.

La metodología seguida en el desarrollo de todos y cada uno de estos trabajos consiste en una serie de etapas fundamentales que se llevarán a cabo de manera secuencial:

\section{Optimización de la etapa de pretratamiento de muestra}

El suelo y la orina son matrices complejas, por lo que el pretratamiento de muestra empleado para la extracción de los analitos y la limpieza de las muestras es una etapa crucial en el desarrollo metodológico. Como ya se ha comentado en el apartado introductorio, esta Tesis aborda diversas técnicas de pretratamiento de muestra como QuEChERS, MEPS, reacciones de derivatización y HS, que se explicarán de forma más detallada en el siguiente apartado.

2. Optimización de las variables que afectan al inyector de temperatura programada, a la separación cromatográfica y a la detección

Todos los métodos analíticos que se proponen en esta Tesis están basados en la cromatografía de gases, por lo que la optimización de las variables involucradas en ella, así como las relacionadas con el sistema de 
inyección y detección, es una etapa muy importante para el adecuado desarrollo de un método analítico.

\section{Estudio del efecto de matriz}

Debido a la complejidad de las muestras de suelo y de orina, un aspecto fundamental que es necesario evaluar para determinar la aplicabilidad de los métodos propuestos a la determinación de los correspondientes analitos en dichas matrices es la existencia o no de efecto de matriz. De este estudio se desprenderá la posibilidad de utilizar calibración externa en el caso de que no exista efecto de matriz, o la necesidad de determinar los analitos en las muestras mediante el método de adición estándar en el caso de que el efecto de matriz esté presente.

\section{Determinación de las características analíticas del método propuesto}

En esta etapa se llevará a cabo la evaluación del comportamiento lineal de los calibrados, la utilización del análisis de varianza (analysis of variance, ANOVA) para comprobar el fallo de ajuste, la evaluación de la precisión del método a través de la repetibilidad y la reproducibilidad, y el cálculo de los límites de detección (limit of detection, LOD) y cuantificación (limit of quantification, LOQ).

\section{Validación del método propuesto}

Con el fin de comprobar que el procedimiento analítico propuesto es apto para las aplicaciones analíticas previstas es necesario validar el método. Para ello se utilizarán materiales de referencia certificados en los casos que 
sea posible o bien, muestras con concentraciones conocidas de los analitos de interés. 


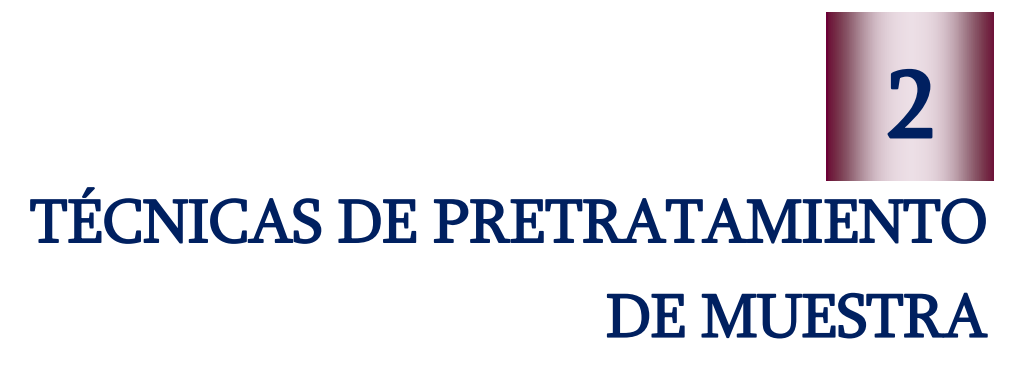





\subsection{QuEChERS}

La técnica de extracción líquido-líquido (liquid-liquid extraction, LLE) es una de las técnicas de preparación de muestra más consolidadas en los laboratorios de análisis. La versión clásica se ha utilizado durante muchos años y aún hoy sigue siendo uno de los métodos de extracción más empleados. Es un procedimiento sencillo y barato que no requiere equipamiento específico. Sin embargo, implica tiempos de análisis prolongados y utiliza grandes cantidades de disolventes orgánicos cuyo uso está cada vez más restringido, lo que ha dado lugar al desarrollo de versiones miniaturizadas del procedimiento convencional a las que se ha denominado técnicas de microextracción con disolventes (solvent microextraction, SME).

El objetivo de la LLE es la separación de compuestos basándose en las diferencias de solubilidades en dos líquidos inmiscibles. Generalmente, se ha aplicado para la extracción de compuestos orgánicos en agua o matrices con alto contenido de agua como frutas, verduras o fluidos biológicos. Para la extracción se utilizan disolventes orgánicos apolares (inmiscibles con el agua) de manera que los compuestos no polares, con mayor afinidad por la fase orgánica, se extraen en esta fase. El inconveniente de utilizar disolventes no polares es la limitada eficacia de la técnica para la extracción de compuestos orgánicos polares. Sin embargo, el uso de disolventes más polares, parcial o totalmente miscibles con el agua, no es posible al no conseguirse una adecuada separación de fases.

Para solucionar este inconveniente y poder ampliar la aplicación de la técnica LLE a la extracción de compuestos polares, se ha utilizado la adición de sales. El procedimiento consiste en añadir una alta concentración de sales 
sobre la mezcla de agua con un disolvente orgánico miscible o parcialmente miscible y, por la solvatación de los iones de las sales con las moléculas de agua, se consigue la separación de las dos fases miscibles (acuosa-orgánica) [1]. Este fenómeno se conoce como separación de fases inducida por sales y el procedimiento de extracción basado en este principio se denominó extracción líquido-líquido asistida por sales (salting-out assisted liquid-liquid extraction, SALLE) [2].

La principal desventaja de la técnica SALLE radica en que el extracto orgánico obtenido suele contener muchos compuestos polares co-extraídos de la matriz que, en muchas ocasiones, interfieren en la determinación de los compuestos objeto de estudio. Este hecho hace que, en la mayoría de los casos, sea necesario someter al extracto orgánico a diversas etapas de limpieza antes de llevar a cabo el procedimiento de determinación [2]. Otra posibilidad es la de analizar directamente el extracto, utilizando un detector altamente selectivo.

Las aplicaciones de esta técnica de extracción se han centrado especialmente en el área del análisis de pesticidas. El primer método multiresiduo (multiresidue method, MRM) que se desarrolló para el análisis de pesticidas en matrices alimenticias data de 1963 y es el denominado método Mills. La extracción de los compuestos se conseguía con acetonitrilo y, para lograr la separación de fases (acetonitrilo-agua) se utilizaron éter de petróleo y cloruro sódico [3].

También se desarrollaron métodos que utilizaban acetona como disolvente de extracción en lugar de acetonitrilo [4-6]. En todos ellos se utilizó 
un disolvente no polar (diclorometano o éter de petróleo) y cloruro sódico para lograr la separación de fases.

Los métodos citados, junto con las numerosas variaciones que se han desarrollado a partir de ellos, siguen utilizándose hoy día para el análisis de pesticidas en muchos laboratorios de todo el mundo.

A partir de los años 70, como consecuencia de los problemas para la salud humana y el medio ambiente relacionados con el uso de disolventes clorados, se desarrollaron nuevos métodos basados en SALLE en los que se conseguía la separación de fases simplemente añadiendo sales, es decir, sin necesidad de utilizar disolventes apolares para inducir dicha separación.

Algunas de las investigaciones se centraron en el sistema acetona-agua. Luke y colaboradores consiguieron la separación de ambas fases añadiendo una combinación de fructosa, sulfato de magnesio y cloruro sódico [7]. Otros autores lograron esta separación de fases utilizando diferentes combinaciones de sales $[8,9]$. Sin embargo, todos estos procedimientos presentan diversos inconvenientes relacionados con la alta miscibilidad de la acetona con el agua, por lo que no se logra una adecuada separación de fases sin la adición de disolventes apolares.

Otros estudios evaluaron el sistema acetonitrilo-agua. El acetonitrilo es menos miscible con el agua que la acetona, por lo que es posible lograr una separación de fases satisfactoria con la adición de la cantidad adecuada de sales. Lee y colaboradores utilizaron cloruro sódico para este propósito [10], y desde entonces se han publicado numerosos artículos que utilizan este método o 
pequeñas variaciones del mismo para la determinación de pesticidas en diferentes matrices [11-13].

Otro disolvente que se ha utilizado en el análisis de pesticidas es el acetato de etilo [14-16]. Éste, tiene la ventaja de ser lo suficientemente miscible con el agua como para lograr una adecuada penetración en los tejidos vegetales [17] y además su solubilidad en agua es menor que la de los otros disolventes citados, por lo que la separación de fases se consigue fácilmente con la adición de una sal desecante, capaz de eliminar el agua que queda en la fase orgánica $[18,19]$.

Durante los últimos 40 años también se han desarrollado, aunque en mucha menor proporción, algunos métodos basados en SALLE que se han aplicado a la determinación de compuestos que no son pesticidas. Matkovich y Christian ya aplicaron la separación inducida por sales del sistema acetonaagua en la extracción de quelatos metálicos [20]. Legget y colaboradores realizaron un estudio en el que utilizaban el sistema acetonitrilo-agua con separación de fases inducida por cloruro sódico para la determinación de concentraciones traza de explosivos en agua [21]. Además, la Agencia de Protección Medioambiental de Estados Unidos (United States Environmental Protection Agency, USEPA) ha propuesto un método (8330B) para la determinación de compuestos nitroaromáticos, nitroaminas y ésteres de nitrato, basado en la separación de fases acetonitrilo-agua inducida con cloruro sódico [22].

En el año 2003, Anastassiades y Lehotay [18] modificaron la técnica SALLE para adaptarla a las exigencias medioambientales y analíticas actuales. El nuevo método mantiene la simplicidad y eficacia del procedimiento 
convencional, pero utiliza la menor cantidad posible de muestra, así como una pequeña cantidad de disolventes orgánicos y material básico de laboratorio. La técnica se diseñó para la extracción de una amplia gama de pesticidas en matrices de frutas y verduras y se le asignó el nombre acrónimo de QuEChERS, que hace referencia a sus principales ventajas: rápido (quick), sencillo (easy), barato (cheap), eficaz (effective), robusto (rugged) y seguro (safe).

En el desarrollo del método se estudiaron diferentes disolventes de extracción (acetato de etilo, acetonitrilo y acetona), y con todos ellos se obtenían altas recuperaciones de los pesticidas de interés. Finalmente, se seleccionó el acetonitrilo debido a que presentaba mayor selectividad en la extracción de estos compuestos. Sin embargo, trabajando con otras matrices u otro tipo de compuestos cualquiera de los disolventes citados podría ser adecuado. También se estudiaron diferentes combinaciones de sales para lograr la separación de fases, seleccionando una mezcla de $\mathrm{NaCl}_{\text {y }} \mathrm{MgSO}_{4}$ (1:4) que era la más apropiada para lograr altas recuperaciones de los pesticidas más polares, al mismo tiempo que se evitaba la co-extracción de muchos compuestos polares de la matriz.

Además, los autores del método también simplificaron el procedimiento de limpieza que se empleaba tras la etapa de extracción. El nuevo procedimiento de limpieza se denominó extracción en fase sólida dispersiva (d-SPE). El proceso consiste en añadir un determinado volumen del extracto orgánico en un vial que contiene una pequeña cantidad de un material adsorbente; generalmente se ha utilizado amina primaria-secundaria (primary secondary amine, PSA). La mezcla se agita con un vórtex de manera que, idealmente, los compuestos interferentes de la matriz quedan retenidos en el 
adsorbente, mientras que los analitos objeto de estudio permanecen en el extracto. El siguiente paso es la eliminación del material adsorbente, mediante filtrado o centrifugación y, tras esta etapa, el extracto orgánico queda listo para su análisis. Este procedimiento ha demostrado ser altamente eficaz en la eliminación de gran parte de los compuestos lipídicos co-extraídos de la matriz. Además, es más rápido, más sencillo y requiere menos disolventes y materiales que el proceso de SPE convencional.

En la figura 2.1 se han representado de forma detallada las diferentes etapas del proceso completo (extracción + limpieza) desarrollado inicialmente para la extracción de pesticidas en matrices de frutas y verduras.

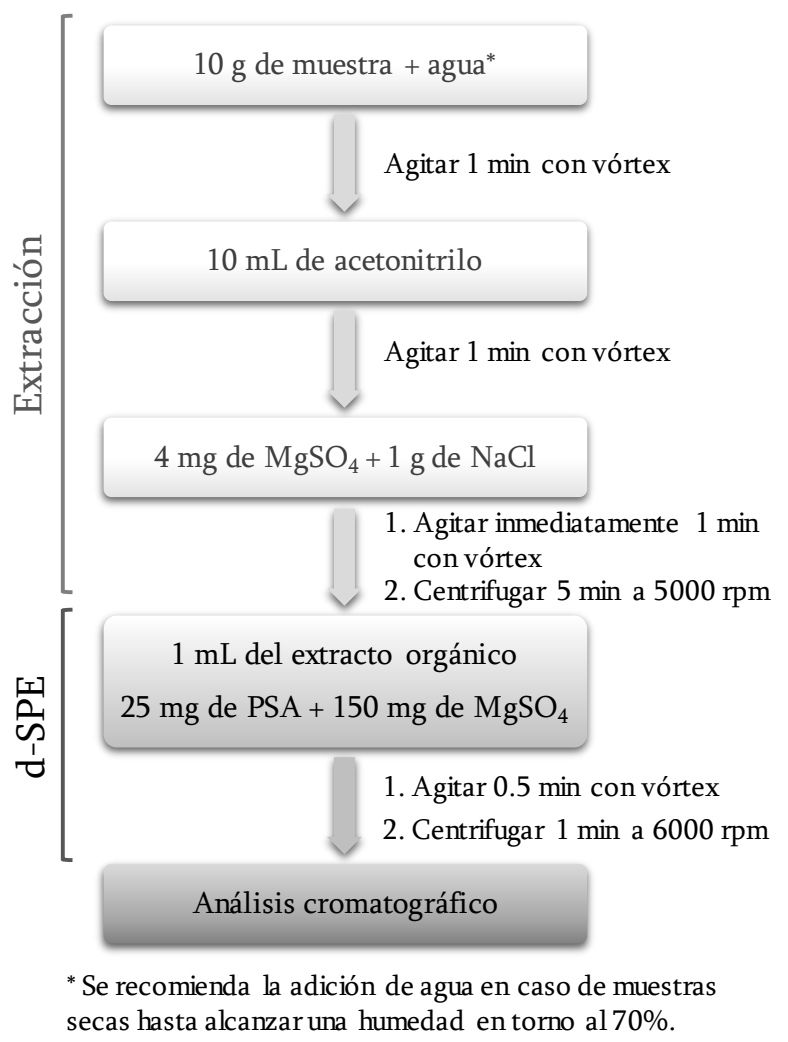

Figura 2.1. Etapas del procedimiento QuEChERS original 
A partir del procedimiento original, han surgido modificaciones relacionadas con la utilización de disoluciones tamponadas para mejorar la eficacia de la extracción de compuestos con propiedades ácido-base [23-25] y con la adición de agua sobre muestras secas, con el fin de obtener la humedad adecuada [26-28]. En la etapa de limpieza mediante d-SPE también se han desarrollado algunas modificaciones relacionadas con el uso de diferentes materiales adsorbentes. En el caso de matrices con alto contenido en grasas, se ha recomendado el uso de $\mathrm{C}_{18}$, junto con la PSA, ya que proporciona una limpieza adicional de los compuestos lipídicos co-extraídos [23,29]. Por otro lado, el uso de negro de carbón grafitizado (graphitized carbon black, GCB) ha demostrado su eficacia en la eliminación de clorofilas de los extractos [29]. También se han desarrollado algunas aplicaciones en las que se utiliza el procedimiento de limpieza mediante SPE clásica, en cartuchos [30-32].

El método ha recibido gran aceptación a nivel mundial y ha sido validado en estudios interlaboratorio para numerosos pesticidas en diversas matrices de alimentos [33-36]. Además, ha recibido la distinción de Método Oficial de la AOAC Internacional (Association of Analytical Communities) [37].

A pesar de que originalmente QuEChERS surgió como un método particular para el análisis de pesticidas en matrices alimentarias, que todavía hoy en día se sigue utilizando [38-41], su eficacia y flexibilidad lo han convertido en una potente herramienta para la determinación de otros tipos de compuestos y en otras matrices [42], tales como la determinación de compuestos farmacéuticos [43-47], hidrocarburos aromáticos policíclicos (polycyclic aromatic hydrocarbons, PAHs) [48-50], nitrosaminas [51] u 
hormonas esteroideas [52] en muestras de tipo biológico, o metilsiloxanos en productos para el cuidado personal [53].

Respecto a matrices de suelos, se ha utilizado para la determinación de pesticidas, insecticidas, fungicidas y herbicidas [54-58], fenoles y cresoles [59], compuestos farmacéuticos [60], así como compuestos aromáticos clorados [61] en diferentes tipos de suelos. Además, se ha propuesto una simplificación del método QuEChERS para la extracción de compuestos clorados en muestras de suelos [62] que elimina la etapa de limpieza debido a las características no grasas de esta matriz, y que se ha aplicado a la determinación de trihalometanos y BTEX (benceno, tolueno, etilbenceno y xileno) [63,64].

Esta versión simplificada de QuEChERS ha sido la base de partida para los diferentes estudios que se presentan en el desarrollo de un método analítico para la determinación de clorobencenos en suelos en el primer trabajo propuesto en esta tesis (I.1).

\section{Bibliografía}

[1] G.B. Frankforter, L. Cohen, Equilibria in the systems, water, acetone and inorganic salts, J. Am. Chem. Soc. 36 (1914) 1103-1134.

[2] R.E. Majors, Salting-out Liquid-Liquid Extraction (SALLE), LCGC North America, Jul 1 (2009).

[3] P.A. Mills, J.H. Onley, R.A. Gaither, Rapid method for chlorinated pesticide residues in nonfatty foods, J. Assoc. Off. Anal. Chem. 46 (1963) 186191. 
[4] G. Becker, Simultaneous gas chromatographic determination of chlorinated hydrocarbons and phosphates in plant material, Dtsch. Lebensm. Rundsch. 67 (1971) 125-126.

[5] M. Luke, J.E. Froberg, H.T. Masumoto, Extraction and cleanup of organochlorine, organophosphate, organonitrogen, and hydrocarbon pesticides in produce for determination by gas-liquid chromatography, J. Assoc. Off. Anal. Chem. 58 (1975) 1020-1026.

[6] W. Specht, M. Tilkes, Gas-chromatographische Bestimmung von Riickständen an Pflanzenbehandlungsmitteln nach Clean-up über GelChromatographie und Mini-Kieselgel-Säulen-Chromatographie, Fresenius J. Anal. Chem. 301 (1980) 300-307.

[7] M. Luke, I. Cassias, S. Yee, Lab. Inform. Bull. № 4178, Office of Regulatory Affairs, U.S. Food and Drugs Administration, 1999, Rockville, MD.

[8] F.J. Schenck, P. Callery, P.M. Gannett, J.R. Daft, S.J. Lehotay, Comparison of magnesium sulfate and sodium sulfate for removal of water from pesticide extracts of foods, J. AOAC Int. 85 (2002) 1177-1180.

[9] C.H. Parfitt, Lab. Inform. Bull. № 3616, Office of Regulatory Affairs, U.S. Food and Drug Administration, 1991, Rockville, MD.

[10] S.M. Lee, M.L. Papathakis, C.F. Hsiao-Ming, J.E. Carr, Multipesticide residue methods for fruits and vegetables: California Department of Food and Agriculture, Fresenius J. Anal. Chem. 339 (1991) 376-383.

[11] J. Fillion, R. Hindle, M. Lacroix, J. Selwyn, Multiresidue determination of pesticides in fruit and vegetables by gas chromatography-mass-selective 
detection and liquid chromatography with fluorescence detection, J. AOAC Int. 78 (1995) 1352-1366.

[12] R.S. Sheridan, J.R. Meola, Analysis of pesticide residues in fruits, vegetables and milk by gas chromatography/tandem mass spectrometry, J. AOAC Int. 82 (1999) 982-990.

[13] S.J. Leothay, A.R. Lightfield, J.A. Harman-Fetcho, D.A. Donoghue, Analysis of Pesticide Residues in eggs by direct sample Introduction/Gas chromatography/Tandem Mass Spectrometry, J. Agric. Food Chem. 49 (2001) 4589-4596.

[14] A. Anderson, H. Palsheden, Comparison of the efficiency of different GLC multi-residue methods on crops containing pesticide residues, Fresenius J. Anal. Chem. 339 (1991) 365-367.

[15] A.R. Fernández-Alba, A. Valverde, A. Agüera, M. Contreras, Gas chromatographic determination of organochlorine and pyrethroid pesticides of horticultural concern, J. Chromatogr. A 686 (1994) 263-274.

[16] K. Barnerjee, D.P. Oulkar, S. Dasgupta, S.B. Patil, S.H. Patil, R. Savant, P.G. Adsule, Validation and uncertainty analysis of a multi-residue method for pesticides in graps using ethyl acetate extraction and liquid chromatography-tandem mass spectrometry, J. Chromatogr. A 1173 (2007) 98-109.

[17] A. Hercegová, M. Dömötörová, E. Matisová, Sample preparation methods in the analysis of pesticide residues in baby food with subsequent chromatographic determination, J. Chromatogr. A. 1153 (2007) 54-73. 
[18] M. Anastassiades, S.J. Lehotay, D. Stajnbaher, F.J. Schenck, Fast and easy multiresidue method employing acetonitrile extraction/partitioning and "dispersive solid-phase extraction" for the determination of pesticide residues in produce, J. AOAC Int. 86 (2003) 412-431.

[19] K. Mastovská, S.J. Lehotay, Evaluation of common organic solvents for gas chromatographic analysis and stability of multiclass pesticide residues, J. Chromatogr. A 1040 (2004) 259-272.

[20] C.E. Matkovich, G.D. Christian, Salting-out of acetone from water-basis of a new solvent extraction system, Anal. Chem. 45 (1973) 1915-1920.

[21] D.C. Leggett, R.F. Jenkis, P.H. Miyares, Salting-out solvent extraction for preconcentration of neutral polar organic solutes from water, Anal. Chem. 62 (1990) 1355-1356.

[22] USEPA Method 8330B (SW-846): nitroaromatics, nitramines and nitrate esters by high performance liquid chromatography (HPLC). Revision 2 USEPA, Washington, USA, 2006.

https://www.epa.gov/sites/production/files/2015-07/documents/epa-8330b.pdf. Fecha de acceso: 8 de marzo de 2017.

[23] S.J. Lehotay, K. Matovská, S.J. Yun, Evaluation of two fast and easy methods for pesticide residue analysis in fatty food matrixes, J. AOAC Int. 88 (2005) 630-638.

[24] S.J. Lehotay, K.A. Son, H. Kwon, U. Koesukwiwat, W. Fu, K. Mastovská, E. Hoh, N. Leepipatpiboon, Comparison of QuEChERS sample preparation methods for the analysis of pesticide residues in fruits and vegetables, J. Chromatogr. A 1217 (2010) 2548-2560. 
[25] S.J. Lehotay, K. Mastovská, A.R. Lightfield, Use of buffering and other means to improve results of problematic pesticides in a fast and easy method for residue analysis of fruits and vegetables, J. AOAC Int. 88 (2005) 615-629.

[26] C. Díez, W.A. Traag, P. Zommer, P. Marinero, J. Atienza, Comparison of an acetonitrile extraction/partitioning and "dispersive solid-phase microextraction" method with classical multi-residue methods for the extraction of herbicide residues in barley samples, J. Chromatogr. A 1131 (2006) 11-23.

[27] T.D. Nguyen, E.M. Han, M.S. Seo, S.R. Kim, M.Y. Yun, D.M. Lee, G.H. Lee, A multi-residue method for the determination of 203 pesticides in rice paddies using gas chromatography/mass spectrometry, Anal. Chim. Acta 619 (2008) 67-74.

[28] P. Payá, M. Anastassiades, D. Mack, I. Sigalova, B. Tasdelen, J. Oliva, A. Barba, Analysis of pesticide residues using the Quick Easy Cheap Effective Rugged and Safe (QuEChERS) pesticide multiresidue method in combination with gas and liquid chromatography and tandem mass spectrometric detection, Anal. Bioanal. Chem. 389 (2007) 1697-1714.

[29] www.quechers.com. Fecha de acceso: 8 de marzo de 2017.

[30] F.J. Schenck, A.N. Brown, L.V. Podhorniak, A. Parker, M. Reliford, J.W. Wong, A rapid multiresidue method for determination of pesticides in fruits and vegetables by using acetonitrile extraction/partitioning and solid phase extraction column cleanup, J. AOAC Int. 91 (2008) 422-438.

[31] M.A. Aramendia, V. Borau, F. Lafont, A. Marinas, J.M. Marinas, J.M. Moreno, F.J. Urbano, Determination of herbicides residues in olive soil by gas 
chromatography-tandem mass spectrometry, Food Chem. 105 (2007) 855-861. [32] A. Garrido Frenich, J.L. Martínez Vidal, E. Pastor-Montoro, R. RomeroGonzález, High-throughput determination of pesticide residues in food commodities by use of ultra-performance liquid chromatography-tandem mass spectrometry, Anal. Bioanal. Chem. 390 (2008) 947-959.

[33] F.J. Schenck, J.E. Hobbs, Evaluation of the Quick, Easy, Cheap, Effective, Rugged, and Safe (QuEChERS) approach to pesticide residue analysis, Bull. Environ. Contam. Toxicol. 73 (2004) 24-30.

[34] C. Lesueur, P. Knittl, M. Gartner, A. Mentler, M. Fuerhacker, Analysis of 140 pesticides from conventional farming foodstuff samples after extraction with the modified QuEChERS method, Food Control 19 (2008) 906-914.

[35] E. Ciéslik, A. Sadowska-Rociek, J.M.M. Ruíz, M. Surma-Zadora, Evaluation of QuEChERS method for the determination of organochlorine pesticide residues in selected groups of fruits, Food Chem. 125 (2011) 773-778.

[36] C. Sack, M. Smoker, N. Chamkasem, R. Thompson, G. Satterfield, C. Masse, G. Mercer, B. Neuhaus, I. Cassias, E. Chang, Y. Lin, S. MacMahon, J. Wong, K. Zhang, R.E. Smith, Collaborative Validation of the QuEChERS procedure for the determination of pesticides in food by LC-MS/MS, J. Agric. Food Chem. 59 (2011) 6383-6411.

[37] S.J. Lehotay, Determination of pesticide residues in foods by acetonitrile extraction and partitioning with magnesium sulfate: collaborative study, J. AOAC Int. 90 (2007) 485-520.

[38] A. Wilkowska, M. Biziuk, Determination of pesticides residues in food 
matrices using the QuEChERS methodology, Food Chem. 125 (2011) 803-812. [39] P. Kaewsuya, W.E. Brewer, J. Wong, S.L. Morgan, Automated QuEChERS tips for analysis of pesticide residues in fruits and vegetables by GC-MS, J. Agric. Food Chem. 61 (2013) 2299-2314.

[40] Y. Chen, S. Lopez, D.G. Hayward, H.Y. Park, J.W. Wong, S.S. Kim, J. Wan, R.M. Reddy, D.J. Quinn, D. Steiniger, Determination of multiresidues pesticides in botanical dietary supplements using gas chromatography-triple quadrupole mass spectrometry (GC-MS/MS), J. Agric. Food Chem. 64 (2016) 6125-6132.

[41] X-C. Wang, B. Shu, S. Li, Z-G. Yang, B. Qiu, QuEChERS followed by dispersive liquid-liquid microextraction based on solidification of floating organic droplet method for organochlorine pesticides analysis in fish, Talanta 162 (2017) 90-97.

[42] M.Á. González Curbelo, B. Socas Rodríguez, A.V. Herrera Herrera, J. González Sálamo, J. Hernández Borges, M.Á. Rodríguez Delgado, Evolution and applications of the QuEChERS method, Trends Anal. Chem. 71 (2015) 169-185.

[43] F. Plössl, M. Giera, F. Bracher, Multiresidue analytical method using dispersive solid-phase extraction and gas chromatography/ion trap mass spectrometry to determine pharmaceuticals in whole blood, J. Chromatogr. A 1135 (2006) 19-26.

[44] M.M. Aguilera Luiz, J.L. Martínez Vidal, R. Romero González, A. Garrido Frenich, Multi-residue determination of veterinary drugs in milk by ultra-high-pressure liquid chromatography-tandem mass spectrometry, J. 
Chromatogr. A 1205 (2008) 10-16.

[45] G. Stubbings, T. Bigwood, The development and validation of a multiclass liquid chromatography tandem mass spectrometry (LC-MS/MS) procedure for the determination of veterinary drug residues in animal tissue using a QuEChERS (Quick, Easy, Cheap, Effective, Rugged and Safe) approach, Anal. Chim. Acta 637 (2009) 68-78.

[46] C-H. Wen, S-L. Lin, M-R. Fuh, Determination of sulfonamides in animal tissues by modified QuEChERS and liquid chromatography tandem mass spectrometry, Talanta 164 (2017) 85-91.

[47] P. Mu, N. Xu, T. Chai, Q. Jia, Z. Yin, S. Yang, Y. Qian, J. Qiu, Simultaneous determination of 14 antiviral drugs and relevant metabolites in chicken muscle by UPLC-MS/MS after QuEChERS preparation, J. Chromatogr. B 1023-1024 (2016) 17-23.

[48] M.J. Ramalhosa, P. Paíga, S. Morais, C. Delerue-Matos, M.B.P.P. Oliveira, Analysis of polycyclic aromatic hydrocarbons in fish: evaluation of a quick, easy, cheap, effective, rugged and safe extraction method, J. Sep. Sci. 32 (2009) 3529-3538.

[49] T.H. Kao, S. Chen, C.J. Chen, C.W. Huang, B.H. Chen, Evaluation of analysis of polycyclic aromatic hydrocarbons by the QuEChERS method and gas chromatography-mass spectrometry and their formation in poultry meat as affected by marinating and frying, J. Agric. Food Chem. 60 (2012) 13801389.

[50] M. Surma, A. Sadowska-Rociek, E. Cieślik, The application of d-SPE in the QuEChERS method for the determination of PAHs in food of animal 
origin with GC-MS detection, Eur. Food Res. Technol. 238 (2014) 1029-1036.

[51] S.J. Lehotay, Y. Sapozhnikova, L. Han, J.J. Johnston, Analysis of nitrosamines in cooked bacon by QuEChERS simple preparation and gas chromatography-tandem mass spectrometry with backflushing, J. Agric. Food Chem. 63 (2015) 10341-10351.

[52] X-T. Tan, Z-M. Li, L-G. Deng, S-C. Zhao, M-I. Wang, Analysis of 13 kinds of steroid hormones in raw milk using modified QuEChERS method combined with UPLC-QTOF-MS, J. Integr. Agr. 15 (2016) 2163-2174.

[53] D. Capela, V. Homem, A. Alves, L. Santos, Volatile methylsiloxanes in personal care products - using QuEChERS as a "Green" analytical approach, Talanta 155 (2016) 94-100.

[54] D. Drożdżyński, J. Kowalska, Rapid analysis of organic farming insecticides in soil and produce using ultra-performance liquid chromatography/tandem mass spectrometry, Anal. Bioanal. Chem. 394 (2009) 2241-2247.

[55] M. Asensio Ramos, J. Hernández Borges, L.M. Ravelo Pérez, M.A. Rodríguez Delgado, Evaluation of a modified QuEChERS method for the extraction of pesticides from agricultural, ornamental and forestal soils, Anal. Bioanal. Chem. 396 (2010) 2307-2319.

[56] S.S. Caldas, C.M. Bolzan, M.B. Cerqueira, D. Tomasini, E.B. Furlong, C. Fagundes, E.G. Primel, Evaluation of a modified QuEChERS extraction of multiple classes of pesticides from a rice paddy soil by LC-APCI-MS/MS, J. Agric. Food Chem. 59 (2011) 11918-11926. 
[57] Y. Cheng, F. Dong, X. Liu, J. Xu, W. Meng, N. Liu, Z. Chen, Y. Tao, Y. Zheng, Simultaneous determination of fipronil and its major metabolites in corn and soil by ultra-performance liquid chromatography-tandem mass spectrometry, Anal. Methods 6 (2014) 1788-1795.

[58] P. Kaczyński, B. Łozowicka, M. Jankowska, I. Hrynko, Rapid determination of acid herbicides in soil by liquid chromatography with tandem mass spectrometric detection based on dispersive solid phase extraction, Talanta 152 (2016) 127-136.

[59] J.A. Padilla Sánchez, P. Plaza Bolaños, R. Romero González, A. Garrido Frenich, J.L. Martínez Vidal, Application of a quick, easy, cheap, effective, rugged and safe-based method for the simultaneous extraction of chlorophenols, alkylphenols, nitrophenols and cresols in agricultural soils, analyzed by using gas chromatography-triple quadrupole-mass spectrometry/mass spectrometry, J. Chromatogr. A 1217 (2010) 5724-5731.

[60] M-V. Salvia, E. Vulliet, L. Wiest, R. Baudot, C. Cren-Olivé, Development of a multi-residue method using acetonitrile-based extraction followed by liquid chromatography-tandem mass spectrometry for the analysis of steroids and veterinary and human drugs at trace levels in soil, J. Chromatogr. A 1245 (2012) 122-133.

[61] F. Rouvière, A. Buleté, C. Cren-Olivé, C. Arnaudguilhem, Multiresidue analysis of aromatic organochlorines in soil by gas chromatography-mass spectrometry and QuEChERS extraction based on water/dichloromethane partitioning. Comparison with accelerated solvent extraction, Talanta 93 (2012) 336-344. 
[62] C. García Pinto, M.E. Fernández Laespada, S. Herrero Martín, A.M. Casas Ferreira, J.L. Pérez Pavón, B. Moreno Cordero, Simplified QuEChERS approach for the extraction of chlorinated compounds from soil samples, Talanta 81 (2010) 385-391.

[63] S. Herrero Martín, C. García Pinto, J.L. Pérez Pavón, B. Moreno Cordero, Determination of trihalomethanes in soil matrices by simplified quick, easy, cheap, effective, rugged and safe extraction and fast gas chromatography with electron capture detection, J. Chromatogr. A 1217 (2010) 4883-4889.

[64] C. García Pinto, S. Herrero Martín, J.L. Pérez Pavón, B. Moreno Cordero, A simplified Quick, Easy, Cheap, Effective, Rugged, and Safe approach for the determination of trihalomethanes and benzene, toluene, ethylbenzene, and xylenes in soil matrices by fast gas chromatography with mass spectrometry detection, Anal. Chim. Acta 689 (2011) 129-136. 


\subsection{Microextracción con sorbentes empaquetados}

La microextracción con sorbentes empaquetados (MEPS) es una técnica de preparación de muestra basada en la miniaturización y automatización del proceso de extracción en fase sólida convencional (SPE). Esta técnica fue desarrollada y patentada por el grupo de Mohamed AbdelRehim en 2004 [1,2].

Se trata de una técnica de preparación de muestras líquidas y tiene como objetivo la extracción de compuestos de interés contenidos en las mismas. Esta técnica requiere la utilización de un dispositivo consistente en una jeringa provista de un cartucho que contiene una cierta cantidad de sorbente (entre 0.5 y $4 \mathrm{mg}$ ). El cartucho se sitúa en el extremo de la aguja que conecta con el tubo de la jeringa. De esta manera, las muestras aspiradas a través de la jeringa tienen que atravesar el cartucho que contiene el sorbente y en el que quedan retenidos los compuestos de interés. La aguja se fija a la jeringa con una tuerca de cierre, como se puede observar en la figura 2.2.

Los materiales más comunes utilizados como sorbentes son sílice, $\mathrm{C}_{18}$, $\mathrm{C}_{8}, \mathrm{C}_{2}$ (estos tres con una base de sílice) o intercambiadores fuertes de cationes, aunque también pueden utilizarse materiales de acceso restringido (restricted access materials, RAMs) o materiales poliméricos como los polímeros de impresión molecular (molecularly imprinted polymers, MIPs) [3]. Para conseguir una preconcentración de los analitos de interés es necesario seleccionar el sorbente adecuado de acuerdo a las características de los mismos. Tanto el tamaño del cartucho como el de las partículas del material sorbente deben ser lo más pequeños posible para aumentar la velocidad de transferencia de los analitos desde la muestra líquida al sorbente del cartucho. 


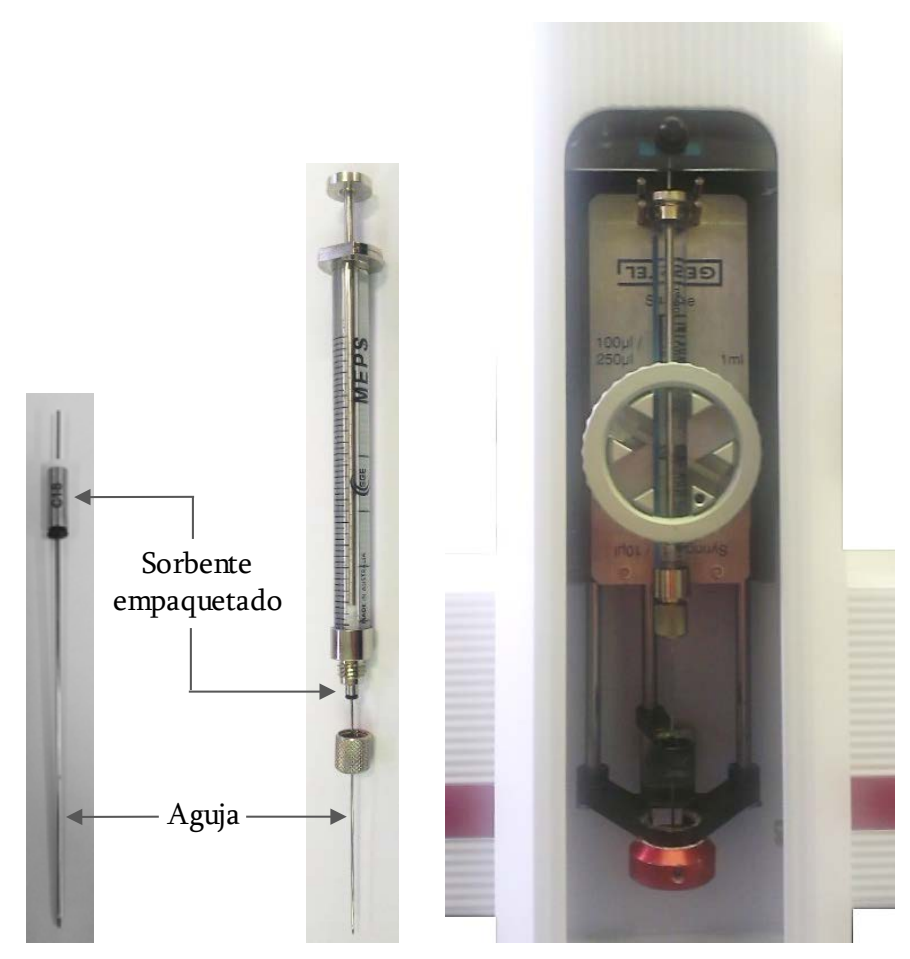

Figura 2.2. Cartucho $C_{18}$, jeringa de MEPS y disposición de la jeringa en el sistema de inyección automática

La separación se realiza mediante una serie de aspiraciones y expulsiones consecutivas de la muestra líquida a través del cartucho previamente acondicionado, consiguiéndose la retención de los analitos en el sorbente empaquetado. El cartucho se lava entonces con agua ultrapura u otra disolución de lavado para eliminar interferencias y, a continuación, se lleva a cabo una etapa de secado. Posteriormente, la elución de los analitos se efectúa con una pequeña cantidad de disolvente orgánico que se hace pasar a través de la jeringa y puede ser inyectada directamente en un sistema de análisis, si el instrumento está automatizado, o depositada en un vial de muestreo en caso de tratarse de un dispositivo manual. El esquema del proceso se muestra en la 
figura 2.3.

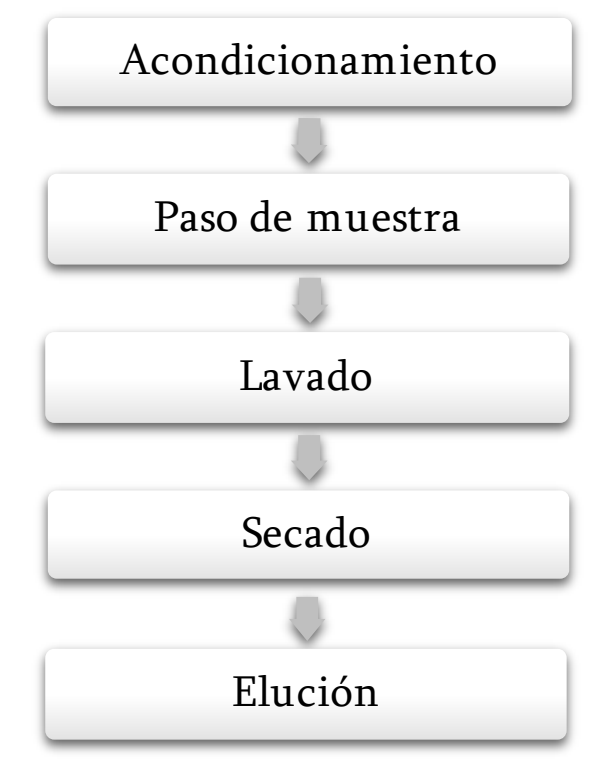

Figura 2.3. Esquema de un proceso de extracción mediante MEPS

Existen dos modalidades de trabajo en MEPS que se diferencian en la etapa de extracción de la muestra [4]. En una de ellas, la muestra se pasa por el cartucho una vez y se desecha, repitiéndose esta operación con diferentes alícuotas de muestra hasta coger el volumen total. Esta modalidad se conoce con el nombre de extract-discard. En la otra modalidad existente, multiple $d r a w$-eject, un volumen fijo de muestra se deposita en un vial y se realizan múltiples tomas y eluciones de la misma siempre del mismo vial. Esta segunda modalidad es muy interesante cuando el objetivo es extraer la mayor cantidad posible de analitos de volúmenes muy pequeños de muestra, siendo muy útil su aplicación en bioanálisis [5-7].

Ambas modalidades de microextracción con sorbentes empaquetados se pueden realizar mediante dos formas de trabajo diferentes: manual y 
automática [8]. En la primera, el desplazamiento del émbolo es automático, pero las diferentes etapas del proceso de extracción se llevan a cabo de forma manual. El volumen total de eluyente se lleva a un vial y desde ahí una parte se inyecta en el sistema de análisis. En la segunda, la jeringa MEPS con el cartucho forma parte de un sistema de inyección automática acoplado en línea con el sistema de análisis (figura 2.2), por lo que tanto el desplazamiento del émbolo como las diferentes etapas del proceso se llevan a cabo de forma automática y todo el eluyente es inyectado en el sistema de análisis. Los cartuchos son los mismos para ambas formas de trabajo, existiendo diferentes tipos de puntas y grosores de aguja en función de si el acoplamiento del MEPS automático es a un cromatógrafo de gases o de líquidos.

El procedimiento manual ofrece la posibilidad de tomar la muestra in situ [9] y posteriormente, en el laboratorio, realizar la elución de los analitos. Sin embargo, la principal ventaja de la forma automática de trabajo es que todo el extracto se inyecta en el cromatógrafo, mientras que en la manual sólo se inyecta una pequeña parte. Además, trabajando de forma automática los volúmenes que se utilizan para eluir los compuestos son mucho más pequeños que los permitidos con el émbolo digital de la modalidad manual.

Esta técnica MEPS surge a partir de los intentos de agilizar y hacer más eficiente la extracción en fase sólida. En especial, el desarrollo de técnicas más rápidas de tratamiento de muestra resulta interesante debido a que la mayor parte del tiempo total de análisis se invierte en los procesos previos a la propia identificación y cuantificación de los compuestos de interés. La extracción en fase sólida (SPE) así como la microextracción en fase sólida (solid-phase microextraction, SPME) reemplazaron en poco tiempo a otras técnicas de 
extracción que consumían mayor cantidad de disolventes como los procesos de extracción líquido-líquido (LLE). La microextracción con sorbentes empaquetados (MEPS) pretende mejorar algunos aspectos de estas dos primeras metodologías.

El principio en el que se basa la microextracción con sorbentes empaquetados, el mismo que SPE, es la diferente interacción de los analitos entre el sorbente y la muestra líquida. En este caso, la elución de los analitos retenidos se realiza mediante unos pocos microlitros de un disolvente orgánico como, por ejemplo, metanol, diclorometano o acetato de etilo. Frente a la extracción en fase sólida convencional, la metodología MEPS reduce significativamente el tiempo necesario para preparar e inyectar las muestras, puede combinarse con inyectores automáticos de cromatografía de líquidos y de gases (la etapa de extracción e inyección se efectúan en línea usando la misma jeringa) y se puede trabajar en un margen de volúmenes de muestra desde unos pocos microlitros hasta varios mililitros. Al igual que en SPE, con MEPS se consigue una extracción de los compuestos de interés presentes en la muestra, pero además, se consigue una preconcentración de los mismos y se utiliza, generalmente, una cantidad menor de disolvente orgánico para su elución [9].

Además, la pequeña cantidad de sorbente que se encuentra dentro del cartucho de MEPS puede limpiarse de una manera fácil y efectiva entre muestras utilizando pequeños volúmenes de disolventes para reducir el riesgo de contaminación entre ellas (efecto memoria). Los cartuchos de MEPS pueden reutilizarse un gran número de veces, siempre que se optimicen las condiciones de limpieza [5]. Es por todo esto que esta nueva técnica es más 
ecológica y económica que muchos otros métodos de preconcentración. De acuerdo con la Química Analítica “verde” [10,11], utiliza pequeñas cantidades de productos químicos peligrosos y reduce los costes energéticos y los desechos.

En cuanto a las aplicaciones de esta técnica, la microextracción con sorbentes empaquetados se ha utilizado principalmente en bioanálisis $[3,12,13]$ en matrices tales como plasma humano, orina, sangre o saliva, siendo muy importante para este tipo de muestras la función de MEPS de eliminar los componentes interferentes de la matriz y de aislar y concentrar selectivamente los analitos de interés $[5,14,15]$. Se ha utilizado para el análisis en plasma de diferentes fármacos como anestésicos locales [1], antidepresivos [5], $\beta$ bloqueadores usados para tratar trastornos del ritmo cardíaco [16], antipsicóticos [6,17,18], antiepilépticos [19-21] y antiinflamatorios no esteroideos [22], así como compuestos opioides [14], cannabinoides [23] y aminas biogénicas [7].

Se ha extendido el uso de esta técnica para explorar el perfil metabólico de la orina [24] y se han propuesto aplicaciones para la detección en esta matriz de compuestos endógenos como la carnitina y las acilcarnitinas [15], poliaminas [25], leucotrieno $\mathrm{B}_{4}$ [26], y catecolaminas [27], estas últimas tanto en orina como en plasma utilizando métodos innovadores de recogida de muestra como son las manchas de plasma y orina secas, más conocidos por sus nombres en inglés: dried plasma spots (DPSs) y dried urine spots (DUSs). En orina, además de compuestos endógenos, se ha utilizado la técnica MEPS para el análisis de antipsicóticos [17], antiepilépticos [19,20], antidepresivos [28], antiinflamatorios no esteroideos [22], parabenos [29] y drogas como la 
ketamina y sus metabolitos mayoritarios [30], estos últimos también medidos en plasma.

La orina y el plasma son las matrices más utilizadas en bioanálisis. En menor medida también se han propuesto metodologías en las que se incluye una etapa de microextracción con sorbentes empaquetados para el análisis de saliva $[17,21,31,32]$, esputos [33] y muestras de sangre [34,35]. Para análisis en sangre también se ha utilizado el método de recogida de muestra de manchas de sangre secas (dried blood spots, DBSs) [36].

Los estudios en el campo del análisis de alimentos se centran principalmente en el análisis de bebidas como cerveza [37], grappa [38], vino [39-44] y bebidas sin alcohol [45-47]. Existe también alguna aplicación en otros alimentos como huevo [48], miel [49] y carne de cerdo [50].

Además, la técnica MEPS se ha aplicado para el estudio de otras matrices como, por ejemplo, para el análisis de ftalatos en cosméticos [46] y de aminas aromáticas provenientes de tintes azoicos de textiles [51].

También se ha utilizado esta técnica para el análisis de muestras medioambientales, principalmente de aguas. En este caso las aplicaciones incluyen: contaminantes orgánicos prioritarios [52], compuestos policíclicos [2,53], aminas aromáticas [54], clorobencenos [55], ácidos haloacéticos [56], disruptores endocrinos como parabenos, triclosán y metil triclosán [57], fluoroquinolonas [58] y medicamentos antiinflamatorios no esteroideos [59]. Sin embargo, su aplicación a otras matrices medioambientales se reduce al estudio de bromodifeniléteres, utilizados como retardantes de llama, en lodos de depuradora [60] y algunos pesticidas en suelos [61]. 
Por ello, la aplicación de MEPS que se propone en esta tesis (I.2) para la determinación de clorofenoles en muestras de suelos después de su derivatización y extracción in situ resulta ser, no sólo una metodología innovadora para el estudio de estos compuestos en suelos, sino también una metodología rápida y simple al utilizar la modalidad automática de MEPS mediante el automuestrador MPS2 de Gerstel (Multi Purpose Sampler, Alemania) que acopla en línea esta técnica con la cromatografía de gases.

\section{Bibliografía}

[1] M. Abdel-Rehim, New trend in simple preparation: on-line microextraction in packed syringe for liquid and gas chromatography applications. I. Determination of local anaesthetics in human plasma samples using gas chromatography-mass spectrometry, J. Chromatogr. B 801 (2004) 317-321.

[2] A. El-Beqqali, A. Kussak, M. Abdel-Rehim, Fast and sensitive environmental analysis utilizing microextraction in packed syringe online with gas chromatography-mass spectrometry. Determination of polycyclic aromatic hydrocarbons in water, J. Chromatogr. A 1114 (2006) 234-238.

[3] L. Yang, R. Said, M. Abdel-Rehim, Sorbent, device, matrix application in microextraction by packed sorbent (MEPS): A review, J. Chromatogr. B 1043 (2017) 33-43.

[4] M. Quinto, G. Spadaccino, D. Nardiello, C. Palermo, P. Amodio, D. Li, 
D. Centonze, Microextraction by packed sorbent coupled with gas chromatography-mass spectrometry: A comparison between "draw-eject" and "extract-discard" methods under equilibrium conditions for the determination of polycyclic aromatic hydrocarbons, J. Chromatogr. A 1371 (2014) 30-38.

[5] A.R. Chaves, F.Z. Leandro, J.A. Carris, M.E. C. Queiroz, Microextraction in packed sorbent for analysis of antidepressants in human plasma by liquid chromatography and spectrophotometric detection, J. Chromatogr. B 878 (2010) 2123-2129.

[6] I.D. de Souza, D.S. Domingues, M.E.C. Queiroz, Hybrid silica monolith for microextraction by packed sorbent to determine drugs from plasma samples by liquid chromatography-tandem mass spectrometry, Talanta 140 (2015) 166-175.

[7] L. Konieczna, A. Roszkowska, A. Synakiewicz, T. Stachowicz-Stencel, E. Adamkiewicz-Drożyńska, T. Bączek, Analytical approach to determining human biogenic amines and their metabolites using eVol microextraction in packed syringe coupled to liquid chromatography mass spectrometry method with hydrophilic interaction chromatography column, Talanta 150 (2016) 331-339.

[8] M.M. Moein, A. Abdel-Rehim, M. Abdel-Rehim, Microextraction by packed sorbent (MEPS), TrAC-Trend. Anal. Chem. 67 (2015) 34-44.

[9] C. Duan, Z. Shen, D. Wu, Y. Guan, Recent developments in solid-phase microextraction for on-site sampling and sample preparation, TrAC-Trend. Anal. Chem. 30 (2011) 1568-1574.

[10] M. Farré, S. Pérez, C. Gonçalves, M.F. Alpendurada, D. Barceló, Green 
analytical chemistry in the determination of organic pollutants in the aquatic environment, TrAC-Trend. Anal. Chem. 29 (2010) 1347-1362.

[11] A. Páleníková, S. Hrouzková, Microextraction in packed syringe: solvent-minimized simple preparation technique, Monatsh. Chem. 145 (2014) 537-549.

[12] M. Abdel-Rehim, Recent advances in microextraction by packed sorbent for bioanalysis, J. Chromatogr. A 1217 (2010) 2569-2580.

[13] L. Nováková, H. Vlčková, A review of current trends and advances in modern bio-analytical methods: Chromatography and simple preparation, Anal. Chim. Acta 656 (2009) 8-35.

[14] L. Somaini, M.A. Saracino, C. Marcheselli, S. Zanchini, G. Gerra, M.A. Raggi, Combined liquid chromatography-coulometric detection and microextraction by packed sorbent for the plasma analysis of long acting opioids in heroin addicted patients, Anal. Chim. Acta 702 (2011) 280-287.

[15] S. Magiera, J. Baranowski, Determination of carnitine and acylcarnitines in human urine by means of microextraction in packed sorbent and hydrophilic interaction chromatography-ultra-high-performance liquid chromatography-tandem mass spectrometry, J. Pharmaceut. Biomed. 109 (2015) 171-176.

[16] T. Abuzooda, A. Amini, M. Abdel-Rehim, Graphite-based microextraction by packed sorbent for online extraction of $\beta$-blockers from human plasma samples, J. Chromatogr. B 992 (2015) 86-90.

[17] R. Mandrioli, L. Mercolini, D. Lateana, G. Boncompagni, M.A. Raggi, 
Analysis of risperidone and 9-hydroxyrisperidone in human plasma, urine and saliva by MEPS-LC-UV, J. Chromatogr. B 879 (2011) 167-173.

[18] B.M. Fonseca, I.E.D. Moreno, M. Barroso, S. Costa, J.A. Queiroz, E. Gallardo, Determination of seven selected antipsychotic drugs in human plasma using microextraction in packed sorbent and gas chromatographytandem mass spectrometry, Anal. Bioanal. Chem. 405 (2013) 3953-3963.

[19] S. Rani, A.K. Malik, B. Singh, Novel micro-extraction by packed sorbent procedure for the liquid chromatographic analysis of antiepileptic drugs in human plasma and urine, J. Sep. Sci. 35 (2012) 359-366.

[20] S. Rani, A.K. Malik, A novel microextraction by packed sorbent-gas chromatography procedure for the simultaneous analysis of antiepileptic drugs in human plasma and urine, J. Sep. Sci. 35 (2012) 2970-2977.

[21] S. Ventura, M. Rodrigues, S. Pousinho, A. Falcão, G. Alves, Determination of lamotrigine in human plasma and saliva using microextraction by packed sorbent and high performance liquid chromatography-diode array detection: An innovative bioanalytical tool for therapeutic drug monitoring, Microchem J. 30 (2017) 221-228.

[22] M. Locatelli, V. Ferrone, R. Cifelli, R.C. Barbacane, G. Carlucci, Microextraction by packed sorbent and high performance liquid chromatography determination of seven non-steroidal anti-inflammatory drugs in human plasma and urine, J. Chromatogr. A 1367 (2014) 1-8.

[23] T. Rosado, L. Fernandes, M. Barroso, E. Gallardo, Sensitive determination of THC and main metabolites in human plasma by means of microextraction by packed sorbent and gas chromatography-tandem mass 
spectrometry, J. Chromatogr. B 1043 (2017) 63-73.

[24] C. Silva, C. Cavaco, R. Perestrelo, J. Pereira, J.S. Câmara, Microextraction by packed sorbent (MEPS) and Solid-Phase microextraction (SPME) as sample preparation procedures for the metabolomic profiling of urine, Metabolites 4 (2014) 71-97.

[25] A.M. Casas Ferreira, B. Moreno Cordero, Á.P. Crisolino Pozas, J.L. Pérez Pavón, Use of microextraction by packed sorbents and gas chromatographymass spectrometry for the determination of polyamines and related compounds in urine, J. Chromatogr. A 1444 (2016) 32-41.

[26] R. Perestrelo, C.L. Silva, J.S. Câmara, Determination of urinary levels of leukotriene $\mathrm{B}_{4}$ using a highly specific and sensitive methodology based on automatic MEPS combined with UHPLC-PDA analysis, Talanta 144 (2015) 382-389.

[27] M.A. Saracino, L. Santarcangelo, M.A. Raggi, L. Mercolini, Microextraction by packed sorbent (MEPS) to analyze catecholamines in innovative biological samples, J. Pharmaceut. Biomed. 104 (2015) 122-129.

[28] V. Alves, J. Gonçalves, C. Conceição, H.M. Teixeira, J.S. Câmara, An improved analytical strategy combining microextraction by packed sorbent combined with ultra high pressure liquid chromatography for the determination of fluoxetine, clomipramine and their active metabolites in human urine, J. Chromatogr. A 1408 (2015) 30-40.

[29] V.C. Jardim, L.P. Melo, D.S. Domingues, M.E.C. Queiroz, Determination of parabens in urine samples by microextraction using packed sorbent and ultra-performance liquid chromatography coupled to tandem 
mass spectrometry, J. Chromatogr. B 974 (2015) 35-41.

[30] I. Moreno, M. Barroso, A. Martinho, A. Cruz, E. Gallardo, Determination of ketamine and its major metabolite, norketamine, in urine and plasma samples using microextraction by packed sorbent and gas chromatography-tandem mass spectrometry, J. Chromatogr. B 1004 (2015) 67-78.

[31] M. Sergi, C. Montesano, S. Odoardi, L.M. Rocca, G. Fabrizi, D. Compagnone, R. Curini, Microextraction by packed sorbent coupled to liquid chromatography tandem mass spectrometry for the rapid and sensitive determination of cannabinoids in oral fluid, J. Chromatogr. A 1301 (2013) 139146.

[32] M. Woźniakiewicz, R. Wietecha-Posłuszny, A. Moos, M. Wieczorek, P. Knihnicki, P. Kościelniak, Development of microextraction by packed sorbent for toxicological analysis of tricyclic antidepressant drugs in human oral fluid, J. Chromatogr. A 1337 (2014) 9-16.

[33] M. Locatelli, M.T. Ciavarella, D. Paolino, C. Celia, E. Fiscarelli, G. Ricciotti, A. Pompilio, G. di Bonaventura, R. Grande, G. Zengin, L. di Marzio, Determination of ciprofloxacin and levofloxacin in human sputum collected from cystic fibrosis patients using microextraction by packed sorbent-high performance liquid chromatography photodiode array detector, J. Chromatogr. A 1419 (2015) 58-66.

[34] R. Said, M. Kamel, A. El-Beqqali, M. Abdel-Rehim, Microextraction by packed sorbent for LC-MS/MS determination of drugs in whole blood samples, Bioanalysis 2 (2010) 197-205. 
[35] R. Said, A. Pohanka, M. Abdel-Rehim, O. Beck, Determination of four immunosuppressive drugs in whole blood using MEPS and LC-MS/MS allowing automated sample work-up and analysis, J. Chromatogr. B 897 (2012) $42-49$.

[36] M.A. Saracino, G. Lazzara, B. Prugnoli, M.A. Raggi, Rapid assays of clozapine and its metabolites in dried blood spots by liquid chromatography and microextraction by packed sorbent procedure, J. Chromatogr. A. 1218 (2011) 2153-2159.

[37] J.L. Gonçalves, V.L. Alves, F.P. Rodrigues, J.A. Figueira, J.S. Câmara, A semi-automatic microextraction in packed sorbent, using a digitally controlled syringe, combined with ultra-high pressure liquid chromatography as a new and ultra-fast approach for the determination of prenyflavonoids in beers, J. Chromatogr. A 1304 (2013) 42-51.

[38] L. Magrini, A. Cappiello, G. Famiglini, P. Palma, Microextraction by packed sorbent (MEPS)-UHPLC-UV: a simple and efficient method for the determination of five benzodiazepines in an alcoholic beverage, J. Pharmaceut. Biomed. 125 (2016) 48-53.

[39] J.M. Leça, V. Pereira, A.C. Pereira, J.C. Marques, Rapid and sensitive methodology for determination of ethyl carbamate in fortified wines using microextraction by packed sorbent and gas chromatography with mass spectrometric detection, Anal. Chim. Acta 811 (2014) 29-35.

[40] R. Perestrelo, C.L. Silva, J.S. Câmara, Quantification of furanic derivatives in fortified wines by a highly sensitive and ultrafast analytical strategy based on digitally controlled microextraction by packed sorbent 
combined with ultrahigh pressure liquid chromatography, J. Chromatogr. A 1381 (2015) 54-63.

[41] J. Gonçalves, C.L. Silva, P.C. Castilho, J.S. Câmara, An attractive, sensitive and high-throughput strategy based on microextraction by packed sorbent followed by UHPLC-PDA analysis for quantification of hydroxybenzoic and hydroxycinnamic acids in wines, Microchem. J. 106 (2013) 129-138.

[42] J. Gonçalves, B. Mendes, C.L. Silva, J.S. Cãmara, Development of a novel microextraction by packed sorbent-bases approach followed by ultrahigh pressure liquid chromatography as a powerful technique for quantification phenolic constituents of biological interest in wines, J. Chromatogr. A 1229 (2012) 13-23.

[43] M.L. Savastano, I. Losito, S. Pati, Rapid and automatable determination of ochratoxin A in wine based on microextraction by packed sorbent followed by HPLC-FLD, Food Control 68 (2016) 391-398.

[44] C. García Pinto, A. Pérez Antón, J.L. Pérez Pavón, B. Moreno Cordero, Coupling of microextraction by packed sorbents with gas chromatography with ionic liquid stationary phases for the determination of haloanisoles in wines, J. Chromatogr. A 1260 (2012) 200-205.

[45] B.H. Fumes, F.N. Andrade, Á.J. Santos Neto, F.M. Lanças, Determination of pesticides in sugarcane juice employing microextraction by packed sorbent followed by gas chromatography and mass spectrometry, J. Sep. Sci. 39 (2016) 2823-2830.

[46] R.K. Heena, R. Kaur, S. Rani, A.K. Malik, Simple and rapid 
determination of phthalates using microextraction by packed sorbent and gas chromatography with mass spectrometry quantification in cold drink and cosmetic samples, J. Sep. Sci. 39 (2016) 923-931.

[47] M. Adam, P. Pavlíková, A. Čížková, P. Bajerová, K. Ventura, Microextraction by packed sorbent (MEPS) as suitable selective method for Lascorbic acid determination in beverages, Food Chem. 135 (2012) 1613-1618.

[48] F.H. Salami, M.E.C. Queiroz, Microextraction in packed sorbent for determination of sulfonamides in egg samples by liquid chromatography and spectrophotometric detection, J. Braz. Chem. Soc. 22 (2011) 1656-1661.

[49] F.H. Salami, M.E.C. Queiroz, Microextraction in packed sorbent for the determination of pesticides in honey samples by gas chromatography coupled to mass spectrometry, J. Chromatogr. Sci. 51 (2013) 899-904.

[50] W. Du, C. Lei, S. Zhang, G. Bai, H. Zhou, M. Sun, Q. Fu, C. Chang, Determination of clenbuterol from pork samples using surface molecularly imprinted polymers as the selective sorbents for microextraction in packed syringe, J. Pharmaceut. Biomed. 91 (2014) 160-168.

[51] M. del Nogal Sánchez, P. Martín Santos, C. Pérez Sappó, J.L. Pérez Pavón, B. Moreno Cordero, Microextraction by packed sorbent and saltingout-assisted liquid-liquid extraction for the determination of aromatic amines formed from azo dyes in textiles, Talanta 119 (2014) 375-384.

[52] A. Prieto, S. Schrader, M. Moeder, Determination of organic priority pollutants and emerging compounds in wastewater and snow samples using multiresidue protocols on the basis of microextraction by packed sorbents coupled to large volume injection gas chromatography-mass spectrometry 
analysis, J. Chromatogr. A 1217 (2010) 6002-6011.

[53] M. Moeder, S. Schrader, U. Winkler, R. Rodil, At-line microextraction by packed sorbent-gas chromatography-mass spectrometry for the determination of UV filter and polycyclic musk compounds in water samples, J. Chromatogr. A 1217 (2010) 2925-2932.

[54] M. del Nogal Sánchez, C. Pérez Sappó, J.L. Pérez Pavón, B. Moreno Cordero, A method based on microextraction by packed sorbent-programmed temperature vaporizer-fast gas chromatography-mass spectrometry for the determination of aromatic amines in environmental water samples, Anal. Bioanal. Chem. 404 (2012) 2007-2015.

[55] G. Grueiro Noche, M.E. Fernández Laespada, J.L. Pérez Pavón, B. Moreno Cordero, Determination of chlorobenzenes in water samples based on fully automated microextraction by packed sorbent coupled with programmed temperature vaporization-gas chromatography-mass spectrometry, Anal. Bioanal. Chem. 405 (2013) 6739-6748.

[56] A.M. Casas Ferreira, M.E. Fernández Laespada, J.L. Pérez Pavón, B. Moreno Cordero, In situ derivatization coupled to microextraction by packed sorbent and gas chromatography for the automated determination of haloacetic acids in chlorinated water, J. Chromatogr. A 1318 (2013) 35-42.

[57] I. González-Mariño, J.B. Quintana, I. Rodríguez, S. Schrader, M. Moeder, Fully automated determination of parabens, triclosan and methyl triclosan in wastewater by microextraction by packed sorbents and gas chromatography-mass spectrometry, Anal. Chim. Acta 684 (2011) 50-66.

[58] A. Prieto, S. Schrader, C. Bauer, M. Moeder, Synthesis of a molecularly 
imprinted polymer and its application for microextraction by packed sorbent for the determination of fluoroquinolone related compounds in water, Anal. Chim. Acta 685 (2011) 146-152.

[59] G. Grueiro Noche, M.E. Fernández Laespada, J.L. Pérez Pavón, B. Moreno Cordero, S. Muniategui Lorenzo, Microextraction by packed sorbent for the analysis of pharmaceutical residues in environmental water samples by in situ derivatization-programmed temperature vaporizer-gas chromatography-mass spectrometry, J. Chromatogr. A 1218 (2011) 93909396.

[60] M.P. Martínez-Moral, M.T. Tena, Use of microextraction by packed sorbents following selective pressurized liquid extraction for the determination of brominated diphenyl ethers in sewage sludge by gas chromatography-mass spectrometry, J. Chromatogr. A 1364 (2014) 28-35.

[61] M. Kaur, S. Rani, A.K. Malik, J. Singh Aulakh, Microextraction by packed sorbent-high pressure liquid chromatographic-ultra violet analysis of endocrine disruptor pesticides in various matrices, J. Chromatogr. Sci. 52 (2014) 977-984. 


\subsection{Reacciones de derivatización}

La utilización de reacciones de derivatización es una estrategia ampliamente utilizada en cromatografía de gases. Las razones predominantes para llevar a cabo este pretratamiento químico son el aumento de la volatilidad de los analitos en estudio, la mejora de las características cromatográficas de los mismos reduciendo su polaridad y/o el aumento de respuesta en el detector [1]. Es deseable que este paso se realice de la forma más rápida posible y con un mínimo número de etapas y reactivos, pero, en muchas ocasiones, se trata de un paso laborioso y que requiere de un elevado tiempo de trabajo.

La naturaleza química, así como la reactividad de los analitos, van a fijar el tipo de derivatización que se ha de llevar a cabo. Nos centraremos en las reacciones más utilizadas para fenoles y aminoácidos, que son los que se han estudiado a lo largo de esta memoria. Se describirá en primer lugar la reacción de acilación de fenoles y, posteriormente la derivatización de aminoácidos con cloroformiatos de alquilo en las que se produce la derivatización simultánea del grupo amino y del grupo carboxilo que los conforman.

\subsubsection{Reacción de acilación de fenoles}

Para los compuestos fenólicos, la reacción más utilizada ha sido la de acilación, ya que se puede llevar a cabo in situ en medio acuoso en pocos minutos, con un rendimiento alto y bajo coste de reactivos. Se han utilizado varios reactivos: cloruro de pentafluorobenzoílo ( $\mathrm{PFBCl}$ ) [2], utilizado específicamente cuando el objetivo es aumentar la sensibilidad al usar un detector de captura electrónica (ECD), cloroformiatos de alquilo [3,4] o 
anhídrido acético en medio básico. Este último es el más utilizado, debido a la rapidez y la alta eficacia con la que transcurre la reacción, incluso a temperatura ambiente [5-7], y es el que se ha utilizado en la realización de esta tesis.

La reacción de acilación con los anhídridos de ácido supone la ruptura del enlace entre el oxígeno y uno de los grupos carbonilos del anhídrido (figura 2.4). Un grupo acilo se transfiere al nucleófilo que ataca; el otro retiene su enlace simple al oxígeno y se convierte en el grupo acilo de un ácido carboxílico.

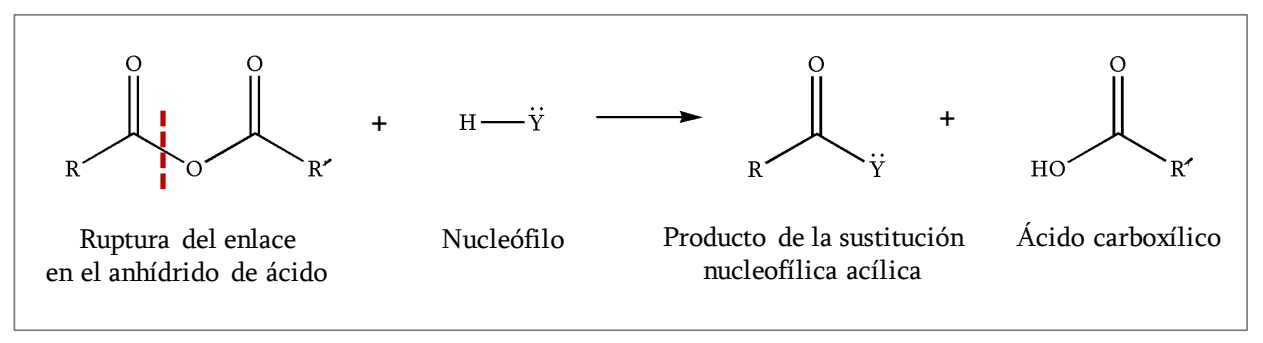

Figura 2.4. Ruptura de un anhídrido de ácido

Los agentes acilantes pueden reaccionar con fenoles (figura 2.5) tanto en el anillo aromático ( $\mathrm{C}$ acilación) como en el oxígeno del hidroxilo ( $\mathrm{O}$ acilación). 


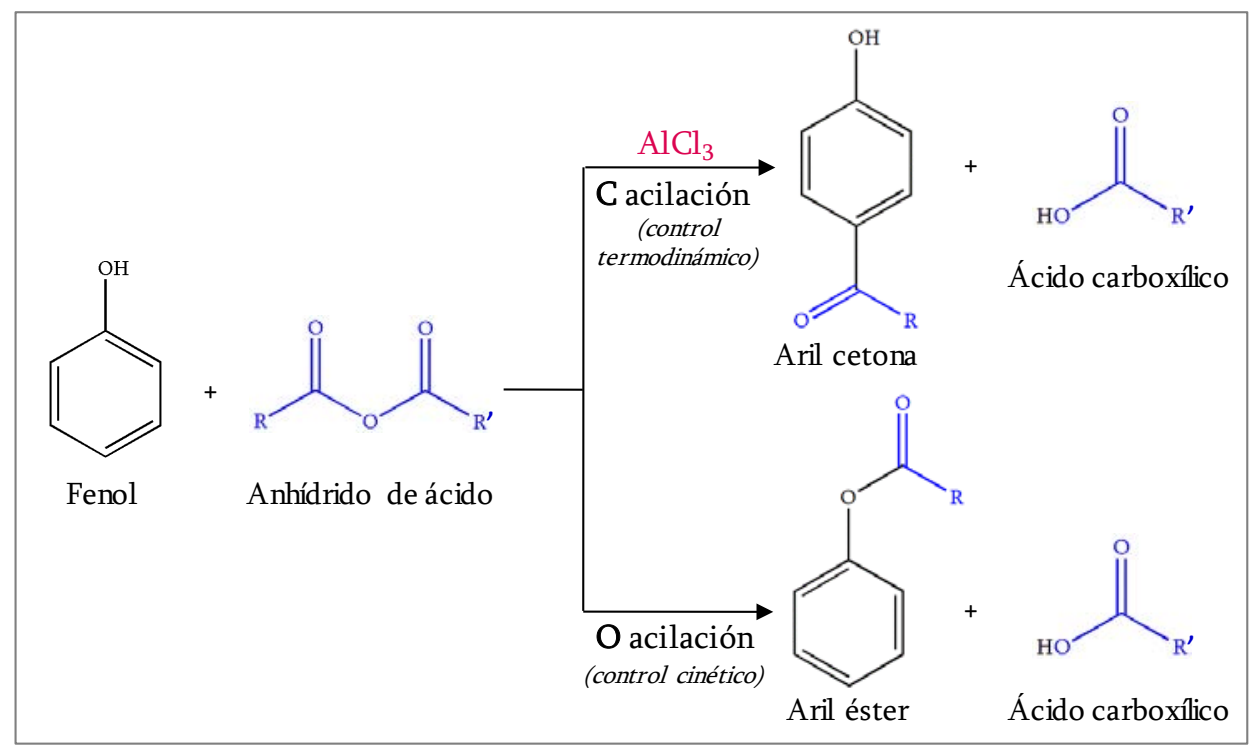

Figura 2.5. Reacción de acilación del fenol

El producto de la $\mathrm{C}$ acilación es más estable y predomina bajo condiciones de control termodinámico, esto es, en presencia de cloruro de aluminio (condiciones habituales de la reacción de Friedel-Crafts). Sin embargo, el producto de la $\mathrm{O}$ acilación se forma más rápido y predomina bajo condiciones de control cinético (sin cloruro de aluminio).

La reacción de $\mathrm{O}$ acilación puede ser favorecida por dos caminos (figura 2.6). Un método supone convertir el anhídrido de ácido en un agente de transferencia de acilo más efectivo, es decir, aumentar su carácter electrófilo por protonación de uno de sus oxígenos carboxílicos con la adición de unas pocas de gotas de ácido sulfúrico. Otra alternativa es aumentar el carácter nucleófilo del fenol, convirtiéndolo en su anión fenóxido en disolución básica. 


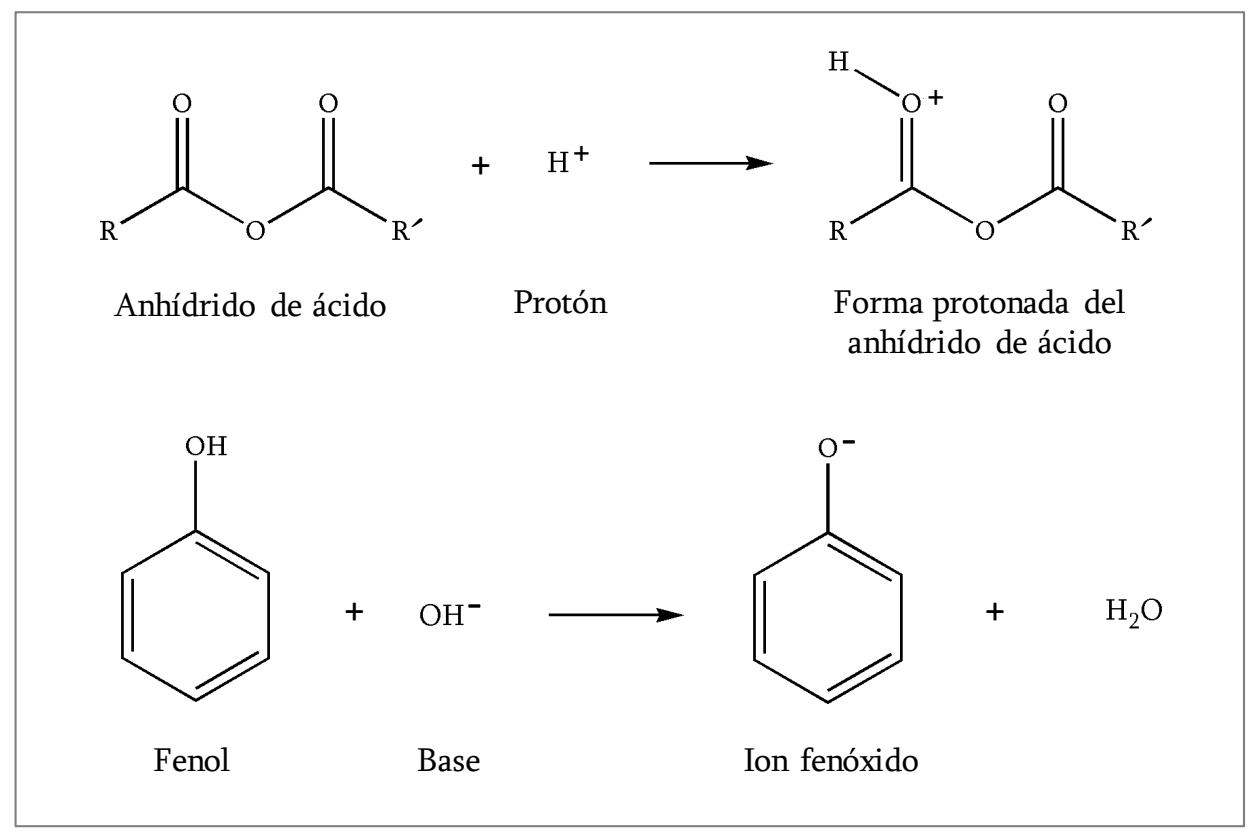

Figura 2.6. Posibilidades de favorecer la reacción

La opción más utilizada para llevar a cabo la $\mathrm{O}$ acilación es el uso de medio básico proporcionado por carbonato potásico o sódico [8-12], hidrogenocarbonato potásico o sódico $[5,13,15,16]$, hidrogenofosfato potásico o sódico [16-21] o hidróxido sódico [22-24]. También se ha propuesto el uso de óxido de grafito [25] así como de otros materiales y compuestos [26-29] para catalizar la reacción sin necesidad de usar medio ácido o básico.

Los ésteres formados son moderadamente polares. Dado que carecen de grupos hidroxilo, las moléculas de éster no pueden formar enlaces por puentes de hidrógeno unos con otros; consecuentemente, los ésteres tienen puntos de ebullición menores que los alcoholes de peso molecular comparable. También son bastante estables en medio acuoso neutro, pero se hidrolizan cuando se calientan en medio ácido o básico. 
En la presente memoria se ha utilizado la reacción de formación de ésteres con anhídrido acético en medio básico para la determinación de clorofenoles en muestras de suelo (I.2).

\subsubsection{Derivatización de aminoácidos con cloroformiatos de alquilo}

El tratamiento de aminoácidos con cloroformiato de isobutilo fue propuesto por primera vez en la década de los 70 por Makita y colaboradores [30-32]. El procedimiento consistía en dos pasos en el que primero se producía el carbamato procedente de la reacción del grupo amino con cloroformiato de isobutilo y seguidamente se llevaba a cabo la metilación del grupo carboxilo con diazometano, para lo que era necesaria una extracción entre ambas etapas para eliminar el exceso de reactivos.

Posteriormente, los mismos autores propusieron numerosas aplicaciones y modificaciones de este procedimiento con el fin de mejorarlo [33-36]. También se propuso la sililación como alternativa a la metilación del grupo carboxilo en la segunda etapa del procedimiento [37].

La reacción de los cloroformiatos de alquilo con los grupos amino (figura 2.7) y fenólicos en medio acuoso para dar carbamatos y carbonatos, respectivamente, era bien conocida y utilizada hasta ese momento. 


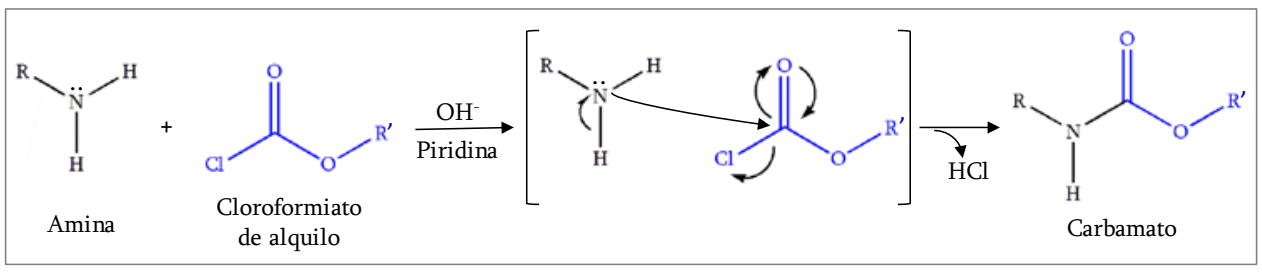

Figura 2.7. Mecanismo de formación de carbamato a partir de amina y cloroformiato de alquilo [38]

En cuanto a la reacción de los cloroformiatos con los grupos carboxilo, se sabía que daba lugar a la formación de los llamados anhídridos mixtos y que éstos, a su vez, podían transformarse en ésteres en presencia de un catalizador bajo ciertas condiciones de reacción $[39,40]$ o mediante la reacción (también catalizada) con un alcohol [41,42], siempre en medios no acuosos. Sin embargo, nada se sabía de la capacidad de los cloroformiatos para actuar como agentes de esterificación de los grupos carboxilo en medio acuoso y, dados los resultados poco alentadores obtenidos en medios orgánicos, su aplicación en procedimientos de derivatización para cromatografía de gases no era todavía posible [43].

Fue a principios de la década de los 90 cuando Hušek consiguió la esterificación del grupo carboxilo de los ácidos grasos con cloroformiatos de alquilo en medio acuoso en presencia de un alcohol y de piridina como catalizador [44]. A la vista de los resultados obtenidos con los grupos carboxilo en su trabajo y teniendo en cuenta la ya conocida reacción de los cloroformiatos con los grupos amino, Hušek introdujo en el año 1991 la derivatización de aminoácidos en un solo paso con cloroformiato de etilo [4547], en la que se conseguía por primera vez la derivatización simultánea de 
ambos grupos funcionales. Esta derivatización consiste en el tratamiento de los aminoácidos con una mezcla de cloroformiato, alcohol y piridina para obtener los $\mathrm{N}(\mathrm{O}, \mathrm{S})$-alquil alcoxicarbonil ésteres de aminoácidos. Este método de derivatización ofrece muchas ventajas para la determinación de aminoácidos mediante cromatografía de gases, ya que se produce en medio acuoso, a temperatura ambiente en condiciones suaves y prácticamente es instantánea.

En referencia al mecanismo de reacción que sigue el grupo carboxilo al reaccionar con los cloroformiatos, Hušek propuso que el anhídrido mixto formado de la reacción de ambos debía descarboxilarse $\left(-\mathrm{CO}_{2}\right)$ para producir el éster que contenía el grupo alquilo derivado del cloroformiato de alquilo. Sin embargo, un estudio posterior de Wang y colaboradores [48] en el que se hacían reaccionar los aminoácidos con diferentes combinaciones de cloroformiatos de alquilo y alcoholes, puso de manifiesto que el anhídrido mixto reaccionaba con el alcohol para dar lugar al éster que contiene el grupo alquilo procedente de este alcohol (figura 2.8). 


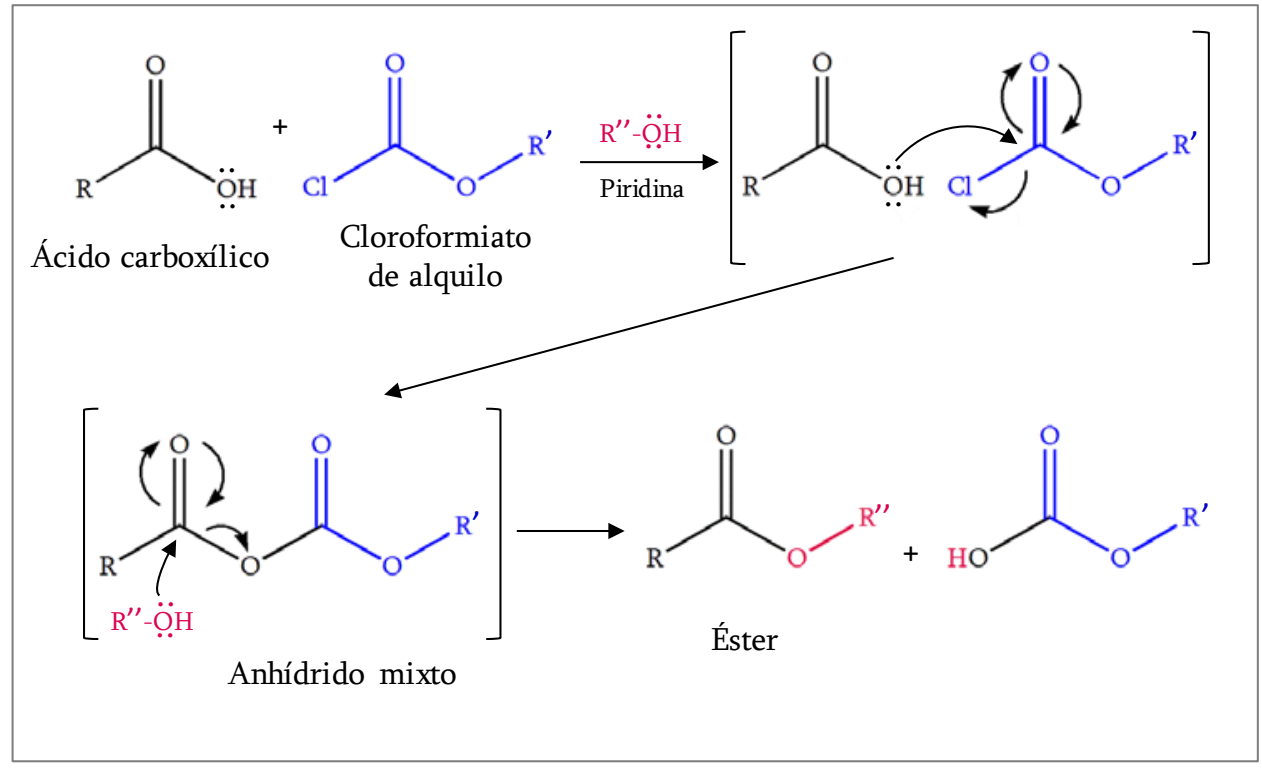

Figura 2.8. Mecanismo de reacción de un ácido carboxílico y un cloroformiato de alquilo [38]

Esta sería la vía de reacción conducente al producto mayoritario cuando se hace reaccionar un grupo carboxilo con cloroformiato en presencia de alcohol y piridina (figura 2.9 vía A). No obstante, también se encontró como producto de esta reacción una pequeña cantidad del éster con el mismo grupo alquilo que el del cloroformiato que se había utilizado como reactivo de la reacción. Este producto minoritario podría proceder de dos posibles rutas. Una vía sería la descarboxilación del anhídrido mixto (figura 2.9 vía B), mientras que la otra plantea la posibilidad de que una pequeña cantidad de alcohol producido in situ por la hidrólisis del cloroformiato de alquilo reaccionara con el anhídrido mixto (figura 2.9 vía C). 


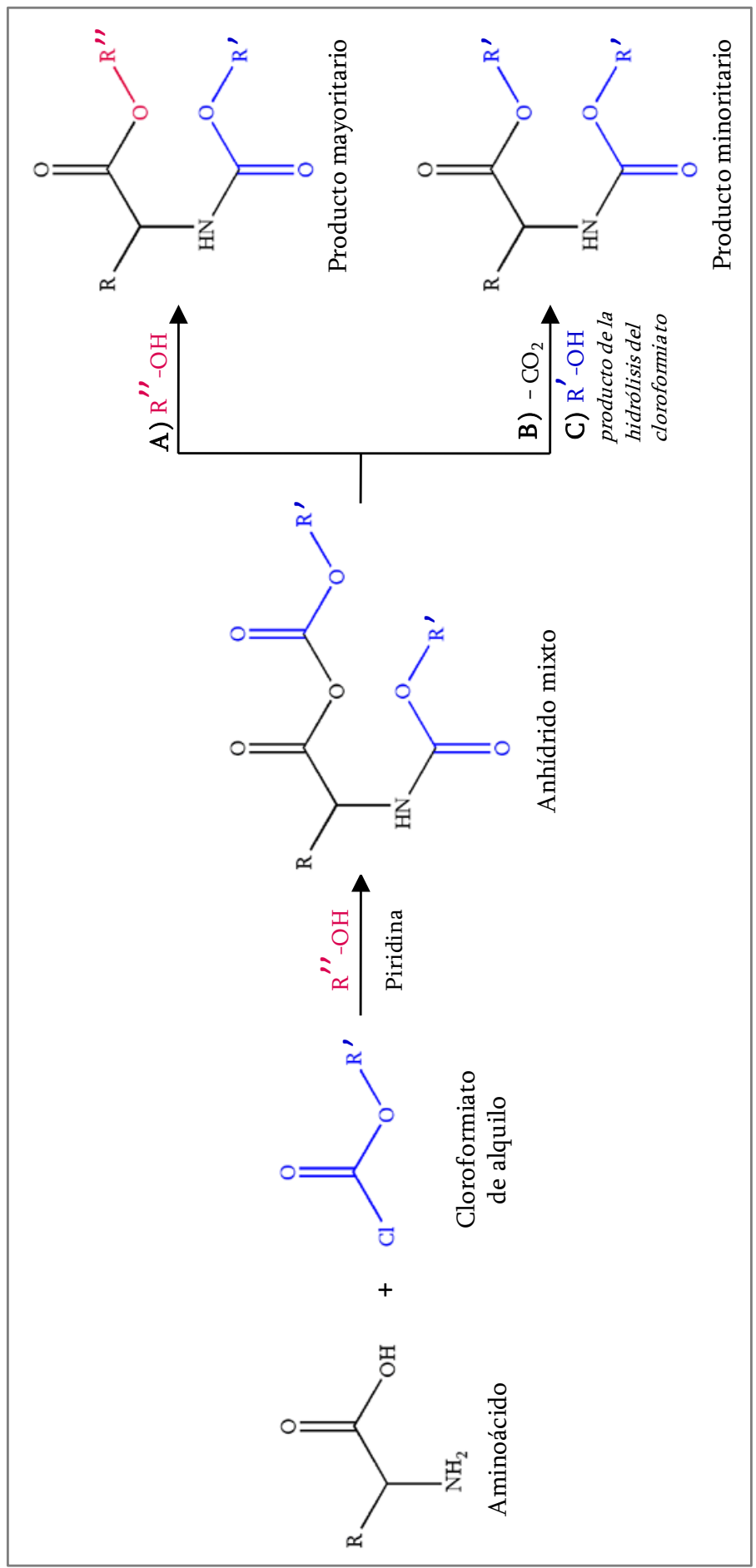

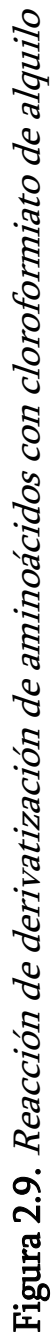


Pese a que la obtención de dos productos de reacción parezca en un principio un inconveniente para su aplicación, existe una solución fácil para evitarlo que consiste en la elección del mismo grupo alquilo en el alcohol y en el cloroformiato, para obtener así el mismo producto mediante ambas vías. De este modo, el cloroformiato de metilo iría acompañado de metanol, el cloroformiato de etilo de etanol, y así sucesivamente.

Debido a la simplicidad y rapidez del procedimiento, así como a la pronta caracterización de los patrones de fragmentación de los derivados en el espectrómetro de masas [49], la reacción de aminoácidos con cloroformiatos se extendió rápidamente como técnica de derivatización para la determinación de estos compuestos mediante cromatografía de gases [50,51]. Son muchos los trabajos que se han publicado desde entonces empleando diferentes cloroformiatos como cloroformiato de metilo [52-55], cloroformiato de etilo [56-59], cloroformiato de propilo [60,61] o cloroformiato de isobutilo [62-64], en diferentes muestras biológicas y medioambientales.

En el método propuesto en esta memoria (II.1) se ha utilizado cloroformiato de etilo con etanol y piridina para la derivatización de aminoácidos en muestras de orina.

\section{Bibliografía}

[1] R.J. Wells, Recent advances in non-silylation derivatization techniques for gas chromatography, J. Chromatogr. A 843 (1999) 1-18. 
[2] F. Bianchi, M. Careri, C. Mucchino, M. Musci, Improved determination of chlorophenols in water by solid-phase microextraction followed by benzoylation and gas chromatography with electron capture detection, Chromatographia 55 (2002) 595-600.

[3] V. Maurino, C. Minero, E. Pelizzetti, S. Angelino, M. Vicenti, Ultratrace determination of highly hydrophilic compounds by 2,2,3,3,4,4,5,5octafluoropentyl chloroformate-mediated derivatization directly in water, J. Am. Soc. Mass Spectrom. 10 (1999) 1328-1336.

[4] M. Vicenti, S. Biazzi, N. Ghiglione, M.C. Valsania, S.D. Richardson, Comparison of highly-fluorinated chloroformates as direct aqueous sample derivatization agents for hydrophilic analytes and drinking-water disinfection by-products, J. Am. Soc. Mass Spectrom. 16 (2005) 803-813.

[5] M. Llompart, M. Lourido, P. Landín, C. García-Jares, R. Cela, Optimization of a derivatization-solid phase microextraction method for the analysis of thirty phenolic pollutants in water samples, J. Chromatogr. A 963 (2002) 137-148.

[6] I. Turnes, I. Rodríguez, C.M. García, R. Cela, Determination of chlorophenols in drinking water with high resolution gas chromatographytandem mass spectrometry, J. Chromatogr. A 743 (1996) 283-292.

[7] J. Regueiro, E. Becerril, C. García-Jares, M. Llompart, Trace analysis of parabens, triclosan and related chlorophenols in water by headspace solidphase microextraction with in situ derivatization and gas chromatographytandem mass spectrometry, J. Chromatogr. A 1216 (2009) 4693-4702. 
[8] R. Morales, M.C. Ortiz, L.A. Sarabia, Optimization of headspace experimental factors to determine chlorophenols in water by means of headspace solid-phase microextraction and gas chromatography coupled with mass spectrometry and parallel factor analysis, Anal. Chim. Acta 754 (2012) 20-30.

[9] F. Galán-Cano, R. Lucena, S. Cárdenas, M. Valcárcel, Ionic liquid based in situ solvent formation microextraction coupled to thermal desorption for chlorophenols determination in waters by gas chromatography/mass spectrometry, J. Chromatogr. A 1229 (2012) 48-54.

[10] H. Faraji, M.S. Tehrani, S.W. Husain, Pre-concentration of phenolic compounds in water samples by novel liquid-liquid microextraction and determination by gas chromatography-mass spectrometry, J. Chromatogr. A 1216 (2009) 8569-8574.

[11] D. Budziak, E. Martendal, E. Casarek, Application of NiTi alloy coated with $\mathrm{ZrO}_{2}$ as a new fiber for solid-phase microextraction for determination of halophenols in water samples, Anal. Chim. Acta 598 (2007) 254-260.

[12] J.I. Cacho, N. Campillo, P. Viñas, M. Henández-Córdoba, Stir bar sorptive extraction coupled to gas chromatography-mass spectrometry for the determination of bisphenols in canned beverages and filling liquids of canned vegetables, J. Chromatogr. A 1247 (2012) 146-153.

[13] J.L. Pérez Pavón, A.M. Casas Ferreira, M.E. Fernández Laespada, B. Moreno Cordero, Use of a programmed temperature vaporizer and an in situ derivatization reaction to improve sensitivity in headspace-gas 
chromatography. Application to the analysis of chlorophenols in water, J. Chromatogr. A 1216 (2009) 1192-1199.

[14] E. Baltussen, F. David, P. Sandra, H. Janssen, C. Cramers, Automated sorptive extraction-thermal desorption-gas chromatography-mass spectrometry analysis: determination of phenols in water samples, J. Microcolumn. Sep. 11 (1999) 471-474.

[15] A.M. Casas Ferreira, M. Möder, M.E. Fernández Laespada, GC-MS determination of parabens, triclosan and methyl triclosan in water by in situ derivatization and stir-bar sorptive extraction, Anal. Bioanal. Chem. 399 (2011) 945-953.

[16] T. Rodríguez-Cabo, I. Rodríguez, R. Cela, Determination of hydroxylated stilbenes in wine by dispersive liquid-liquid microextraction followed by gas chromatography mass spectrometry, J. Chromatogr. A 1258 (2012) 21-19.

[17] A. Prichodko, E. Janenaite, V. Smitiene, V. Vickackaite, Gas chromatographic determination of parabens after in situ derivatization and dispersive liquid-liquid microextraction, Acta Chromatogr. 24 (2012) 589-601.

[18] E. Priego-López, M.D. Luque de Castro, Ultrasound-assisted derivatization of phenolic compounds in spiked water samples before pervaporation, gas chromatography separation, and flame ionization detection, Chromatographia 57 (2003) 513-518.

[19] N. Ramírez, F. Borrull, R.M. Marcé, Simultaneous determination of parabens and synthetic musks in water by stir-bar sorptive extraction and 
thermal desorption-gas chromatography-mass spectrometry, J. Sep. Sci. 35 (2012) 580-588.

[20] J. Regueiro, E. Becerril, C. García-Jares, M. Llompart, Trace analysis of parabens, triclosan and related chlorophenols in water by headspace solidphase microextraction with in situ derivatization and gas chromatographytandem mass spectrometry, J. Chromatogr. A 1216 (2009) 4693-4702.

[21] J. Regueiro, M. Llompart, E. Psillakis, J.C. García-Moteagudo, C. GarcíaJares, Ultrasound-assisted emulsification-microextraction of phenolic preservatives in water, Talanta 79 (2009) 1387-1397.

[22] R. Ito, M. Kawaguchi, H. Honda, Y. Koganei, N. Okanouchi, N. Sakui, K. Saito, H. Nakazawa, Hollow-fiber-supported liquid phase microextraction with in situ derivatization and gas chromatography-mass spectrometry for determination of chlorophenols in human urine samples, J. Chromatogr. B 872 (2008) 63-97.

[23] M. Kawaguchi, R. Ito, N. Okanouchi, K. Saito, H. Nakazawa, Miniaturized hollow fiber assisted liquid-phase microextraction with in situ derivatization and gas chromatography-mass spectrometry for analysis of bisphenol A in human urine samples, J. Chromatogr. B 870 (2008) 98-102.

[24] R. Zhao, J. Yuan, H. Li, X. Wang, T. Jiang, J. Lin, Nonequilibrium hollow-fiber liquid-phase microextraction with in situ derivatization for the measurement of triclosan in aqueous samples by gas chromatography-mass spectrometry, Anal. Bioanal. Chem. 387 (2007) 2911-2915.

[25] Y. Liu, J. Qi, L. Bai, Y. Xu, N. Ma, F. Sun, Graphite oxide-catalyzed acetylation of alcohols and phenols, Chinese Chem. Lett. 27 (2016) 726-730. 
[26] M. Seddighi, F. Shirini, O. Goli-Jolodar, Preparation, characterization and application of RHA/TiO2 nanocomposites in the acetylation of alcohols, phenols and amines, C. R. Chimie 19 (2016) 1003-1010.

[27] M. Alam, A. Rahman, N.M. Alandis, M.R. Shaik, Ni/Silica catalyzed acetylation of phenols and naphthols: An eco-friendly approach, Arab. J. Chem. 7 (2014) 53-56.

[28] S. Farhadi, K. Jahanara, $\mathrm{ZnAl}_{2} \mathrm{O}_{4} @ \mathrm{SiO}_{2}$ nanocomposite catalyst for the acetylation of alcohols, phenols and amines with acetic anhydride under solvent-free conditions, Chinese J. Catal. 35 (2014) 368-375.

[29] F.M. Moghaddam, M. Doulabi, H. Saeidian, Controlled microwaveassisted synthesis of $\mathrm{ZnFe}_{2} \mathrm{O}_{4}$ nanoparticles and their catalytic activity for Oacylation of alcohol and phenol in acetic anhydride, Scientia Iranica C 19 (6) (2012) 1597-1600.

[30] M. Makita, S. Yamamoto, M. Kono, K. Sakai, M. Shiraishi, Chem. Ind. (London) 19 (1975) 355.

[31] M. Makita, S. Yamamoto, M. Kono, Gas-liquid chromatographic analysis of protein amino acids as $\mathrm{N}$-isobutyloxycarbonylamino acid methyl esters, J. Chromatogr. 120 (1976) 129-140.

[32] M. Makita, S. Yamamoto, S. Kiyama, Improved gas-liquid chromatographic method for the determination of protein amino acids, J. Chromatogr. 237 (1982) 279-284. 
[33] H. Katoaka, N. Sakiyama, M. Makita, Gas chromatographic analysis of aminoalkylphosphonic acids and aminoalkyl phosphates, J. Chromatogr. 436 (1988) 67-72.

[34] H. Katoaka, Y. Ueno, M. Makita, Analysis of O-phosphoamino acids in proteins by gas chromatography with flame photometric detection, Agric. Biol. Chem. 55 (1991) 1587-1592.

[35] H. Katoaka, K. Takagi, M. Makita, Determination of total plasma homocysteine and related aminothiols by gas chromatography with flame photometric detection, J. Chromatogr. B 664 (1995) 421-425.

[36] S. Matsumura, H. Kataoka, M. Makita, Capillary gas chromatographic analysis of protein amino acids as their $\mathrm{N}(\mathrm{O}, \mathrm{S})$-isobutoxycarbonyl methyl ester derivatives, Biomed. Chromatogr. 9 (1995) 205-210.

[37] K.R. Kim, J.H. Kim, C.H. Oh, T.J. Mabry, Capillary gas chromatography of protein amino acids $\mathrm{N}(\mathrm{O}, \mathrm{S})$-isobutyloxycarbonyl tert-butyldimethylsilyl derivatives in aqueous samples, J. Chromatogr. 605 (1992) 241-249.

[38] K.F. Smart, R.B.M. Aggio, J.R. Van Houtte, S.G. Villas-Bôas, Analytical platform for metabolome analysis of microbial cells using methyl chloroformate derivatization followed by gas chromatography-mass spectrometry, Nat. Protoc. 5 (2010) 1709-1729.

[39] S. Kim, Y.C. Kim, J.I. Lee, A new convenient method for the esterification of carboxyl acids, Tetrahedron Lett. 24 (1983) 3365-3368. 
[40] S. Kim, J.I. Lee, Y.C. Kim, A simple and mild esterification method for carboxylic acids using mixed carboxylic-carbonic anhydrides, J. Org. Chem. 50 (1985) 560-565.

[41] J. Inanaga, K. Hirata, H. Saeki, T. Katsuki, M. Yamaguchi, A rapid esterification by means of mixed anhydride and its application to large-ring lactonization, Bull. Chem. Soc. Japan 52 (1979) 1989-1993.

[42] C. Zeggaf, J. Poncet, P. Jouin, M.N. Dofour, B. Castro, Isopropenyl chlorocarbonate (IPPC) in amino acid and peptide chemistry: esterification of $\mathrm{N}$-protected amino acids; application to the synthesis of the depsipeptide valinomycin, Tetrahedron 45 (1989) 5039-5050.

[43] P. Hušek, Chloroformates in gas chromatography as general purpose derivatizing agents, J. Chromatogr. B 717 (1998) 57-91.

[44] P. Hušek, J.A. Rijks, P.A. Leclercq, C.A. Cramers, Fast esterification of fatty acids with alkyl chloroformates, J. High Resolut. Chromatogr. 13 (1990) 633-638.

[45] P. Hušek, Amino acid derivatization and analysis in five minutes, FEBS Lett. 280 (1991) 354-356.

[46] P. Hušek, C.C. Sweeley, Gas chromatographic separation of protein amino acids in four minutes, J. High Resolut. Chromatogr. 14 (1991) 751-753.

[47] P. Hušek, Rapid derivatization and gas chromatographic determination of amino acids, J. Chromatogr. 552 (1991) 289-299.

[48] J. Wang, Z-H. Huang, D.A. Gage, J.T. Watson, Analysis of amino acids by gas chromatography-flame ionization detection and gas chromatography- 
mass spectrometry: simultaneous derivatization of functional groups by an aqueous-phase chloroformate-meditated reaction, J. Chromatogr. A 663 (1994) 71-78.

[49] Z-H. Huang, J. Wang, D.A. Gage, J.T. Watson, C.C. Sweely, P. Hušek, Characterization of N-ethoxycarbonyl ethyl esters of amino acids by mass spectrometry, J. Chromatogr. 635 (1993) 271-281.

[50] P. Hušek, en: I. Molnár-Perl (Ed.), Quantitation of amino acids as chloroformates: a return to gas chromatography, Elsevier, Amsterdam, 2005, p. 2.

[51] P. Hušek, P. Simek, Alkyl chloroformates in sample derivatization strategies for GC analysis review on a decade use of the reagents as esterifying agents, Curr. Pharm. Anal. 2 (2006) 23-43.

[52] B. Vancompernolle, K. Croes, G. Angenon, Optimization of a gas chromatography-mass spectrometry method with methyl chloroformate derivatization for quantification of amino acids in plant tissue, J. Chromatogr. B 1017 (2016) 241-249.

[53] W-P. Chen, X-Y. Yang, A.D. Hegeman, W.M. Gray, J.D. Cohen, Microscale analysis of amino acids using gas chromatography-mass spectrometry after methyl chloroformate derivatization, J. Chromatogr. B 878 (2010) 2199-2208.

[54] S.G. Villas-Bôas, D.G. Delicado, M. Akesson, J. Nielsen, Simultaneous analysis of amino and nonamino organic acids as methyl chloroformate derivatives using gas chromatography-mass spectrometry, Anal. Biochem. 322 (2003) 134-138. 
[55] C. Rodier, R. Sternberg, F. Raulin, C. Vidal-Madjar, Chemical derivatization of amino acids for in situ analysis of Martian samples by gas chromatography, J. Chromatogr. A 915 (2001) 199-207.

[56] M.K.R. Mudiam, Ch. Ratnasekhar, Ultra sound assisted one step rapid derivatization and dispersive liquid-liquid microextraction followed by gas chromatography-mass spectrometric determination of amino acids in complex matrices, J. Chromatogr. A 1291 (2013) 10-18.

[57] M.K.R. Mudiam, Ch. Ratnasekhar, R. Jain, P.N. Saxena, A. Chauhan, R.C. Murthy, Rapid and simultaneous determination of twenty amino acids in complex biological and food samples by solid-phase microextraction and gas chromatography-mass spectrometry with the aid of experimental design after ethyl chloroformate derivatization, J. Chromatogr. B 907 (2012) 56-64.

[58] X. Gao, E. Pujos-Guillot, J-F. Martin, P. Galan, C. Juste, W. Jia, J-L. Sebedio, Metabolite analysis of human fecal water by gas chromatography/mass spectrometry with ethyl chloroformate derivatization, Anal. Biochem. 393 (2009) 163-175.

[59] Y. Qiu, M. Su, Y. Liu, M. Chen, J. Gu, J. Zhang, W. Jia, Application of ethyl chloroformate derivatization for gas chromatography-mass spectrometry based metabonomic profiling, Anal. Chim. Acta 583 (2007) 277283.

[60] E. Gionfriddo, A. Naccarato, G. Sindona, A. Tagarelli, A reliable solid phase microextraction-gas chromatography-triple quadrupole mass spectrometry method for the assay of selenomethionine and 
selenomethylselenocysteine in aqueous extracts: difference between selenized and not-enriched selenium potatoes, Anal. Chim. Acta 747 (2012) 58-66.

[61] S. Kawana, K. Nakagawa, Y. Hasegawa, S. Yamaguchi, Simple and rapid analytical method for detection of amino acids in blood using blood spot on filter paper, fast-GC/MS and isotope dilution technique, J. Chromatogr. B 878 (2010) 3113-3118.

[62] T.G. Sobolevsky, A.I. Revelsky, I.A. Revelsky, B. Miller, V. Oriedo, Simultaneous determination of fatty, dicarboxylic and amino acids based on derivatization with isobutyl chloroformate followed by gas chromatographypositive ion chemical ionization mass spectrometry, J. Chromatogr. B 800 (2004) 101-107.

[63] C. Deng, N. Li, X. Zhang, Rapid determination of amino acids in neonatal blood samples based on derivatization with isobutyl chloroformate followed by solid-phase microextraction and gas chromatography/mass spectrometry, Rapid Commun. Mass Spectrom. 18 (2004) 2558-2564.

[64] Y. Shinohara, H. Hasegawa, K. Tagoku, T. Hashimoto, Simultaneous determination of methionine and total homocysteine in human plasma by gas chromatography-mass spectrometry, J. Chromatogr. B 758 (2001) 283-288. 


\subsection{Generación de espacio de cabeza}

La generación de espacio de cabeza (HS) es una técnica utilizada para la separación de los compuestos volátiles de una muestra. Se denomina espacio de cabeza a la fase gaseosa en contacto con la muestra (líquida o sólida). Puede acoplarse a diversas técnicas analíticas de separación y medida, pero se ha utilizado principalmente acoplada a cromatografía de gases (GC), debido a que ésta es una técnica muy apropiada para el análisis de fases gaseosas [1].

El procedimiento de generación del espacio de cabeza puede llevarse a cabo de forma estática y dinámica [2], siendo la forma estática la alternativa más sencilla, con mayor grado de automatización y la más rápida.

En HS estático (static headspace sampling, SHS) la muestra se introduce en un vial dejando un volumen libre sobre ella. El vial se cierra herméticamente y se introduce en un horno, de modo que los volátiles se separan de la matriz y al cabo de un tiempo se establece el equilibrio entre ambas fases. Una vez alcanzado el equilibrio, una porción de los volátiles generados se inyecta en el cromatógrafo de gases.

A partir de esta modalidad, a la que también se ha denominado HS "en un paso", se han desarrollado diversas modificaciones, entre las que pueden citarse la inclusión de trampas de adsorción o de trampas que contienen un disolvente orgánico, cuyo propósito es separar los analitos volátiles de interés del resto de los compuestos de la fase gaseosa.

En 1989 Pawliszyn y su grupo de investigación introdujeron la técnica de microextracción en fase sólida (SPME) [3,4]. Una fibra de sílice fundida, cuya superficie externa está recubierta con una fase estacionaria inmovilizada, 
se introduce en el vial que contiene la muestra. Al cabo de un tiempo los volátiles quedan adsorbidos en la fibra, ésta se introduce en la cámara de vaporización del inyector del cromatógrafo de gases y los analitos son transferidos a la columna cromatográfica por desorción térmica. La fibra puede sumergirse en el líquido o permanecer en el espacio de cabeza sobre la muestra líquida o sólida. En el caso en que esta técnica se utilice para extraer los volátiles del espacio de cabeza de la muestra se denomina HS-SPME. Este acoplamiento se utilizó por primera vez en 1993 [5] y desde entonces ha sido empleado para resolver numerosos problemas analíticos.

La modalidad SHS también se ha utilizado combinada con microextracción en fase líquida (liquid-phase microextraction, LPME) [6] en la que una gota de disolvente orgánico en la punta de una microjeringa se sitúa suspendida sobre la muestra durante el proceso de generación de espacio de cabeza, de manera que se establece un equilibrio de distribución de los analitos entre la fase gaseosa y el disolvente orgánico. Finalmente, el disolvente con los analitos preconcentrados se inyecta en el cromatógrafo de gases para proceder a su análisis.

El modo de generación de espacio de cabeza dinámico fue sugerido por primera vez en 1962 por Swinnerton y colaboradores [7,8]. Bellar y Lichtenberg [9] desarrollaron la técnica y la denominaron Purga y Trampa (P\&T). En esta modalidad no se establece el equilibrio entre las dos fases del vial, sino que la separación de los volátiles se lleva a cabo retirando continuamente la fase gaseosa, hasta que todos los analitos volátiles han sido extraídos de la muestra. Se utiliza un flujo de gas inerte sobre la muestra sólida o líquida, o bien se hace burbujear el gas a través de la muestra líquida. Los 
volátiles purgados están diluidos en el gas extractante y es necesario focalizarlos en una trampa antes de introducirlos en la columna cromatográfica. Esta focalización puede hacerse mediante trampa fría, aunque generalmente se ha utilizado un cartucho empaquetado con un material adsorbente, a partir del cual los volátiles se transfieren a la columna cromatográfica por desorción térmica.

La generación de espacio de cabeza en ambas modalidades, permite analizar los compuestos volátiles de una muestra sin interferencias de los compuestos no volátiles de la matriz. Se trata de un proceso de extracción sencillo, que minimiza la etapa de pretratamiento de la muestra, reduciendo considerablemente los errores asociados a la misma, el tiempo y el coste del análisis.

La sensibilidad de la metodología HS-GC depende, además del detector, de la capacidad de la columna cromatográfica [10]. El espacio de cabeza está constituido por una muestra gaseosa más o menos diluida. El problema que se plantea al introducir la muestra en el cromatógrafo de gases es cómo introducir el mayor volumen de muestra posible, para alcanzar los niveles de sensibilidad deseados y, además, hacerlo de forma rápida para reducir el ancho de banda inicial del cromatograma.

En el libro publicado por el grupo de investigación de Bruno Kolb [1] se describen algunas técnicas que se han empleado para solucionar los problemas planteados. Se trata de técnicas de enriquecimiento por trampa criogénica. Con estas técnicas se consigue la preconcentración de los analitos y su posterior introducción rápida en la columna cromatográfica. 
Los primeros dispositivos consistían en trampas con forma de $\mathrm{U}$, fabricados con metal o vidrio, que se introducían en un baño de nitrógeno líquido o argón. Una vez conseguida la condensación de los analitos en el interior del tubo, el líquido criogénico era retirado manualmente del baño antes de que la trampa fuera calentada, en la mayoría de los casos eléctricamente, para conseguir la evaporación de los analitos condensados. Posteriormente, surgen algunos avances para evitar la eliminación manual del líquido criogénico. En lugar de un baño se utiliza un tubo de teflón que rodea la trampa en forma de $\mathrm{U}$ y un flujo de agua caliente desplaza al nitrógeno líquido una vez finalizada la preconcentración de los compuestos.

Más adelante surgen trampas en las que el enfriamiento se consigue mediante una corriente de gas y la posterior vaporización de los analitos sustituyendo el flujo de gas frío por uno de gas caliente. Con el fin de evitar la corriente de gas caliente necesaria para conseguir la vaporización, surge la idea de introducir el dispositivo en el interior del cromatógrafo de gases, de modo que, una vez que el flujo de gas frío deja de circular, la trampa adquiere rápidamente la temperatura del horno del GC.

Esta estrategia se ha llevado a cabo, principalmente, enfriando la parte inicial de la columna cromatográfica mediante una corriente de nitrógeno gas (previamente enfriado en el exterior del cromatógrafo), que se hace circular a través de un tubo de teflón que rodea la parte inicial de la columna. También se han utilizado flujos de $\mathrm{N}_{2}$ líquido o $\mathrm{CO}_{2}$ líquido. La muestra condensa en cabeza de columna y posteriormente se aumenta progresivamente la temperatura para que el disolvente eluya rápidamente, mientras que los analitos, con mayor punto de ebullición, permanecen en una banda estrecha. 
Este sistema tiene algunas limitaciones. En primer lugar, el flujo a través de la región enfriada está limitado por el flujo en columna. En segundo lugar, el atrapamiento en cabeza de columna a baja temperatura no retiene únicamente los analitos, sino que también se atrapan impurezas o materiales indeseables, por lo que frecuentemente hay que cortar secciones de columna para eliminar este material interferente. Por último, cuando la preconcentración se produce a temperatura bajo cero pueden producirse problemas de bloqueo del flujo en columnas capilares por congelación de agua [11-13].

Una alternativa es el uso de pre-columnas, que consisten en una sección de columna cromatográfica desactivada que se conecta a la parte inicial de la columna en la que se va a realizar la separación de los compuestos. Estos dispositivos son más fácilmente reemplazables, aunque requieren una conexión apropiada a la columna cromatográfica.

Además del bloqueo del flujo en columna por formación de hielo, la presencia de agua en GC puede dar lugar a distorsión de la forma de pico, particularmente, de aquellos compuestos con menor tiempo de retención que eluyen junto con el agua. Para resolverlo se han desarrollado diversas técnicas para eliminar el exceso de vapor de agua antes de introducir la muestra en la columna cromatográfica. En la técnica de HS dinámico se utilizan membranas semipermeables y condensadores de reflujo. Cuando se utiliza HS estático, el volumen de vapor de agua generado es menor y suele eliminarse mediante adsorción química selectiva sobre una sal inerte higroscópica [10].

El principal problema de las trampas criogénicas utilizadas es que suelen ser de fabricación manual y requieren una alta cualificación para ser 
manejadas de forma adecuada. Esto pone de manifiesto la necesidad de dispositivos comerciales y fácilmente automatizables que sean capaces de solucionar los problemas del acoplamiento HS-GC, para conseguir niveles de sensibilidad apropiados para el análisis de trazas sin comprometer la resolución de la zona inicial del cromatograma.

Una posible alternativa es la utilización de los dispositivos comerciales denominados inyectores de temperatura programada (PTV). Estos dispositivos permiten modalidades de inyección en las que la temperatura se puede programar, reteniéndose en un primer momento los analitos en el inyector mediante la programación de una temperatura baja y produciéndose posteriormente, con la selección de una rampa rápida de calentamiento del cuerpo del inyector, el paso de los compuestos a la columna cromatográfica en un tiempo breve. Esta modalidad de inyección ha sido muy utilizada en los últimos años en combinación con la técnica de espacio de cabeza.

En cuanto al aumento de la velocidad a la que se realiza la inyección, una solución son los nuevos acoplamientos que existen para esta técnica, consistentes en jeringas termostatadas. Estas jeringas sustituyen a las largas líneas de transferencia de níquel que conectaban el horno de generación de espacio de cabeza y el inyector del cromatógrafo y permiten la inyección rápida del volumen de espacio de cabeza seleccionado.

La generación de espacio de cabeza es ya una técnica de pretratamiento de muestra muy asentada y son innumerables las aplicaciones en las que se propone su utilización, en muchos casos combinada con otras técnicas como la microextracción en fase sólida. Las primeras propuestas se basaron en el análisis de contaminantes en muestras ambientales como los trabajos de 
Pawlinzyn y colaboradores en los que se analizan compuestos orgánicos volátiles $[4,5]$. También se ha propuesto el acoplamiento con técnicas de microextracción en fase líquida para el estudio de contaminantes orgánicos como los trihalometanos en este caso en aguas de consumo [6]. Algunas de las aplicaciones más recientes que acoplan la técnica de espacio de cabeza con técnicas de microextracción en el campo medioambiental se centran en el análisis de contaminantes orgánicos y fármacos en muestras de agua [14-16] y de hidrocarburos policíclicos aromáticos en suelo [17]. En cuanto a la utilización del espacio de cabeza sin acoplamiento a técnicas de microextracción en análisis medioambientales, se han propuesto metodologías para la determinación de ibuprofeno en agua $[18,19]$, para la diferenciación de crudos de petróleo en muestras de suelos contaminados [20] así como para el análisis de restos de gas mostaza en sedimentos marinos in situ mediante el uso de muestreadores portátiles [21].

Esta técnica de pretratamiento ha sido utilizada también en el análisis de muestras alimentarias como miel [22], fruta [23], aceites [24,25], productos cárnicos [26], bizcocho [27], vegetales enlatados [28], alimentos procesados térmicamente [29], zumos [30], salchichas [31] y patatas fritas [32]. En estas tres últimas matrices se ha utilizado la técnica HS-SPME. Se han descrito también aplicaciones para el análisis de surfactantes en muestras textiles [33].

Especial interés se ha prestado recientemente a esta técnica en el campo del bioanálisis donde se aprovechan algunas de sus características. Por un lado, la técnica permite el análisis de compuestos volátiles eliminando interferentes no volátiles de la matriz, en matrices tan complejas como las biológicas (plasma, orina o saliva). Por otro lado, el tratamiento de muestra es 
mínimo y se reduce, muchas veces, a la introducción de la misma en un vial de muestra y su posterior cierre hermético.

Así, se han propuesto métodos que utilizan la técnica de espacio de cabeza acoplada a cromatografía de gases para el análisis de compuestos como aldehídos [34,35], ácidos haloacéticos [36], trihalometanos [37] y compuestos organoestánnicos en muestras de orina $[38,39]$. Este último utiliza la estrategia HS-SPME.

Además, se han llevado a cabo trabajos que utilizan la técnica de espacio de cabeza con microextracción en fase sólida acoplada a técnicas de separación cromatográfica con el objetivo de conocer el contenido de metabolitos volátiles en la orina de individuos sanos y de enfermos y estudiar, de esta manera, las diferencias existentes en algunos compuestos que podrían ser considerados potenciales biomarcadores de diferentes enfermedades [4044]. Algunas aplicaciones, además, incluyen el acoplamiento de la técnica HS con sistemas de inyección de la muestra en frío [45]. Asimismo, se ha utilizado el perfil de compuestos orgánicos volátiles de la orina obtenido mediante la generación de espacio de cabeza, sin utilizar técnicas de microextracción, para discriminar entre individuos sanos y enfermos de tuberculosis [46].

Dentro del campo del bioanálisis existen otras aplicaciones que exploran las posibilidades del acoplamiento directo de esta técnica con un detector de espectrometría de masas para el análisis no separativo de muestras de saliva [47-49], de orina [50,51] y de sangre [51]. También se ha propuesto el uso de esta técnica para el análisis de muestras medioambientales [52,53] y de muestras alimentarias [54-56]. 
En esta memoria se propondrán dos metodologías que utilizan la generación de espacio de cabeza estático como técnica de pretratamiento de muestra para la determinación en muestras de orina de aminoácidos (II.1) y de posibles biomarcadores (II.2).

\section{Bibliografía}

[1] B. Kolb, L.S. Ettre, Static Headspace-Gas Chromatography, Wiley Interstice, Hoboken, New Jersey, 2006.

[2] A.C. Soria, M.J. García-Sarrió, M.L. Sanz, Volatile sampling by headspace techniques, Trends Anal. Chem. 71 (2015) 85-99.

[3] R.P. Belardi, J. Pawliszyn, Application of chemically modified fused silica fibers in the extraction of organics from water matrix samples and their rapid transfer to capillary columns, Water Pollut. Res. J. Can. 24 (1989) 179191.

[4] C.L. Arthur, J. Pawliszyn, Solid phase microextraction with thermal desorption using fused silica optical fibers, Anal. Chem. 62 (1990) 2145-2148.

[5] Z. Zhang, J. Pawliszyn, Headspace solid-phase microextraction, Anal. Chem. 65 (1993) 1843-1852.

[6] R-s. Zhao, W-j. Lao, X-b. Xu, Headspace liquid-phase microextraction of trihalomethanes in drinking water and their gas chromatographic determination, Talanta 62 (2004) 751-756. 
[7] J.W. Swinnerton, V.J. Linnenboom, C.H. Cheek, Determination of dissolved gases in aqueous solutions by gas chromatography, Anal. Chem. 34 (1962) 483-485.

[8] J.W. Swinnerton, V.J. Linnenboom, C.H. Cheek, Revised sampling procedure for determination of dissolved gases in solution by gas chromatography, Anal. Chem. 34 (1962) 1509.

[9] T.A. Bellar, J.J. Lichtenberg, Determining volatile organics at microgram-per-litre levels by gas chromatography, J. Am. Water Works Ass. 66 (1974) 739-744.

[10] B. Kolb, Headspace sampling with capillary columns, J. Chromatogr. A 842 (1999) 163-205.

[11] H-J. Stan, M. Linkerhägner, Large-volume injection in residue analysis with capillary gas chromatography using a conventional autosampler and injection by programmed-temperature vaporization with solvent venting, J. Chromatogr. A 727 (1996) 275-289.

[12] J. Zrostlíková, J. Hajšlová, M. Godula, K. Maštovská, Performance of programmed temperature vaporizer, pulsed splitless and on-column injection techniques in analysis of pesticide residues in plant matrices, J. Chromatogr. A 937 (2001) 73-86.

[13] B. Kolahgar, E. Pfannkock, Technical Note 36, Gerstel, Mülheim/Ruhr. [14] C. Martínez, N. Ramírez, V. Gómez, E. Pocurull, F. Borrull, Simultaneous determination of 76 micropollutants in water samples by headspace solid phase microextraction and gas chromatography-mass 
spectrometry, Talanta 116 (2013) 937-945.

[15] P-S. Chen, Y-H. Tseng, Y-L. Chuang, J-H. Chen, Determination of volatile organic compounds in water using headspace knotted hollow fiber microextraction, J. Chromatogr. A 1395 (2015) 41-47.

[16] M. Behzadi, M. Mirzaei, Poly(o-anisidine)/graphene oxide nanosheets composite as coating for the headspace solid-phase microextraction of benzene, toluene, ethylbenzene and xylenes, J. Chromatogr. A 1443 (2016) $35-42$.

[17] A.R. Ghiasvand, M. Pirdadeh-Beiranvand, Cooling/heating-assisted headspace solid-phase microextraction of polycyclic aromatic hydrocarbons from contaminated soils, Anal. Chim. Acta 900 (2015) 56-66.

[18] J.L. Pérez Pavón, A.M. Casas Ferreira, M.E. Fernández Laespada, B. Moreno Cordero, In situ derivatization reaction and determination of ibuprofen in water samples using headspace generation-programmed temperature vaporization-gas chromatography-mass spectrometry, J. Chromatogr. A 1216 (2009) 6728-6734.

[19] A.M. Casas Ferreira, M.E. Fernández Laespada, J.L. Pérez Pavón, B. Moreno Cordero, Headspace sampling with in situ carbodiimide-mediated derivatization for the determination of ibuprofen in water samples, J. Chromatogr. A 1218 (2011) 4856-4862.

[20] J.L. Pérez Pavón, A. Guerrero Peña, C. García Pinto, B. Moreno Cordero, Differentiation of types of crude oils in polluted soil samples by headspace-fast gas chromatography-mass spectrometry, J. Chromatogr. A 1137 (2006) 101-109. 
[21] R. Magnusson, T. Nordlander, A. Östin, Development of a dynamic headspace gas chromatography-mass spectrometry method for on-site analysis of sulfur mustard degradation products in sediments, J. Chromatogr. A 1429 (2016) 40-52.

[22] L.A. Frink, D.W. Armstrong, The utilization of two detectors for the determination of water in honey using headspace gas chromatography, Food Chem. 205 (2016) 23-27.

[23] C-Y. Zhang, Q. Zhang, C-H. Zhong, M-Q. Guo, Analysis of volatile compounds responsible for kiwifruit aroma by desiccated headspace gas chromatography-mass spectrometry, J. Chromatogr. A 1440 (2016) 255-259. [24] W. Hu, L. Zhang, P. Li, X. Wang, Q. Zhang, B. Xu, X. Sun, F. Ma, X. Ding, Characterization of volatile components in four vegetable oils by headspace two-dimensional comprehensive chromatography time-of-flight mass spectrometry, Talanta 129 (2014) 629-635.

[25] J.L. Pérez Pavón, M. del Nogal Sánchez, M.E. Fernández Laespada, B. Moreno Cordero, Determination of filbertone in spiked olive oil samples using headspace-programmed temperature vaporization-gas chromatography-mass spectrometry, Anal. Bioanal. Chem. 394 (2009) 1463-1470.

[26] R. Bosse, M. Wirth, A. Konstanz, T. Becker, J. Weiss, M. Gibis, Determination of volatile marker compounds in raw ham using headspacetrap gas chromatography, Food Chem. 219 (2017) 249-259.

[27] M. Cepeda-Vázquez, D. Blumenthal, V. Camel, B. Rega, Multivariate optimization of headspace trap for furan and furfural simultaneous determination in sponge cake, Talanta 164 (2017) 708-715. 
[28] M.J. Cardador, M. Gallego, Static headspace-gas chromatography-mass spectrometry for the simultaneous determination of trihalomethanes and haloacetic acids in canned vegetables, J. Chromatogr. A 1454 (2016) 9-14.

[29] M. Shen, Q. Liu, H. Jia, Y. Jiang, S. Nie, J. Xie, C. Li, M. Xie, Simultaneous determination of furan and 2-alkylfurans in heat-processed foods by automated static headspace gas chromatography-mass spectrometry, LWT-Food Sci. and Technol. 72 (2016) 44-54.

[30] G. Hu, Y. Zhu, M. Hernández, T. Koutchma, S. Shao, An efficient method for the simultaneous determination of furan, 2-methylfuran and 2pentylfuran in fruit juices by headspace solid phase microextraction and gas chromatography-flame ionisation detector, Food Chem. 192 (2016) 9-14.

[31] E. Canellas, P. Vera, C. Nerín, Multiple headspace-solid phase microextraction for the determination of migrants coming from a self-stick label in fresh sausage, Food Chem. 197 (2016) 24-29.

[32] A.R. Ghiasvand, S. Hajipour, Direct determination of acrylamide in potato chips by using headspace solid-phase microextraction coupled with gas chromatography-flame ionization detection, Talanta 146 (2016) 417-422.

[33] S-X. Zhang, X-S. Chai, B-X. Huang, X-X. Mai, A robust method for determining water-extractable alkylphenol polyethoxylates in textile products by reaction-based headspace gas chromatography, J. Chromatogr. A 1406 (2015) 94-98.

[34] M. Serrano, M. Gallego, M. Silva, Analysis of endogenous aldehydes in human urine by static headspace gas chromatography-mass spectrometry, J. Chromatogr. A 1437 (2016) 241-246. 
[35] A. Pérez Antón, A.M. Casas Ferreira, C. García Pinto, B. Moreno Cordero, J.L. Pérez Pavón, Headspace generation coupled to gas chromatography-mass spectrometry for the automated determination and quantification of endogenous compounds in urine. Aldehydes as possible markers of oxidative stress, J. Chromatogr. A 1367 (2014) 9-15.

[36] M.J. Cardador, M. Gallego, Determination of haloacetic acids in human urine by headspace gas chromatography-mass spectrometry, J. Chromatogr. B 878 (2010) 1824-1830.

[37] J. Caro, A. Serrano, M. Gallego, Sensitive headspace gas chromatography-mass spectrometry determination of trihalomethanes in urine, J. Chromatogr. B 848 (2007) 277-282.

[38] A. Valenzuela, G. Lespes, W. Quiroz, L.F. Aguilar, M.A. Bravo, Speciation analysis of organotin compounds in human urine by headspace solid-phase micro-extraction and gas chromatography with pulsed flame photometric detection, Talanta 125 (2014) 196-203.

[39] Z. Cui, K. Zhang, Q. Zhou, J. Liu, G. Jiang, Determination of methyltin compounds in urine of occupationally exposed and general population by in situ ethylation and headspace SPME coupled with GC-FPD, Talanta 85 (2011) 1028-1033.

[40] C.L. Silva, M. Passos, J.S. Câmara, Investigation of urinary volatile organic metabolites as potential cancer biomarkers by solid-phase microextraction in combination with gas chromatography-mass spectrometry, Br. J. Cancer 105 (2011) 1894-1904.

[41] Y. Hanai, K. Shimono, K. Matsumura, A. Vachani, S. Albelda, K. 
Yamazaki, G.K. Beauchamp, H. Oka, Urinary Volatile Compounds as Biomarkers for Lung Cancer, Biosci. Biotechnol. Biochem. 76 (2012) 679-684. [42] S.M. Rocha, M. Caldeira, J. Carrola, M. Santos, N. Cruz, I.F. Duarte, Exploring the human urine metabolomic potentialities by comprehensive two-dimensional gas chromatography coupled to time of flight mass spectrometry, J. Chromatogr. A 1252 (2012) 155-163.

[43] S. Smith, H. Burden, R. Persad, K. Whittington, B.L. Costello, N.M. Ratcliffe, C.S. Probert, A comparative study of the analysis of human urine headspace using gas chromatography-mass spectrometry, J. Breath Res. 2 (2008) 037022 (10pp). doi:10.1088/1752-7155/2/3/037022.

[44] M.S. Monteiro, M. Carvalho, M.L. Bastos, P.G. Pinho, Chapter 12: Potentiality of volatiles to discriminate patients with cancer by using chemometric tools. En el libro: Identification and Data processing methods in metabolomics, Publisher: Future Science, Editors: Eds. London: Future Science, 2015. pp. 167-184.

[45] H.G. Wahl, A. Hoffmann, D. Luft, H.M. Liebich, Analysis of volatile organic compounds in human urine by headspace gas chromatography-mass spectrometry with a multipurpose sampler, J. Chromatogr. A 847 (1999) 117125.

[46] K.M. Banday, K.K. Pasikanti, E.C.Y. Chan, R. Singla, K.V.S. Rao, V.S. Chauhan, R.K. Nanda, Use of urine volatile organic compounds to discriminate tuberculosis patients from healthy subjects, Anal. Chem. 83 (2011) 5526-5534.

[47] M. del Nogal Sánchez, E. Hernández García, J.L. Pérez Pavón, B. 
Moreno Cordero, Fast analytical methodology based on mass spectrometry for the determination of volatile biomarkers in saliva, Anal. Chem. 84 (2012) 379385.

[48] M. del Nogal Sánchez, P.Á. Callejo Gómez, J.L. Pérez Pavón, B. Moreno Cordero, Á.P. Crisolino Pozas, Á. Sánchez Rodríguez, Sensitivity enhancement in the determination of volatile biomarkers in saliva using a mass spectrometry-based electronic nose with a programmed temperature vaporizer, Anal. Chem. 86 (2014) 7890-7898.

[49] A. Pérez Antón, M. del Nogal Sánchez, Á.P. Crisolino Pozas, J.L. Pérez Pavón, B. Moreno Cordero, Headspace-programmed temperature vaporizermass spectrometry and pattern recognition techniques for the analysis of volatiles in saliva samples, Talanta 160 (2016) 21-27.

[50] A. Pérez Antón, Á. García Ramos, M. del Nogal Sánchez, J.L. Pérez Pavón, B. Moreno Cordero, Á.P. Crisolino Pozas, Headspace-programmed temperature vaporization-mass spectrometry for the rapid determination of possible volatile biomarkers of lung cancer in urine, Anal. Bioanal. Chem. 408 (2016) 5239-5246.

[51] J.W.T. Yates, M.J. Chappell, J.W. Gardner, C.S. Dow, C. Dowson, A. Hamood, F. Bolt, L. Beeby, Data reduction in headspace analysis of blood and urine samples for robust bacterial identification, Comput. Methods Programs Biomed. 79 (2005) 259-271.

[52] M. del Nogal Sánchez, J.L. Pérez Pavón, M.E. Fernández Laespada, C. García Pinto, B. Moreno Cordero, Factors affecting signal intensity in headspace mass spectrometry for the determination of hydrocarbon pollution 
in beach sands, Anal. Bioanal. Chem. (2005) 372-380.

[53] A. Serrano, M. Gallego, Rapid determination of total trihalomethanes index in drinking water, J. Chromatogr. A 1154 (2007) 26-33.

[54] C. Pérès, F. Begnaud, L. Eveleigh, J-L. Berdagué, Fast characterization of foodstuff by headspace mass spectrometry (HS-MS), Trends Anal. Chem. 22 (2003) 858-866.

[55] F. Pena, S. Cardenas, M. Gallego, M. Valcárcel, Direct olive oil authentication: detection of adulteration of olive oil with hazelnut oil by direct coupling of headspace and mass spectrometry, and multivariate regression techniques, J. Chromatogr. A 1074 (2005) 215-221.

[56] D. Cozzolino, H.E. Smyth, W. Cynkar, L. Janik, R.G. Dambergs, M. Gishen, Use of direct headspace-mass spectrometry coupled with chemometrics to predict aroma properties in Australian Riesling wine, Anal. Chim. Acta 621 (2008) 2-7. 



\section{3 \\ LÍQUIDOS IÓNICOS EN CROMATOGRAFÍA DE GASES}



La investigación con líquidos iónicos es una de las áreas de estudio de mayor crecimiento en los últimos años. El amplio número de usos y aplicaciones que se les pueden dar ha hecho que se les haya encontrado utilidad en campos muy diferentes dentro de la química y otras ciencias.

El término "líquidos iónicos" hace referencia a un tipo de sales orgánicas que tienen un bajo punto de fusión. Gracias a esta propiedad se mantienen líquidas en un amplio intervalo de temperaturas. Su composición química consiste en cationes orgánicos asociados con aniones que pueden ser inorgánicos u orgánicos. Los cationes orgánicos más habituales en este tipo de compuestos contienen nitrógeno o fósforo [1]. Algunos de los cationes más utilizados se muestran a continuación (figura 3.1):
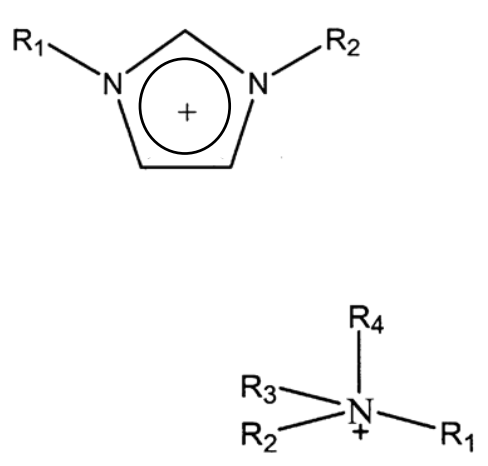
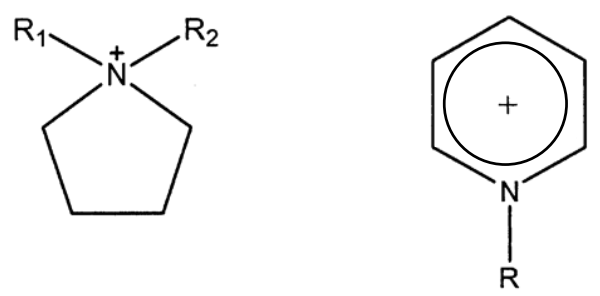

Figura 3.1. Cationes más comúnmente utilizados en la síntesis de líquidos

$$
\text { iónicos [1] }
$$

Las cadenas alquílicas (R) más usadas son: metil, etil, propil, butil, hexil, octil y decil. Los aniones (figura 3.2) pueden ser inorgánicos, como cloruro $\left(\mathrm{Cl}^{-}\right)$, tetrafluoroborato $\left(\mathrm{BF}_{4}^{-}\right)$y hexafluorofosfato $\left(\mathrm{PF}_{6}^{-}\right)$, pero 
normalmente consisten en aniones orgánicos como trifluoroetanoato $\left[\mathrm{CF}_{3} \mathrm{CO}_{2}\right]^{-}, \quad$ trifluorometilsulfonato $\quad\left[\mathrm{CF}_{3} \mathrm{SO}_{3}\right]^{-} \quad$ y bis[(trifluorometil)sulfonil]imida $\left[\mathrm{N}\left(\mathrm{CF}_{3} \mathrm{SO}_{2}\right)_{2}\right]^{-}[2]$.

$\mathrm{Cl}^{-}$<smiles>O=C([O-])C(F)(F)F</smiles>

$\left[\mathrm{CF}_{3} \mathrm{CO}_{2}\right]^{-}$
$\mathrm{BF}_{4}^{-}$<smiles>O=S(=O)([O-])C(F)(F)F</smiles>

$\left[\mathrm{CF}_{3} \mathrm{SO}_{3}\right]^{-}$
$\mathrm{PF}_{6}^{-}$<smiles>O=S(=O)([18O])NS(=O)(=O)[18F]</smiles>

$\left[\mathrm{N}\left(\mathrm{CF}_{3} \mathrm{SO}_{2}\right)_{2}\right]^{-}$

Figura 3.2. Aniones más comúnmente utilizados para la síntesis de líquidos

$$
\text { iónicos [1] }
$$

Estos líquidos iónicos presentan características especiales [2] tales como alta estabilidad frente a la temperatura (siendo estables hasta los $300^{\circ} \mathrm{C}$ ), baja volatilidad, capacidad para conducir la electricidad, amplio rango de viscosidades y capacidad de solubilizar una gran variedad de moléculas. Todas estas propiedades, junto con el hecho de que pueden ser sintetizados de manera que sean inmiscibles tanto con agua como con disolventes orgánicos no polares, les confieren numerosas utilidades en campos muy diferentes dentro de la química. Concretamente, en química analítica los líquidos iónicos pueden utilizarse como disolventes de extracción en técnicas de preparación de muestra, como aditivos para las fases móviles en cromatografía de líquidos, como modificadores de la pared de los capilares de electroforesis y como sensores. En el campo de la cromatografía destaca su utilización como fases estacionarias tanto en columnas de cromatografía líquida, como en columnas capilares de cromatografía de gases. 
La investigación en el uso de sales fundidas como fases estacionarias en cromatografía de gases (GC) comenzó en los años 50. Barber y colaboradores [3] prepararon fases estacionarias utilizando estearatos fundidos de $\mathrm{Mn}, \mathrm{Co}, \mathrm{Ni}$, Cu y Zn para separar alcoholes y aminas. Poole y colaboradores $[4,5]$ prepararon y probaron fases estacionarias de nitrato de etilamonio y bromuro de etilpiridinio. Éstas actúan como fases estacionarias polares y sus interacciones dipolares o de enlaces por puentes de hidrógeno contribuyen significativamente a la retención. Sin embargo, estas primeras columnas presentaban todavía algunos inconvenientes, como una baja eficiencia y una pobre estabilidad térmica.

Las primeras fases estacionarias de líquidos iónicos con las que se obtuvieron buenos resultados fueron las de imidazolio [6]. Concretamente, se utilizaron sales de hexafluorofosfato y cloruro de 1-butil-3-metilimidazolio ([BMIM] $\left[\mathrm{PF}_{6}\right]$ y $\left.[\mathrm{BMIM}][\mathrm{Cl}]\right)$ para recubrir las paredes de columnas capilares de sílice. Las propiedades de estas fases estacionarias se examinaron por medio de cromatografía en fase inversa con un gran número de moléculas prueba. Estas columnas muestran un doble comportamiento ya que retienen tanto compuestos polares como no polares [7].

En 2005, se consiguieron mediante entrecruzamiento (cross-linking) fases estacionarias de alta estabilidad y selectividad utilizando una pequeña cantidad de radicales libres como iniciadores [8]. Más recientemente se han desarrollado nuevas fases estacionarias de alta estabilidad que permanecen estables hasta $350{ }^{\circ} \mathrm{C}[9,10]$.

Una de las características que hace que los líquidos iónicos sean buenas fases estacionarias es el amplio rango de temperaturas en el que se mantienen 
líquidos, permitiendo aumentar el margen de temperaturas de trabajo. Además, estos compuestos son poco volátiles y, por consiguiente, las columnas con fases estacionarias de líquidos iónicos tienen un menor sangrado y una vida útil más larga. La ausencia de grupos hidroxilo las hace más estables ante la humedad y el oxígeno. La posibilidad de sintetizarlas con distintos grupos catiónicos y aniónicos, así como con distintas longitudes de cadena permite dotarlas de una selectividad "a la carta".

La separación que se consigue con estas fases estacionarias depende, en gran medida, de la naturaleza de los cationes y los aniones de los líquidos iónicos. A partir de los estudios realizados se ha llegado a la conclusión de que los líquidos iónicos monocatiónicos no tienen propiedades cromatográficas tan buenas como los policatiónicos, siendo los más utilizados los dicatiónicos.

En cuanto a la estructura de estas sales dicatiónicas, los cationes se encuentran unidos por medio de una cadena carbonada de longitud variable y asociados a estos cationes, los correspondientes aniones.

Teniendo en cuenta todo esto, a partir de los trabajos realizados por el profesor Daniel W. Armstrong de la Universidad de Texas en Arlington, EE. UU. [11], la casa comercial Supelco (Bellefonte, PA, USA) lanzó al mercado las primeras fases estacionarias de líquidos iónicos, que se encuentran desde entonces comercialmente disponibles y que son objeto de estudio en el primero de los trabajos que se describen en esta memoria (I.1).

Con algunas de estas columnas comerciales con fases estacionarias de líquidos iónicos se ha propuesto la determinación de nitrosaminas y metabolitos de la cafeína en aguas de desecho eligiéndose como óptima para la 
separación, en el caso de las primeras, la columna SLB-IL111 basada en el líquido iónico bis(trifluorometilsulfonil)imidato de 1,5-di(2,3dimetilimidazolio)pentano y en el caso de los segundos compuestos, la columna SLB-IL59 a base de bis(trifluorometilsulfonil)imidato de 1,12di(tripropilfosfonio)dodecano [12]. En esta misma matriz se ha estudiado la determinación de benzotiazoles y benzotriazoles con cinco fases estacionarias de líquidos iónicos mejorándose su separación, que resulta muy problemática utilizando columnas convencionales, y siendo de nuevo la columna SLB-IL59 la que mejores resultados proporciona [13]. Para el análisis de policlorobifenilos (PCBs) y pesticidas organoclorados se propuso la utilización de una columna SLB-IL111 [14]. Esta columna, además, fue la que mejores resultados proporcionó entre cinco columnas examinadas en otro trabajo en el que se estudiaban las posibilidades que ofrecía el uso de este tipo de fases estacionarias en cromatografía multidimensional (GCxGC). Los líquidos iónicos alteran el orden de elución de algunos compuestos y resultan adecuados para su uso en esta modalidad de cromatografía de gases como columna secundaria [15-17].

Se ha propuesto la utilización de estas columnas para el estudio de haloanisoles en vinos [18]; en este trabajo se estudiaron cuatro columnas entre las que finalmente se seleccionó la columna SLB-IL59 como óptima para la separación de estos compuestos.

No obstante, a pesar de la existencia de columnas comercialmente disponibles con diferentes fases estacionarias de líquidos iónicos, son muchos los trabajos que se han realizado en los últimos años en los que se proponen nuevos líquidos iónicos como fases estacionarias de cromatografía de gases 
$[19,20]$. Así, recientemente se han propuesto una serie de fases estacionarias basadas en guanidinio [21,22]. Otro grupo de líquidos iónicos que ha recibido creciente interés es el de los basados en aminoácidos (AAILs) ya que se han propuesto como compuestos más biodegradables y biocompatibles que los líquidos iónicos convencionales y se han descrito en aplicaciones como el análisis de ésteres metílicos de ácidos grasos (FAMEs) en grasa láctea mediante una fase estacionaria con un líquido iónico monocatiónico de imidazolio derivado de la L-fenilalanina [23].

Muchos trabajos, además, se centran en la caracterización de estas nuevas fases y de las interacciones que presentan estudiando la constante de McReynolds y el modelo de solvatación de Abraham [21,23].

Algunas de estas nuevas fases estacionarias, además, han sido propuestas con el objetivo de mejorar algunas de las características de las comercialmente disponibles y así alargar su vida útil haciéndolas más estables $[15,16]$.

\section{Bibliografía}

[1] P. Sun, D.W. Armstrong, Ionic liquids in analytical chemistry, Anal. Chim. Acta 661 (2010) 1-16.

[2] J.S. Wilkes, Properties of ionic liquid solvents for catalysis, J. Mol. Catal. A: Chem. 214 (2004) 11-17.

[3] D.W. Barber, C.S.G. Phillips, G.F. Tusa, A. Verdin, The 
chromatography of gases and vapours. Part VI.* Use of the stearates of bivalent manganese, cobalt, nickel, copper, and zinc as column liquids in gas chromatography, J. Chem. Soc. (1959) 18-24.

[4] F. Pacholec, H.T. Butler, C.F. Poole, Molten organic salt phase for gasliquid chromatography, Anal. Chem. 54 (1982) 1938-1941.

[5] F. Pacholec, C.F. Poole, Stationary phase properties of the organic molten salt ethylpyridinium bromide in gas chromatography, Chromatographia 17 (1983) 370-374.

[6] D.W. Armstrong, L. He, Y.S. Liu, Examination of ionic liquids and their interaction with molecules, when used as stationary phases in gas chromatography, Anal. Chem. 71 (1999) 3873-3876.

[7] J.L. Anderson, D.W. Armstrong, High-stability ionic liquids. A new class of stationary phases for gas chromatography, Anal. Chem. 75 (2003) 4851-4858.

[8] J.L. Anderson, D.W. Armstrong, Immobilized ionic liquids as highselectivity/high-temperature/high-stability gas chromatography stationary phases, Anal. Chem. 77 (2005) 6453-6462.

[9] K. Huang, X. Han, X. Zang, D.W. Armstrong, PEG-linked geminal dicationic ionic liquids as selective, high-stability gas chromatographic stationary phases, Anal. Bioanal. Chem. 389 (2007) 2265-2275.

[10] Z.S. Breitbach, D.W. Armstrong, Characterization of phosphonium ionic liquids through a linear solvation energy relationship and their use as GLC stationary phases, Anal. Bioanal. Chem. 390 (2008) 1605-1617. 
[11] L.M. Sidisky and M.D. Buchanan, Supelco Patented Ionic Liquid GC Phase Technology, Supelco Reporter 26 (2008) 3.

[12] C. Reyes-Contreras, C. Domínguez, J.M. Bayona, Determination of nitrosamines and caffeine metabolites in wastewater using gas chromatography mass spectrometry and ionic liquid stationary phases, J. Chromatogr. A 1261 (2012) 164-170.

[13] C. Domínguez, C. Reyes-Contreras, J.M. Bayona, Determination of benzothiazoles and benzotriazoles by using ionic liquid stationary phases in gas chromatography mass spectrometry. Application to their characterization in wastewater, J. Chromatogr. A 1230 (2012) 117-122.

[14] J. de Boer, D. Blok, A. Ballesteros-Gómez, Assessment of ionic liquid stationary phases for the determination of polychlorinated biphenyls, organochlorine pesticides and polybrominated diphenyl ethers, J. Chromatogr. A 1348 (2014) 158-163.

[15] C. Zhang, R.A. Park, J.L. Anderson, Crosslinked structurally-tuned polymeric ionic liquids as stationary phases for the analysis of hydrocarbons in kerosene and diesel fuels by comprehensive two-dimensional gas chromatography, J. Chromatogr. A 1440 (2016) 160-171.

[16] C. Zhang, I.C. Ingram, L.W. Hantao, J.L. Anderson, Identifying important structural features of ionic liquid stationary phases for the selective separation of nonpolar analytes by comprehensive two-dimensional gas chromatography, J. Chromatogr. A 1386 (2015) 89-97.

[17] H. Han, C. Zhang, R.A. O’Brien, A. Benchea, J.H. Davis Jr., J.L. Anderson, Lipidic ionic liquid stationary phases for the separation of aliphatic 
hydrocarbons by comprehensive two-dimensional gas chromatography, J. Chromatogr. A 1481 (2017) 127-136.

[18] C. García Pinto, A. Pérez Antón, J.L. Pérez Pavón, B. Moreno Cordero, Coupling of microextraction by packed sorbents with gas chromatography with ionic liquid stationary phases for the determination of haloanisoles in wines, J. Chromatogr. A 1260 (2012) 200-205.

[19] C.F. Poole, N. Lenca, Gas chromatography on wall-coated open-tubular columns with ionic liquid stationary phases, J. Chromatogr. A 1357 (2014) 87109.

[20] C. Ragonese, D. Sciarrone, P.Q. Tranchida, P. Dugo, L. Mondello, Use of ionic liquids as stationary phases in hyphenated gas chromatography techniques, J. Chromatogr. A 1255 (2012) 130-144.

[21] L. Qiao, K. Lu, M. Qi, R. Fu, Separation performance of guanidiniumbased ionic liquids as stationary phases for gas chromatography, J. Chromatogr. A 1276 (2013) 112-119.

[22] L. Wang, X. Wang, M. Qi, R. Fu, Cucurbit[6]uril in combination with guanidinium ionic liquid as a new type of stationary phase for capillary gas chromatography, J. Chromatogr. A 1334 (2014) 112-117.

[23] L. González Mendoza, J. González-Álvarez, C. Fernández Gonzalo, P. Arias-Abrodo, B. Altava, S.V. Luis, M.I. Burguete, M.D. Gutiérrez-Álvarez, Gas chromatographic analysis of fatty acid methyl esters of milk fat by an ionic liquid derived from L-phenylalanine as the stationary phase, Talanta 143 (2015) 212-218. 




\subsection{Esquema de las configuraciones instrumentales utilizadas}

Para el desarrollo de los diferentes métodos analíticos que se han propuesto en este trabajo se han utilizado dos configuraciones instrumentales:

- Un sistema de introducción de muestras líquidas, un cromatógrafo de gases (GC) con columnas capilares de líquidos iónicos equipado con un inyector de temperatura programada (PTV) y un micro-detector de captura electrónica ( $\mu$-ECD). Esta configuración, mostrada en la figura 4.1, fue la utilizada para la primera metodología del capítulo I de esta memoria (I.1).

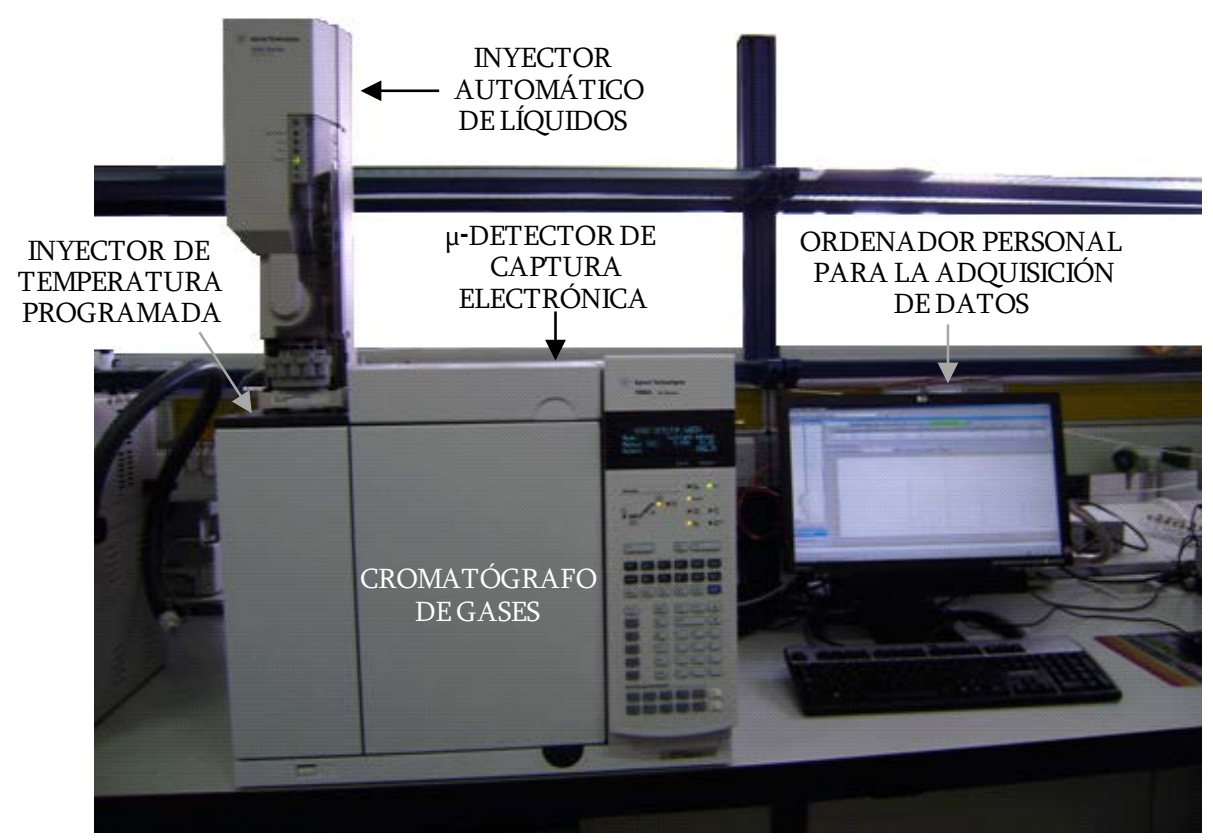

Figura 4.1. Configuración instrumental PTV-GC- $\mu E C D$ 
- Un automuestrador MPS2 con módulo para microextracción con sorbentes empaquetados (MEPS) y para generación de espacio de cabeza (HS) acoplado a un cromatógrafo de gases (GC) con inyector de temperatura programada (PTV) y un espectrómetro de masas de tipo cuadrupolo (q-MS). En la figura 4.2 se muestra la fotografía de esta configuración utilizada para la segunda metodología del capítulo I (I.2) y para las dos metodologías del capítulo II (II.1 y II.2).

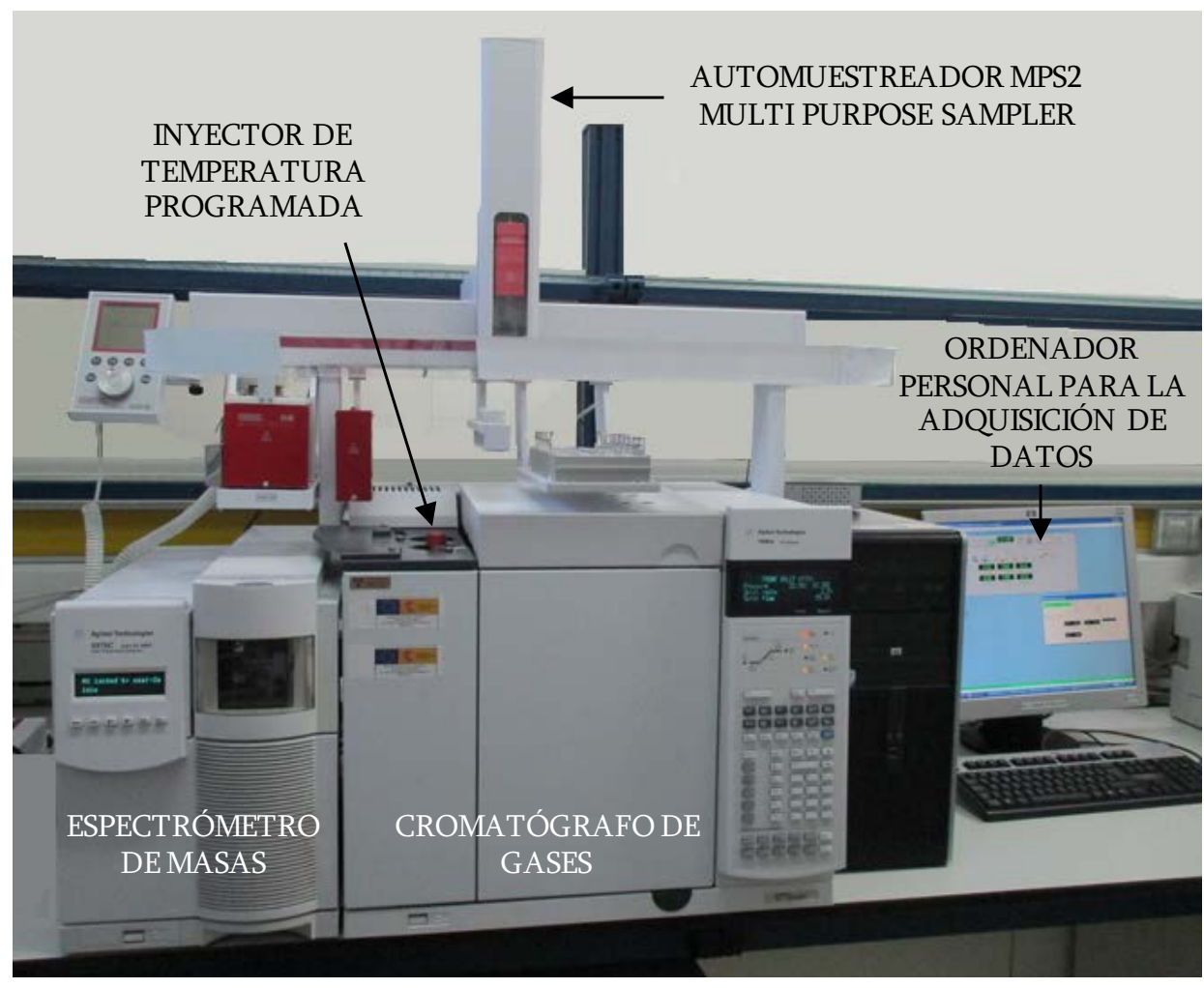

Figura 4.2. Configuración instrumental MEPS o HS acoplado a PTV-GC-MS 


\subsection{Sistema de introducción de muestras-PTV-GC- $\mu E C D$}

\subsubsection{Sistema de introducción de muestras}

El modelo utilizado es un inyector automático de líquidos Agilent 7683 (Agilent Technologies, Waldbronn, Alemania). La torre del inyector contiene una jeringa que permite la inyección de muestras líquidas. La jeringa utilizada tiene un volumen de $10 \mu \mathrm{L}$ de los que permite inyectar en el sistema cromatográfico un máximo de $5 \mu \mathrm{L}$.

\subsubsection{Inyector de temperatura programada}

La muestra es transferida al sistema cromatográfico por medio de un vaporizador de temperatura programada (PTV) Agilent Technologies 6890 (CIS-4). Un esquema del dispositivo utilizado se muestra en la figura 4.3.

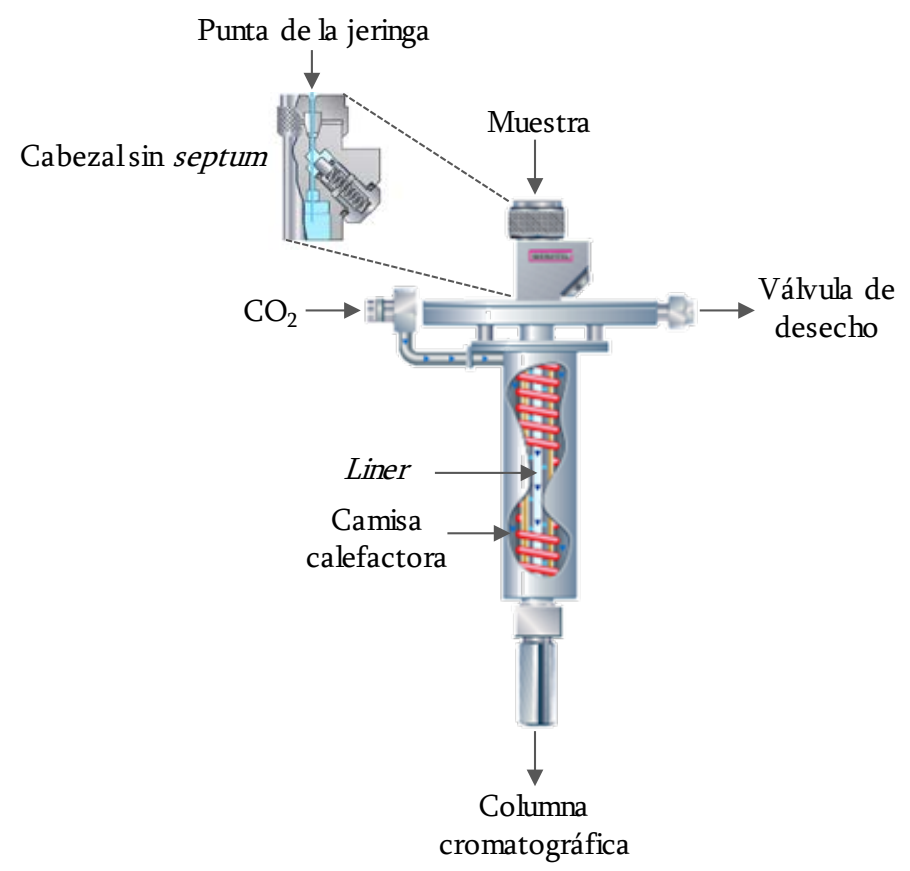

Figura 4.3. Esquema de un inyector PTV 
El cabezal del inyector utilizado no tiene septum, sino que cuenta con una válvula de sellado que se desplaza durante la inyección para permitir el paso de la jeringa. El enfriamiento se logra mediante $\mathrm{CO}_{2}$ líquido, que permite alcanzar la temperatura de $-78{ }^{\circ} \mathrm{C}$, mientras que el calentamiento se consigue mediante una camisa calefactora que proporciona un aumento lineal y homogéneo de la temperatura en el cuerpo del inyector y permite seleccionar rampas de calentamiento desde $120^{\circ} \mathrm{C} \mathrm{min}{ }^{-1}$ hasta $720{ }^{\circ} \mathrm{C} \mathrm{min}-1$ así como dos rampas de temperatura consecutivas.

El PTV está equipado con una cámara de vaporización (liner) rellena de Tenax-TA ${ }^{\circledR}$ de dimensiones $71 \mathrm{~mm}$ x $2.0 \mathrm{~mm}$ y un volumen interno de 180 $\mu \mathrm{L}$. Este material de relleno es un polímero poroso de óxido de 2,6-difenileno con propiedades hidrofóbicas diseñado para retener compuestos orgánicos volátiles y semivolátiles especialmente en muestras con alto contenido de humedad. El gas portador es helio N50 (pureza de 99.999\%, Air Liquide).

\subsubsection{Cromatógrafo de gases}

El análisis cromatográfico se lleva a cabo mediante un cromatógrafo de gases de Agilent Technologies modelo 7890A. El horno del cromatógrafo permite programar hasta cinco rampas de temperatura. Las rampas máximas permitidas por el equipo son $120^{\circ} \mathrm{C} \mathrm{min}{ }^{-1}$ hasta $70{ }^{\circ} \mathrm{C}, 95^{\circ} \mathrm{C} \mathrm{min}{ }^{-1}$ de 70 a 115 ${ }^{\circ} \mathrm{C}, 65{ }^{\circ} \mathrm{C} \min ^{-1}$ de 115 a $175{ }^{\circ} \mathrm{C}, 45^{\circ} \mathrm{C} \min ^{-1}$ de 175 a $300{ }^{\circ} \mathrm{C}$ y $35^{\circ} \mathrm{C}$ min $^{-1}$ de 300 a $400{ }^{\circ} \mathrm{C}$. El gas portador utilizado es helio N50 (pureza de 99.999\%; Air Liquide).

Las columnas utilizadas con esta configuración instrumental fueron fases estacionarias de líquidos iónicos suministradas por la casa Supelco a 
través de Sigma-Aldrich (Steinheim, Alemania) dentro del proyecto SILEP. En concreto, se evaluaron cuatro columnas capilares de sílice fundida de $30 \mathrm{~m}$ de longitud con un diámetro interno de $0.25 \mathrm{~mm}$ y un espesor de fase estacionaria $0.2 \mu \mathrm{m}$ (figura 4.4) y una columna convencional HP5 (30 m x $0.32 \mathrm{~mm}$ x 0.25 $\mu \mathrm{m})$ de Agilent Technologies.

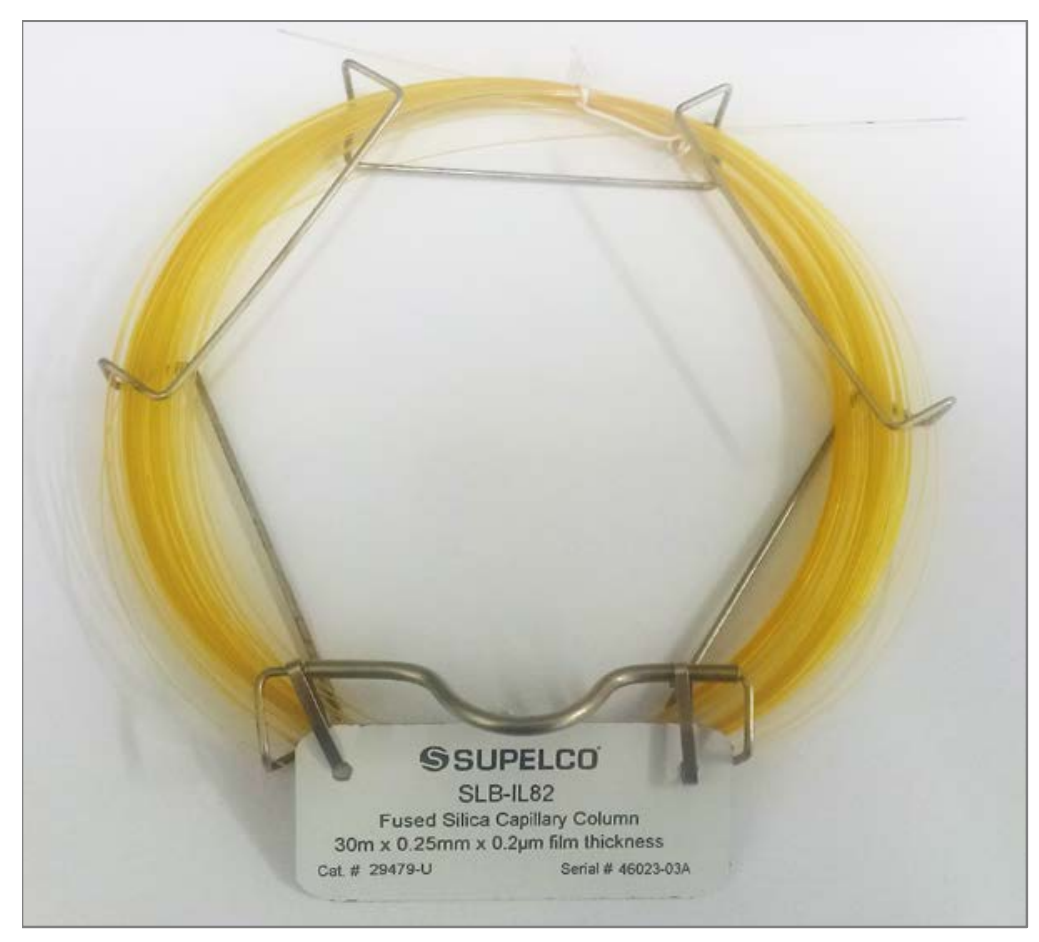

Figura 4.4. Columna capilar SLB-IL82

Los líquidos iónicos que constituyen cada una de estas cuatro columnas utilizadas con fases no ligadas se muestran en la Tabla 4.1. 
Tabla 4.1. Líquido iónico de la fase estacionaria de cada columna evaluada

SLB-IL59




\subsubsection{Micro-detector de captura electrónica}

El sistema cromatográfico utilizado está equipado con un microdetector de captura electrónica ( $\mu$-ECD) de Agilent Technologies (Waldbronn, Alemania) con una fuente de radiación de ${ }^{63} \mathrm{Ni}$. La temperatura máxima permitida por la fuente de radiación es de $400{ }^{\circ} \mathrm{C}$. El volumen de la zona de detección es diez veces menor que el de cualquier otro detector de captura electrónica convencional (ECD) y la velocidad de paso de los analitos a través de la zona de detección es superior, lo que reduce el tiempo de residencia de los analitos en la célula. Otro aspecto novedoso del detector $\mu$-ECD es la disposición del ánodo (ánodo escondido), que está situado de tal manera que la probabilidad de que los contaminantes lleguen hasta él es mínima. Estas características del detector se traducen en una mayor sensibilidad a la vez que disminuyen las posibilidades de contaminación de la celda. Para facilitar el transporte de la muestra a través de la célula del detector se utiliza una corriente de gas auxiliar que se une a la corriente procedente de la columna. El flujo de este gas afecta a la sensibilidad del detector y a la resolución del cromatograma. Los valores habituales de flujo de gas auxiliar pueden oscilar entre 10-60 $\mathrm{mL} \mathrm{min}^{-1}$. El gas utilizado es nitrógeno (pureza de 99.999\%; Air Liquide). 


\subsection{Sistema de introducción de muestras-PTV-GC-MS}

\subsubsection{Sistema de introducción de muestras}

El sistema de introducción de muestras acoplado a la configuración PTV-GC-MS es un automuestreador MPS2 Multi Purpose Sampler de Gerstel (Mühlheim an der Ruhr, Alemania). Este automuestreador aporta una gran versatilidad al equipo, ya que permite seleccionar distintas modalidades de introducción de muestra (inyección de muestras líquidas, espacio de cabeza estático, microextracción con sorbentes empaquetados y microextracción en fase sólida) siendo la microextracción con sorbentes empaquetados la utilizada para la segunda metodología en el capítulo I (I.2) y la generación de espacio de cabeza la utilizada en las dos metodologías propuestas en el capítulo II de esta memoria. El dispositivo consiste en un brazo muestreador automático al que se pueden acoplar diferentes módulos, en los que va montada la jeringa apropiada, en función del tipo de introducción de muestra que se quiera realizar. Para el caso de MEPS se utilizó una jeringa de $100 \mu \mathrm{L}$ con un cartucho $\mathrm{C}_{18}$, ya descrita y mostrada en el apartado de técnicas de pretratamiento de muestra de esta introducción. Para la generación de espacio de cabeza se utilizó una jeringa termostatada de $2.5 \mathrm{~mL}$.

Este automuestrador también posee diferentes tipos de bandejas para los viales de muestra adecuados para cada aplicación, empleándose la bandeja con espacio para 32 viales para MEPS y HS.

El horno generador de espacio de cabeza tiene capacidad para seis viales, cuya temperatura puede programarse entre $35^{\circ} \mathrm{C}$ y $200{ }^{\circ} \mathrm{C}$. Este horno permite la agitación orbital de las muestras en un intervalo de velocidades de 
entre 250 y 750 rpm.

\subsubsection{Inyector de temperatura programada}

El PTV utilizado en esta configuración es un 6890 de Agilent Technologies equipado con un liner de iguales dimensiones y mismo sistema de calentamiento y enfriamiento que el descrito en la configuración anterior. En este caso el cabezal del inyector tiene septum, por lo que no es necesaria la válvula de sellado.

Con esta configuración se han utilizado liners con diferentes materiales de relleno dependiendo de las metodologías propuestas para las diferentes aplicaciones. De este modo, para la determinación de clorofenoles en suelos (I.2) así como para la determinación de aminoácidos en muestras de orina (II.1) se ha utilizado lana de vidrio, mientras que en la determinación de posibles biomarcadores en muestras de orina (II.2) se ha utilizado Tenax-TA ${ }^{\circledR}$.

\subsubsection{Cromatógrafo de gases}

El cromatógrafo de gases utilizado es un Agilent 7890A equipado con una columna capilar DB-VRX (20 m x $0.18 \mathrm{~mm}$ x $1 \mu \mathrm{m}$, rango de trabajo $-10 \mathrm{a}$ $260^{\circ} \mathrm{C}$ ) de Agilent Technologies (J\&W Scientific Columns, USA). El horno del cromatógrafo permite programar hasta cinco rampas de temperatura con diferentes velocidades. Las rampas máximas permitidas por el equipo son 70 ${ }^{\circ} \mathrm{C} \min ^{-1}$ hasta $175{ }^{\circ} \mathrm{C}, 45^{\circ} \mathrm{C} \min ^{-1}$ de 175 a $300{ }^{\circ} \mathrm{C}$ y $35^{\circ} \mathrm{C}$ min $^{-1}$ de 300 a 450 ${ }^{\circ} \mathrm{C}$. El gas portador utilizado es helio N50 (pureza de 99.999\%; Air Liquide).

\subsubsection{Espectrómetro de masas}

El espectrómetro de masas cuadrupolar es un Agilent 5975C inert XL 
MSD de Agilent Technologies (Waldbronn, Alemania). La fuente de ionización es de impacto electrónico, con un voltaje de ionización de 70 eV. Las temperaturas recomendadas para la fuente de ionización y el cuadrupolo son $230^{\circ} \mathrm{C}$ y $150^{\circ} \mathrm{C}$, respectivamente.

Es posible seleccionar tres modos de adquisición de datos: scan, en el que el detector hace un barrido de un amplio intervalo de masas, previamente especificado; el modo de seguimiento de iones seleccionados (selected ion monitoring, SIM), que permite seleccionar los iones característicos de las especies de interés, de modo que para cada intervalo de tiempo sólo se registran unos pocos iones seleccionados; y el modo SIM/scan sincrónico que realiza simultáneamente ambas modalidades.

La base de datos utilizada para la identificación de los compuestos es la NIST'98 (NIST/EPA/NIH Mass spectral Library, version 2.0). 


\section{CAPÍTULO I.}

DESARROLLO DE METODOLOGÍAS ANALÍTICAS PARA LA DETERMINACIÓN DE COMPUESTOS DE INTERÉS MEDIOAMBIENTAL EN MUESTRAS DE SUELO 

Líquidos iónicos como fases estacionarias en cromatografía de gases. Determinación de clorobencenos en suelos 

Artículo de investigación
Resumen 

El término "líquidos iónicos" hace referencia a un tipo de sales orgánicas que tienen un bajo punto de fusión. Su estructura química consiste en cationes orgánicos unidos por una cadena de carbono de longitud variable a los cuales se encuentran asociados aniones que pueden ser inorgánicos u orgánicos. Los líquidos iónicos se han usado en diferentes campos de la química analítica, incluyendo las separaciones analíticas. Las características especiales que poseen los hacen adecuados para su uso como fases estacionarias en columnas capilares para cromatografía de gases (GC). De estas especiales propiedades se puede destacar que no son volátiles y tienen alta estabilidad frente a la temperatura, por lo que las columnas que los contienen tienen, en principio, menor sangrado y una vida útil más larga; además, ya que son líquidos en un amplio intervalo de temperatura, se puede aumentar sustancialmente la temperatura de trabajo. Además de por todas estas características, el interés en su aplicación como fases estacionarias surge por sus propiedades cromatográficas específicas y mecanismos de retención derivados de su estructura molecular. Las fases estacionarias de líquidos iónicos parecen tener una doble naturaleza, esto es, son capaces de separar moléculas polares como si fueran fases estacionarias polares y moléculas apolares como si fueran fases estacionarias apolares. Estas fases estacionarias han sido empleadas en el análisis de una gran variedad compuestos, sin embargo, hasta donde sabemos, no habían sido aplicadas para análisis y determinación de clorobencenos.

Los clorobencenos forman un grupo de compuestos aromáticos cíclicos en los que uno o más átomos de hidrógeno del anillo de benceno han sido sustituidos por átomos de cloro. Estos compuestos se liberan al ambiente 
durante su fabricación o su utilización como intermedios en la producción de otros compuestos, o durante la eliminación de residuos en incineradoras y lugares de desechos peligrosos. Los clorobencenos usados como desodorantes, desengrasantes, insecticidas, herbicidas y defoliantes también se liberan al medio ambiente como resultado directo de su aplicación. Otra fuente directa de estos compuestos en los suelos es el uso de lodos de depuradora como abono en terrenos agrícolas; estos lodos contienen diversos compuestos químicos, entre los que pueden estar los clorobencenos, que pueden penetrar en el suelo.

Por todo ello, los objetivos de este trabajo fueron, por un lado, la evaluación de diferentes columnas de líquidos iónicos comercialmente disponibles con el fin de obtener la mejor resolución de los picos para los clorobencenos, así como la mayor simetría y, comparar los resultados con los de una columna convencional; por otro lado, el desarrollo de una metodología analítica basada en el uso de una de estas columnas de líquidos iónicos en GC con un micro-detector de captura electrónica para la determinación de clorobencenos en muestras de suelos. Para la extracción de estos compuestos del suelo se propone la utilización de una versión simplificada de QuEChERS en la que, debido a las características no grasas de las matrices de suelos, se elimina la etapa de limpieza mediante extracción en fase sólida dispersiva (d-SPE). La optimización de las variables relacionadas con esta versión de QuEChERS y el diseño de un método para dopar las muestras de suelos, supusieron objetivos adicionales necesarios para el desarrollo de un método completo. 
Con el fin de obtener muestras representativas de suelos dopados con los analitos, se probaron diferentes procedimientos. En el primero de ellos, se depositaron $20 \mathrm{~g}$ de suelo en un frasco ámbar, se añadió sobre él $1 \mathrm{~mL}$ de la disolución de clorobencenos preparada en acetato de etilo, se agitó la mezcla para conseguir una mejor homogenización, se dejó evaporar el disolvente a temperatura ambiente y después, se almacenó bajo refrigeración a $4{ }^{\circ} \mathrm{C}$ hasta su análisis. Los resultados revelaron una pérdida importante de los analitos más volátiles durante la evaporación del disolvente, impidiendo su adecuada absorción en el suelo. Los rendimientos se encontraron entre 2.0 y $8.0 \%$ para los analitos más volátiles y entre 17 y $40 \%$ para los menos volátiles.

Para mejorar el procedimiento, se realizó un nuevo experimento en el que el frasco se mantenía cerrado y en frío durante cinco días después de haber dopado el suelo. Después de este tiempo se abría para permitir la evaporación del disolvente y, finalmente, se almacenaba en frío hasta su análisis. En este caso, cuando se permitía interaccionar a los analitos con la matriz de suelo durante un tiempo apropiado, los valores de recuperación mejoraban considerablemente, con rendimientos que iban del 47 al 114\%.

Con el fin de seguir optimizando este último procedimiento se probaron diferentes periodos de tiempo (5, 7, 12 y 15 días) desde que el suelo era dopado hasta que era extraído y analizado. Para la optimización del proceso de extracción de QuEChERS se probaron varias proporciones de sales $\mathrm{NaCl} / \mathrm{MgSO}_{4}$ para cada uno de los periodos de tiempo; de esta forma se optimizaban a la vez el periodo de tiempo y el proceso de extracción de QuEChERS. Los resultados no mostraron diferencias significativas, por lo que finalmente se seleccionó un procedimiento en el que las muestras de suelos 
dopadas se almacenaban durante cinco días a $4{ }^{\circ} \mathrm{C}$, después se eliminaba el disolvente, y la extracción y análisis se realizaban siete días después de haber sido dopado. Para permitir una adecuada separación de fases en la extracción mediante QuEChERS se seleccionó la cantidad de $1 \mathrm{~g}$ de $\mathrm{MgSO}_{4}$. Con este procedimiento final optimizado los rendimientos (entre 75 y 106\%) eran reproducibles y apropiados para la determinación de estos compuestos en suelos.

El proceso de extracción QuEChERS tiene la limitación de no conseguir factores de preconcentración altos, por lo que para superar este inconveniente $\mathrm{y}$, teniendo en cuenta las características de volatilidad de los clorobencenos, el proceso de inyección de los extractos en el sistema cromatográfico se llevó a cabo mediante un inyector de temperatura programada (PTV) en el modo solvent vent. Se optimizaron las variables que afectan a este modo de inyección, como el tipo de relleno del liner, volumen de inyección y temperatura inicial a la que ocurre el venteo para la eliminación del disolvente (temperatura de venteo). Finalmente, los mejores resultados se obtuvieron con un liner con relleno de Tenax-TA ${ }^{\circledR}$, un volumen de $5 \mu \mathrm{L}$ y una temperatura de venteo de $15^{\circ} \mathrm{C}$.

En cuanto a la separación de los clorobencenos con las columnas de líquidos iónicos, se evaluaron cuatro columnas capilares con diferentes fases estacionarias de líquidos iónicos, dentro del proyecto SILEP de Supelco. De acuerdo al procedimiento descrito por Mondello y colaboradores (basado en los valores de la Constante de McReynolds) la fase estacionaria SLB-IL100 puede definirse como extremadamente polar, la SLB-IL82 como altamente polar, mientras que las columnas SLB-IL61 y SLB-IL59 se corresponden con 
columnas de polaridad media. Todas ellas proporcionaban el mismo perfil de elución de los clorobencenos, observándose un aumento de los tiempos de retención al disminuir la polaridad de la fase estacionaria. Además, se encontraron algunas diferencias entre ellas. La columna SLB-IL100 no permitía la correcta separación de los clorobencenos ya que, junto con la SLB-IL61 eran las que proporcionaban los valores menos adecuados de factor de asimetría. La mejor separación de algunos de los compuestos que se obtenía con la SLB-IL82, sumado al importante proceso de degradación después de varias inyecciones que se observó con las columnas SLB-IL59 y SLB-IL61 y que daba lugar a una pérdida de resolución, hicieron que la columna SLB-IL82 fuera la seleccionada para llevar a cabo la comparación con la columna convencional HP5.

El perfil de elución que se obtenía con la columna HP5 era diferente al de las fases estacionarias de líquidos iónicos, y estaba, en este caso, relacionado directamente con el punto de ebullición de los clorobencenos. Con la columna SLB-IL82 se obtenía una mejor separación de los compuestos que con la columna convencional, lo que hizo que se seleccionara la columna SLB-IL82 para proponer un método analítico para la determinación de los clorobencenos en muestras de suelos.

La existencia del efecto matriz es muy común en muchas de las técnicas analíticas utilizadas para la determinación de compuestos químicos en suelos, lo que hace necesario su estudio cuando se proponen metodologías nuevas de análisis. Para su evaluación se doparon a siete niveles de concentración cuatro suelos diferentes, se extrajeron y analizaron con el procedimiento propuesto y se compararon las pendientes de las rectas de 
calibración mediante un contraste de significación (test $t$-Student) para determinar si la diferencia entre las pendientes era significativa o no. Para la mayoría de los compuestos se encontraron diferencias significativas en las pendientes para los cuatro suelos, lo que demostraba la existencia de efecto de matriz. Además, la influencia del efecto de matriz era diferente para cada compuesto analizado. Estas diferencias pueden ser explicadas en términos de las diferentes interacciones de los compuestos en los cuatro tipos de suelos, que tienen una estructura de poro compleja y contienen diferentes proporciones de minerales y componentes orgánicos naturales. Por ello, para poder cuantificar estos analitos en muestras de suelos, se propuso realizar la calibración y predicción mediante el método de adición estándar.

Las características analíticas del método se determinaron con una muestra de suelo dopado. Debido a la complejidad de la matriz de los suelos, en algunos casos y dependiendo del tipo de suelo que se trate, puede ocurrir que la interferencia de los compuestos de la matriz con los analitos de interés impida la correcta cuantificación de estos últimos. Esto es lo que ocurre para los compuestos 1,2,3,4-TeCB y PeCB en el análisis de este suelo. Para el resto de los compuestos los calibrados mostraban comportamiento lineal con buenos valores de coeficiente de determinación. La validez de los modelos generados fue comprobada usando análisis de varianza y ninguno de ellos presentaba fallo de ajuste. La repetibilidad instrumental y la reproducibilidad fueron evaluadas a dos niveles de concentración, obteniéndose resultados altamente satisfactorios. Los límites de detección (LOD) y cuantificación (LOQ) se encontraban entre 0.1 y $4.7 \mu \mathrm{gg}^{-1}$ y entre 0.4 y $16 \mu \mathrm{gg}^{-1}$, respectivamente. Estos valores son inferiores a los obtenidos con técnicas de 
extracción clásicas y del mismo orden a los obtenidos con microextracción en fase sólida.

Para validar el método optimizado, se doparon dos suelos con los clorobencenos y se analizaron con el método de adición estándar. En el caso de estos dos suelos, a diferencia del suelo utilizado para evaluar las características analíticas del método, los compuestos 1,2,3,4-TeCB y PeCB se separaban y cuantificaban adecuadamente, lo que pone de manifiesto que la complejidad y diversidad de las matrices de suelos puede interferir en la cuantificación de los analitos de interés. En la mayoría de los casos, las concentraciones calculadas eran significativamente iguales a las concentraciones reales, de forma que, el intervalo de confianza de la predicción incluía la concentración real a la que se había dopado el suelo.

Como conclusiones podemos destacar que, de entre las columnas de líquidos iónicos evaluadas en este trabajo, la columna SLB-IL82 es la que proporciona los mejores valores de resolución y factor de asimetría para la separación de los clorobencenos y que, además, se ha optimizado y propuesto un método basado en su utilización en GC- $\mu$ ECD para la determinación de clorobencenos en suelos mediante su extracción con una versión simplificada de QuEChERS. Por otro lado, también se ha propuesto un procedimiento para dopar las muestras de suelo y se ha optimizado la extracción con QuEChERS. Los resultados alcanzados en este trabajo revelan la validez de la metodología propuesta para la determinación de clorobencenos en matrices de suelos. 

Artículo de investigación I.1

Journal of Separation Science 37 (2014) 1448-1455 



\section{Rosa María González Paredes \\ Carmelo García Pinto José Luis Perez Pavón Bernardo Moreno Cordero \\ Departamento de Química \\ Research Article \\ lonic liquids as stationary phases in gas chromatography: Determination of chlorobenzenes in soils} Analítica, Nutrición y

Bromatologia, Facultad de Ciencias Quimicas, Universidad de Salamanca, Salamanca, Spain

Received January 30, 2014 Revised March 26, 2014 Accepted March 27, 2014
The present research focuses on the evaluation of different ionic liquid (IL) stationary phases in gas chromatography. The different IL columns were evaluated in terms of peak resolution $\left(R_{\mathrm{s}}\right)$ and peak symmetry for the separation of the chlorobenzenes. The determination of chlorobenzenes in soil samples by means of the optimal IL stationary phase (SLB-IL82) is proposed as an application. Soil pretreatment was based on a simplified quick, easy, cheap, effective, rugged, and safe extraction procedure and a large injection volume via a programed temperature vaporizer working in solvent vent mode. The retention time of the chlorobenzenes increased as the polarity of the IL column decreased. SLB-IL82 is the stationary phase that provides the best values as regards $R_{\mathrm{s}}$ and asymmetry factor. Soil sample blanks were spiked with the analytes before subjecting the sample to the extraction process. The existence of a matrix effect was checked and the analytical characteristics of the method were determined in a fortified garden soil sample. The method provided good linearity, good repeatability and reproducibility values, and the LODs were in the $0.1-4.7 \mu \mathrm{g} / \mathrm{kg}$ range. Two fortified soil samples were applied to validate the proposed methodology.

Keywords: Chlorobenzenes / Gas chromatography / lonic liquid stationary phase / Quick, easy, cheap, effective, rugged and safe / Soil matrices DOI $10.1002 /$ jssc. 201400077

Additional supporting information may be found in the online version of this article at the publisher's web-site

\section{Introduction}

Ionic liquids (ILs) can be defined as organic salts with low melting points. They have a structure in which the organic cations are linked by a carbon chain of varying length and the corresponding anions are associated with them. ILs are used in different fields of analytical chemistry, including analytical separations, and can be used in many different applications [1-3]. The special characteristics of these ILs [4,5] make them suitable for the requirements of the capillary columns used in GC, making them ideal for use as stationary phases [6-9]. They are liquid over a broad temperature range such that the working temperature can be increased substantially. Additionally, they are not volatile and they have

Correspondence: Dr. Carmelo García Pinto, Departamento de Química Analítica, Nutrición y Bromatología, Facultad de Ciencias Químicas, Universidad de Salamanca, 37008 Salamanca, Spain E-mail:cgp@usal.es Fax: +34-923-294483

Abbreviations: AF, asymmetry factor; DCB, dichlorobenzene; $\mu \mathrm{ECD}$, microelectron capture detector; $\mathrm{HCB}$, hexachlorobenzene; IL, ionic liquid; PeCB, pentachlorobenzene; PTV, programed temperature vaporizer; QuEChERS, quick, easy, cheap, effective, rugged, and safe; $\boldsymbol{R}_{\mathbf{s}}$, peak resolution; TCB, trichlorobenzene; TeCB, tetrachlorobenzene high thermal stability, and hence columns containing them are subject to less bleeding and have a longer lifetime.

Interest in their application as stationary phases arises from their specific chromatographic properties [10] and retention mechanisms mainly due to their molecular struc ture $[11,12]$. It has been stated that IL stationary phases seem to have a dual nature, i.e. they are capable of separating polar molecules as if they are polar stationary phases and nonpola molecules as if they are nonpolar stationary phases [13]. An excellent review article about IL stationary phases for GC has been recently published [14].

IL stationary phases have been employed in the analysis of essential oils $[15,16]$, fatty acids [17-19], benzothiazoles and benzotriazoles [20], nitrosamines and caffeine metabolites [21], and haloanisoles [22]. Different IL columns have been employed in multidimensional GC applications, such as in the determination of fatty acids [19], polychlorinated biphenyls [23,24], allergens [25], or sulfur-containing compounds [26].

Chlorobenzenes form a group of cyclic aromatic compounds in which one or more of the hydrogen atoms of the benzene ring have been replaced by a chlorine atom The natural sources of chlorobenzenes in the environment

Colour Online: See the article online to view Fig. 1 in colour. 
have not been identified but these compounds are released to the environment during their manufacture or their use as intermediates in the production of other chemicals. They are also released during the disposal of chlorobenzene products, such as from incinerators and hazardous waste sites. Chlorobenzenes used as deodorizers, fumigants, degreasers, insecticides, herbicides, and defoliants are also released to the environment as a direct result of their application $[27,28]$. Another direct source of these compounds in soils is the use of sewage sludge as fertilizer on agricultural land; these sewage sludges contain diverse chemical compounds, which may also enter the soil matrix $[29,30]$.

In the present work, different commercially available IL columns were evaluated and compared with a conventional stationary phase in order to obtain the best peak resolution $\left(R_{\mathrm{s}}\right)$ for the target analytes as well as the highest peak symmetry. We also developed an analytical methodology based on the use of the IL stationary phase SLB-IL82 in GC with microelectron capture detector $(\mathrm{GC}-\mu \mathrm{ECD})$ for the determination of chlorobenzenes in soil samples by means of simplified quick, easy, cheap, effective, rugged, and safe (QuEChERS) extraction. This simplified version of the QuEChERS method [31] eliminates the dispersive SPE cleanup step after extraction due to the nonfatty characteristics of soil matrices and hence involves fewer treatment stages of the sample, which makes the final procedure simpler, faster, and cheaper and minimizes the errors associated with this step. A procedure to spike soil samples was designed to obtain representative soil samples, and the variables related to the simplified QuEChERS extraction were selected according to the results published in a previous paper [31]. The existence of a matrix effect was checked with four types of soils and the analytical characteristics of the method were determined in a spiked garden soil sample (soil 1). Two soils (soils 2 and 4 ) were applied to validate the proposed methodology.

\section{Materials and methods}

\subsection{Chemicals}

Ethyl acetate was of HPLC grade (CHROMASOLV ${ }^{\circledR}$ Plus) and was purchased from Sigma-Aldrich (Steinheim, Germany). Analytical standards of chlorobenzenes (1,2dichlorobenzene (1,2-DCB) 1,3-dichlorobenzene (1,3-DCB), 1,4-dichlorobenzene (1,4-DCB), 1,2,3-trichlorobenzene (1,2,3-TCB), 1,2,4-trichlorobenzene (1,2,4-TCB), 1,3,5trichlorobenzene (1,3,5-TCB), 1,2,3,4-tetrachlorobenzene (1,2,3,4-TeCB), 1,2,3,5-tetrachlorobenzene (1,2,3,5-TeCB), 1,2,4,5-tetrachlorobenzene (1,2,4,5-TeCB), pentachlorobenzene, and hexachlorobenzene) ( $99 \%$ purity) were from Sigma-Aldrich: Anhydrous magnesium sulfate (extra pure) and sodium chloride (reagent grade) were from Scharlau (Barcelona, Spain). Ultrapure water used was obtained with an Elgastat UHQ water purification system.

\subsection{Standard solutions and samples}

Stock solutions (1000 mg/L in ethyl acetate) of each compound were prepared and stored in a refrigerator at $4^{\circ} \mathrm{C}$. From these, different solutions were prepared by dilution. They were used to optimize the experimental conditions, as well as in the spiking of soils at the required concentration levels.

Soil matrices were used for the optimization of the sample pretreatment step to check the existence of a matrix effect and determine the analytical characteristics of the method. Four different types of soils were used: a sandy loam soil (soil 1) from a public garden (Salamanca, Spain) and three different soils from Badajoz, Spain: a loam soil (soil 2), a sandy soil (soil 3), and clay loam (soil 4); soils 2 and 4 were taken from agricultural cropland. Chemical properties of these soils are shown in Supporting Information Table S1.

The procedure used to spike the samples was as follows: $20 \mathrm{~g}$ of soil was placed in a $100 \mathrm{~mL}$ flask and $1 \mathrm{~mL}$ of a chlorobenzene solution in ethyl acetate was added (at a suitable concentration for each case). The flask was sealed hermetically and shaken vigorously for $15 \mathrm{~min}$ to achieve perfect homogenization of the compounds in the matrix. The samples were stored in a refrigerator $\left(4^{\circ} \mathrm{C}\right)$ for five days to allow the interaction between the compounds and the matrix to take place. After five days, the flask was opened to allow the dissolvent to evaporate off $(6 \mathrm{~h})$. Then, the flask was closed and stored at $4^{\circ} \mathrm{C}$ again for a further two days.

\subsection{Apparatus}

Injection of the samples was carried out with an automatic liquid sample injection system (Agilent Technologies 7683: Waldbronn, Germany) with a $10 \mu \mathrm{L}$ syringe. The sample was transferred to the GC system by means of an Agilent Technologies 6890 programmable temperature vaporizer (PTV) inlet (CIS-4). Cooling was accomplished with $\mathrm{CO}_{2}$. The PTV was equipped with a $71 \mathrm{~mm} \times 2 \mathrm{~mm}$ liner (internal volume of $180 \mu \mathrm{L})$ packed with Tenax-TA ${ }^{\circledR}$, a hydrophobic polymer designed to trap organics. The carrier gas was helium $(99.999 \%$ pure, Air Liquide). GC analysis was performed using an Agilent Technologies 7890A GC system equipped with a ${ }^{63} \mathrm{Ni}$ microelectron capture detector $(\mu \mathrm{ECD})$.

\subsection{Evaluated columns}

Four commercially available IL columns of $30 \mathrm{~m} \times 0.25 \mathrm{~mm}$ id coated with a $0.20 \mu \mathrm{m}$ film thickness of different stationary phases (i.e. SLB-IL59, SLB-IL61, SLB-IL82, and SLB-IL100) were obtained from Supelco. The chemical composition of these IL columns is shown in Supporting Information Table S2. According to the supplementary information provided by the supplier, these stationary phases have different compositions and polarity as given by the polarity index derived from McReynolds constants (normalized to SLB-IL100). To compare the results with a conventional 
stationary phase, an HP5 column $(30 \mathrm{~m} \times 0.32 \mathrm{~mm}$ id $\times$ $0.25 \mu \mathrm{m}$ ) from Agilent Technologies was used.

The criteria parameters in the chromatographic separation with ILs columns were to obtain the highest $R_{\mathrm{s}}$ between the different analytes, suitable peak symmetry, and the shortest analysis time. The asymmetry factor $(\mathrm{AF})$ and $R_{\mathrm{s}}$ were calculated according to previously reported equations $[32,33]$.

\subsection{Analytical procedure}

For the simplified QuEChERS approach, $2.5 \mathrm{~g}$ of soil sample was weighed in a $15 \mathrm{~mL}$ glass centrifuge tube with a screw cap, which keeps the tube closed for most of the process of sample preparation, thus avoiding losses of volatile compounds during this stage as much as possible. A total of $1.5 \mathrm{~mL}$ of ultrapure water was added to the soil sample in order to render the pores in the sample more accessible to the extraction solvent and to homogenize the water content in different soil samples, and the mixture was shaken for 1 min with a vortex device. Then, $2.5 \mathrm{~mL}$ of ethyl acetate (extraction solvent) was added and the mixture was shaken again for $1.5 \mathrm{~min}$. Following this, $1 \mathrm{~g}$ of magnesium sulfate (prepared in advanced in a closed vial) was added, shaking the mixture for $1 \mathrm{~min}$ as fast as possible to prevent the formation of $\mathrm{MgSO}_{4}$ conglomerates. The tube was centrifuged at $5000 \mathrm{rpm}$ for $5 \mathrm{~min}$. Finally, the organic layer was filtered with a $0.45 \mu \mathrm{m}$ nylon filter and transferred to an autosampler vial for $\mathrm{GC}$ analysis and subsequent measurement. A volume of $5 \mu \mathrm{L}$ of the organic extract (in solvent vent mode) was injected. During injection, accomplished at a rate of $5 \mu \mathrm{L} / \mathrm{s}$, the split valve was open, PTV was set at $15^{\circ} \mathrm{C}$, and vent flow was adjusted to $50 \mathrm{~mL} / \mathrm{min}$. The purge time was set at $0.5 \mathrm{~min}$, after which the splitless mode was programed for $1.5 \mathrm{~min}$ while the temperature was increased at a rate of $12^{\circ} \mathrm{C} / \mathrm{s}$ to $250^{\circ} \mathrm{C}$, such that the analytes were transferred to the chromatographic system. The injection time was fixed at $1.5 \mathrm{~min}$.

The conditions for the chromatographic measurements were optimized and the SLB-IL82 IL stationary phase was selected. The carrier gas used was helium at a rate of $1.5 \mathrm{~mL} / \mathrm{min}$. The oven temperature program started at $40^{\circ} \mathrm{C}$ for $1 \mathrm{~min}$, increased at a rate of $20^{\circ} \mathrm{C} / \mathrm{min}$ to $140^{\circ} \mathrm{C}$, then $3^{\circ} \mathrm{C} /$ min to $150^{\circ} \mathrm{C}$, and finally $100^{\circ} \mathrm{C} / \mathrm{min}$ to $170^{\circ} \mathrm{C}$, which was held for $5 \mathrm{~min}$.

The parameters of the $\mu$ ECD detector were as follows: a detection temperature of $260^{\circ} \mathrm{C}$ and a make-up flow gas $\left(\mathrm{N}_{2}\right)$ of $10 \mathrm{~mL} / \mathrm{min}$. Data acquisition was performed with Chemstation G2075BA version B.03.01 software from Agilent Technologies.

\section{Results and discussion}

\subsection{Preparation of spiked soil samples and} optimization of the extraction process

To obtain a representative soil sample spiked with the analytes of interest, different procedures were assayed. On the first one, $20 \mathrm{~g}$ of clean soil was weighed and placed in an amber glass vial, then adding an aliquot $(1 \mathrm{~mL})$ of a solution of chlorobenzenes in ethyl acetate and shaking the mixture vigorously to achieve sample homogenization; the solvent was allowed to evaporate off naturally and then stored under refrigeration at $4^{\circ} \mathrm{C}$ until analysis. The results revealed an important loss of the more volatile analytes during the evaporation of the solvent, hindering proper absorption of these by the soil matrix. The extraction yields were between 2.0 and $8.0 \%$ for most of the more volatile analytes and between 17 and $40 \%$ for the less volatile ones.

To overcome this drawback, a new experiment was performed in which the vial is closed for five days after spiking the soil; later the vial is open to allow the evaporation of the solvent and, finally, it is stored under refrigeration until analysis. In this case, when the analytes were allowed to interact over an appropriate time with the soil matrix, the recovery values improved considerably with extraction yields ranged between 47 and $114 \%$.

Different times $(5,7,12$, and 15 days) and different $\mathrm{NaCl} / \mathrm{MgSO}_{4}$ ratios were studied for the optimization of the QuEChERS extraction process and the results did not show significant differences. So, a QuEChERS procedure was chosen in which a spiked soil is stored over five days at $4^{\circ} \mathrm{C}$; then, the solvent was removed and extraction was performed 7 days after spiking. In the extraction process, $1 \mathrm{~g}$ of $\mathrm{MgSO}_{4}$ was added to achieve suitable phase separation. Supporting Information Fig. S1 shows the results of these experiments. These extraction yields (between 75 and 106\%) are reproducible and suitable for the determination of these compounds in soils.

\subsection{Experimental chromatographic and detection} variables

The QuEChERS extraction process is limited by the fact that high preconcentration factors cannot be achieved. To circumvent this drawback, and bearing in mind the volatility characteristics of chlorobenzenes, extract injection into the chromatographic system was carried out using a programed temperature vaporizer (PTV). A liner packed with Tenax-TA ${ }^{\circledR}$ and an empty liner were evaluated. The highest signals were obtained with Tenax-TA ${ }^{\circledR}$ (Supporting Information Fig. S2). Solvent vent injection mode allows the injection of large sample volumes. For this, injection volumes of 5, 10, 15, 20, and $25 \mu \mathrm{L}$ were studied. As the volume was increased, the signal from the analytes increased; however, the chromatographic peaks also widened. As a result, the separation of 1,2,3,5-TeCB, 1,2,3-TCB, and 1,2,4,5-TeCB (peaks 6, 7, and 8 , respectively) was affected. Figure 1 shows the partial chromatograms of the most affected compounds with the different injection volumes. In light of these, an injection volume of $5 \mu \mathrm{L}$ was selected.

The initial temperature of the liner in which solven purging occurs, or venting temperature, was studied for values of $10,15,20$, and $50^{\circ} \mathrm{C}$. The areas of the signals decreased gradually with increasing initial temperature 


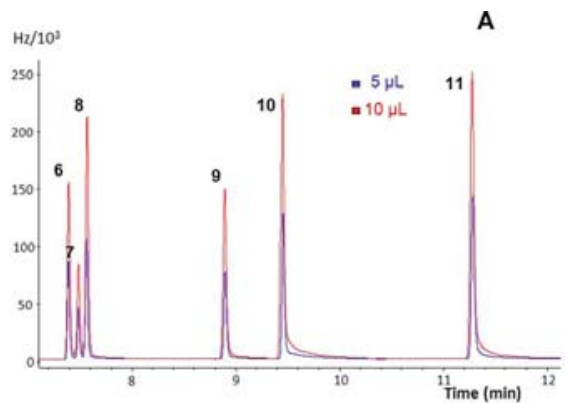

Table 1. Relative retention $(r)$ and peak resolution $\left(R_{\mathrm{s}}\right)$ of the chlorobenzenes analyzed

\begin{tabular}{|c|c|c|c|c|c|c|c|c|}
\hline \multirow[t]{2}{*}{ Analyte } & \multicolumn{2}{|c|}{ SLB-IL59 } & \multicolumn{2}{|c|}{ SLB-IL61 } & \multicolumn{2}{|c|}{ SLB-IL82 } & \multicolumn{2}{|c|}{ SLB-IL100 } \\
\hline & $r$ & $R_{\mathrm{s}}$ & $r$ & $R_{\mathrm{s}}$ & $r$ & $R_{\mathrm{s}}$ & $r$ & $R_{\mathrm{s}}$ \\
\hline DCE & 1.00 & & 1.00 & & 1.00 & & 1.00 & \\
\hline --DCB & 1.05 & 5.63 & 1.04 & .03 & 1.04 & 36 & .05 & 3. \\
\hline ,5-ТСВ & & & 1.05 & 0.84 & & & & \\
\hline 2-DC & & 7.34 & 1. & .84 & 1.12 & 9.24 & 14 & 5.5 \\
\hline $2,4-\mathrm{TT}$ & 1.24 & 16.59 & 27 & 10.79 & 1.22 & 17.60 & 28 & 9.0 \\
\hline $1,2,3,5-1$ & 1.35 & 15.99 & 1.43 & 10.76 & 1.34 & 19.17 & 1.47 & 12.82 \\
\hline 2,7 & & & & & & 2.44 & 1.48 & 0.9 \\
\hline , 2,3-T & 1. & 1.25 & 1.5 & 0 & 1.37 & 2.09 & & \\
\hline$, 3,4-\mathrm{TeC}$ & 1.56 & 23.99 & 1.95 & 21.83 & 1.62 & 30.45 & 1.66 & 12.65 \\
\hline CB & 1. & . & 2.08 & 5.11 & 1.71 & 12.93 & 1.72 & 5.8 \\
\hline D. & 1.98 & 33.45 & 2.49 & 17.96 & 2.04 & 44.44 & 1.94 & 18. \\
\hline
\end{tabular}

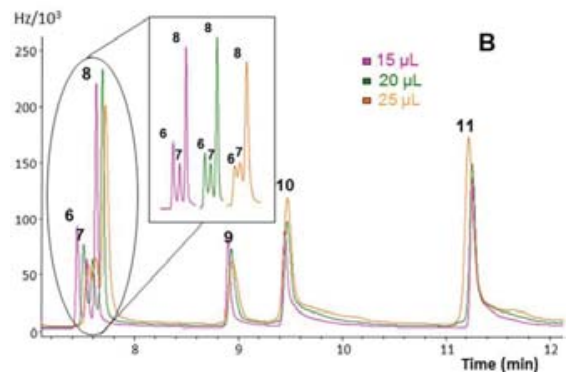

Figure 1. $G C-\mu E C D$ chromatograms of a standard solution of the target compounds: (A) with an injection volume of 5 and $10 \mu \mathrm{L}$, (B) with an injection volume of 15,20 , and $25 \mu \mathrm{L}$. (1) 1,3-DCB; (2) 1,4-DCB; (3) 1,3,5-TCB; (4) 1,2-DCB; (5) 1,2,4-TCB; (6) 1,2,3,5TeCB; (7) 1,2,4,5-TeCB; (8) 1,2,3-TCB; (9) 1,2,3,4-TCB; (10) PeCB; (11) $\mathrm{HCB}$.

(Supporting Information Fig. S3). However, with a temperature of $10^{\circ} \mathrm{C}$, problems in reproducibility were observed for the less-retained compounds, probably due to the difference between the initial temperature of the PTV and initial oven temperature. Accordingly, a value of $15^{\circ} \mathrm{C}$ was chosen.

Four different IL columns were evaluated for chlorobenzenes determination. According to the procedure proposed by Mondello et al. (based on the McReynolds constant values) [15], the SLB-IL100 phase can be defined as an extremely polar one, SLB-IL82 as a highly polar one, and SLB-IL61 and SLB-IL59 can be classified as being of medium or mediumhigh polarity. Temperature programing was selected depending on the separation of chlorobenzenes in each column (Supporting Information Table S3).

The different IL columns evaluated afforded the same elution profile, with retention times $<14$ min. The elution profile normalized to the 1,3-DCB retention time (first analyte eluted) and the $R_{\mathrm{s}}$ are shown in Table 1. Some variations between the different IL columns were observed. The analytes 1,4-DCB and 1,3,5-TCB coeluted in the SLB-IL59, SLB-IL82, and SLB-IL100 phases. Furthermore, 1,2,4,5-TeCB

and 1,2,3-TCB also coeluted in the SLB-IL100 column, and hence this column does not afford an appropriate separation of chlorobenzenes. Although in the SLB-IL61 column, 1,4 DCB and 1,3,5-TCB showed a slight degree of separation, the separation between these analytes was unsuitable, as seen from the $R_{\mathrm{S}}(0.84)$. The least suitable values for the AF (Supporting Information Table S4) were obtained with the SLBgIL61 and SLB-IL100 columns. On comparing SLB-IL59 and SLB-IL82, the AF values were similar and all the values were close to unity. Nevertheless, the best $R_{\mathrm{s}}$ values were obtained with the SLB-IL82 column.

Figure 2 shows the chromatograms obtained for the separation of the standard components with the SLB-IL59, SLBIL82, and SLB-IL100 columns. As expected, the retention time increased as the polarity of the column decreased. SLB-IL82 showed a better separation of 1,2,3,5-TeCB, 1,2,4,5-TeCB, and 1,2,3-TCB (peaks 6, 7, and 8, respectively) than SLB-IL59. Moreover, some of the stationary phases, mainly SLB-IL59 and SLB-IL61, undergo an important degradation process after a few injections leading to a significant loss of $R_{\mathrm{s}}$. Accordingly, the SLB-IL82 column was selected to compare IL stationary phases with the conventional HP5 stationary phase.

Figure 3 shows the chromatograms obtained with the SLB-IL82 and HP5 columns under the same chromatographic conditions. The HP5 column provided a different elution profile of the IL stationary phases, in this case directly related to the boiling point of chlorobenzenes. In the HP5 column, coelution of 1,2,3,5-TeCB and 1,2,4,5-TeCB was observed (peaks 6 and 7, respectively). Separation of the same group of compounds, reported in the literature, with conventional stationary phases shows that the separation of the $1,2,3,5-\mathrm{TeCB}$ and $1,2,4,5-\mathrm{TeCB}$ analytes does not reach the baseline $[34,35]$ or a complete coelution is produced $[36,37]$. Only those studies that do not include the 1,2,3,5-TeCB do not present this problem [38-40]. Moreover, in our case, the separation of the 1,3-DCB and 1,4-DCB analytes (peaks 1 and 2 , respectively) in the conventional stationary phase did not reach the baseline. 

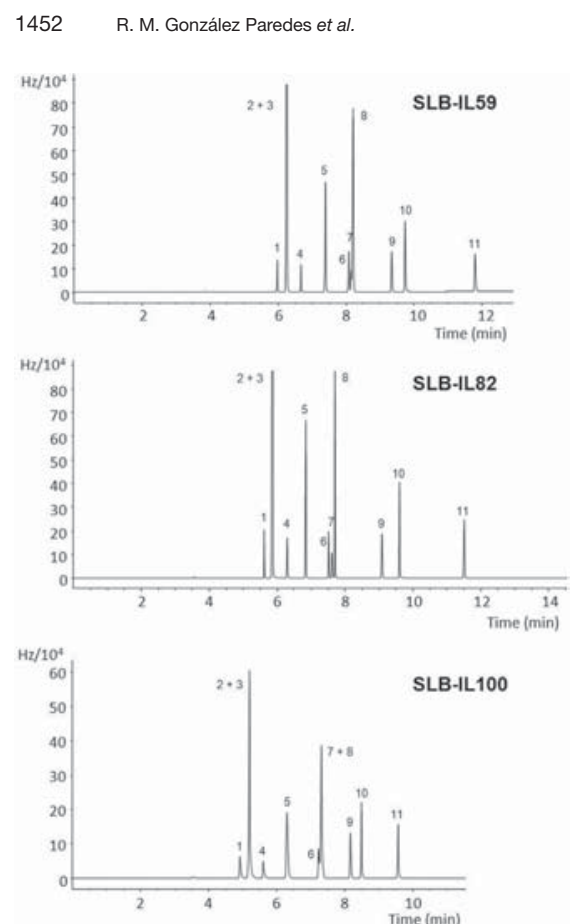

Figure 2. GC-PTV $-\mu E C D$ chromatograms of a standard solution of the target compounds from the SLB-IL59, SLB-IL82, and SLBIL100 columns. Peak assignment as in Fig. 1.

Both columns show a similar separation of chlorobenzenes; however, the differences discussed above, along with the aim of using IL stationary phases for the analysis of the environmental samples, led us to select the SLB-IL82 column to propose an analytical method for the determination of chlorobenzenes in soil samples.

For the $\mu \mathrm{ECD}$ detector, the temperature and make-up flow parameters were optimized. The S/Ns were calculated for temperature values of $250,260,270,280$, and $290^{\circ} \mathrm{C}$ at the detector. The $\mathrm{S} / \mathrm{Ns}$ of the compounds were higher for a temperature of $260^{\circ} \mathrm{C}$ and hence this was the value selected.

The make-up flow was studied for values of 10,20 , and $30 \mathrm{~mL} / \mathrm{min}$. It was observed that the $\mathrm{S} / \mathrm{N}$ decreased progressively with increasing make-up flow. Accordingly, a make-up flow of $10 \mathrm{~mL} / \mathrm{min}$ was chosen.

\subsection{Matrix effect}

The existence of a matrix effect is very common in many of the analytical techniques used for the determination of chem-
J. Sep. Sci. $2014,37,1448-1455$
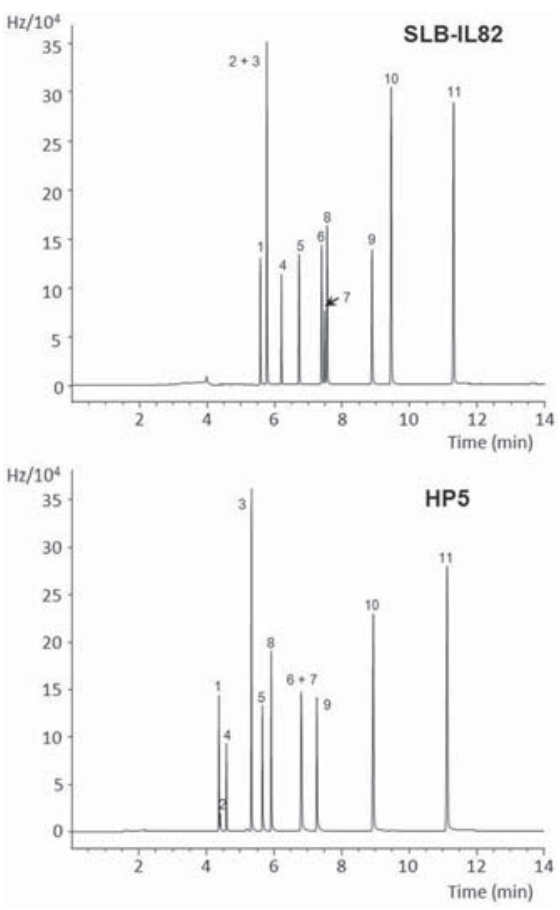

Figure 3. $G C-\mu E C D$ chromatograms of a standard solution of the target compounds from the SLB-IL82 and HP5 columns. Peak assignment as in Fig. 1.

icals in soils. To evaluate the possible existence of a matrix effect when the proposed procedure was applied to determine chlorobenzenes in soils, four different soil samples spiked at seven different concentration levels for each compound were extracted. The organic extracts obtained from the soil samples were analyzed with the optimized GC method (four injections for each level), and the slopes of the calibration curves were compared by Student's $t$-test to determine whether the slopes were significantly different from each other.

Only for some compounds and certain soil pairs, the calculated value of $t$ was less than the critical value and there was no matrix effect (Supporting Information Table S5). Thus, for most of the compounds, there were significant differences in slope for the four matrices considered and the matrix effect was present. Moreover, the influence of the matrix was different for each of the compounds. These differences could be explained in terms of the different interactions of the compounds in the four types of soils, which have a complex porous structure and contain different proportions of minerals and natural organic components. To overcome this 
Table 2. Analytical characteristic of the method in garden soil (soil 1)

\begin{tabular}{|c|c|c|c|c|c|c|c|c|c|c|}
\hline & \multirow[t]{2}{*}{ Slope } & \multirow[t]{2}{*}{$R^{2}$} & \multicolumn{2}{|c|}{$\begin{array}{l}\text { Concentration level } \\
(\mu \mathrm{g} / \mathrm{kg})\end{array}$} & \multicolumn{2}{|c|}{$\begin{array}{l}\text { Instrumental repeatability } \\
\text { (RSD\%) }\end{array}$} & \multicolumn{2}{|c|}{ Reproducibility (RSD\%) } & \multirow[t]{2}{*}{$\begin{array}{l}\mathrm{LOD} \\
(\mu \mathrm{g} / \mathrm{kg})\end{array}$} & \multirow[t]{2}{*}{$\begin{array}{l}\mathrm{LO0} \\
(\mu \mathrm{g} / \mathrm{kg})\end{array}$} \\
\hline & & & Low level & High level & Low level & High level & Low level & High level & & \\
\hline 1,3-DCB & $(4.9 \pm 0.2) 10$ & 0.9917 & 50 & 150 & 8.1 & 13.5 & 17.7 & 3.4 & 4.7 & 16 \\
\hline $\begin{array}{l}1,4-\mathrm{DCB}+ \\
1,3,5-\mathrm{TCB}\end{array}$ & $(4.3 \pm 0.2) 10^{2}$ & 0.9922 & $20+20$ & $60+60$ & $5.5^{*}$ & $5.8^{*}$ & $1.9^{*}$ & $2.0^{*}$ & $0.6^{*}$ & $1.9^{*}$ \\
\hline $1,2-\mathrm{DCB}$ & $(5.3 \pm 0.2) 10$ & 0.9938 & 50 & 150 & 10.8 & 4.9 & 3.4 & 2.6 & 3.0 & 10 \\
\hline 1,2,4-ТCB & $(3.9 \pm 0.1) 10^{2}$ & 0.9953 & 20 & 60 & 5.7 & 4.8 & 3.3 & 1.5 & 4.2 & 14 \\
\hline $1,2,3,5-\mathrm{TeCB}$ & $(10.3 \pm 0.2) 10^{2}$ & 0.9988 & 5 & 15 & 6.8 & 3.3 & 8.6 & 4.8 & 0.4 & 1.4 \\
\hline $1,2,4,5-\mathrm{TeCB}$ & $(5.0 \pm 0.3) 10^{2}$ & 0.9828 & 5 & 15 & 5.9 & 5.4 & 11.4 & 4.2 & 0.9 & 2.9 \\
\hline 1,2,3-TCB & $(6.7 \pm 0.3) 10^{2}$ & 0.9893 & 8 & 24 & 8.1 & 4.5 & 6.6 & 2.3 & 0.7 & 2.4 \\
\hline $1,2,3,4-\mathrm{TeCB}$ & - & - & 5 & 15 & - & - & - & - & - & - \\
\hline $\mathrm{PeCB}$ & - & - & 5 & 15 & - & - & - & - & - & - \\
\hline HCB & $(3.4 \pm 0.1) 10^{3}$ & 0.9941 & 5 & 15 & 7.0 & 3.4 & 17.7 & 4.2 & 0.1 & 0.4 \\
\hline
\end{tabular}

*, Calculated values for the sum of the two coeluting compounds.

-, Interference with the matrix compounds.

matrix effect, a standard additions protocol was proposed that has already been shown to work properly for soil matrices $[36,41,42]$.

\subsection{Analytical characteristics of the method}

In order to determine the analytical characteristics of the method, the garden soil sample (soil 1), spiked at seven concentration levels (ranged between 8 and $150 \mu \mathrm{g} / \mathrm{kg}$ ) was selected. The standard additions were performed exactly as the spiking procedure describe in Section 3.1. Each concentration level was subjected to the extraction procedure, and each of the extracts obtained was analyzed four times. All the calibrations showed linear behavior, with good values of the coefficient of determination $\left(R^{2}\right)$, except in the cases of $1,2,3,4$ $\mathrm{TeCB}$ and pentachlorobenzene discussed above (Table 2) for which the interference of the matrix compounds with the analytes of interest prevents their correct quantification. The validity of the models generated was checked using analysis of variance, and none of the models generated was found to show lack of fit.

Instrumental repeatability and reproducibility were determined at two concentration levels for the garden soil (soil 1). Instrumental repeatability was evaluated by injecting an extract ten times for each level. The reproducibility of the extraction procedure was calculated by injecting ten different extracts for each level. These extracts were injected three times. The values (expressed as RSD) are shown in Table 2 and were highly satisfactory.

The LODs of the proposed method ranged from 0.1 to $4.7 \mu \mathrm{g} / \mathrm{kg}$, whereas the LOQs ranged from 0.4 to $16 \mu \mathrm{g} / \mathrm{kg}$. These values are lower than those obtained with classical extraction techniques, such as Soxhlet extraction [43,44], and are of the same order as the values obtained with solid-phase microextraction [45].
Table 3. Determination of chlorobenzenes in soils 2 and 4 with SLB-IL82

\begin{tabular}{|c|c|c|c|c|}
\hline \multirow[t]{2}{*}{ Analyte } & \multicolumn{2}{|l|}{ Soil 2} & \multicolumn{2}{|l|}{ Soil 4} \\
\hline & $\begin{array}{l}\text { Actual } \\
\text { concentration } \\
(\mu \mathrm{g} / \mathrm{kg})\end{array}$ & $\begin{array}{l}\text { Calculated } \\
\text { value } \\
(\mu \mathrm{g} / \mathrm{kg})\end{array}$ & $\begin{array}{l}\text { Actual } \\
\text { concentration } \\
(\mu \mathrm{g} / \mathrm{kg})\end{array}$ & $\begin{array}{l}\text { Calculated } \\
\text { value } \\
(\mu \mathrm{g} / \mathrm{kg})\end{array}$ \\
\hline 1,3-DCB & 74 & $69 \pm 8$ & 74 & $76 \pm 5$ \\
\hline 1,4-DCB & 30 & $62 \pm 6$ & 30 & $51 \pm 5$ \\
\hline $1,3,5$-ТCB & 30 & & 30 & \\
\hline $1,2-\mathrm{DCB}$ & 74 & $73 \pm 9$ & 75 & $78 \pm 7$ \\
\hline $1,2,4-\mathrm{TCB}$ & 30 & $32 \pm 3$ & 30 & $29 \pm 2$ \\
\hline $1,2,3,5-\mathrm{TeCB}$ & 7.4 & $7.1 \pm 0.6$ & 7.4 & $7.6 \pm 0.8$ \\
\hline $1,2,4,5-\mathrm{TeCB}$ & 7.4 & $6.8 \pm 0.6$ & 7.4 & $7.3 \pm 0.7$ \\
\hline $1,2,3-\mathrm{TCB}$ & 12 & $11 \pm 1$ & 12 & $12 \pm 1$ \\
\hline $1,2,3,4-\mathrm{TeCB}$ & 7.4 & $7.6 \pm 0.9$ & 7.4 & $7.2 \pm 0.7$ \\
\hline PeCB & 7.4 & $6.1 \pm 0.8$ & 7.4 & $7.4 \pm 0.9$ \\
\hline HCB & 7.4 & $8.2 \pm 0.9$ & 7.5 & $9 \pm 1$ \\
\hline
\end{tabular}

\subsection{Method validation}

To validate the optimized method, two soils (soils 2 and 4 ) were spiked with all the chlorobenzenes and analyzed with the SLB-IL82 column.

The above-mentioned standard additions protocol was proposed for the accurate determination of chlorobenzenes. A six-level calibration study was performed, analyzing four replicates from each level. Table 3 shows the calculated concentration and the actual concentration for all the chlorobenzenes in the spiked soils (soils 2 and 4), which were analyzed with SLB-IL82. In these two soils, unlike soil 1, all the compounds were properly separated and quantified, which shows that the complexity and diversity of soil matrices may interfere in the quantification of the analytes of interest. In most 
cases, the calculated concentrations for soils 2 and 4 were close to the actual concentrations at which the samples were spiked, such that the confidence interval of prediction included the actual concentration of the soil. This reveals the validity of the methodology proposed for the determination of these compounds in soil matrices.

\section{Concluding remarks}

The evaluation of different IL stationary phases and their comparison with a conventional column has been carried out. It is important to note that, despite the high thermal stability of the ILs, some of the stationary phases undergo a clear degradation process with time leading to significant loss of $R_{\mathrm{s}}$. Among the IL columns evaluated in this work, SLBIL82 is the one that provides the best values as regards $R_{\mathrm{S}}$ and AF for the separation of these compounds. The method has been applied to determine chlorobenzenes in soil samples based on extraction with a simplified QuEChERS setup, and analysis by GC with the SLB-IL82 IL stationary phase has been proposed.

With the proposed procedure for spiking soil samples, representative soil samples and adequate recoveries are obtained. The analytical characteristics of the method were calculated for a spiked garden soil sample, with the observation of linear behavior, no lack of fit, suitable reproducibility and instrumental repeatability, and LODs $(0.1-4.7 \mu \mathrm{g} / \mathrm{kg})$ of the same order as the values obtained with other extraction procedures. The validation of the proposed method was carried out determining the concentration of chlorobenzenes in two spiked soils. The calibration strategy used was the standard additions protocol, and the results obtained were highly satisfactory.

Financial support from the DGI (CTQ2010-17514) is acknowledged. R. M. G. P. is grateful for her predoctoral grant from the University of Salamanca. The authors gratefully acknowledge Sigma-Aldrich for the supply of chromatographic columns through the SILEP project (Supelco Ionic Liquid Evaluation Project).

The authors have declared no conflict of interest.

\section{References}

[1] Liu, J., Jönsson, J. A., Jiang, G., TrAC Trends Anal. Chem. 2005, 24, 20-27.

[2] Sun, P., Armstrong, D. W., Anal. Chim. Acta 2010, 661 , $1-16$.

[3] Shamsi, S. A., Danielson, N. D., J. Sep. Sci. 2007, 30, 1729-1750.

[4] Anderson, J. L., Ding, R., Ellern, A., Armstrong, D. W., J. Am. Chem. Soc. 2005, 127, 593-604.

[5] Breitbach, Z. S., Armstrong, D. W., Anal. Bional. Chem. 2008, 390, 1605-1617
6] Berthod, A., Ruiz-Ángel, M. J., Carda-Broch, S., J. Chromatogr. A 2008, 1184, 6-18.

[7] Anderson, J. L., Armstrong, D. W., Anal. Chem. 2003, 75, 4851-4858.

[8] Payagala, T., Zhang, Y., Wanigasekara, E., Huang, K., Breitbach, Z. S., Sharma, P. S., Sidisky, L. M., Armstrong, D. W., Anal. Chem. 2009, 81, 160-173.

[9] Huang, K., Zhang, X., Armstrong, D. W., J. Chromatogr. A 2010, 1217, 5261-5273.

[10] Ragonese, C., Sciarrone, D., Tranchida, P. O., Dugo, P, Mondello, L., J. Chromatogr. A 2012, 1255, 130-144.

[11] Zeng, A. X., Chin, S-T., Nolvachai, Y., Kulsing, C., Sidisky, L. M., Marriott, P. J., Anal. Chim. Acta 2013, 803, 166-173.

[12] Weber, B. M., Harynuk, J. J., J. Chromatogr. A 2013, 1271, 170-175.

[13] Armstrong, D. W., He, L., Liu, Y.-S., Anal. Chem. 1999, 71 , 3873-3876.

[14] Poole, C. F., Poole, S. K., J. Sep. Sci. 2011, 34, 880-900.

[15] Ragonese, C., Sciarrone, D., Tranchida, P. Q., Dugo, P. Dugo, G., Mondello, L., Anal. Chem. 2011, 83, 7947-7954.

[16] Qi, M., Armstrong, D. W., Anal. Bioanal. Chem. 2007, 388, 889-899.

[17] Ragonese, C., Tranchida, P. Q., Sciarrone, D., Mondello, L., J. Chromatogr. A 2009, 1216, 8992-8997.

[18] Ragonese, C., Tranchida, P. O., Dugo, P., Dugo, G. Sidisky, L. M., Robillard, M. V., Mondello, L., Anal. Chem. 2009, 81, 5561-5568.

[19] Gu, Q., David, F., Lynen, F., Vanormelingen, P., Vyverman W., Rumpel, K., Xu, G., Sandra, P., J. Chromatogr. A 2011 $1218,3056-3063$.

[20] Domínguez, C., Reyes-Contreras, C., Bayona, J. M., J. Chromatogr. A 2012, 1230, 117-122.

[21] Reyes-Contreras, C., Domínguez, C., Bayona, J. M., J. Chromatogr. A 2012, 1261, 164-170.

[22] García Pinto, C., Pérez Antón, A., Pérez Pavón, J. L., Moreno Cordero, B., J. Chromatogr. A 2012, 1260, 200205.

[23] Zapadlo, M., Krupcík, J., Májek, P., Armstrong, D. W. Sandra, P., J. Chromatogr. A 2010, 1217, 5859-5867.

[24] Zapadlo, M., Krupcík, J., Kovalczuk, T., Májek, P., Spánik, I., Armstrong, D. W., Sandra, P., J. Chromatogr. A 2011 1218, 746-751.

25] Purcaro, G., Quinto Tranchida, P., Ragonese, C., Conte, L., Dugo, P., Dugo, G., Mondello, L., Anal. Chem. 2010, 82, 8583-8590.

[26] Mahé, L., Dutriez, T., Courtiade, M., Thiébaut, D., Duot, H., Bertoncini, F., J. Chromatogr. A 2011, 1218 534-544.

[27] IPCS, Chlorobenzenes Other than Hexachlorobenzene: Environmental Aspects, World Health Organization, In ternational Programme on Chemical Safety (CICADs 60), Geneva 2004.

[28] Hu, H., Sun, X., Zhong, Z., Chen, X., Zhang, X., Guo, Y., J. Sep. Sci. 2012, 35, 2922-2928.

[29] Wang, M.-J., McGrath, S. P., Jones, K. C., Environ. Sci. Technol. 1995, 29, 356-362.

[30] Wang, M.-J., Jones, K. C., Chemosphere 1994, 28(7), 1325-1360. 
[31] García Pinto, C., Fernández Laespada, M. E., Herrero Martín, S., Casas Ferreira, A. M., Pérez Pavón, J. L., Moreno Cordero, B., Talanta 2010, 81, 385-391.

[32] Poole, C. F., The Essence of Chromatography, 1st Edition, Elsevier, Amsterdam, 2003.

[33] McNair, H. M., Miller, J. M., Basic Gas Chromatography, 2nd Edition, John Wiley \& Sons, New York, 2009.

[34] Wolska, L., Konieczka, P., Jastrzebska, A., Namieśnik, J., J. Chromatogr. Sci. 2003, 41, 53-56.

[35] Kozani, R. R., Assadi, Y., Sheminari, F., Hosseini, M.-R. M., Jamali, M. R., Talanta 2007, 72, 387-393.

[36] Rouvière, F., Buleté, A., Cren-Olivé, C., Arnaudguilhem, C., Talanta 2012, 93, 336-344.

[37] Grueiro Noche, G., Fernández Laespada, M. E., Pérez Pavón, J. L., Moreno Cordero, B., Muniategui Lorenzo, S., Anan. Bional. Chem. 2013, 405, 6739-6748.
[38] Wang, Y Lee, H. K J Chromatogr. A 1998, 803, 219-225

[39] He, Y., Wang, Y., Lee, H. K., J. Chromatogr. A 2000, 874 149-154.

[40] Vidal, L., Canals, A., Kalogerakis, N., Psillakis, E., J. Chromatogr. A 2005, 1089, 25-30.

[41] Herrero Martín, S., García Pinto, C., Pérez Pavón, J. L., Moreno Cordero, B., J. Chromatogr. A 2010, 1217, 48834889.

[42] Santos, F. J., Sarrión, M. N., Galceran, M. T., J. Chromatogr. A 1997, 771, 181-189.

[43] Wang, M.-J., Jones, K. C., Chemosphere 1991, 23(5), 677691.

[44] Onuska, F. I., Terry, K. A., Anal. Chem. 1985, 57, 801-805. [45] Li, X., Zeng, Z., Xu, Y., Anal. Bioanal. Chem. 2006, 384, 428-1437. 


\title{
IONIC LIQUID AS STATIONARY PHASES IN GAS CHROMATOGRAPHY:
} DETERMINATION OF CHLOROBENZENES IN SOILS

\author{
Rosa María González Paredes, Carmelo García Pinto*, José Luis Pérez Pavón and \\ Bernardo Moreno Cordero
}

\author{
Departamento de Química Analítica, Nutrición y Bromatología. Facultad de Ciencias \\ Químicas, Universidad de Salamanca. 37008 Salamanca, SPAIN \\ *Corresponding author: (fax)+34-923-294483; (e-mail) cgp@usal.es
}

\author{
SUPPORTING INFORMATION
}


Table S1. Physical and chemical properties of four soils studied.

\begin{tabular}{lc|c|c|c}
\cline { 2 - 5 } & Soil 1 & Soil 2 & Soil 3 & Soil 4 \\
\hline pH & 7.13 & 5.06 & 5.62 & 6.47 \\
\% Organic matter & 0.96 & 2.13 & 0.64 & 2.03 \\
\% Carbon & 0.56 & 1.24 & 0.37 & 1.18 \\
C.E.C.* $\left(\mathbf{c m o l ~ k g}{ }^{-1}\right)$ & 8.34 & 6.84 & 2.94 & 31.54 \\
\hline \% Sand & 66.63 & 39.88 & 90.83 & 39.78 \\
\% Silt & 23.51 & 38.47 & 6.21 & 29.66 \\
\% Clay & 9.86 & 21.65 & 2.95 & 30.56 \\
\hline Texture & Sandy & Loam & Sand & Clay loam \\
\hline
\end{tabular}

*C.E.C.: cation-exchange capacity 
Table S2. Chemical composition of IL columns.

SLB-IL59


Table S3. GC temperature programing in each evaluated column.

\begin{tabular}{cccccccccccccc}
\hline $\begin{array}{c}\text { Stationary } \\
\text { phase }\end{array}$ & $\begin{array}{c}\text { UTL } \\
\text { (a) }\left({ }^{\circ} \mathbf{C}\right)\end{array}$ & Polarity & $\begin{array}{c}\text { Initial } \\
\left({ }^{\circ} \mathbf{C}\right)\end{array}$ & $\begin{array}{c}\text { Hold } \\
\text { time } \\
(\mathbf{m i n})\end{array}$ & $\begin{array}{c}\text { Rate 1 } \\
\left({ }^{\circ} \mathbf{C} / \mathbf{m i n}\right)\end{array}$ & $\begin{array}{c}\text { Temp. 1 } \\
\left({ }^{\circ} \mathbf{C}\right)\end{array}$ & $\begin{array}{c}\text { Hold } \\
\text { time } \\
(\mathbf{m i n})\end{array}$ & $\begin{array}{c}\text { Rate 2 } \\
\left({ }^{\circ} \mathbf{C} / \mathbf{m i n}\right)\end{array}$ & $\begin{array}{c}\text { Temp. 2 } \\
\left({ }^{\circ} \mathbf{C}\right)\end{array}$ & $\begin{array}{c}\text { Hold } \\
\text { time } \\
(\mathbf{m i n})\end{array}$ & $\begin{array}{c}\text { Rate 3 } \\
\left({ }^{\circ} \mathbf{C} / \mathbf{m i n}\right)\end{array}$ & $\begin{array}{c}\text { Temp. 3 } \\
\left({ }^{\circ} \mathbf{C}\right)\end{array}$ & $\begin{array}{c}\text { Hold } \\
\text { time } \\
(\mathbf{m i n})\end{array}$ \\
\hline SLB - IL59 & 300 & 59 & 40 & 1 & 20 & 140 & -- & 3 & 145 & -- & 100 & 170 & 5 \\
SLB - IL61 & 290 & 61 & 40 & 1 & 30 & 130 & -- & 2 & 145 & -- & 100 & 190 & 5 \\
SLB - IL82 & 270 & 82 & 40 & 1 & 20 & 140 & -- & 3 & 150 & -- & 100 & 170 & 5 \\
SLB - IL100 & 230 & 100 & 40 & 1 & 20 & 110 & -- & 2 & 115 & -- & 100 & 170 & 4 \\
HP5 & 325 & $\mathrm{NA}^{(\mathrm{b})}$ & 40 & 3.25 & 20 & 100 & -- & 65 & 175 & -- & 50 & 240 & 3.05 \\
\hline
\end{tabular}

(a) UTL: upper temperature limit

(b) NA: data not available 
Table S4. Asymmetry factors of the chlorobenzenes analyzed.

\begin{tabular}{lc|c|c|c}
\cline { 2 - 5 } \multicolumn{1}{c}{ Analyte } & \multicolumn{4}{c}{ Asymmetry Factor (AF) } \\
\cline { 2 - 5 } & SLB-IL59 & SLB-IL61 & SLB-IL82 & SLB-IL100 \\
\hline $\mathbf{1 , 3 - D C B ~}$ & 0.919 & 0.866 & 0.966 & 0.644 \\
\cline { 2 - 5 } $\mathbf{1 , 4 - D C B}$ & \multirow{2}{*}{$\begin{array}{l}\text { S.116 } \\
\mathbf{1 , 3 , 5 - T C B}\end{array}$} & 1.682 & 4.405 & 0.994 \\
\cline { 2 - 5 } $\mathbf{1 , 2 - D C B}$ & 0.934 & 0.48 & & \\
\cline { 2 - 5 } $\mathbf{1 , 2 , 4 - T C B}$ & 1.133 & 0.855 & 1.324 & 0.734 \\
\cline { 2 - 5 } $\mathbf{1 , 2 , 3 , 5 - T e C B}$ & 0.961 & 0.815 & 0.954 & 1.005 \\
\cline { 2 - 5 } $\mathbf{1 , 2 , 4 , 5 - T e C B}$ & 1.082 & 0.82 & 0.918 & \\
$\mathbf{1 , 2 , 3 - T C B}$ & 1.454 & 0.847 & 1.949 & 0.803 \\
$\mathbf{1 , 2 , 3 , 4 - T e C B}$ & 0.953 & 0.725 & 0.989 & 0.835 \\
\cline { 2 - 5 } PeCB & 1.048 & 0.605 & 1.153 & 0.877 \\
HCB & 0.921 & 0.758 & 1.023 & 0.856 \\
\hline
\end{tabular}


Table S5. Calculated and critical values of $t$ for evaluation of matrix effect.

\begin{tabular}{|c|c|c|c|c|c|c|c|c|c|c|c|c|}
\hline \multirow[b]{2}{*}{ Analyte } & \multicolumn{2}{|c|}{ Soils 1 y 2} & \multicolumn{2}{|c|}{ Soils 1 y 3} & \multicolumn{2}{|c|}{ Soils 1 y 4} & \multicolumn{2}{|c|}{ Soils 2 y 3} & \multicolumn{2}{|c|}{ Soils 2 y 4} & \multicolumn{2}{|c|}{ Soils 3 y 4} \\
\hline & $|t|_{\text {calc. }}$ & $t_{\text {critical }}$ & $|t|_{\text {calc. }}$ & $t_{\text {critical }}$ & $|t|_{\text {calc. }}$ & $t_{\text {critical }}$ & $|t|_{\text {calc. }}$ & $t_{\text {critical }}$ & $|t|_{\text {calc. }}$ & $t_{\text {critical }}$ & $|t|_{\text {calc. }}$ & $t_{\text {critical }}$ \\
\hline 1,3-DCB & 57,03 & 2,02 & 0,12 & 2,02 & 82,04 & 2,02 & 51,55 & 2,02 & 11,45 & 2,02 & 70,98 & 2,03 \\
\hline $\begin{array}{l}1,4-\mathrm{DCB} \\
1,3,5-\mathrm{TCB}\end{array}$ & 21,84 & 2,02 & 18,02 & 2,01 & 29,29 & 2,05 & 42,91 & 2,02 & 4,74 & 2,05 & 54,59 & 2,04 \\
\hline 1,2-DCB & 258,00 & 2,09 & 152,94 & 2,06 & 233,69 & 2,06 & 44,29 & 2,02 & 21,77 & 2,03 & 25,65 & 2,02 \\
\hline 1,2,4-ТCВ & 159,31 & 2,07 & 176,46 & 2,07 & 151,23 & 2,07 & 45,83 & 2,02 & 19,78 & 2,01 & 63,55 & 2,01 \\
\hline 1,2,3,5-ТеСВ & 1,75 & 2,05 & 5,30 & 2,04 & 1,07 & 2,02 & 2,02 & 2,03 & 0,74 & 2,02 & 3,27 & 2,02 \\
\hline $1,2,4,5-\mathrm{TeCB}$ & 22,30 & 2,02 & 0,31 & 2,02 & 24,63 & 2,03 & 25,15 & 2,03 & 1,41 & 2,04 & 29,40 & 2,02 \\
\hline 1,2,3-ТСВ & 53,54 & 2,02 & 35,50 & 2,02 & 121,06 & 2,04 & 26,70 & 2,02 & 7,37 & 2,04 & 58,15 & 2,05 \\
\hline 1,2,3,4-ТеCВ & - & - & - & - & - & - & 34,89 & 2,04 & 2,90 & 2,03 & 23,45 & 2,06 \\
\hline PeCB & $\cdots$ & - & - & $\cdots$ & - & - & 27,22 & 2,03 & 10,31 & 2,03 & 25,21 & 2,02 \\
\hline НСВ & 8,01 & 2,05 & 12,83 & 2,05 & 9,19 & 2,06 & 10,28 & 2,05 & 9,58 & 2,05 & 5,52 & 2,02 \\
\hline
\end{tabular}




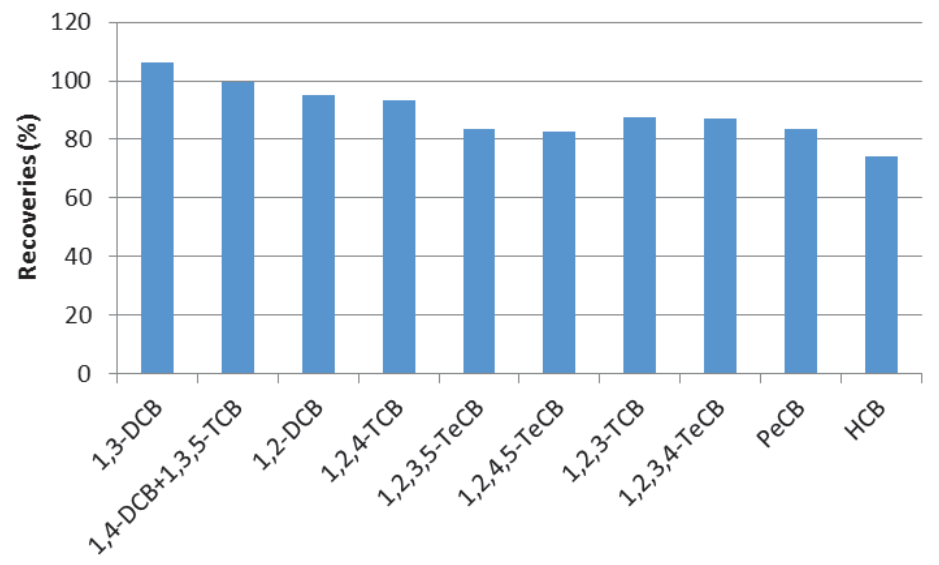

Figure S1. Recoveries obtained on storing the soil at $4{ }^{\circ} \mathrm{C}$ for 5 days, then eliminating the solvent and extracting 7 day after spiking with $1 \mathrm{~g}$ of $\mathrm{MgSO}_{4}$. 


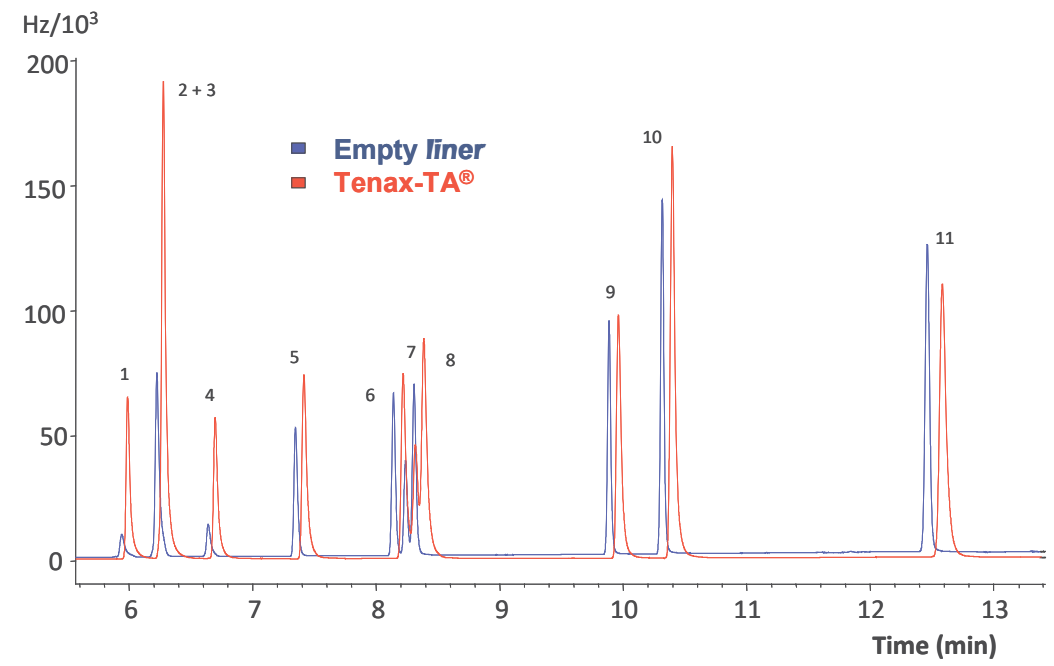

Figure S2. GC- $\mu \mathrm{ECD}$ chromatograms of a standard solution of the target compounds with a liner packed with Tenax-TA ${ }^{\circledR}$ and with an empty liner. 1. 1,3-DCB; 2. 1,4-DCB; 3. 1,3,5-TCB; 4. 1,2-DCB; 5. 1,2,4-TCB; 6. 1,2,3,5-TeCB; 7. 1,2,4,5-TeCB; 8. 1,2,3-TCB; 9. 1,2,3,4-TCB; 10. $\mathrm{PeCB} ; 11 . \mathrm{HCB}$. 


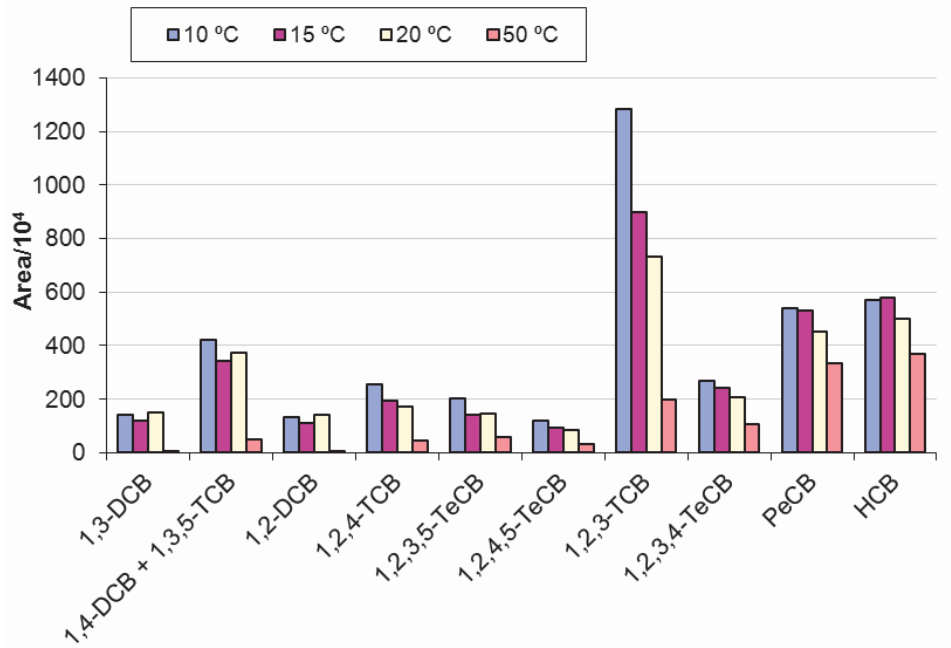

Figure S3. Influence of initial temperature of the liner. 

Derivatización in situ combinada con MEPS para la determinación de clorofenoles en suelos mediante GC-MS 

Artículo de investigación

I.2

Resumen 

Entre los contaminantes orgánicos clorados, los clorofenoles forman un grupo de compuestos químicos ampliamente empleados como biocidas, conservantes de la madera y pesticidas. Pueden ser liberados al medio ambiente durante su producción o durante su uso, aunque también se producen durante el blanqueo de la pasta de madera con cloro en la producción de papel. El uso de estos compuestos como conservantes de la madera ha dado lugar a la contaminación del suelo de los alrededores de los aserraderos en los que se utilizan. Debido a sus propiedades físicas y químicas, los clorofenoles tienden a absorberse en materiales sólidos y a acumularse en suelos, sedimentos, lodos y cenizas. La legislación española respecto a la contaminación de suelos ha establecido niveles genéricos de referencia para una lista de sustancias y diferentes usos del suelo, encontrándose en esa lista los clorofenoles.

Son compuestos moderadamente polares que, en cromatografía de gases, tienden a dar picos anchos y con cola, lo que compromete en gran medida la separación cromatográfica, la integración de los picos y la fiabilidad del método si no han sido derivatizados previamente. La derivatización se emplea en muchos métodos analíticos que utilizan la cromatografía de gases como etapa de separación. Con la derivatización de los clorofenoles se aumenta su volatilidad, se mejoran sus características cromatográficas al disminuir su polaridad y se aumenta la sensibilidad para estos analitos. En el caso de los compuestos fenólicos, la reacción más ampliamente utilizada es la acilación, ya que puede realizarse in situ en medios acuosos en tan sólo unos minutos, con altos rendimientos y con bajo 
coste de reactivos. El reactivo más empleado para ello es el anhídrido acético en medio básico.

La derivatización se aplica junto con las técnicas para la extracción de clorofenoles de matrices de suelos, principalmente extracción por ultrasonidos, extracción asistida por microondas, extracción en fase sólida (SPE) y microextracción en fase sólida (SPME). Estas dos últimas técnicas se pueden aplicar después de la extracción de los clorofenoles del suelo con disolventes. La técnica QuEChERS también ha sido aplicada para la extracción de estos compuestos del suelo, realizándose una derivatización posterior de los extractos.

La microextracción con sorbentes empaquetados (MEPS) se basa en la miniaturización de la técnica convencional de SPE, reduciendo tanto el volumen de muestra como el volumen de disolvente orgánico para la elución de los compuestos y, por tanto, el coste de análisis. La pequeña cantidad de sorbente utilizada en los cartuchos (1-4 mg) permite que se puedan usar más de cien veces mediante la optimización de las condiciones de limpieza. Por otra parte, esta técnica puede llevarse a cabo de forma completamente automática y acoplarse en línea con técnicas cromatográficas. Todas estas características hacen que MEPS sea una buena opción para la preconcentración de los clorofenoles.

El objetivo principal de este trabajo era desarrollar y proponer un nuevo método analítico para la determinación de clorofenoles en suelos basado en el acoplamiento de la derivatización y extracción in situ de los compuestos en el suelo con la preconcentración mediante MEPS automático. Además, también se pretendía comparar MEPS con una versión simplificada 
de QuEChERS, realizando en ambos casos la derivatización con anhídrido acético directamente en el suelo y extrayendo del suelo los compuestos ya derivatizados.

En primer lugar, se realizó un estudio del medio necesario para llevar a cabo la derivatización, probándose 0.4 y $0.1 \mathrm{M}$ de $\mathrm{Na}_{2} \mathrm{HPO}_{4}$ y $0.4 \mathrm{M}$ de $\mathrm{KHCO}_{3}$. Las máximas señales se obtuvieron con $\mathrm{KHCO}_{3}$, lo que indica que un $\mathrm{pH}$ de 8.35 es suficiente para que la reacción tenga lugar. El pH final que proporciona el $\mathrm{Na}_{2} \mathrm{HPO}_{4}$ (9.75) provocaba una disminución de las señales, lo que puede ser debido a la hidrólisis de los compuestos derivatizados.

Para la optimización de las condiciones de MEPS, se realizó primero la comparación de tres sorbentes con los que se cubría un amplio rango de polaridad. $\mathrm{El} \mathrm{C}_{18}$ es un sorbente enlazado que retiene compuestos no polares, el PGC (porous graphitic carbon) es un sorbente de carbono grafitizado poroso que retiene compuestos altamente polares y el PEP (polar enhanced polymer) es un sorbente de poliestireno-divinilbenceno altamente poroso modificado con grupos funcionales de urea que retiene tanto compuestos polares como no polares. Las mayores señales para los analitos de estudio se obtuvieron con el cartucho $\mathrm{C}_{18}$. A continuación se probaron diferentes disolventes de elución: acetato de etilo, metanol y metil tert-butil éter. El acetato de etilo proporcionaba las mayores señales para la primera elución. Además, el porcentaje obtenido con acetato de etilo decrecía de forma sustancial para la segunda y tercera elución, indicando que este disolvente puede ser considerado adecuado para la desorción de los clorofenoles derivatizados del cartucho $\mathrm{C}_{18}$. 
En el MEPS automático, el volumen eluido del cartucho es el volumen inyectado directamente en el inyector y es, por tanto, una importante variable a optimizar. Debido a que el PTV operaba en el modo solvent vent, se estudiaron volúmenes de 10,15 y $20 \mu \mathrm{L}$ para eluir los compuestos retenidos. Con $20 \mu \mathrm{L}$ se obtenía una mala morfología de pico, mientras que para 10 y $15 \mu \mathrm{L}$ se obtuvieron señales similares en cuanto a morfología y área. Se seleccionó, por tanto, $10 \mu \mathrm{L}$, debido a que volúmenes mayores no mejoraban la respuesta, indicando que los compuestos retenidos se eluían adecuadamente del cartucho con el más pequeño.

Para asegurar la eliminación de las pequeñas cantidades de analitos que permanecen en el cartucho y eliminar problemas derivados del efecto memoria, se estudiaron diferentes procedimientos de lavado, seleccionándose la opción en la que se lava dos veces con $100 \mu \mathrm{L}$ de acetato de etilo y dos veces con $100 \mu \mathrm{L}$ de agua ultrapura.

Un importante factor a tener en cuenta es la vida útil del cartucho del MEPS. En este trabajo, todos los estudios se realizaron con el mismo cartucho $\mathrm{C}_{18}$ (más de 300 inyecciones) sin llegar a observarse problemas de reproducibilidad en el rendimiento del dispositivo.

En cuanto al sistema cromatográfico, se optimizaron las variables del modo solvent vent del PTV como temperatura, flujo y tiempo de venteo, seleccionándose, finalmente, $20{ }^{\circ} \mathrm{C}, \quad 50 \mathrm{~mL} \min ^{-1}$ y 0.75 min, respectivamente.

Para evaluar la preconcentración que se lleva a cabo con MEPS, se compararon las señales obtenidas con MEPS y QuEChERS. El cálculo de las 
recuperaciones absolutas no resulta una tarea fácil, ya que sería necesaria la síntesis y purificación de los compuestos derivatizados de los clorofenoles. Sin embargo, el rendimiento de los procedimientos se puede evaluar por comparación de algunos conjuntos de señales. Se toman como referencia las señales obtenidas con una disolución estándar acuosa de los compuestos después de su derivatización con anhídrido acético y extracción con acetato de etilo. Comparadas con esta referencia, las señales obtenidas con el procedimiento QuEChERS en suelos eran del 96\% para 2-clorofenol, 87\% para 4-cloro-3-metilfenol, 90\% para 2,4-diclorofenol y $85 \%$ para 2,4,6triclorofenol. Respecto al procedimiento de MEPS, las señales obtenidas eran entre seis y once veces mayores que las obtenidas con QuEChERS pero, teniendo en cuenta la proporción entre el volumen de muestra y el volumen eluido, las recuperaciones para MEPS parecen encontrarse entre 13 y 23\%. El comportamiento lineal de las señales con la concentración indica que estas recuperaciones son constantes y reproducibles.

La evaluación del efecto de matriz se llevó a cabo con dos tipos de suelos diferentes dopados a seis niveles de concentración, sometidos al proceso de derivatización y extracción in situ y preconcentración propuesto. Las pendientes de las rectas de calibración se compararon mediante un contraste de significación $t$ de Student para determinar si las pendientes eran significativamente diferentes. Este test reveló la existencia de efecto de matriz, por lo que se propuso el método de adición estándar para la correcta cuantificación de los clorofenoles en suelos.

Las características analíticas se determinaron en un suelo dopado con todos los clorofenoles a seis niveles de concentración. Todas las rectas de 
calibración exhibían comportamiento lineal, buenos valores del coeficiente de determinación y ninguna de ellas mostraba fallo de ajuste. Los valores de repetibilidad instrumental y reproducibilidad eran inferiores en todos los casos al 10\% y los límites de detección (LOD) y cuantificación (LOQ) se encontraban entre 0.118 y $0.894 \mu \mathrm{gg}^{-1}$ y entre 0.353 y $2.683 \mu \mathrm{g} \mathrm{kg}{ }^{-1}$, respectivamente.

La validación del método optimizado basado en MEPS se llevó a cabo mediante el análisis de un material de referencia certificado con el método de adición estándar. En todos los casos, las concentraciones calculadas estaban dentro del intervalo de predicción especificado en el material de referencia y eran significativamente iguales al valor de referencia, lo que revela la validez de la metodología propuesta.

En conclusión, en este trabajo se describe por primera vez el acoplamiento de la extracción y derivatización in situ de los clorofenoles en el suelo con la preconcentración mediante MEPS automático. La acilación de los clorofenoles con anhídrido acético en medio acuoso es una gran ventaja cuando seguidamente se utiliza MEPS. Además, la utilización del MEPS automático acoplado en línea con GC permite automatizar el proceso, lo que reduce los errores asociados con el pretratamiento de muestra junto con un considerable ahorro de tiempo de análisis. La comparación de MEPS con la versión simplificada de QuEChERS muestra la preconcentración que se lleva a cabo con MEPS. Por otro lado, los límites de detección obtenidos con el método propuesto que lleva a cabo la preconcentración de los compuestos mediante MEPS (0.118-0.894 $\left.\mu \mathrm{g} \mathrm{kg}^{-1}\right)$ son mucho más bajos que los niveles genéricos de referencia establecidos por la legislación española para los suelos 
contaminados, poniendo de manifiesto la aplicabilidad del método propuesto para el análisis de clorofenoles en suelos. 



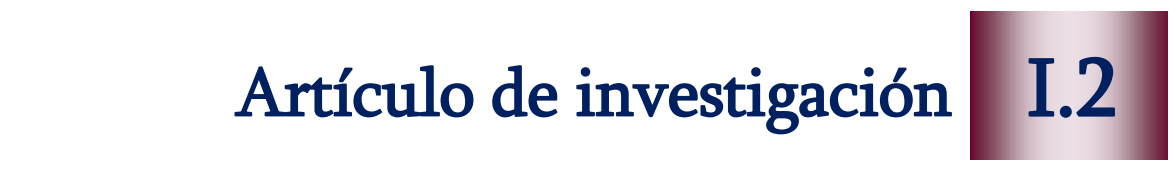

Journal of Chromatography A 1359 (2014) 52-59 

Journal of Chromatography A, 1359 (2014) 52-59

\begin{tabular}{|c|c|c|}
\hline & Contents lists available at ScienceDirect & 涪 \\
\hline & Journal of Chromatography A & 豆 \\
\hline$\overline{\text { ELSEVIER }}$ & journal homepage: www.elsevier.com/locate/chroma & $\Longrightarrow$ \\
\hline
\end{tabular}

In situ derivatization combined to automated microextraction by packed sorbents for the determination of chlorophenols in soil samples by gas chromatography mass spectrometry

Rosa María González Paredes, Carmelo García Pinto*,

José Luis Pérez Pavón, Bernardo Moreno Cordero

Departamento de Química Analítica, Nutrición y Bromatología, Facultad de Ciencias Químicas, Universidad de Salamanca, 37008 Salamanca, Spain

\begin{tabular}{l} 
A R T I C L E I N F O \\
\hline Article history: \\
Received 30 April 2014 \\
Received in revised form 1 July 2014 \\
Accepted 16 July 2014 \\
Available online 24 July 2014 \\
\hline Keywords: \\
In situ derivatization \\
Microextraction by packed sorbents (MEPS) \\
Quick, Easy, Cheap, Effective, Rugged and \\
Safe (QuEChERS) \\
Chlorophenols \\
Soil samples
\end{tabular}

A R T I C L E I N F O

Received 30 April 2014

vised form 1 July 2014

\section{Keywords:}

Microextracti Safe (QuEChERS)

Soil samples

\begin{abstract}
A B S T R A C T
A method based on the coupling of in situ extraction and derivatization of chlorophenols ( $\mathrm{CPs}$ ) (2-chlorophenol, 4-chloro-3-methylphenol, 2,4-dichlorophenol, and 2,4,6-trichlorophenol) from soils, accomplishing their preconcentration by means of automated microextraction by packed sorbent (MEPS) is proposed. After extraction and acylation of the chlorophenols in aqueous medium, the liquid phase obtained is subjected to the MEPS procedure. The QuEChERS (Quick, Easy, Cheap, Effective, Rugged and Safe) and MEPS techniques were compared and the results confirmed the preconcentration carried out with MEPS.

The existence of a matrix effect was checked and the analytical characteristics of the method were determined in a soil sample. The method provided good linearity (from 1 to $12 \mu \mathrm{g} \mathrm{kg}^{-1}$ ), together with good repeatability and reproducibility values (RSD equal to or less than $10 \%$ ). The limits of detection were in the $0.118-0.894 \mu \mathrm{g} \mathrm{kg}^{-1}$ range. A certified reference material was applied to validate the proposed methodology.

(c) 2014 Elsevier B.V. All rights reserved.
\end{abstract}

\section{Introduction}

Among chloroorganic contaminants, CPs form a group of chemicals widely used as biocides, wood preservative and pesticides. They can enter the environment during their manufacture or during their use. They are also produced during the bleaching of wood pulp with chlorine in paper production. The use of these compounds as with chlorine in paper production. The use of these compounds as a wood preservative has resulted in the soil contamination around
the sawmills where these compounds have been used [1]. Owing
to their physico-chemical properties, CPs tend to sorb onto solid to their physico-chemical properties, CPs tend to sorb onto solid material and accumulate in soils, sediments, sludge and ash samples [2]. Spanish legislation regarding soil pollution has established Generic Reference Levels (GRL) for a list of substances and different soil uses. The classification of soils as polluted or non-p
is based on these GRL and takes soil use into account $[3,4]$.

CPs are moderately polar compounds that tend to give broad, tailed peaks, largely compromising chromatographic separation, peak integration, and method reliability if they have not been derivatized previously [5]. Analyte derivatization is employed in

* Corresponding author. Fax: +34 923294483. many analytical methods that employ gas chromatography (GC) as the separation step. Derivatization increases analyte volatility, improves the chromatographic characteristics of an analyte by decreasing its polarity, and/or increases the detector sensitivity of the target analytes [6]. In the case of phenolic compounds, the reaction most widely used is acylation, since this can be carthe reaction most widely used is acylation, since this can be car-
ried out in situ in aqueous medium in just a few minutes, with high yield and low reagent cost [7]. The reagent most widely employed is acetic anhydride in basic medium, fixed with sodium or potassium carbonate [8-10] or sodium or potassium hydrogen carbonate $[5,11,12]$. Other alternatives to the basic medium used are sodium or potassium hydrogen phosphate [13-15] or sodium hydroxide [16].

Derivatization is applied together with the techniques used to extract CPs from soil matrices: mainly ultrasonic extraction [17-19], microwave (MW) extraction [20-22], steam distillation extraction (SDE) [23], solid phase extraction (SPE) [24], and solid phase microextraction (SPME) [25-27]. The latter two techniques can be used after extraction with solvents. QuEChERS has also been used to extract CPs from soil and further derivatization of the extracts [28]. In soils, a simplified version of the original QuEChERS method for the extraction of chlorinated compounds has been developed [29]. This version eliminates the dispersive solid-phase 
extraction (d-SPE) cleanup step after extraction owing to the nonfatty characteristics of soil matrices and hence involves fewer sample treatment steps.

Microextraction by Packed Sorbent (MEPS) is a technique for (ample prepation introduced by Abdel-Rehim in 2004 [30] and is based on a miniaturization of conventional solid-phase extraction (SPE) in which a sorbent packing material is placed between the barrel and needle of a syringe as a cartridge. In comparison with the conventional SPE method this technique reduces both sample volume and that additionally, analysis costs are lower than with SPE. Moreover, the small amount of sorbent used in MEPS cartridges (1-4 mg) enables them to be reused more than 100 times by optimizing the cleaning conditions [31] The MEPS techniquecan be completely automated, conditions . MEPS applications include the analysis of diverse compounds in biological matrices, such as human plasma, blood, urine and saliva $[32,33]$, the analysis of environmental samples, mainly water and wastewater [33-40], and the analysis of food samples [41,42].

Here we propose a new analytical method based on the coupling of the in situ extraction and derivatization of chlorophenols from soils with the preconcentration of the compounds by means of online MEPS. A comparison of MEPS with simplified QuFChERS was carried out The acylation is preferred for the derivatization of CPs present in water solutions [7]. Moreover, the simplicity in coupling MEPS with GC separation facilitates the automation of the procedure. In both cases, derivatization was carried out directly in soi and the derivatized compounds were extracted. In both procedures, a programed temperature vaporizer (PTV) operating in solventvent mode is used to inject the extracts in the gas chromatographic system. The existence of a matrix effect was checked with two types of soils and the analyticatcharateristics of the method were deterof soils (CRM) was analyzed to validate the proposed methodology.

\section{Experimental}

2.1. Chemicals

The chlorophenols employed (2-chlorophenol, 2,4dichlorophenol, 4-chloro-3-methylphenol, 2,4,6-trichlorophenol ) were supplied by Sigma-Aldrich (Steinheim, Germany). Ethyl acetate was HPLC grade (CHROMASOLV ${ }^{\circledR}$ Plus) and was purchased from Sigma-Aldrich (Steinheim, Germany). Methanol was (Dic anhy dride, potassium hydrogen carbonate and dehydrated disodium hydrogen phosphate were from Scharlau (Barcelona, Spain). The ultrapure water used was obtained with an Elgastat UHQ water purification system. Abbreviation of the acetylated derivatives are as follow: 2-chlorophenol (2-CPA); 4-chloro-e-methylphenol (4C-3MPA); 2,4-dichlorophenol (2,4-DCPA); 2,4,6-trichlorophenol (2,4,6-TCPA). The CRM employed was a clay soil (RTC-CRM136) and purchased from LGC Promochem (Barcelona, Spain).

\subsection{Standard solutions and samples}

A stock standard solution of each chlorophenol $\left(2000 \mathrm{mg} \mathrm{L}^{-1}\right)$ was prepared in methanol. These were kept in a refrigerator at $4^{\circ} \mathrm{C}$ and diluted to the desired concentration when used. These solutions were used to optimize the method, to perform the calibration standards, and to spike the soil samples at different concentrations.

Soil matrices were used for the optimization of the sample pretreatment step to check the existence of a matrix effect and to determine the analytical characteristics of the method. Two different soils from Badajoz (Spain) were used: a sandy soil (soil 1) and
Table

hysical and chemical properties of the two soils studied

\begin{tabular}{lll}
\hline & Soil 1 & Soil 2 \\
\hline pH & 5.62 & 6.47 \\
\% Organic matter & 0.64 & 2.03 \\
\% Carbon & 0.37 & 1.18 \\
C.E.C. $\left(\mathrm{cmol} \mathrm{kg}^{-1}\right)$ & 2.94 & 31.54 \\
\% Sand & 90.83 & 39.78 \\
\% Silt & 6.21 & 29.66 \\
\% Clay & 2.95 & 30.56 \\
Texture & Sand & Clay loam \\
\hline
\end{tabular}

C.E.C.: cation-exchange capacity.

a clay-loam soil (soil 2) taken from agricultural cropland. Table 1 shows the physical and chemical properties of these soils.

The procedure used to spike the samples was as follows: $20 \mathrm{~g}$ of soil was placed in a $100 \mathrm{~mL}$ flask and $1 \mathrm{~mL}$ of a chlorophenol solution in ethyl acetate was added (at a suitable concentration for each case). The flask was sealed hermetically and shaken vigorously for case). The flask was sealed hermetically and shaken vigorously for
15 min to achieve perfect homogenization of the compounds in the $15 \mathrm{~min}$ to achieve perfect homogenization of the compounds in the matrix. The samples were stored in a refrigerator $\left(4^{\circ} \mathrm{C}\right)$ for 5 days to allow the interaction between the compounds and the matrix to to evaporate off $(6 \mathrm{~h})$. Then, the flask was closed and stored at $4{ }^{\circ} \mathrm{C}$ again for a further two days.

\subsection{Apparatus}

MEPS was carried out automatically with an MPS2 Multi Purpose Sampler (Gerstel, Mühlheim an der Ruhr, Germany). The method was fully automated across the whole set of samples. Once they had been placed in the autosampler tray, the different steps of the analysis were performed automatically for each sample. In the optimized method, the analytes were retained in a BIN (Barrel Insert mized method, the analytes were retained in a BIN (Barrel Insert
and Needle) assembly containing a packing of $4 \mathrm{mg}$ of a solidand Needle) assembly containing a packing of $4 \mathrm{mg}$ of a solidphase silica-C18 material (volume $8 \mu \mathrm{L}$, mean particle size $45 \mu \mathrm{m}$,
pore size $60 \AA$ ), inserted into a $100 \mu \mathrm{L}$ gas-tight MEPS syringe. This syringe had a modified front fitting and retaining nut to accommodate the MEPS BIN. The BIN assemblies and MEPS syringe were from SGE Analytical Science (Griesheim, Germany). A VT 32-10 tray, with positions for $10-\mathrm{mL}$ vials, was used. The software used to control sample processing was Maestro, from Gerstel.

Analyses of the chlorophenols were performed on a GC-MS instrument (Agilent Technologies, Santa Clara, CA, USA) consisting of an Agilent $7890 \mathrm{~A}$ series gas chromatograph equipped with a 6890 Agilent Technologies programmed temperature vaporizer (PTV) injector and an Agilent 5975C inert XL MSD. A glass wool liner $(71 \mathrm{~mm} \times 2 \mathrm{~mm})$ was used in the PTV injector. Cooling was accomplished with liquid $\mathrm{CO}_{2}$. Chlorophenols were separated on a DB-VRX column $(20 \mathrm{~m} \times 0.18 \mathrm{~mm} \times 1.00 \mu \mathrm{m})$ (J\&W Scientific, Fol.005\% pure; Air dactor was a quadrupole mass spectrometer. Data acquisition was performed with MSD ChemStation, v. E.02.00.493 software from Agilent Technologies. The compounds were identified using the NIST_98 database (NIST/EPA/NIH Mass Spectral Library, version 2.0) for confirmation.

\subsection{Analytical procedure}

2.4.1. In situ extraction and derivatization reaction

$2.5 \mathrm{~g}$ of soil sample was weighed in a $15-\mathrm{mL}$ glass centrifuge tube with a screw cap, which kept the tube closed for most of the sample preparation step, thus preventing losses of volatile compounds during this stage as much as possible. $6 \mathrm{~mL}$ of $0.4 \mathrm{M}$ potassium hydrogen carbonate was added in order to provide a suitable medium for the derivatization reaction. Then, $120 \mu \mathrm{L}$ of 
acetic anhydride was added as the derivatization reagent and the mixture was shaken for 5 min with a Vortex device. Following this, the vial was opened to remove the $\mathrm{CO}_{2}$ generated during the reaction. The tube was centrifuged at $4500 \mathrm{rpm}$ for $5 \mathrm{~min}$. Finally, the liquid phase was filtered with a $0.45 \mu \mathrm{m}$ polytetrafluoroethylene (PTFE) filter and transferred to an autosampler viat for automated MEPS and subsequent measurement.

\subsubsection{Simplified QuEChERS}

When the simplified QuEChERS method was applied, after the removal of $\mathrm{CO}_{2}$ in the extraction and derivatization step $2.5 \mathrm{~mL}$ of ethyl acetate (extraction solvent) was added and the mixture was shaken again for $1.5 \mathrm{~min}$. Following this, $1 \mathrm{~g}$ of $\mathrm{MgSO}_{4}$ and $0.25 \mathrm{~g}$ of $\mathrm{NaCl}$ (prepared in advance in a closed vial) was added, and the mixture was shaken for $1 \mathrm{~min}$ as fast as possible to prevent the formation of $\mathrm{MgSO}_{4}$ congromerates. The tube was centrifuged at formation of $\mathrm{MgSO}_{4}$ conglomerates. The tube was centrifuged at $4500 \mathrm{rpm}$ for $5 \mathrm{~min}$. Finally, the organic layer was filtered with a
$0.45 \mu \mathrm{m}$ PTFE filter and transferred to an autosampler vial for GC $0.45 \mu \mathrm{m}$ PTFE filter and transferred to an autosampler vial for GC injected directly into the programmed temperature vaporizer of the gas chromatograph at a flow rate of $1 \mu \mathrm{Ls}^{-1}$.

\subsubsection{Automated MEPS}

An extract-discard extraction mode was selected In this mode, new aliquots of sample are drawn successively and then discarded after the corresponding extraction. The optimum conditions were as follows: the sorbent (C18) was first conditioned with $100 \mu \mathrm{L}$ of ethyl acetate and then with $100 \mu \mathrm{L}$ of ultrapure water at a flow of $5 \mu \mathrm{Ls}^{-1}$. Next, the sample $(1 \mathrm{~mL})$ was extracted by drawing it from a vial and discarding to the waste (ten cycles of $100 \mu \mathrm{L}$ at a flow rate of $\left.5 \mu \mathrm{Ls}^{-1}\right)$. Then, the sorbent was washed twice with $r(100 \mu \mathrm{L})$ to remove poss and the cartridge was dried by pumping air through it $(10 \times 100 \mu \mathrm{L})$ at a flow rate of $100 \mu \mathrm{L} \mathrm{s}^{-1}$. The chlorophenols were eluted with $10 \mu \mathrm{L}$ of ethyl acetate, and this volume was injected directly into the programmed temperature vaporizer of the gas chromatograph at a flow rate of $1 \mu \mathrm{Ls}^{-1}$. Finally, after elution the cartridge was washed twice with $100 \mu \mathrm{L}$ of ethyl acetate and twice with $100 \mu \mathrm{L}$ of ultrapure water. This latter process was carried out to wash the sorbent and thereby prevent problems due to a memory effect. The time needed for a MEPS extraction was about 13 min. However, with the automation MEPS, the washing and conditioning of the BIN can be performed while the previous sample is still running.

2.4.4. PTV-GC-MS

A $10-\mu \mathrm{L}$ volume was injected in solvent-vent injection mode. The initial injector temperature was set at $20^{\circ} \mathrm{C}$. Vent flow was adjusted to $50 \mathrm{~mL} \mathrm{~min}{ }^{-1}$ and venting time to $0.75 \mathrm{~min}$. Once venting had finished, the split valve was closed and the liner of the PTV was flash-heated at $720^{\circ} \mathrm{C} \mathrm{min}^{-1}$ up to $250^{\circ} \mathrm{C}$. The analytes were then transferred from the liner to the capillary column $(1.5 \mathrm{~min})$. Then, the split valve was opened and the liner temperature was held at the split valve was opened and the liner temperat
$250^{\circ} \mathrm{C}$ until the end of chromatographic analysis.

Oven temperatures were as follows: $60^{\circ} \mathrm{C}$ for $2 \mathrm{~min}$, heated at $120^{\circ} \mathrm{C} \mathrm{min}^{-1}$ to $70^{\circ} \mathrm{C}$, then increased at $95^{\circ} \mathrm{Cmin}^{-1}$ to $115^{\circ} \mathrm{C}$, heated at $65^{\circ} \mathrm{C} \mathrm{min}^{-1}$ to $175^{\circ} \mathrm{C}$, and finally heated at $45^{\circ} \mathrm{C} \mathrm{min}^{-1}$ to $250^{\circ} \mathrm{C}$. These temperature ramps are the maximum ones permitted by the instrumental configuration employed. This temperature program provided a suitable separation for all the derivatized chlorophenols. The total chromatographic run time was $5.70 \mathrm{~min}$. The detector was a quadrupole mass spectrometer. The ion
source temperature was set at $230^{\circ} \mathrm{C}$, the quadrupole at $150^{\circ} \mathrm{C}$, source temperature was set at $230^{\circ} \mathrm{C}$, the quadrupole at $150^{\circ} \mathrm{C}$, and the transfer line at $275^{\circ} \mathrm{C}$. A solvent delay time of $4.00 \mathrm{~min}$ an ionization voltage of $70 \mathrm{eV}$. The synchronous SIM/scan mode, which allowed the collection of both SIM and full scan data in a

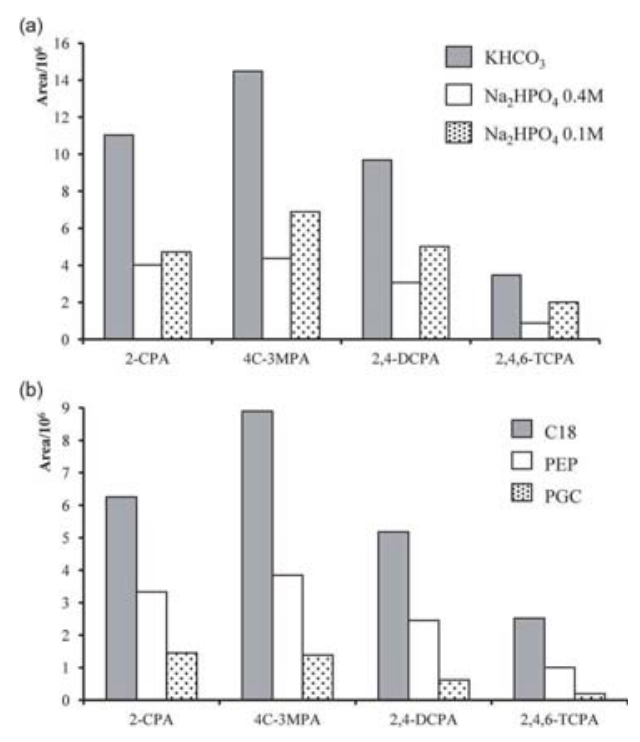
Fig. 1. Comparison of signals obtained (a) with different basic media (b) with three
sorbents in the MEPS BIN.

single run, was used for the optimization studies and the SIM mode for calibration. Full scan $\left(25-350 \mathrm{~m} / \mathrm{z}, 13.73\right.$ scans s$^{-1}$ ) was used for identification and SIM for quantification, selecting the characteristic ions in each case (Table 2). Different dwell times $(1,10,50$ and $100 \mathrm{~ms}$ ) in the SIM mode were assayed. Values of 50 and $100 \mathrm{~ms}$ afforded poor peak definition due to the few points defining it. By contrast, $1 \mathrm{~ms}$ of dwell time increased the noise. Accordingly, a dwell time of $10 \mathrm{~ms}$ was used as a compromise value.

\section{Results and discussion}

3.1. Derivatization reaction

As reported in the Introduction, the acylation of chlorophenols has been used previously for their determination with gas chromatography [6]. One of the important variables affecting the derivatization process is the basic medium used. Here we worked with two different basic media chosen from among those most reported in the literature: a 0.4 and $0.1 \mathrm{M}$ solution of $\mathrm{Na}_{2} \mathrm{HPO}_{4}$ and a $0.4 \mathrm{M}$ solution of $\mathrm{KHCO}_{3}$. For this, a soil spiked at $80 \mu \mathrm{g} \mathrm{kg}-1$ was separated into three portions. Each portion was subjected to the extraction and derivatization process with one of the basic media. Then, the liquid phase obtained from each basic medium was subjected to the MEPS BIN process three times and the extracts were analyzed by GC-MS. The highest signals were obtained for the $0.4 \mathrm{M}$ solution of $\mathrm{KHCO}_{3}$ as shown in Fig. 1a. This indicates that a pH of 8.35 is sufficient for the reaction to take place; the final pH provided for $\mathrm{Na}_{2} \mathrm{HPO}_{4}$ (9.75) elicited a decrease in the signals, which could be due to the hydrolysis of the derivatized compounds [43]. Thus, a basic solution of $0.4 \mathrm{M} \mathrm{KHCO}_{3}$ was selected for further studies. 
Table 2
Analytical characteristics of the method.

\begin{tabular}{|c|c|c|c|c|c|c|c|}
\hline & \multicolumn{2}{|l|}{$m / z$} & \multirow[t]{2}{*}{$R^{2}$} & \multirow{2}{*}{$\begin{array}{l}\text { Instrumental } \\
\text { repeatability (RSD \%) }\end{array}$} & \multirow{2}{*}{$\begin{array}{l}\text { Reproducibility } \\
\text { (RSD \%) }\end{array}$} & \multirow{2}{*}{$\begin{array}{l}\text { LOD } \\
\left(\mu \mathrm{kg}^{-1}\right)\end{array}$} & \multirow{2}{*}{$\begin{array}{l}\text { LOQ } \\
\left(\mu \mathrm{kg}^{-1}\right)\end{array}$} \\
\hline & Quantitation ion & Qualifier ions & & & & & \\
\hline 2-CPA & 128 & 43,170 & 0.9966 & 9.4 & 10 & 0.121 & 0.393 \\
\hline 4C-3MPA & 142 & 43,107 & 0.9931 & 6.6 & 8.1 & 0.118 & 0.353 \\
\hline 2.4-DCPA & 162 & 43,63 & 0.9861 & 6.7 & 7.1 & 0.410 & 1.231 \\
\hline $2,4,6$-TCPA & 196 & 43,198 & 0.9910 & 8.3 & 11 & 0.894 & 2.683 \\
\hline
\end{tabular}

3.2. Optimization of MEPS conditions

We first compared three sorbents in the MEPS BIN C18 (bonded silica sorbent which retains nonpolar compounds), PGC (porous graphitic carbon sorbent which retains highly polar compounds) and PEP (polar enhanced polymer packed with highly porous polystyrene DVB material modified with urea functional groups Dolar an nonpolar compounds). With these three sorbents, a wide range of polarity is covered. C18 was chosen as the optimum sorbent because it provided the highest chromatographic signals for all analytes (Fig. 1b). Different eluting solvents (ethyl acetate, methanol and methyl tert-butyl ether) were evaluated for this solid phase. Thus, the liquid phase obtained from the extraction and derivatization of a soil spiked at $80 \mu \mathrm{g} \mathrm{kg}^{-1}$ was passed through a conditioned MEPS BIN (C18) and subjected to chromatographic separation. For each eluting solvent three portions were tesed and porignals corresponding to the three portions of the elution solvent was assigned $100 \%$. Thus it was possible to compare the different solvents and choose which one provided the highest percentage in the first eluted portion. Fig. 2 shows the percentage obtained for the different eluting solvents for each compound and for the three portions. Ethyl acetate provided higher signals for the first portion than methanol and methyl tert-butyl ether for all the compounds. Moreover, the percentages obtained with ethyl acetate decreased significantly for the secon and this solvent can be considered a suitable solvent for desorption of the derivatized chlorophenols from the C18 BIN.

In automated MEPS, the volume eluted from the BIN is the volume injected directly into the PTV injector and is therefore considered an important variable for optimization. Since the PTV operated in the solvent vent mode, we decided to study elution volumes of 10,15 and $20 \mu \mathrm{L}$ to elute the retained compounds. $15 \mu \mathrm{L}$. With the $20 \mu \mathrm{L}$ volume, a poor peak shape was obtained. In light of these results, a volume of $10 \mu \mathrm{L}$ was selected since at higher volumes the response was not improved, indicating that the retained compounds were adequately desorbed from the BIN with this volume.

To ensure the elimination of the small remaining quantities of the retained analytes (thus avoiding contamination between samples and possible carry-over), the washing step was optimized. Two washing modes were tested: washing once with $100 \mu \mathrm{L}$ of ethyl acetate and once with $100 \mu \mathrm{L}$ of ultrapure water, or washing twice wapure 2.4 and $6.4 \%$, and with the second one it ranged between 0.3 and $0.7 \%$. Accordingly, the optimum washing step involving washing twice with $100 \mu \mathrm{L}$ of ethyl acetate and twice with $100 \mu \mathrm{L}$ of ultrapure water was chosen.

An important factor to be taken into account is the lifetime of the MEPS BIN. Regarding this, all studies of this work were carried out with the same C18 BIN (more than 300 injections) and no wis problems of reproducibility in the performance of the device were

\subsection{Optimization of PTV-GC-MS conditions}

The variables studied in the PTV-GC-MS system were the venting temperature, the venting flow and the venting time for the PTV injector.

The solvent vent injection mode was selected for the PTV injector and the glass wool liner was chosen according to the literature reported for derivatized chlorophenols $[5,44]$. The initial temperature of the liner in which solvent purging occurs, or venting temperature, was studied for values of $15,20,30$ and $50^{\circ} \mathrm{C}$. The areas of the signals decreased gradually with increasing initial temperature, as shown in Fig. 3a. Reproducibility problems were observed for $15^{\circ} \mathrm{C}$, probably due to the difference between the initial temperature of the PTV and the initial oven temperature. Accordingly, we chose a venting temperature of $20^{\circ} \mathrm{C}$. Regarding the purge gas flow, values of $20,40,50$ and $60 \mathrm{~mL} \mathrm{~min}^{-1}$ were studied. Fig. 3b shows the signals obtained with the different flow studied. Fig. 3b shows the signals obtained with the different flow $2,4,6$-trichlorophenol (2,4,6-TCPA). In the case of $60 \mathrm{~mL} \mathrm{~min}^{-1}$ an

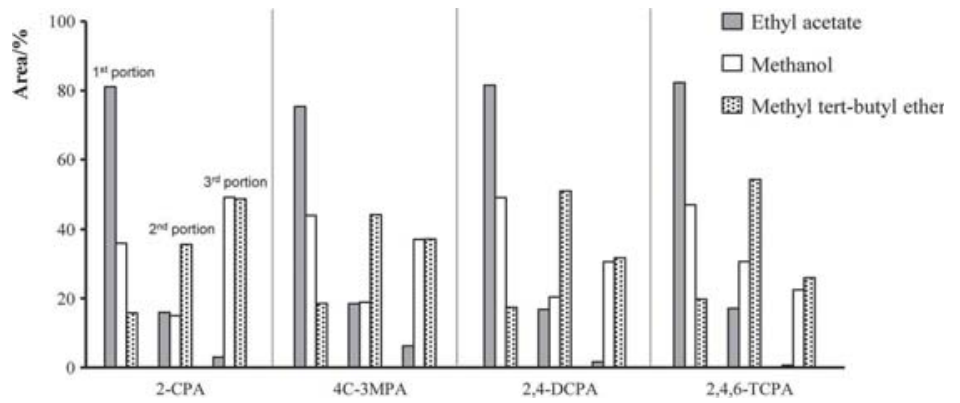

Fig. 2. Desorbed percentage corresponding to the first, second and third portions of the different elution solvents for the target compounds. 
(a)
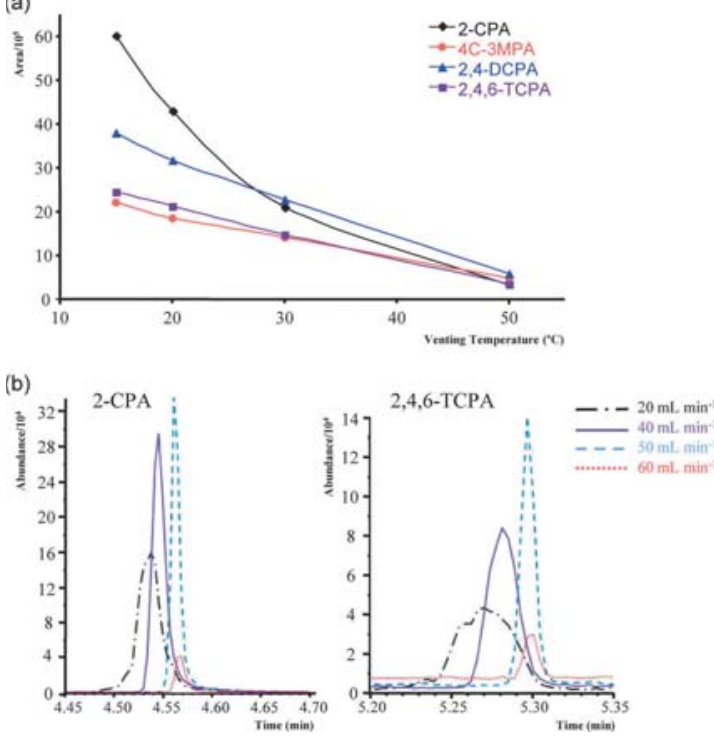

Fig. 3. Influence of (a) the venting temperature and (b) venting flow on the response.

important decrease in the signals occurred whereas in the chromatograms (not shown) the solvent fraction removed did not increase. Flow rates lower than $50 \mathrm{~mL} \mathrm{~min}^{-1}$ produced a poor shape of the peaks. It was therefore decided to work at $50 \mathrm{~mL} \mathrm{~min}^{-1}$, since this permitted the analysis of all the compounds under conditions in which most of the solvent was removed in the venting process. Different venting times $(0.5,0.75$ and $1 \mathrm{~min})$ were assayed. For $1 \mathrm{~min}$, a decrease in the signals occurred since a fraction of the analytes was eliminated with the solvent. With $0.5 \mathrm{~min}$, problems in reproducibility were observed, because the solvent was not completely removed. Maximum signals for all compounds were obtained for $0.75 \mathrm{~min}$ and accordingly this venting time was selected. Fig. 4 shows the extracted ion chromatogram of the soil spiked at $5 \mu \mathrm{g} \mathrm{kg}^{-1}$ under the optimized conditions.

3.4. Comparison QuEChERS and MEPS

To evaluate the preconcentration carried out with MEPS, the signals obtained with the optimized MEPS and with QuEChERS were compared. For this, soil 1 was spiked with chlorophenols (at $80 \mu \mathrm{g} \mathrm{kg}^{-1}$ ). Then, it was divided into two portions of $2.5 \mathrm{~g}$; one portion for in situ extraction and derivatization followed by

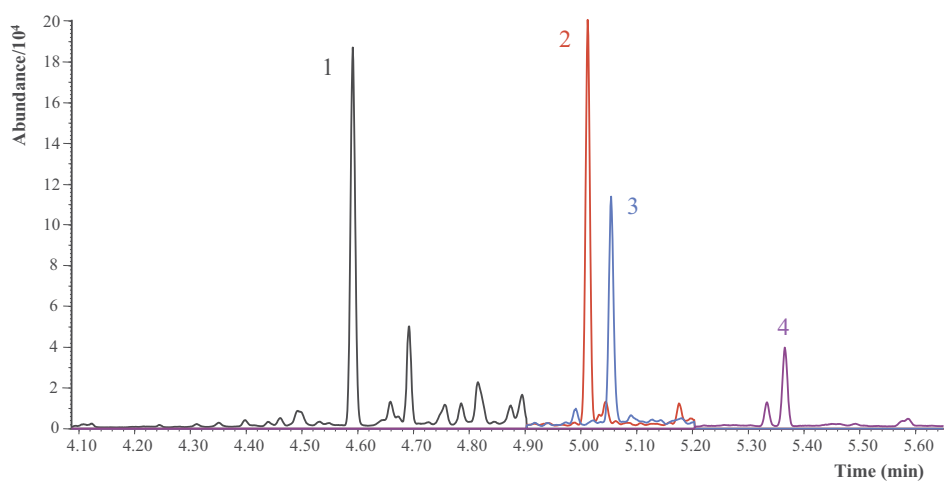

Fig. 4. Chromatogram provided by a soil spiked with all the chlorophenols at $5 \mu \mathrm{gkg}^{-1}$ and subjected to the optimized analytical procedure. 1. 2-CPA; $2.4 \mathrm{C}-3 \mathrm{MPA} ; 3$. 2.4-DCPA: 4.2, 2,6-TCPA. 


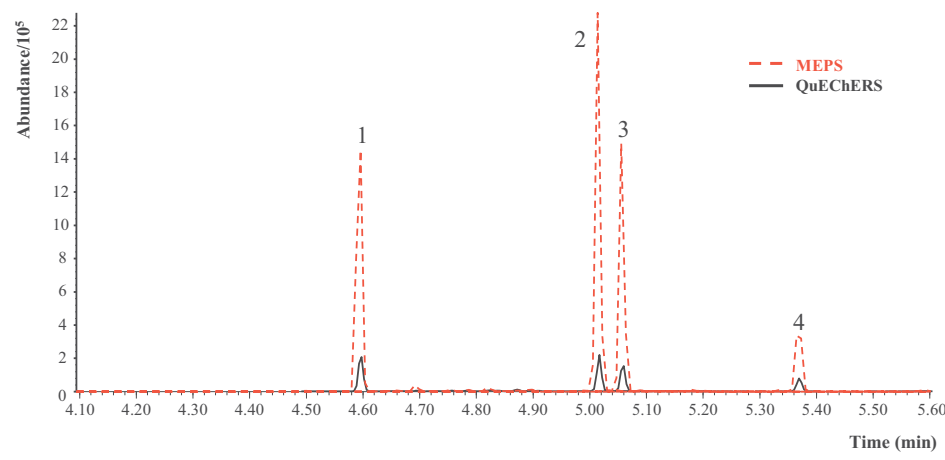

Fig. 5. Comparison of signals obtained with MEPS and QuEChERS. Peak assignments as in Fig. 4.

MEPS, and the other for in situ extraction and derivatization followed by the simplified QuEChERS. Both techniques have been described in the section addressing analytical procedure. The calculation of absolute recoveries is not easy because the synthesis and purification of the derivatives would be necessary. However, the performance of the procedures can be evaluated and compared by comparing some sets of signals. The signals obtained with a standard aqueous solution of the acetylated compounds after the extraction with ethyl acetate are considered the reference. Compared with this reference, the signals obtained with the QuEChERS procedure in soils were $96 \%$ for 2-chlorophenol, $87 \%$ for 4-chloro-3-methylphenol, $90 \%$ for 2,4 dichlorophenol and $85 \%$ for 2,4,6-trichlorophenol. Regarding the MEPS procedure, the signals obtained were between 6- and 11fold higher that those obtained with QuEChERS (Fig. 5) but, ( (sample volume between 13 and 23\%; the linear behavior of the signals with concentration indicate that these recoveries are constant and reproducible.

\subsection{Matrix effect}

The existence of a matrix effect is very common in many analytical techniques used for the determination of chemicals in soils. To evaluate the possible existence of a matrix effect when the proposed procedure was applied to determine chlorophenols in soils, two different soil samples (soil 1 and 2) spiked at six different concentration levels for each compound were extracted and derivatized. Each concentration level was subjected to the MEPS process four times and analyzed with the optimized GC method. The slopes of the calibration curves were compared by Student's t-test to determine whetherthes The calculated value of $t$ for all compounds was higher than the critical value. Thus, there were significant differences in slope for the two matrices considered and the matrix effect was present Moreover, the influence of the matrix was different for each of the

Table 3

\begin{tabular}{|c|c|c|c|c|}
\hline & Compound & Calculated value $\left(\mu \mathrm{g} \mathrm{kg}^{-1}\right)$ & Certified value $\left(\mu \mathrm{g} \mathrm{kg}^{-1}\right)^{*}$ & Prediction interval $\left(\mu \mathrm{g} \mathrm{kg}^{-1}\right)^{*}$ \\
\hline CRM136 & $\begin{array}{l}\text { 2-CP } \\
\text { 2,4-DCP } \\
2,4,6-\mathrm{TCP}\end{array}$ & $\begin{array}{l}(1.0 \pm 0.3) 10^{3} \\
(5 \pm 1) 10^{2} \\
(2.6 \pm 0.6) 10^{3}\end{array}$ & $\begin{array}{l}1200 \pm 154 \\
605 \pm 42.3 \\
3480 \pm 399\end{array}$ & $\begin{array}{l}299-2100 \\
356-854 \\
1140-5820\end{array}$ \\
\hline
\end{tabular}

compounds. These differences could be explained in terms of the different interactions of the compounds in the two types of soils, which have a complex porous structure and contain different proportions of minerals and natural organic components. To overcome this matrix effect, a standard additions protocol was proposed that has already been shown to work properly for soil matrices [24].

3.6. Analytical characteristic of the method

Calibration straight lines were obtained with a soil spiked at six concentration levels ranging from 1 to $12 \mu \mathrm{g} \mathrm{kg}^{-1}$. Each concentration level was subjected to the extraction and derivatization procedure, and each of the liquid phases obtained was subjected to the MEPS process and analyzed four times. All the calibrations exhibited linear behavior, with good values of the coefficient of determination $\left(R^{2}\right)$, as shown in Table 2 . The validity of the models generated was checked using ANOVA, and none of the models generated was found to show lack of fit. The limits of detection (LODs) of the proposed method ranged from to 0.118 and $0.894 \mu \mathrm{g} \mathrm{kg}^{-1}$, whereas the limits of quantification (LOQs) ranged from to 0.353 and $2.683 \mu \mathrm{g} \mathrm{kg}^{-1}$.

Instrumental repeatability and reproducibility were determined at a concentration of $5 \mu \mathrm{g} \mathrm{kg}^{-1}$. Instrumental repeatability was evaluated by subjecting this solution to the MEPS procedure and injecting an extracted and derivatized liquid phase 10 times. The reproducibility of the extraction and derivatization procedure was calculated by injecting 8 different extracts. These extracts were subjected to the MEPS process three times. The values (expressed as RSD) are shown in Table 2 and were highly satisfactory.

\subsection{Method validation}

To validate the optimized method, a certified reference material was analyzed. A standard additions protocol was proposed for the accurate determination of chlorophenols. A six-level calibration study was performed, analyzing four replicates from each level. Table 3 shows the concentration calculated, the certified value

Values provided in the Certificates of Analysis of the CRM soils. 
and the prediction interval for each compound. In most cases, the calculated concentrations were close to the reference values and, in all cases, close to those values within the prediction interval specified in the certified material. This reveals the validity of the methodology proposed for the determination of these compounds in soil matrices.

\section{Conclusions}

A new analytical method based on the coupling of in situ extraction and derivatization of chlorophenols from the soil with preconcentration by means of on-line MEPS has been developed. preconcentration by means of on-line MEPS has been developed. The acylation of CPs with acetic anhydride in aqueous medium is a
great advantage when MEPS is used after it. Furthermore, the cougreat advantage when MEPS is used after it. Furthermore, the cou-
pling of MEPS with GC allows the process to be automated, which reduces the errors associated with sample treatment together with a considerable saving of analysis time. The comparison of MEPS with simplified QuEChERS shows the preconcentration carried out with MEPS.

The analytical characteristics of the method were calculated for a spiked soil sample, with the observation of linear behavior, no lack of fit, suitable reproducibility and instrumental repeatability, and much lower limits of detection $\left(0.118-0.894 \mu \mathrm{g} \mathrm{kg}^{-1}\right)$ than the Generic Reference Levels (GRL) established in the Spanish legislation for soil pollution. The validation of the proposed method was carried out by determining the concentration of chlorophenols in a certified reference material. The calibration strategy used was the standard additions protocol, and the results obtained were highly satisfactory.

\section{Acknowledgments}

Financial support from de DGI (CTQ2010-17514) is acknowledged. R.M.G.P. is grateful for a predoctoral grant from the University of Salamanca. The authors wish to thank the Languages Service of the University of Salamanca for supervising the English version of this manuscript.

\section{References}

[1] ATSDR (1999). Toxicological profile for chlorophenols. U.S. Departament of health and human services, Public Health Service, Agency for Toxic Substances

and Disease Registry.
[2] P. de Morais, T. Stoichev, M.C.P. Basto, M.T.S.D. Vasconcelos, Extraction and preconcentration technicues. Ministerio de la Presidencia (Gobierno de España). Real Decreto 9/2005, Boletín oficial del Estado 14 Enero 2005, Madrid, pp. 1833-1843.

[4] E. Martí,. Sierra, J. Cáliz, G. Montserrat, X. Vila, M.A. Garau R. Cruañas, Ecotoxity of chlorophenolic compounds depending on soil characteristics, Sci. Total. Environ. 409 (2011) 2707-2716.

[5] J.L. Pérez Pavón, A.M. Casas Ferreira, M.E. Fernández Laespada, B. Moreno of programmed temperature vaporizer and an in situ derivaization reaction to improve sensitivity in headspace-gas chromatography. Apllication to the
(2009) $1192-1199$

[6] R.J. Wells, Recent advances in non-silytation derivatization techniques for gas (1999) 1-18.

[7] A.M. Casas Ferreira, M.E. Fernández Laespada, J.L. Pérez Pavón, B. Moreno Cos chromatographic deterivatization as sample preparation technique for

R. Morales, M. Cruz Ortiz, LA. Sarabia, J. Chromatogr. A 1296 (2013) 70-83. factors to determine chlorophenols in water by means of headspace solid-phase microextraction and gas chromatography coupled with mass spectrometry an

[9] F. Galán-Cano, R. Lucena, S. Cárdenas, M. Valcarcel, Ionic liquid based in situ solvent formation microextraction coupled to thermal desorption for chlorophenols determination in waters by gas
trometry, J. Chromatogr. A 1229 (2012) 48-54.

[10] H. Faraji, M.S. Tehrani, S.W. Husain, Pre-concentration of phenolic compounds in water samples by novel liquid-liquid microextraction and determination by gas chrom
[11] M. Llompart, M. Lourido, P. Landin, C. Garcia-Jares, R. Cela, Optimization of a derivatization-solid-phase microextraction method for the analysis of thirty phenolic pollutants in water samples, J. Chromatogr. A 963 (2002) 137-148. 12] E. Baltussen, F. David, extraction-thermal desorption-gas chromatography-mass spectrometry anal-
ysis: Determination of phenols in water samples.. Microcolumn Sep. $11(1999)$ $471-474$

[13] J. Regueiro, E. Becerril, C. Garcia-Jares, M. Llompart, Trace analysis of parabens, triclosan and related chlorophenols in water by headspace solid-phase microextraction with in situ derivatization and gas chromatography-tandem

[14] E. Priego-López, M. de Castro, Ultrasound-Assisted Derivatization of Phenolic compounds in spiked water samples before pervaporation, gas chromatographic separation, and flame ionization detection, Chromatographia 57 (2003)

513-518.
[15] J. Regueiro, M. Llompart, E. Psillakis, J. Garcia-Monteagudo, C. Garcia-Jares, in water Talantasted 79 (2009) 1387 -1397.

16] R. Ito M. Kawago Nakazawa, Hollow-fiber-supported liquid phase microextraction with in situ derivatization and gas chromatography-mass spectrometry for determina-
tion of chlorophenols in human urine samples, J. Chromatogr. B 872 (2008) tion of chlorophenols in human urine samples, J. Chromatogr. B 872 (2008)
$63-67$.

7] J. Llorca-Pórcel, M. Martínez-Parreño, E. Martínez-Soriano, I. Valor, Analysis of means of ultrasonic solvent extraction and stir bar sorptive extraction with in situ derivatization, J. Chromatogr. A 1216 (2009) 5955-5961.

[18] P. Tölgyessy, B. Vrana, M. Bartal, Z. Krascsenits, K. Silhárová, Determination of chlorophenols in sediments usisng ultrasonic solvent extraction followed by 389-392.

19] N. Campillo, N. Aguinaga, P. Viñas, L. López-García, M. Hernández-Córdoba,
Capillary gas chromatography with atomic emission detection for dertemining chlorophenos in water and soil samples. Anal. Chim. Acta 552 (2005)

$182-189$.
[20] R. Ganeshjeevan, R. Chandrasekar, P. Sugumar, P. Kadigachalam, G. Radhakrishnan, Focused microwave aqueous extraction of chlorophenols from solid 1069 (2005) 275-280.

[21] M.C. Alonso, D. Puig, I. Silgoner, M. Grasserbauer, D. Barceló, Determination of priority phenolic comounds in soil samples by various extraction methods followed by liquid chromatography-atmospheric pressure chemical ionisation

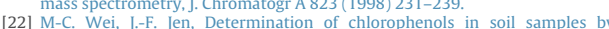
microwave-assisted extraction coupled to headspace solid-phase microextraction and gas chromatography-electron-capture detection, I Chromatogr. A 1012 (2003) 111-118

[23] R. Ganeshjeevan, R. Chandrasekar, P. Kadigachalam, G. Radhakrishnan, Rapid, one-pot derivatization and distillation of chlorophenols from solid samples 4] With their on-line enrichment, J. Chromatogr. A 140 (2007) $168-173$.

24] M.Llompart, B. Blanco, R. Cela, Determination of phenols in soils by in situ acety25-32.

[25] R. Baciocchi, M. Attinà, G. Lombardi, M.R. Boni, Fast determination of phenols in contaminated soils, J. Chromatogr. A 911 (2001) 135-141.

126] M-R. Lee, Y-C. Yeh, W-S- Hsiang, B-H. Hwang, Solid-phase microextraction and gas chromatography-mass spectrometry for determining chlorophenols from 1998) $317-324$

chlorophenols in soils using accelerated solvent extraction combined with solid-phase microextraction, Anal. Chem. 72 (2000) 546-551.

28] J.A. Padilla-Sánchez, P. Plaza-Bolaños, R. Romero-González, A. Garrido-Frenich, J.L. Martínez Vidal, Application of a quick, easy, cheap, effective, rugged and nols, nitrophenols and cresols in agricultural soils, analyzed by using gas chromatography-triple quadrupole-mass spectrometry/mass spectrometry, J.

Chromatogr. A 1217 (2010) 5724-5731.
[29] C. García Pinto, M.E. Fernández Laespada, S. Herrero Martín, A.M. Casas Ferreira, J.L. Pérez Pavón, B. Moreno Cordero, Simplified QuEChERS approach for $385-391$.

[30] M. Abdel-Rehim, New trend in sample preparation: on-line microextrac tion in packed syringe for liquid and gas chromatography apllications. Determiantion of local anaesthetics in human plasma samples using gas

[31] M. Abdel-Rehim, Microextraction by packed sorbent (MEPS): A tutorial, Anal. Chim. Acta 701 (2011) 119-128.

[32] R. Mandrioli, L Mercolini, D. Lateana, G. Boncompagni, M.A. Raggi, Analysis of risperidone and 9-hydroxyrisperidone in human plasma, urine and saliva by

MEPS-LC-UV, J. Chromatogr. B 879 (2011) 167-173.
[33] J. Aufartová, C. Mahugo-Santana, Z. Sosa-Ferrera, J.J. Santana-Rodríguez, L. Nováková, P. Solich, Determination of steroid hormones in biological and environmental samples using green m.
Anal. Chim. Acta 704 (2011) $33-46$.

[34] A. El-Beqqali, A. Kussak, M. Abdel-Rehim, Fast and sensitiv environmen-
tal analysis utilizing microextraction in packed syringe online with gas 
chromatography-mass spectrometry. Determination of polycyclic aromatic

hydrocarbons in water, J. Chromatogr. A 1114 (2006) 234-238.
[35] M. Moeder, S. Schrader, U. Winkler, R. Rodil, At-line microextraction by packed sorbent-gas chromatography-mass spectrometry for the determination of UV (2010) 2925-2932.

[36] A. Prieto, S. Schrader, M. Moeder, Determination of organic priority pollutants and amerging compounds in wastewater and snow samples using multiresidue protocols on the basis of microextraction by packed sorbents coupled to large volume inje tio (2010) $6002-6017$.

[37] 1. González-Mariño, J.B. Quintana, I. Rodríguez, S. Schrader, M. Moeder, Fully automated determination of parabens, triclosan and methyl triclosan in
wastewater by microextraction by packed sorbents and gas chromatographymass spectrometry, Anal. Chim. Acta 684 (2011) 59-66.

[38] A. Prieto, S. Schrader, C. Bauer, M. Moeder, Synthesis of a molecularly imprinted poly (2011) 146-152.

[39] G. Grueiro Noche, M.E.Fernández Laespada,J.L. Pérez Pavón, B. Moreno Cordero, S. Muniategui Lorenzo, Microextraction by packed sorbent for the analyis of pharmaceutical residues in environmental water samples by in situ derivatization-programmed temperature vaporizer-gas chromatographymass spectrometry, J. Chromatogr. A 1218 (2011) 9390-9396.

B. Moreno Cordero, S. Muniategui Lorenzo, Determination of chlorobenzenes in water samples
based on fully automated microextraction by packed sorbent coupled with programmed temperature vaporization-gas chromatography-mass spectrometry. Anal. Bioanal. Chem. 405 (2013) 6739-6748.

[41] J. Gonçalves, B. Mendes, C.L. Silva,,J.S. Câmara, Development of a novel microextractin by packed sorbent-based approach followed by ultrahigh pressure fiquid chromatography as a powerful technique for quantification phenolic $13-23$.

[42] C. García Pinto, A. Pérez Antón, J.L. Pérez Pavón, B. Moreno Codero, Coupling of microextraction by packed sorbents with gas chromatography with ionic liquid stationary phases for the determination of haloanisoles in wines, J. Chromatogr. A $1260(2012) 200-205$

Crey, R.J. Sundberg, Advanced Organic Chemistry. Part A. Structure and Mechanisms, 4th ed., Kluwer Academic/Plenum Publishers, 2000.

[44] J. Benito Quintana, R. Rodil, S. Muniategui Lorenzo, P. López Mahía, D.
Prada Rodríguez, Multiresidue analysis of acidic and polar organic contamiPants in wirguez, Multiresidue analysis of acidic and polar organic contaminants in water samples by stir-bar sorptive extraction-liquid desorption-gas
chromatography-mass spectrometry, J. Chromatogr. A 1174 (2007) 27-39. 


\section{CAPÍTULO II.}

DESARROLLO DE METODOLOGÍAS ANALÍTICAS PARA LA DETERMINACIÓN DE COMPUESTOS DE INTERÉS BIOLÓGICO EN

MUESTRAS DE ORINA 



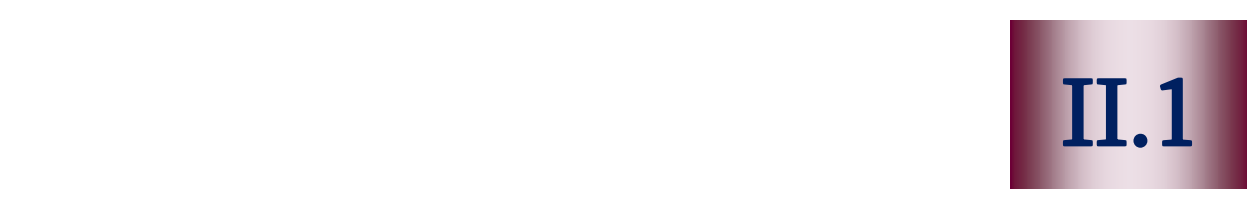

Derivatización acoplada a HS-PTV-GC-MS para la determinación de aminoácidos. Aplicación a muestras de orina 

Artículo de investigación II.1

Resumen 

El perfil metabólico en los fluidos biológicos revela el estado de los procesos biológicos en el cuerpo humano en un determinado momento. Por ello, la metabolómica es una prometedora herramienta para la detección precoz de enfermedades y para una mejor comprensión de los sistemas biológicos. En este contexto, debido a su papel vital en muchas rutas metabólicas, los aminoácidos son uno de los grupos de compuestos más importantes para los estudios de perfil metabólico. Muchas enfermedades y desórdenes metabólicos se pueden diagnosticar por la determinación de los niveles de concentración de aminoácidos. A pesar de que algunos aminoácidos han sido estudiados y propuestos como posibles biomarcadores de cáncer, su validez como tales es incierta todavía y son necesarios más estudios y el análisis de un gran número de muestras. El desarrollo de nuevos métodos analíticos es un punto clave para la investigación y descubrimiento de biomarcadores sensibles, para la validación de los mismos y para una detección temprana de enfermedades.

Para la determinación de aminoácidos se han utilizado varias técnicas analíticas como la electroforesis capilar (capillary electrophoresis, CE) con diferentes sistemas de detección, la cromatografía líquida y la cromatografía de gases. El acoplamiento de la cromatografía de gases con detección mediante espectrometría de masas ha demostrado ser una potente herramienta analítica con muchas ventajas para la identificación y cuantificación simultánea de múltiples compuestos en diferentes matrices biológicas, especialmente cuando se aplica a la investigación de fluidos biológicos humanos. 
Debido a la presencia de grupos altamente polares $\left(-\mathrm{NH}_{2},-\mathrm{COOH}\right)$ y a su naturaleza no volátil, los aminoácidos necesitan ser derivatizados para el análisis por GC-MS. La mayoría de las reacciones de derivatización usan reactivos apolares que requieren disolventes orgánicos y no son compatibles directamente con medios acuosos. Sin embargo, los cloroformiatos se han utilizado ampliamente como reactivos de derivatización de los aminoácidos, con la ventaja de poder llevar a cabo la reacción in situ en medios acuosos a temperatura ambiente. Mientras que el cloroformiato de isobutilo forma un gran número de subproductos y puede dar lugar a algunos compuestos derivatizados no volátiles, el cloroformiato de etilo (ECF) se ha empleado satisfactoriamente para la derivatización de aminoácidos en muestras de orina. No obstante, en estos casos, los compuestos derivatizados se extraen de la mezcla mediante LLE, LLME o SPME antes de ser inyectados en el sistema cromatográfico. El acoplamiento de la derivatización con HS, tal y como se propone en este trabajo, elimina este paso de extracción y hace innecesario el uso de un pretratamiento adicional para aislar los compuestos derivatizados de la matriz. Además, el uso de HS evita las interferencias de los compuestos no volátiles de la matriz. El acoplamiento de la derivatización y HS es, por tanto, una buena alternativa para el análisis de los aminoácidos en diferentes muestras.

El objetivo de este trabajo es desarrollar un nuevo método para la determinación de aminoácidos. La estrategia podría dividirse en dos partes principales. La primera de ellas es un paso manual muy sencillo en la que la muestra y todos los reactivos se añaden al vial para que la reacción de derivatización tenga lugar. La segunda etapa es un procedimiento 
completamente automático en el que se coloca el vial en el automuestrador del equipo y los aminoácidos derivatizados son separados y analizados por HS-PTV-GC-MS. Con la información de que disponemos, es la primera vez que se ha propuesto esta derivatización acoplada al espacio de cabeza para la determinación de estos analitos.

El estudio y optimización de las variables involucradas en cada una de las etapas, así como las características analíticas del método se llevaron a cabo con una disolución acuosa de los aminoácidos sometida a la reacción de derivatización con cloroformiato de etilo, etanol y piridina en medio básico.

Las variables estudiadas para determinar las condiciones óptimas para la derivatización fueron el $\mathrm{pH}$ y el volumen de piridina, etanol y cloroformiato de etilo. Cabe mencionar que el volumen de etanol y ECF se optimizaron simultáneamente para minimizar el número de subproductos formados, es decir, se añadían iguales volúmenes de etanol y de ECF. La eficacia más alta de derivatización se obtuvo con un $\mathrm{pH}$ de 9.0, $100 \mu \mathrm{L}$ de piridina, $120 \mu \mathrm{L}$ de etanol y $120 \mu \mathrm{L}$ de ECF.

Para determinar las condiciones óptimas del espacio de cabeza, se estudiaron la temperatura y el tiempo de generación de espacio de cabeza y el volumen de muestra mediante un diseño experimental multivariante de tipo Box-Behnken, para estudiar posibles interacciones entre ellas. Este diseño permite generar superficies de respuesta usando tres niveles de cada factor estudiado, en este caso: 70, 80 y $90{ }^{\circ} \mathrm{C}$ para la temperatura; 10,20 y 30 min para el tiempo y 1.0, 2.5 y $4.0 \mathrm{~mL}$ para el volumen de muestra. La respuesta seleccionada fue el área de pico correspondiente al pico base del espectro de cada compuesto derivatizado. La temperatura que proporcionaba 
las máximas señales para todos los compuestos era $90{ }^{\circ} \mathrm{C}$. Aunque los resultados obtenidos respecto al tiempo y el volumen de muestra eran menos concluyentes, tiempos cortos y volúmenes pequeños daban lugar a señales con baja reproducibilidad para la mayoría de los casos (entre 17 y 52\%), razón por la que se seleccionaron $30 \mathrm{~min}$ de tiempo y $3 \mathrm{~mL}$ de volumen de muestra. A pesar de que con estos valores no se alcanzaban las máximas señales, éstas eran apropiadas y daban lugar a buenos valores de reproducibilidad (2-12\%) para el proceso completo de derivatización y determinación instrumental.

Las variables estudiadas en el sistema PTV-GC-MS fueron temperatura, flujo y tiempo de venteo para el PTV y tiempo de permanencia (dwell time) para el MS. Finalmente se seleccionaron $70{ }^{\circ} \mathrm{C}$ de temperatura,

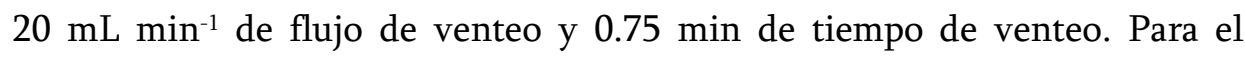
tiempo de permanencia, se eligió $1 \mathrm{~ms}$, ya que proporcionaba la mejor definición de pico sin un aumento significativo del ruido.

Las rectas de calibración para cada compuesto se obtuvieron usando norleucina como patrón interno para compensar las variaciones del proceso completo, y todas ellas mostraron comportamiento lineal con buenos valores de coeficiente de determinación y ninguna de ellas mostraba fallo de ajuste. La repetibilidad instrumental se determinó a dos niveles de concentración, obteniendo valores entre 3.5 y $11.5 \%$ para el nivel bajo y entre 1.6 y $5.9 \%$ para el nivel alto. Los límites de detección (LOD) y cuantificación (LOQ) se encontraban entre 0.15 y $2.89 \mathrm{mg} \mathrm{L} \mathrm{L}^{-1}$ y entre 0.46 y $8.67 \mathrm{mg} \mathrm{L}^{-1}$, respectivamente. 
Para examinar la aplicabilidad del método propuesto a muestras reales de orina y para evaluar el efecto de matriz, se realizó una comparación de las señales obtenidas con una disolución acuosa y tres muestras de orina de voluntarios diferentes. Se observaron diferencias entre la disolución acuosa y las muestras de orina, por lo que se estudió la posibilidad de diluir las muestras de orina con agua, probándose diluciones hasta 1:6. Incluso con esta dilución no se consiguió eliminar el efecto de matriz, por lo que se propuso el método de adición estándar para la determinación de estos analitos en muestras de orina.

Debido a la presencia de efecto de matriz y para simplificar el procedimiento de determinación de los aminoácidos en orina, se llevó a cabo una comparación entre una adición estándar convencional con seis niveles de concentración y una adición estándar de un punto. No se encontraron diferencias significativas en la concentración calculada entre ambos métodos, y por tanto se propuso la adición estándar de un punto para la determinación de estos analitos en orina, ya que esta estrategia hace el procedimiento final más simple y rápido.

El método desarrollado se aplicó al análisis de seis muestras de orina de voluntarios sanos para cuantificar los aminoácidos y estimar la concentración de estos compuestos endógenos en orina. Para ello se realizó una adición estándar con un punto y norleucina como patrón interno. Las concentraciones de los analitos fueron normalizadas a la concentración total de creatinina de cada muestra de orina, observándose niveles de cada aminoácido similares en las seis muestras. 
Como conclusiones podemos mencionar que se ha desarrollado un nuevo método analítico que acopla la derivatización de los aminoácidos a la determinación de los mismos mediante HS-PTV-GC-MS. Además, se han optimizado todas las variables relacionadas con la derivatización, espacio de cabeza y sistema cromatográfico. La derivatización de los analitos con cloroformiato de etilo en medio acuoso supone una gran ventaja cuando se analizan muestras biológicas como es la orina. El acoplamiento de la derivatización con el espacio de cabeza permite la eliminación del uso de disolventes o de otros tratamientos para extraer los compuestos derivatizados de la matriz antes de su inyección en GC. Esta configuración instrumental minimiza la manipulación de la muestra y, por tanto, reduce los errores asociados a esta etapa del análisis y el consumo de disolventes.

Teniendo en cuenta los buenos resultados conseguidos en este trabajo, la metodología propuesta puede ser aplicada en futuros estudios para analizar un gran número de muestras de orina tanto de voluntarios sanos como de pacientes y evaluar las posibles diferencias en el nivel de concentración de estos analitos entre ambos grupos. 


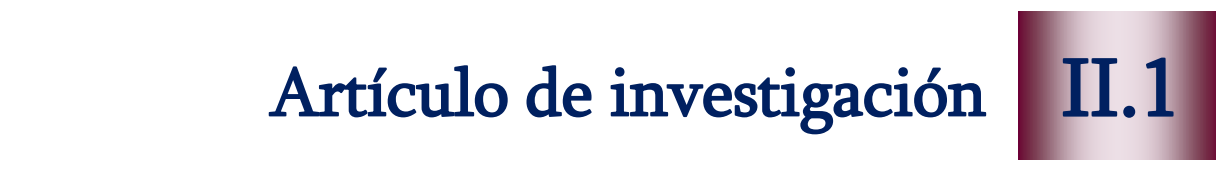

Journal of Separation Science 39 (2016) 3375-3383 

Rosa María González Paredes Carmelo García Pinto José Luis Pérez Pavón Bernardo Moreno Cordero

Departamento de Química Analítica, Nutrición y

Bromatología, Facultad de Ciencias Quimicas, Universidad

Received February 9, 2016 Revised June 23, 2016 Accepted June 24, 2016

\section{Research Article}

Derivatization coupled to headspace programmed-temperature vaporizer gas chromatography with mass spectrometry for the determination of amino acids: Application to urine samples

A new method based on headspace programmed-temperature vaporizer gas chromato raphy with mass spectrometry has been developed and validated for the determination of amino acids (alanine, sarcosine, ethylglycine, valine, leucine, and proline) in human urine samples. Derivatization with ethyl chloroformate was employed successfully to determine the amino acids. The derivatization reaction conditions as well as the variables of the headspace sampling were optimized. The existence of a matrix effect was checked and the analytical characteristics of the method were determined. The limits of detection were $0.15-2.89 \mathrm{mg} / \mathrm{L}$, and the limits of quantification were $0.46-8.67 \mathrm{mg} / \mathrm{L}$. The instrumental repeatability was $1.6-11.5 \%$. The quantification of the amino acids in six urine samples from healthy subjects was performed with the method developed with the one-point standard additions protocol, with norleucine as the internal standard.

Keywords: Amino acids / Derivatization / Gas chromatography-mass spectrometry / Headspace / Programmed temperature vaporizer

DOI 10.1002/jssc.201600186

Additional supporting information may be found in the online version of this article at the publisher's web-site

\section{Introduction}

The metabolic profile in biofluids represents a snapshot of ongoing biological processes in the human body. Metabolomic profiling, or metabolomics, heralds the promise of earlier disease detection and improved understanding of systems biology [1]. In this context, owing to their vital role in many metabolomic pathways, amino acids are one of the most important targets for metabolomic profiling studies. Several metabolic disorders and diseases can be diagnosed by determining amino acid concentration levels [2-6]. Although some amino acids have also been studied and proposed as candidate cancer biomarkers [7-17], their validity as cancer biomarkers remains unclear, and more studies and analysis of a large number of samples are needed [18]. However, the development of new analytical methods is a very important

Correspondence: Dr. Carmelo García Pinto, Departamento de Oúmica Analíca Nutricióny Bromatologia. Químicas, Universidad de Salamanca, 37008 Salamanca, Spain

E-mail: cgp@ usal.es

Abbreviations: ECF, ethyl chloroformate; HS, headspace; PTV, programmed temperature vaporizer; SIM, selected ion monitoring; UHO, ultra-high quality point for the research and discovery of sensitive biomarkers and for early disease detection $[18,19]$.

Several analytical techniques have been reported for the determination of amino acids. CE has been extensively used for their analysis in different matrices, with different detection systems, such as indirect UV [20,21], conductivity [22], LIF $[23,24]$ and MS $[11,25-28]$. LC, HPLC, and UHPLC have also been applied for the determination of amino acids $[9,17,29-35]$. GC has been used to analyze amino acids both with a flame ionization detector $[36,37]$ and with MS. This las coupling has been reported to be a powerful analytical tool with many advantages for the simultaneous identification and quantification of multiple compounds in different biological samples, especially when it is applied to investigate human body fluids [38]. The determination of amino acids with GCMS has been applied in several biological matrices [2,39-42], including blood [43] and urine samples [2, 17, 42, 44-46].

Owing to the presence of highly polar groups $\left(-\mathrm{NH}_{2}\right.$, $-\mathrm{COOH})$ and their nonvolatile nature, amino acids need to be derivatized for GC-MS analysis [2]. Most derivatization reactions use apolar reagents, which require organic solvents that are not directly compatible with aqueous media. By contrast, chloroformates have been widely used as derivatizing agents of amino acids, with the advantages of in situ derivatization in aqueous medium at room temperature $[36,37,39-41,44,47,48]$. The derivatization of amino 
acids with isobutyl chloroformate forms a large number of byproducts [2] and may result in some nonvolatile derivatized compounds. Ethyl chloroformate has been used satisfactorily for the derivatization of amino acids in urine samples $[2,42,45,46]$. Nevertheless, in these cases, the derivatives are extracted from the mixture before injection into GC by LLE, liquid-liquid microextraction or SPME. The coupling of derivatization with headspace sampling, as proposed in this paper, removes this extraction step and makes the use of any additional pretreatment to isolate the derivatives from the matrix unnecessary, therefore reducing errors associated with sample treatment and solvent consumption. Moreover, the use of headspace avoids interferences from the nonvolatile compounds of the matrix. Taking this all into account, the coupling of derivatization and headspace sampling is therefore a good alternative for the analysis of derivatized amino acids in different samples.

The aim of the present work was to develop a new method for the determination of amino acids. The strategy can be divided into two main parts. The first one is a very simple manual step in which the sample and all of the reagents are added to the vial so that the derivatization reaction can occur. The second is a completely automated procedure in which the sample vial is placed in the sampler and the volatile derivatized amino acids are separated and analyzed by headspace sampling coupled to a programmed temperature vaporizer, followed by GC-MS determination. To the best of our knowledge, this is the first time that derivatization coupled to headspace sampling has been proposed for the determination of these analytes.

\section{Materials and methods}

\subsection{Chemicals and standard solutions}

The analytes employed (L-alanine, sarcosine, $\mathrm{N}$-ethylglycine, DL-valine, L-leucine, and DL-proline), the internal standard (norleucine), ethyl chloroformate (ECF), and pyridine were supplied by Sigma-Aldrich (Steinheim, Germany). Ethanol, sodium chloride, sodium hydroxide, and sodium dihydrogen phosphate dihydrate were purchased from Scharlau (Barcelona, Spain). The ultra-high quality water (UHQ) used was obtained with a Wasserlab Ultramatic water purification system (Noain, Spain).

A stock standard solution of each analyte $(1000 \mathrm{mg} / \mathrm{L})$ was prepared in UHQ water. The solutions were kept refrigerated at $4^{\circ} \mathrm{C}$ and diluted to the desired concentration when used. These solutions were used to spike the water and urine samples at the different concentrations analyzed.

\subsection{Apparatus}

Headspace (HS) sampling was performed with a MPS2 MultiPurpose Sampler (Gerstel, Mühlheim an der Ruhr, Germany). This sampler is equipped with a tray for
32 consecutive samples and an oven with positions for six sample vials. A $2.5 \mathrm{~mL}$ syringe at $120^{\circ} \mathrm{C}$ was used.

Analyses of the compounds were performed on a GC-MS instrument (Agilent Technologies, Santa Clara, CA, USA) consisting of an Agilent 7890A series gas chromatograph equipped with a 6890 Agilent Technologies programmed temperature vaporizer (PTV) injector and an Agilent 5975 Cinert XL MSD. A glass wool liner $(71 \mathrm{~mm} \times 2 \mathrm{~mm})$ was used in the PTV injector. Cooling was accomplished with liquid $\mathrm{CO}_{2}$. Analytes were separated on a DB-VRX column $(20 \mathrm{~m} \times$ $0.18 \mathrm{~mm} \times 1.00 \mu \mathrm{m}$ ) (J\&W Scientific, Folsom, CA, USA). The carrier gas was helium N50 (99.005\% pure; Air Liquide) at a flow rate of $1.8 \mathrm{~mL} / \mathrm{min}$. The detector was a quadrupole mass spectrometer. Data acquisition was performed with MSD ChemStation, v. E.02.00.493 software from Agilent Technologies. Identification of the compounds was confirmed using the NIST 98 database (NIST/EPA/NIH Mass Spectral Library, version 2.0).

\subsection{Derivatization reaction}

$\mathrm{NaCl}(1.5 \mathrm{~g})$ was weighed in a $10 \mathrm{~mL}$ headspace vial with a screw cap and $3 \mathrm{~mL}$ of sample was added. Forty microliters of $1 \mathrm{mM} \mathrm{NaOH}, 120 \mu \mathrm{L}$ of ethanol and $100 \mu \mathrm{L}$ of pyridine were added to provide a suitable medium for the derivatization reaction. Following this, $120 \mu \mathrm{L}$ of ECF was added as the derivatization reagent and the mixture (with the vial closed) was shaken for $30 \mathrm{~s}$ at $1600 \mathrm{rpm}$ with a Vortex device. The vial was then opened to remove the carbon dioxide generated during the reaction. Finally, the vial was again closed and placed in the headspace sampler.

Urine samples were centrifuged at $5000 \mathrm{rpm}$ for $10 \mathrm{~min}$ before adding the reagents.

\subsection{Headspace programmed-temperature vaporizer} gas chromatography with MS

To generate the headspace, samples were shaken at $750 \mathrm{rpm}$ for $30 \mathrm{~min}$ at $90^{\circ} \mathrm{C}$. Then $2.4 \mathrm{~mL}$ was injected in solventvent injection mode by a PTV system. The conditions used were as follows: an initial temperature of $70^{\circ} \mathrm{C}$ for $0.80 \mathrm{~min}$ a vent flow of $20 \mathrm{~mL} / \mathrm{min}$, a vent pressure of $5.00 \mathrm{psi}$, and venting time of $0.75 \mathrm{~min}$. Once venting had finished, the split valve was closed and the injection system was flash-heated a $720^{\circ} \mathrm{C} \mathrm{min}{ }^{-1}$ up to $250^{\circ} \mathrm{C}$. The analytes were then transferred from the liner to the capillary column $(1.0 \mathrm{~min})$. Finally, the split valve was opened and the liner temperature was held at $250^{\circ} \mathrm{C}$ until the end of the chromatographic analysis.

The column oven temperature program used an initial temperature of $50^{\circ} \mathrm{C}$ for $2 \mathrm{~min}$; an increase at $60^{\circ} \mathrm{C} \mathrm{min}-1$ up to $175^{\circ} \mathrm{C}$ and then an increase at $45^{\circ} \mathrm{C} \mathrm{min}^{-1}$ up to $240^{\circ} \mathrm{C}$, finally holding for $1 \mathrm{~min}$. The total chromatographic run time was $6.53 \mathrm{~min}$.

The ion source, the quadrupole, and the transfer line temperature were 230,150 , and $275^{\circ} \mathrm{C}$, respectively. A solvent 
delay time of $3.50 \mathrm{~min}$ and $70 \mathrm{eV}$ for the electron-ionization mode were established. The detector was set at scan acquisition mode during the variable optimization step and once the optimal conditions for the analysis had been selected, it was set at the synchronous selected ion monitoring (SIM)/scan mode. Although with this mode it is possible to lose some sensitivity, it allows to obtain more information about the sample, since it provides the collection of both SIM (selected ion monitoring) and full-scan data in a single run. The full scan mode $\left(40-550 \mathrm{~m} / z, 9.76\right.$ scans s$\left.^{-1}\right)$ was used for identification and the SIM mode for quantification with a dwell time of $1 \mathrm{~ms}$. The quantifier (in bold) and qualifier ions selected for derivatized compounds were 116, 44, 189 for alanine and sarcosine; 130, 58, 203 for ethylglycine; 144, 116, 217 for valine; 158, 102, 231 for leucine and norleucine (internal standard) and 142, 70, 215 for proline.

\section{Results and discussion}

3.1 Optimization of the derivatization reaction conditions

As reported in the Introduction, the derivatization of amino acids with ethyl chloroformate has been used previously for their analysis with GC $[2,42,45,46]$. The variables studied to determine the optimum conditions for derivatization were the $\mathrm{pH}$ value and the volume of pyridine, ethanol, and ethyl chloroformate. These studies were performed with UHQ water samples at a concentration of $10 \mathrm{mg} / \mathrm{L}$ for all the analytes.

Values of $\mathrm{pH}$ in the range of $6-12$ were tested. In all cases, $40 \mu \mathrm{L}$ of the appropriate buffer solution was added to $3 \mathrm{~mL}$ of sample, together with $120 \mu \mathrm{L}$ of ethanol, $100 \mu \mathrm{L}$ of pyridine, and $120 \mu \mathrm{L}$ of ECF. A pH value of 9 provided the highest signal for most of the compounds together with suitable reproducibility values.

The volume of pyridine was optimized using a constant volume of $120 \mu \mathrm{L}$ of ethanol, $120 \mu \mathrm{L}$ ECF, and at $\mathrm{pH} 9$. Volumes of $60,80,100,120$, and $140 \mu \mathrm{L}$ were assayed. The signal area increased up until a volume of pyridine of $100 \mu \mathrm{L}$. For larger volumes, the areas decreased, as shown in Fig. 1A. Based on this result, $100 \mu \mathrm{L}$ of pyridine was chosen.

The volumes of ethanol and ECF were optimized simultaneously to minimize the number of byproducts formed. Thus, equal volumes of ethanol and ECF were added to each of the volumes tested. Values of $80,100,120,140$, and $160 \mu \mathrm{L}$ were assayed at a constant volume of $100 \mu \mathrm{L}$ of pyridine, at $\mathrm{pH}$ 9. The highest derivatization efficiency was obtained when $120 \mu \mathrm{L}$ of ethanol and $120 \mu \mathrm{L}$ of ECF were added, as can be observed in Fig. 1B.

\subsection{Headspace optimization}

To determine the optimum conditions for headspace generation, equilibration temperature, equilibration time, and sample volume variables were studied by means of a multivariate experimental design due to the interactions between them. Temperature affects both equilibrium and equilibration time. Moreover, the time required for equilibrium to be reached may also be dependent upon sample volume. To study the effect of each of these three variables individually, and their interactions on the analytical signal, a Box-Behnken experimental design was used. This design allows the generation of response surfaces by using three levels of each facto studied. In all cases, UHQ water samples spiked with analyte concentrations of $10 \mathrm{mg} / \mathrm{L}$ were used. Each of the experiments was performed in triplicate to detect any lack of fit of the data in the model generated. The response chosen was the peak area corresponding to the base peak of the spectrum of each of the derivatized compounds. Only the model that included the variables, their squares and the interactions did not show a lack of fit.

The values evaluated were temperature (A), 70, 80, and $90^{\circ} \mathrm{C}$; time (B), 10, 20, and $30 \mathrm{~min}$, and sample volume (C) $1,2.5$, and $4 \mathrm{~mL}$. As may be seen in Supporting Information Table S1, the significant variables were temperature (A) for all the compounds; volume (C) for alanine, sarcosine, ethyl glycine, and valine; the interaction between temperature and sample volume $(\mathrm{AC})$ for proline, and the squares of temperature (AA) for alanine, sarcosine, ethylglycine, and valine, and of the sample volume (CC) for proline.

The equilibration temperature value that provided the maximum signal for all compounds was $90^{\circ} \mathrm{C}$. Although the results obtained regarding time and sample volume were less conclusive, short times and low sample volumes provided sig. nals with low reproducibility for most cases (between 17 an $52 \%$ ), the reason why a time of $30 \mathrm{~min}$ and a sample volume of $3 \mathrm{~mL}$ were chosen. Despite that with these values the maximum signals were not reached, proper signals were obtained for all compounds together with suitable reproducibility values $(2-12 \%)$ for the entire process including derivatization and instrumental determination.

\subsection{Programmed temperature vaporizer gas} chromatography with MS optimization

The variables studied in the PTV-GC-MS system were venting temperature, venting flow, and venting time for the PTV injector and dwell time for the MS detector. All these variables were studied with UHQ water samples spiked at a concentration of $10 \mathrm{mg} / \mathrm{L}$ for all the analytes.

The solvent-vent injection mode was selected for the PTV injector and a glass wool liner was chosen. The initial temperature of the liner in which solvent purging occurs, or venting temperature, was studied in the range of $20-90^{\circ} \mathrm{C}$. It was observed that the signal areas increased gradually with increasing the initial temperature of $20^{\circ} \mathrm{C}$ up to $70^{\circ} \mathrm{C}$. For values higher than $70^{\circ} \mathrm{C}$ the signal decreased, displaying a greater decrease for the more volatile analytes. Accordingly, a venting temperature of $70^{\circ} \mathrm{C}$ was chosen. Regarding the purge gas flow, values of $10,20,35$, and $50 \mathrm{~mL} / \mathrm{min}$ were studied. 
A
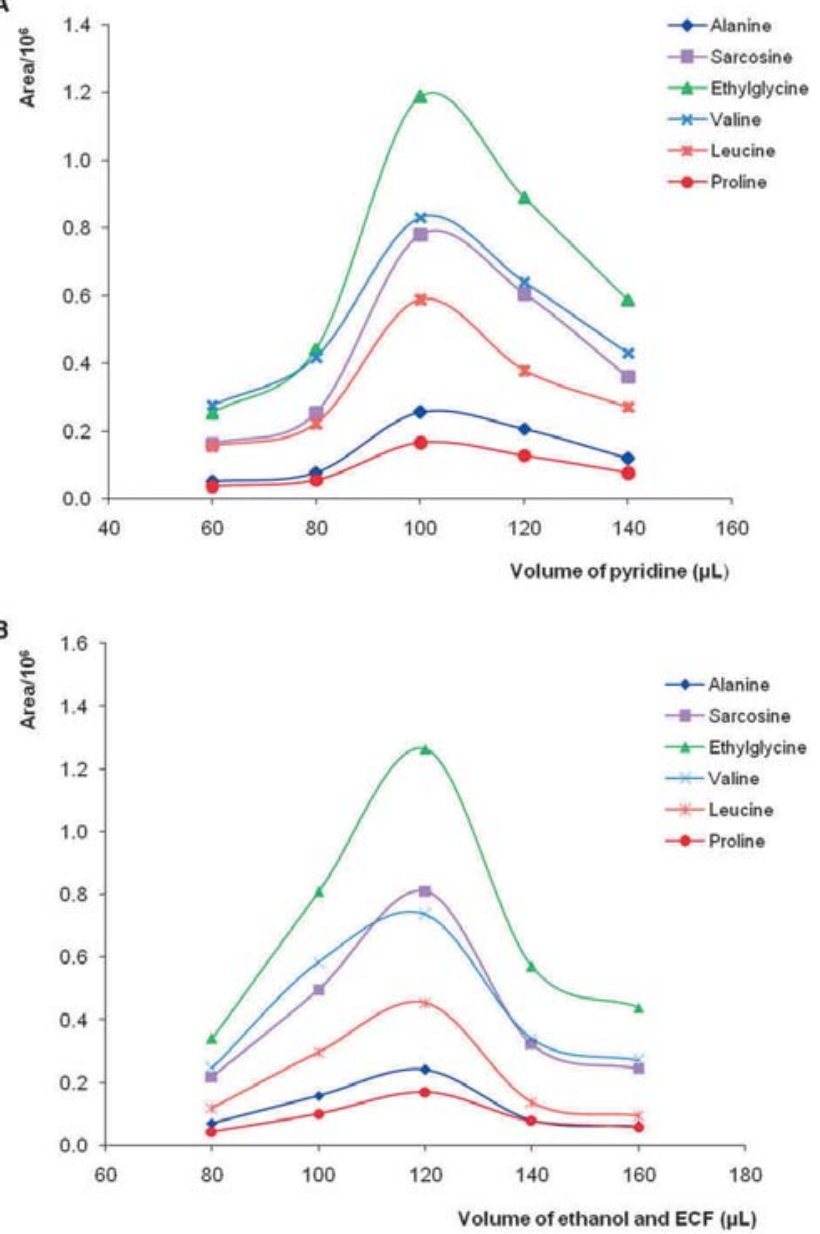

Figure 1. Optimization of (A) volume of pyridine and (B) volume of ethanol and

Similar signals were obtained for all values and hence $20 \mathrm{~mL} / \mathrm{min}$ was chosen as this was the value with better reproducibility and also saved in the use of the carrier gas. Different venting times $(0.5,0.75$, and $1 \mathrm{~min})$ were assayed. Problems in reproducibility were observed for venting times of 0.5 and $1 \mathrm{~min}$, probably because the solvent was not completely removed for 0.5 min and because a fraction of the analytes was eliminated with the solvent for $1 \mathrm{~min}$. Maximum signals for all compounds and the best reproducibility were obtained for $0.75 \mathrm{~min}$ and was therefore selected as the appropriate venting time.
Dwell times of 1, 10, 50, and $100 \mathrm{~ms}$ in the SIM mode were assayed. One millisecond afforded the best peak definition with no significant increased noise. Accordingly, $1 \mathrm{~ms}$ was used as the dwell time. Figure 3 shows the extracted ion chromatogram of a urine sample spiked with all the analytes under the optimized conditions.

\subsection{Analytical characteristics of the method}

For amino acid detection, it is not possible to use an analytefree urine matrix for the preparation of the calibration curves. 


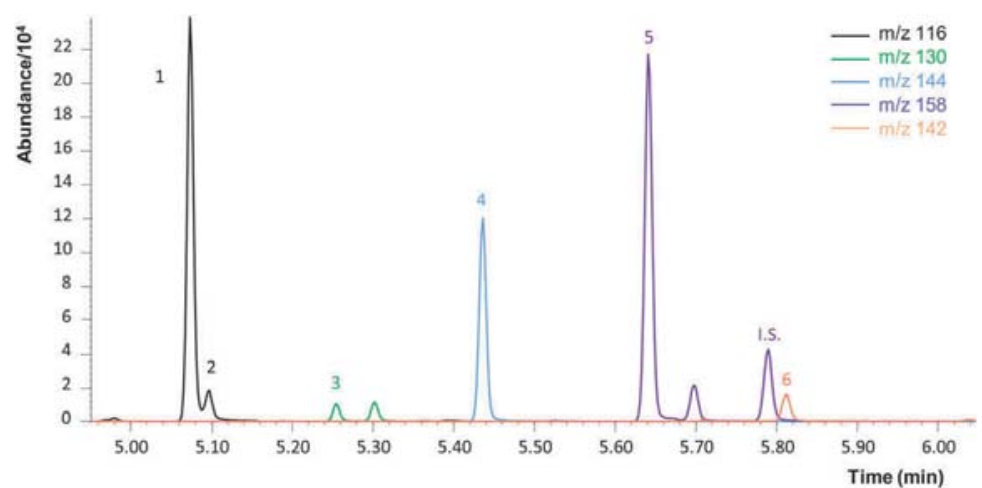

Figure 2. Chromatogram provided by a urine sample spiked with all the analytes at the concentrations of the high level shown in Table 1 and subjected to the optimized derivatization and analytical procedure. 1 . alanine; 2 . sarcosine; 3 . ethylglycine; 4 . valine; 5 . leucine; IS. norleucine; 6 . proline.

Table 1. Analytical characteristics of the method

\begin{tabular}{|c|c|c|c|c|c|c|c|}
\hline & \multirow[t]{2}{*}{$R^{2}$} & \multicolumn{2}{|c|}{$\begin{array}{l}\text { Concentration } \\
\text { level (mg/L) }\end{array}$} & \multicolumn{2}{|c|}{$\begin{array}{l}\text { Instrumental } \\
\text { repeatability (RSD \%) }\end{array}$} & \multirow[t]{2}{*}{ LOD (mg/L) } & \multirow[t]{2}{*}{ LOQ (mg/L) } \\
\hline & & Low level & High level & Low level & High level & & \\
\hline Alanine & 0.9918 & 5.0 & 40 & 4.3 & 5.8 & 1.20 & 3.60 \\
\hline Sarcosine & 0.9838 & 0.375 & 3.0 & 11.5 & 5.9 & 0.21 & 0.87 \\
\hline Ethylglycine & 0.9913 & 0.25 & 2.0 & 10.2 & 4.6 & 0.15 & 0.46 \\
\hline Valine & 0.9819 & 1.25 & 10 & 10.3 & 3.4 & 0.97 & 2.90 \\
\hline Leucine & 0.9952 & 1.25 & 10 & 3.5 & 1.6 & 0.37 & 1.10 \\
\hline Proline & 0.9844 & 1.25 & 10 & 6.1 & 4.4 & 2.89 & 8.67 \\
\hline
\end{tabular}

Standard curves were obtained by analyzing aqueous standard solutions of the analytes at five concentration levels ranging from the low level to the high level shown at Table 1 for each compound, using norleucine as an internal standard to compensate for variations of the whole process. Each concentration level was subjected to the derivatization procedure and analyzed four times. All the calibrations exhibited linear behavior, with good values for the coefficient of determination $\left(R^{2}\right)$, as shown in Table 1 . The validity of the models generated was checked using analysis of variance, and none of the models generated was found to show a lack of fit. Instrumental repeatability was determined at two concentration levels where each derivatized standard solution was injected four times. The values (expressed as RSD) are shown in Table 1 and ranged respectively between 3.5 and $11.5 \%$ for the low level and between 1.6 and $5.9 \%$ for the high level. Table 1 shows the LODs and LOQs calculated, respectively, as 3.3 and 10 times the SD of a water blank divided by the slopes of the calibration curves. The LODs of the proposed method ranged from 0.15 to $2.89 \mathrm{mg} / \mathrm{L}$, whereas the LOQs ranged from 0.46 to $8.67 \mathrm{mg} / \mathrm{L}$.

\subsection{Urine samples}

To check for possible matrix effects and to investigate the applicability of the method to real urine analyses, a comparison between the signals obtained with aqueous and urine samples, from three different subjects, was made. Samples were spiked at a concentration of $5 \mathrm{mg} / \mathrm{L}$ for all the analytes. Significant differences were observed between the urine samples and UHQ samples. To avoid this, the possibility of sample dilution with UHQ water was evaluated. Different dilution ratios up to 1:6 were assayed. Figure 3 shows the comparison between the signals obtained with aqueous samples and urine samples from three subjects at a dilution ratio of 1:6. As can be seen in the figure, the dilution ratio of 1:6 did not eliminate the matrix effect either. To overcome this matrix effect, a standard additions protocol was proposed for the determination of these analytes in urine samples; as this technique had already been shown to work appropriately for urine samples [17].

Owing to the existence of a matrix effect, and to simplify the protocol for the determination of amino acids in urine 


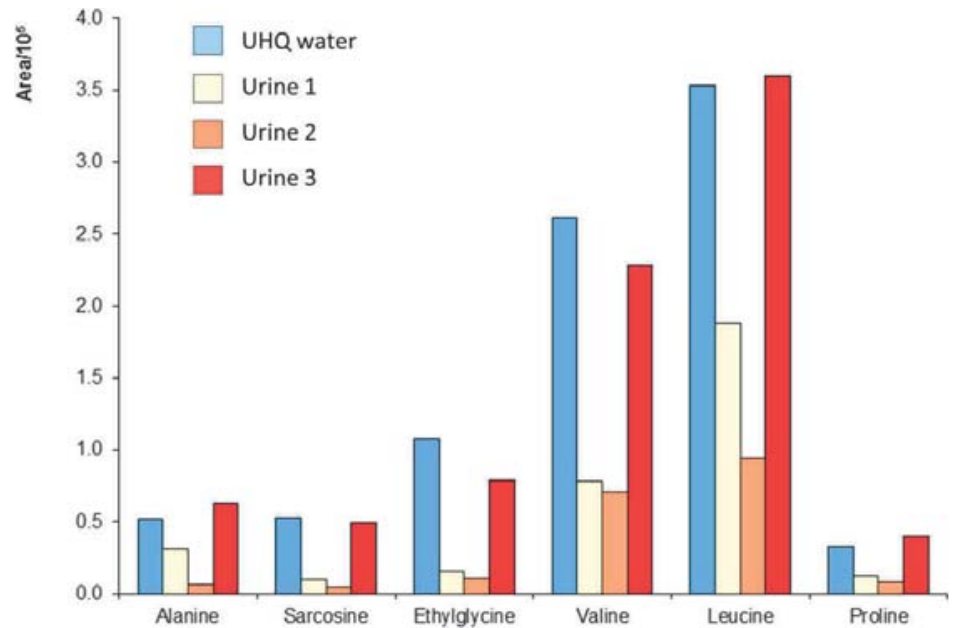

Figure 3. Comparison between the signals obtained with UHO water and with urine samples from three subjects at a dilution ratio of 1:6.

Table 2. Results obtained for the compounds studied in urine samples

\begin{tabular}{|c|c|c|c|c|c|c|}
\hline Sample & Alanine & Sarcosine & Ethylglycine & Valine & Leucine & Proline \\
\hline Urine 1 & $(4 \pm 1) 10$ & $0.5 \pm 0.1$ & $<\mathrm{LOD}$ & $4 \pm 1$ & $2.5 \pm 0.3$ & $0.9 \pm 0.1$ \\
\hline Urine 2 & $(5 \pm 1) 10$ & $0.5 \pm 0.2$ & $<\mathrm{LOD}$ & $5.3 \pm 0.9$ & $4.5 \pm 0.5$ & $1.4 \pm 0.3$ \\
\hline Urine 3 & $(5 \pm 1) 10$ & $0.4 \pm 0.1$ & $<\mathrm{LOD}$ & $5 \pm 2$ & $3.0 \pm 0.5$ & $0.6 \pm 0.2$ \\
\hline Urine 4 & $26 \pm 4$ & $0.4 \pm 0.1$ & $<\mathrm{LOD}$ & $4.3 \pm 0.6$ & $3.0 \pm 0.2$ & $1.1 \pm 0.1$ \\
\hline Urine 5 & $32 \pm 6$ & $0.27 \pm 0.09$ & $<$ LOD & $3.8 \pm 0.6$ & $3.1 \pm 0.5$ & $0.8 \pm 0.2$ \\
\hline Urine 6 & $14 \pm 9$ & $<\mathrm{LOD}$ & $<\mathrm{LOD}$ & $1.2 \pm 0.5$ & $1.2 \pm 0.2$ & $0.59 \pm 0.09$ \\
\hline
\end{tabular}

Concentrations are given as $\mu \mathrm{mol}$ analyte/mmol creatinine.

samples, a comparison between a conventional standard additions (six-level calibration study) and a one-point standard additions was carried out. The quantification of these compounds in a urine sample from a healthy subject with both protocols was performed. No significant differences in the concentrations calculated were found between the conventional and the one-point standard additions. Accordingly, the one-point standard additions protocol was proposed for the determination of these analytes in urine samples. This strategy makes the final procedure simpler and faster.

The developed method was applied to the analysis of six urine samples from healthy subjects to quantify the amino acids and estimate the concentration of these endogenous compounds in urine samples. A one-point standard additions protocol with norleucine as the internal standard was proposed for the accurate determination of the analytes. A modification of the Jaffè method based on the photometric detection of a reddish complex resulting from the reaction between creatinine and picric acid was used to normalize the analyte concentrations to the total creatinine concentration $[49,50]$. The concentrations are expressed normalized to the creatinine concentration: $\mu \mathrm{mol}$ analyte $/ \mathrm{mmol}$ creatinine. Table 2 shows the results obtained. The improvement with the internal standard is higher for leucine and proline, compounds that have a chromatographic behavior similar to norleucine.

\section{Concluding remarks}

A new coupling of derivatization of amino acids to HS-PTVGC-MS has been developed. The derivatization of analytes with ethyl chloroformate in aqueous medium is a great advantage when biological samples such as urine are analyzed. The coupling of derivatization with headspace sampling proposed in this work allows for the elimination of solvents or others treatments to extract the derivatives from the matrix before their injection into GC. This instrumental configuration minimizes sample manipulation and therefore, reduces 
solvent consumption and the errors associated with this step of the analysis.

The analytical characteristics of the method were evaluated, which included the observation of linear behavior, the absence of lack of fit, suitable reproducibility, and good limits of detection. The calibration strategy used was the one-point standard additions protocol with an internal standard, the results obtained being highly satisfactory.

Based on the favorable results obtained in this work, the application of the proposed methodology in future research studies can hopefully be used to analyze a large number of urine samples from both healthy subjects and cancer patients to evaluate the possible differences in the level of these analytes between both groups.

Financial support from DGI (CTQ2013-47993-P) and from the Consejería de Educación y Cultura of the Junta de Castilla $y$ León (SA162U14) is acknowledged. R.M.G.P. is grateful for a predoctoral grant from the University of Salamanca. The authors wish to thank the Languages Service of the University of Salamanca for supervising the English version of this manuscript.

The authors declare to have no conflict of interests.

\section{References}

[1] Shanaiah, N., Desilva, M. A., Nagana Godwa, G. A., Raftery, M. A., Hainline, B. E., Raftery, D., Class selection of amino acid metabolites in body fluids using chemical derivatization and their enhanced 13C NMR. Proc. Natl. Acad. Sci. USA 2007, 104, 11540-11544.

[2] Mudiam, M. K. R., Ratnasekhar, C., Jain, R., Saxena, P. N., Chauhan, A., Murthy, R. C., Rapid and simultaneous determination of twenty amino acids in complex biological and food samples by solid phase microextraction and gas chromatography-mass spectrometry with the aid of
experimental design after ethyl chloroformate derivatization. J. Chromatogr. B 2012, 907, 56-64.

[3] Chace, D. H., Sherwin, J. E., Hillman, S. I., Lorey, F., Cunningham, G. C., Use of phenylalanine-to-tyrosine ratio determined by tandem mass spectrometry to improve newborn screening for phenylketonuria of early discharge specimens collected in the first 24 hours. Clin. Chem. 1998, 44, 2405-2409.

[4] Chace, D. H., Hillman, S. L., Millington, S. L., Millington, D. S., Kahler, S. G., Roe, C. R., Naylor, E. W., Rapid diagnosis of maple syrup urine disease in blood spots from newborns by tandem mass spectrometry. Clin. Chem. 1995, 41, 62-68.

[5] Yang, R., Wang, S., Sun, L., Liu, J., Li, H., Sui, X., Wang, M., Xiu, H., Wang, S., He, Q., Dong, J., Chen, W., Association of branched-chain amino acids with coronary artery disease: a matched-pair case-control
Cardiovasc. Dis. 2015, 25, 937-942.

[6] Shatla, H. M., Tomoum, H. Y., Elsayed, S. M., Elagouza, I. A., Shatla, R. H., Mohsen, M. M., Hamed, A. N., Role of plasma amino acids and urinary organic acids in diagnosis of mitochondrial diseases in children. Pediatr. Neurol. 2014, 51, 820-825.
[7] Armitage, E. G., Barbas, C., Metabolomics in cancer biomarker discovery: current trends and future perspectives. J. Pharm. Biomed. Anal. 2014, 87, 1-11.

[8] Mamtimin, B., Kedena, K. B., Hasim, A., Matsidik, A. Hizbulla, M., Kurbantay, N., Upur, H., Plasma amino acid profiling of cancer patients with abnormal Savda based on high-performance liquid chromatography. J. Tradit. Chin. Med. 2014, 34, 560-565.

[9] Cheng, F., Wang, Z., Huang, Y., Duan, Y., Wang, X., Investigation of salivary free amino acid profile for early diagnosis of breast cancer with ultra-performance liquid chromatography- mass spectrometry. Clin. Chim. Acta
2015, 447, 23-31.

[10] Trock, B. J., Application of metabolomics to prostate cancer. Urol. Oncol. Semin. Ori. 2011, 29, 572-581.

[11] Soliman, L. C., Hui, Y., Hewavitharana, A. K., Chen, D. D. Y., Monitoring potential prostate cancer biomarkers in urine by capillary electrophoresis-tandem mass spectrometry. J. Chromatogr. A 2012, 1267, 162-169.

[12] Bellance, N., Pabst, L., Allen, G., Rossignol, R., Nagrath, D., Oncosecretomics coupled to bioenergetics identifies $\alpha$-amino adipic acid, isoleucine and GABA as potentia biomarkers of cancer: differential expression of c-Myc Oct1 and KLF4 coordinates metabolic changes. Biochim. Biophys. Acta 2012, 1817, 2060-2071.

[13] Sreekumar, A., Poisson, L. M., Rajendiran, T. M., Khan A. P., Cao, Q., Yu, J., Laxman, B., Mehra, R., Lonigro, R. J., Li, Y., Nyati, M. K., Ahsan, A., Kalyana-Sundaram, S., Han, B., Cao, X., Byun, J., Omenn, G. S., Ghosh, D., Pennathur, S., Alexander, D. C., Berger, A., Shuster, J. R., Wei, J. T., Varambally, S., Beecher, C., Chinnaiyan, A. M., Metabolomic profiles delineate potential role for sarcosine in prostate cancer progression. Nature 2009 457, 910-914.

[14] Wu, H., Liu, T., Ma, C., Xue, R., Deng, C., Zeng, H., Shen X., GC/MS-based metabolomic approach to validate the role of urinary sarcosine and target biomarkers for human prostate cancer by microwave-assisted derivatization. Anal. Bioanal. Chem. 2011, 401, 635-646.

[15] Pasikanti, K. K., Esuvaranathan, K., Ho, P. C., Mahendran, R., Kamaraj, R., Wu, Q. H., Chiong, E., Chan, E. C. Y., Noninvasive urinary metabonomic diagnosis of human bladder cancer. J. Proteome Res. 2010, 9, 2988-2995.

[16] Alberice, J. V., Amaral, A. F. S., Armitage, E. G. Lorente, J. A., Algaba, F., Carrilho, E., Márquez, M. García, A., Malats, N., Barbas, C., Searching for urine biomarkers of bladder cancer recurrence using a liquid chromatography-mass spectrometry and capillary electrophroresis-mass spectrometry metand capillary proach. J. Chromatogr. A 2013, 1318, 163-170.

[17] Shamsipur, M., Naseri, M. T., Babri, M., Quantification of candidate prostate cancer metabolite biomarkers in urine using dispersive derivatization liquidliquid microextraction followed by gas and liquid chromatography-mass spectrometry. J. Pharm. Biomed. Anal. 2013, 81-82, 65-75.

[18] Issaq, H. J., Waybright, T. J., Veenstra, T. D., Cancer biomarker discovery: opportunities and pitfalls in analytical methods. Electrophoresis 2011, 32, 967-975.

[19] Kouremenos, K. A., Johansson, M., Marriott, P. J., Advances in gas chromatographic methods for the 
identification of biomarkers in cancer. J. Cancer 2012, $3,404-420$.

[20] Xu, X., Jia, Z., Shu, Y., Liu, L., Dynamic pH junctionsweeping technique for on-line concentration of acidic amino acids in human serum by capillary electrophoresis with indirect UV detection. J. Chromatogr. B 2015, 980, 20-27.

[21] Soga, T., Imaizumi, M., Capillary electrophoresis method for the analysis of inorganic anions, organic acids, amino acids, nucleotides, carbohydrates and other anionic compounds. Electrophoresis 2001, 22, 3418-3425.

[22] Kubáň, P., Boček, P., On-line coupling of a clean-up device with supported liquid membrane to capillary electrophoresis for direct injection and analysis of serum and plasma samples. J. Chromatogr. A 2012, 1234, 2-8.

[23] Turkia, H., Sirén, H., Penttilä, M., Pitkänen, J.-P., Capillary electrophoresis with laser-induced fluorescence detecelectrophoresis with laser-induced fluorescence detec-
tion for studying amino acid uptake by yeast during beer tion for studying amino acid uptake by yea.

[24] Lorenzo, M. P., Navarrete, A., Balderas, C., García, A., Optimization and validation of a CE-LIF method for amino acid determination in biological samples. J. Pharm. Biomed. Anal. 2013, 73, 116-124.

[25] Schiavone, N. M., Sarver, S. A., Sun, L., Wojcik, R., Dovichi, N. J., High speed capillary zone electrophoresismass spectrometry via an electrokinetically pumped mass spectrometry via an electrokinetically pumped and a protein digest. J. Chromatogr. B 2015, 991, 53-58.

[26] Soga, T., Heiger, D., Amino acid analysis by capillary electrophoresis electrospray ionization mass spectrometry. Anal. Chem. 2000, 72, 1236-1241.

[27] Wakayama, M., Aoki, N., Sasaki, H., Ohsugi, R., Simultaneous analysis of amino acids and carboxylic acids by capillary electrophoresis-mass spectrometry using an acidic electrolyte and uncoated fused-silica capillary. Anal. Chem. 2010, 82, 9967-9976.

[28] Atherton, T., Croxton, R., Baron, M., González-Rodríguez, J., Gámiz-Gracia, L, García-Campaña, A. M., Analysis of amino acids in latent fingerprint residue by capillary electrophoresis-mass spectrometry. J.Sep. Sci. 2012,35, 2994-2999.

[29] Held, P. K., White, L., Pasquali, M., Quantitative urine amino acid analysis using liquid chromatography tandem mass spectrometry and aTRAQ ${ }^{\oplus}$ reagents. J. Chromatogr. B 2011, 879, 2695-2703.

[30] Song, Y., Funatsu, T., Tsunoda, M., Amino acids analysis using a monolithic silica column after derivatiza-
tion with 4-fluoro-7-nitro-2,1,3-benzoxadiazole (NBD-F). J. Chromatogr. B 2011, 879, 335-340.

[31] Gogami, T., Okada, K., Oikawa, T., High-performance liquid chromatography analysis of naturally occurring $D$ amino acids in sake. J. Chromatogr. B 2011, 879, 32593267.

[32] Yoshida, H., Kondo, K., Yamamoto, H., Kageyama, N., Ozawa, S., Shimbo, K., Muramatsu, T., Imaizumi, A., Mizukoshi, T., Masuda, J., Nakayama, D., Hayakawa, Y., Watanabe, K.. Mukaibatake, K., Miyano, H., Validation of an analytical method for human plasma free amino acids by high-performance liquid chromatography ionization mass - erformance liquid chromatography ionderivatization. J. Chromatogr. B 2015, 998, 88-96.
33] Tuberoso, C. I. G., Cagiu, F., Serreli, G., Mameli, S., Determination of dansylated amino acids and biogenic amines in Cannonau and Vermentiono wines by HPLC FLD. Food Chem. 2015, 175, 29-35.

[34] Moldoveanu, S. C., Zhu, J., Qian, N., Free amino acids analysis by liquid chomatography with tandem mas spectrometry in several botanicals with antioxidant character. J. Sep. Sci. 2015, 38, 2208-2222.

[35] Zhang, J., Zhao, C., Chang, Y., Zhao, Y., Li, Q., Lu, X., Xu, G. Analysis of free amino acids in flue-cured tobacco G., Analysis of $\mathrm{C}, \mathrm{Cha}$ a leaves using ultra-high peformance liquid chromatography with single quadrupole mass spectrometry. J. Sep.
Sci. 2013, 36, 2868-2877.

36] Hušek, P., Rapid derivatization and gas chromatographic determination of amino acids. J. Chromtogr. 1991, 552, 289-299.

[37] Matsumura, S., Kataoka, H., Makita, M., Determination of amino acids in human serum by capillary gas chroof amino acids in human serum by capillary gas

[38] Kałużna-Czaplińska, J., Jóźwik, J., Current applications of chromatographic methods for diagnosis and identification of potential biomarkers in cancer. Trends Anal. Chem. 2014, 56, 1-12.

[39] Villas-Bôas, S. G. Delicado, D. G., Akesson, M., Nielsen, J., Simultaneous analysis of amino and nonamino organic acids as methyl chloroformate derivatives using gas chromatography-mass spectrometry. Anal. using gas chromatography-mas
Biochem. 2003, 322, 134-138.

[40] Zampolli, M. G., Basaglia, G., Dondi, F., Sternberg, R. Szopa, C., Pietrogrande, M. C., Gas chromatographymass spectrometry analysis of amino acid enantiomers as methyl chloroformate derivatives: application to space analysis. J. Chromatogr. A 2007, 1150, 162-172.

[41] Chen, W. P., Yang, X. Y., Hegeman, A. D., Gray, W. M., Cohen, J. D., Microscale analysis of amino acids using gas chromatography-mass spectrometry after methyl chloroformate derivatization. J. Chromatogr. B 2010, 878, 2199-2208.

[42] Mudiam, M. K. R., Ratnasekhar, C., Ultra sound assisted one step rapid derivatization and dispersive liquid-liquid me step rapic de. Raver microextraction followed by gas chromatography-mass spectrometric determination of amino acids inc

[43] Kawana, S., Nakagawa, K., Hasegawa, Y., Yamaguchi, S., Simple and rapid analytical method for detection of amino acids in blood using blood spot of filter paper, fast-GC/MS and isotope dilution technique. J. Chromatogr. B 2010, 878, 3113-3118.

[44] Kvitvang, H. F. N., Andressean, T., Adam, T., Villas-Bôas, S. G., Bruheim, P., Highly sensitive GC/MS/MS method for quantification of amino and nonamino organic acids. Anal. Chem. 2011, 83, 2705-2711.

[45] Qiu, Y., Su, M., Liu, Y., Chen, M., Gu, J., Zhang, J., Jia, W., Application of ethyl chloroformate derivatization for gas chromatography-mass spectrometry based metabonomic profiling. Anal. Chim. Acta 2007, 583, 277283.

[46] Namera, A., Yashiki, M., Nishida, M., Kojima, T., Direct extract derivatization for determination of amino acids in human urine by gas chromatography and mass spectrometry. J. Chromatogr. B 2002, 776, 49-55. 
[47] Hušek, P., Chloroformates in gas chromatography as general purpose derivatizing agents. J. Chromatogr. B $1998,717,57-91$.

[48] Huang, Z.-H., Wang, J., Gage, D. A., Watson, J. T., Sweely, C. C., Characterization of $\mathrm{N}$-ethoxycarbonyl ethyl esters of amino acids by mass spectrometry. J. Chromtogr. $1993,635,271-281$
[49] Jaffè Ueber den Niederschlag, M., welchen Pikrinsäure im normalen Harn-erzeugt und über eine neue Reaction des Kreatinins. Z. Physiol. Chem. 1886, 10, 391-

[50] Delanghe, J. R., Speeckaert, M. M., Creatinine determination according to Jaffè-what does it stand for? NDT Plus 2011, 4, 83-86. 


\title{
DERIVATIZATION COUPLED TO HEADSPACE PROGRAMMED- TEMPERATURE VAPORIZER GAS CHROMATOGRAPHY WITH MASS SPECTROMETRY FOR THE DETERMINATION OF AMINO ACIDS:
}

\section{APPLICATION TO URINE SAMPLES}

\author{
Rosa María González Paredes, Carmelo García Pinto*, José Luis Pérez Pavón and \\ Bernardo Moreno Cordero
}

Departamento de Química Analítica, Nutrición y Bromatología. Facultad de Ciencias Químicas, Universidad de Salamanca. 37008 Salamanca, SPAIN

*Corresponding author: (fax) +34-923-294483; (e-mail) cgp@usal.es

\author{
SUPPORTING INFORMATION
}


1 Table 1

2 Results of the experimental design ( $p$ values). A, temperature; B, equilibration time; C,

3 volume.

\begin{tabular}{llllllllll}
\hline Compound & A & B & C & AB & AC & BC & AA & BB & CC \\
\hline Alanine & 0.0000 & 0.4963 & 0.0000 & 0.9222 & 0.2524 & 0.1273 & 0.0294 & 0.1129 & 0.1386 \\
Sarcosine & 0.0000 & 0.3620 & 0.0000 & 0.9203 & 0.5726 & 0.0940 & 0.0110 & 0.0942 & 0.2229 \\
Ethylglycine & 0.0000 & 0.4736 & 0.0000 & 0.9744 & 0.7568 & 0.1190 & 0.0044 & 0.1065 & 0.1617 \\
Valine & 0.0000 & 0.9767 & 0.0000 & 0.8978 & 0.6624 & 0.3867 & 0.0046 & 0.2112 & 0.4935 \\
Leucine & 0.0000 & 0.2426 & 0.2922 & 0.4573 & 0.1123 & 0.4586 & 0.5421 & 0.3219 & 0.4035 \\
Proline & 0.0000 & 0.6331 & 0.3970 & 0.8941 & 0.0036 & 0.5190 & 0.0698 & 0.1526 & 0.0048 \\
\hline
\end{tabular}

4 



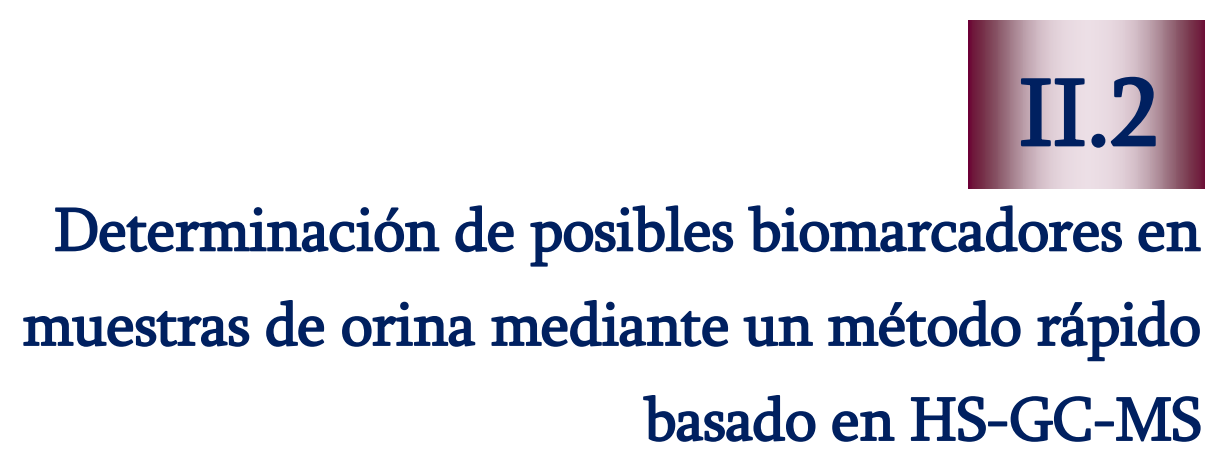



Artículo de investigación II.2

Resumen 

La orina tiene una utilidad considerable como fluido biológico para diagnóstico de enfermedades; de hecho, el análisis de orina se usa rutinariamente para un gran número de pruebas. En los últimos años se ha incrementado significativamente el uso de orina para los estudios de metabolómica debido al interés en la búsqueda de nuevos biomarcadores que se puedan medir en muestras obtenidas de forma no invasiva. Además, la orina, comparada con otros fluidos biológicos para su uso en metabolómica, tiene como ventajas el gran número de metabolitos que contiene, su bajo contenido de proteínas, la facilidad de manipulación y preparación de muestra y el hecho de que el volumen no es un factor limitante.

El potencial de diagnóstico de compuestos orgánicos volátiles (volatile organic compounds, VOCs) para diferentes enfermedades ha sido explorado durante mucho tiempo y ha generado un interés creciente. El estudio de biomarcadores volátiles en muestras clínicas ofrece una alternativa para el desarrollo de herramientas rápidas y baratas de detección de enfermedades. Buena parte de los estudios de biomarcadores volátiles se han llevado a cabo en muestras de aliento, aunque también se han investigado otras matrices como orina, heces, sudor y sangre.

Entre los numerosos compuestos volátiles descritos en bibliografía como posibles biomarcadores, para este trabajo se seleccionaron el disulfuro de dimetilo (DMDS), tolueno, 1,4-xileno, 2-octanona y 2,6-dimetil-7-octen2-ol. Esta selección incluye compuestos con diferentes grupos funcionales y volatilidades, de forma que podrían considerarse compuestos representativos de la familia química a la que pertenecen. Aunque algunos de ellos han sido propuestos como posibles biomarcadores de cáncer de próstata, todavía se 
necesitan más estudios para su confirmación y se requieren, por tanto, nuevas metodologías capaces de determinarlos de forma rápida y sensible.

Las innegables ventajas del muestreo mediante espacio de cabeza, como la facilidad de preparación de muestra, de implementación y automatización, hacen que sea una buena opción para la determinación de compuestos volátiles en un amplio rango de concentraciones y en diferentes tipos de muestras. Casi todos los métodos propuestos para la determinación de compuestos volátiles de la orina que utilizan el muestreo mediante espacio de cabeza llevan a cabo una etapa adicional de preconcentración mediante, por ejemplo, microextracción en fase sólida. Eliminar esta etapa de preconcentración, tal y como se propone en este trabajo, hace el procedimiento final más simple y rápido.

El desarrollo de nuevos métodos rápidos, sencillos y sensibles para la detección y cuantificación de ciertos compuestos en fluidos biológicos humanos es esencial, no sólo para la investigación y descubrimiento de nuevos biomarcadores, sino también para la confirmación de la utilidad de aquellos ya descritos. Para este último propósito, la propuesta de metodologías rápidas es también el primer paso necesario para analizar un gran número de muestras tanto de individuos sanos como de pacientes para establecer las diferencias en el nivel de estos analitos entre ambos grupos y poder llegar así a una detección temprana de la enfermedad.

En este sentido, el objetivo de este trabajo era desarrollar un método rápido y sencillo basado en HS-GC-MS para la determinación en orina de cinco compuestos descritos como posibles biomarcadores. Con la información de que disponemos, éste es el primer trabajo basado en el uso de 
HS-GC-MS para la determinación simultánea de estos cinco compuestos en orina. La propuesta de un método capaz de determinar conjuntamente todos estos compuestos de diferentes familias químicas y descritos como posibles biomarcadores puede ser muy útil para estudios posteriores de confirmación.

Para llevar a cabo el objetivo planteado es necesario optimizar las variables involucradas en el método. Para ello, el primer paso fue determinar las condiciones óptimas para la generación del espacio de cabeza, evaluándose variables como el $\mathrm{pH}$, el volumen de muestra y el tiempo de equilibrado.

En primer lugar, se realizó un estudio comparativo entre $\mathrm{pH}$ ácido y fisiológico con una orina dopada con los cinco compuestos estudiados. Para tener el mismo volumen de espacio de cabeza y poder realizar así la comparación entre $\mathrm{pH}$ ácido y fisiológico, se añadieron $240 \mu \mathrm{L}$ de ácido fosfórico para obtener $\mathrm{pH}$ ácido y $240 \mu \mathrm{L}$ de metanol en su lugar para el $\mathrm{pH}$ fisiológico. Excepto para el DMDS, no se observó un gran aumento de las señales con $\mathrm{pH}$ ácido, incluso para el 2,6-dimetil-7-octen-2-ol la señal disminuía. Teniendo en cuenta estos resultados y con el objetivo de reducir la variabilidad y evitar un mayor consumo de tiempo y reactivos, se seleccionó pH fisiológico sin la adición de ningún reactivo.

Con respecto al volumen de muestra se probaron valores de 1 a $5 \mathrm{~mL}$, observándose que las áreas de las señales aumentaban con el aumento del volumen de muestra y seleccionándose, por tanto, $5 \mathrm{~mL}$. Al utilizar viales de espacio de cabeza de $10 \mathrm{~mL}$, no se probaron volúmenes mayores de $5 \mathrm{~mL}$ para evitar salpicaduras en el septum y contaminación de la aguja de la jeringa de espacio de cabeza. 
Con un volumen de $5 \mathrm{~mL}$, se optimizó el tiempo de equilibrado probándose valores de 5 a $30 \mathrm{~min}$. Sólo se observaron ligeras diferencias de señal entre los diferentes tiempos, si bien los valores mejores de repetibilidad se obtuvieron para $15 \mathrm{~min}$ y, por tanto, este tiempo fue el seleccionado.

En relación al cromatógrafo de gases, se probaron diferentes programas de temperatura del horno con el fin de conseguir la mejor separación de los analitos en el menor tiempo posible, estableciéndose finalmente, las rampas máximas permitidas por el equipo.

En cuanto al sistema de detección se optimizó la velocidad de barrido y el tiempo de permanencia en el espectrómetro de masas. En el modo scan, el valor establecido como velocidad de barrido se utiliza para calcular el número de veces que se registra la abundancia de cada masa antes de pasar a la siguiente masa. Con un rango de masas de 35 a 250 se evaluaron valores de velocidad de barrido de $0,1,2$ y 3, lo que daba lugar a 18.11, 11.06, 6.22 y 3.11 scan s$^{-1}$, respectivamente. La mejor morfología de pico para todos los compuestos se obtenía con un valor de 1 (11.06 scan s $\left.\mathrm{s}^{-1}\right)$. También se optimizó el tiempo de permanencia en el modo SIM, probándose valores de entre 1 y 100 ms. Con el objeto de obtener la mejor definición de pico sin un aumento significativo del ruido, se seleccionó un valor de tiempo de permanencia para cada compuesto: $35 \mathrm{~ms}$ para el disulfuro de dimetilo, $10 \mathrm{~ms}$ para el tolueno, el 1,4-xileno y 2-octanona y $1 \mathrm{~ms}$ para el 2,6-dimetil-7octen-2-ol.

El efecto de matriz se evaluó mediante la comparación de las señales obtenidas con una disolución acuosa y dos muestras de orinas, dopadas todas con los analitos objeto de estudio. Se observaron diferencias entre las 
muestras de orina y la disolución acuosa, por lo que se probaron diferentes diluciones de la orina con agua. Aunque para dos de los compuestos parecía eliminarse el efecto de matriz con una dilución 1:5, para el resto no se conseguía eliminar, lo que ponía de manifiesto la necesidad de llevar a cabo la calibración mediante adición estándar. Con el fin de simplificar el procedimiento de cuantificación de estos compuestos en orina, se propuso la adición estándar de un punto. Esta estrategia ya había sido evaluada y comparada con la adición estándar convencional en el trabajo previo de determinación de aminoácidos en orina (II.1), mostrando buenos resultados y haciendo el proceso final más simple y rápido.

Las características analíticas del método se determinaron con una orina dopada a diez niveles de concentración. Las rectas de calibración obtenidas mostraban comportamiento lineal, sin fallo de ajuste y con valores de coeficiente de determinación superiores a 0.99 para todos los compuestos. La repetibilidad y reproducibilidad se determinaron a dos niveles de concentración (1 y $10 \mu \mathrm{g} \mathrm{L}^{-1}$ ), siendo los valores obtenidos menores del 9.5\% y del 14.2\%, respectivamente. Los límites de detección (LOD) se encontraban entre 0.01 y $0.48 \mu \mathrm{g} \mathrm{L}^{-1}$, mientras que los límites de cuantificación (LOQ) oscilaban entre 0.05 y $1.43 \mu \mathrm{g} \mathrm{L}{ }^{-1}$.

Para validar el método optimizado, se doparon cinco orinas de individuos sanos con todos los compuestos a una concentración conocida y se analizaron con el método desarrollado, aplicando la adición estándar de un punto mencionada anteriormente. Las concentraciones calculadas para las muestras de orina eran significativamente iguales a las concentraciones reales 
a las que se habían dopado las muestras, de forma que el intervalo de confianza de la predicción incluía la concentración real.

Además, se llevó a cabo la determinación de estos analitos en tres muestras de orina con el método desarrollado y se realizó la normalización a la concentración total de creatinina en cada una de las muestras. En las muestras analizadas se encontraron para algunos compuestos concentraciones próximas al límite de detección. Los resultados obtenidos ponen de manifiesto la validez de la metodología propuesta para determinar concentraciones muy bajas de estos posibles biomarcadores.

Como conclusión principal, podemos destacar que se ha desarrollado un método simple y rápido basado en HS-GC-MS para la determinación en orina de algunos posibles biomarcadores. Esta metodología combina las ventajas de utilizar muestras de orina obtenidas de forma no invasiva con las del muestreo mediante espacio de cabeza, lo que la convierte en una estrategia adecuada para la determinación de estos posibles biomarcadores volátiles. Además, la determinación de compuestos con diferentes grupos funcionales que se lleva a cabo demuestra que el método propuesto podría aplicarse para determinar conjuntamente otros compuestos pertenecientes a las familias químicas estudiadas en este trabajo.

El método analítico propuesto en este trabajo se puede utilizar para estudios posteriores, permitiendo analizar de forma rápida y sencilla un gran número de muestras tanto de individuos sanos como de pacientes para establecer así las diferencias en los niveles de concentración de estos compuestos entre ambos grupos y permitir, por tanto, evaluar su utilidad como biomarcadores. 
Artículo de investigación II.2

Enviado para publicación 

Journal of Separation Science

Journal of Separation Science

$$
\mathrm{VCH}
$$

Determination of possible biomarkers in urine samples by a rapid method based on headspace-gas chromatographymass spectrometry

\begin{tabular}{|r|l|}
\hline Journal: & Journal of Separation Science \\
\hline Manuscript ID & Draft \\
\hline Watey - Manuscript type: & Original Paper \\
\hline Complete List of Authors: & $\begin{array}{l}\text { González Paredes, Rosa María; University of Salamanca, Analytical } \\
\text { Chemistry, Nutrition and Bromatology } \\
\text { García Pinto, Carmelo; University of Salamanca, Analytical Chemistry, } \\
\text { Nutrition and Bromatology } \\
\text { Pavon, Jose Luis Perez; University of Salamanca, Analytical Chemistry } \\
\text { Moreno Cordero, Bernardo; University of Salamanca, Analytical Chemistry }\end{array}$ \\
\hline Keywords: & $\begin{array}{l}\text { Biomarkers, Gas chromatography-mass spectrometry, Headspace, Urine } \\
\text { samples }\end{array}$ \\
\hline &
\end{tabular}


Determination of possible biomarkers in urine samples by a rapid method based on headspace-gas chromatography-mass spectrometry

Bernardo Moreno Cordero Químicas, Universidad de Salamanca. 37008 Salamanca, SPAIN

*Corresponding author: (fax)+34-923-294483; (e-mail) cgp@usal.es

12 Non-standard abbreviations

ANOVA analysis of variance

HS headspace

PTV programmed temperature vaporizer

SIM selected ion monitoring

UHQ ultra-high quality samples 


\section{Abstract}

The development of new analytical strategies plays a vital role for the research, discovery and confirmation of analytes that can be sensitive biomarkers. Within this line of work, a method based on headspace-gas chromatography-mass spectrometry has been developed and validated for the determination of possible biomarkers (dimethyl disulfide, toluene, 1,4-xylene, 2-octanone and 2,6-dimethyl-7-octen-2-ol) in human urine samples.

The existence of a matrix effect was checked and the analytical characteristics of the method were determined. The limits of detection ranged from 0.01 to $0.48 \mu \mathrm{g} \mathrm{L}^{-1}$, whereas the limits of quantification ranged from 0.05 to $1.43 \mu \mathrm{g} \mathrm{L}^{-1}$. The repeatability was below $9.5 \%$ and reproducibility below $14.2 \%$. Five urine samples from healthy subjects spiked with the possible biomarkers were used to validate the proposed methodology. The quantification of these compounds in three urine samples from healthy subjects was performed with the one-point standard additions protocol. 


\section{$47 \quad 1$ Introduction}

Urine has considerable value as a diagnostic biofluid [1] and for centuries, physicians have attempted to use urine for the non-invasive assessment of disease [2], in fact, urinalysis is routinely used for a great number of tests [3]. The use of urine as a sample for metabolomics studies has increased significantly in last years, due to the interest in searching for new biomarkers that can be measured in samples obtained by non-invasive means [2]. Besides being a biofluid obtained non-invasively, urine has many others advantages for use as sample for metabolomics compared to other human biofluids, such as its large number of metabolites and low protein content, the ease of handling and sample preparation, and that the sample volume is not a limiting factor [4]. for different diseases has been explored for a long time and has generated increasing interest [5-8]. The study of volatile biomarkers in clinical samples offers an alternative for developing rapid and potentially inexpensive disease screening tools [5]. Most of the studies on volatile biomarkers have been carried out on exhaled-breath samples [8-18], although other matrices, such as urine $[8,19-24]$ and feces $[25,26]$, have also been investigated.

Among the numerous volatile compounds described in literature as possible biomarkers, dimethyl disulfide, toluene, 1,4-xylene, 2-octanone and 2,6-dimethyl-7octen-2-ol have been selected for this work. This selection includes inorganic and organic compounds with different functional groups and volatility, so they could be considered representative compounds of the chemical family which they belong to. Although some of them have been proposed as possible biomarkers of prostate cancer [27, 28], further studies are still needed for their confirmation, which first requires the development of

new methodologies for their determination. 
The undeniable advantages of headspace sampling, such as easy sample preparation, environmental-friendliness and easiness of implementation and automation makes it the preparation technique of choice for the analysis of volatile compounds in a wide range of concentrations and sample types [29, 30]. Nearly all proposed methods for the determination of urinary volatile compounds using headspace sampling perform an additional preconcentration step, such as solid phase microextraction [2, 19, 24, 31-33]. Avoiding this preconcentration step, as proposed herein, minimizes sample handling and, hence, makes the final procedure simpler and faster.

The development of new rapid, simple and sensitive analytical methods for the detection and quantification of certain compounds in human biofluids is essential, not only for the research and discovery of new biomarkers but also for the confirmation of the usefulness of the ones previously described. For this last purpose, the proposal of rapid methodologies is also the first necessary step to analyze a large number of samples from both healthy and patient individuals to establish the differences in the levels of these analytes between both groups for a future early disease detection.

In this sense, the aim of the present work was to develop a rapid and simple method based on headspace (HS)-gas chromatography (GC)-mass spectrometry (MS) for the determination of some possible biomarkers in human urine. Five volatile possible biomarkers (dimethyl disulfide, toluene, 1,4-xylene, 2-octanone and 2,6-dimethyl-7octen-2-ol) with different functional groups and volatility have been selected. The proposal of an analytical method able to determine jointly all these compounds from different chemical families and which have been described as possible biomarkers can be urine. 
2 Materials and Methods

\subsection{Chemicals and standard solutions}

Dimethyl disulfide (DMDS), 2-octanone and 2,6-dimethyl-7-octen-2-ol 1,4-xylene (1,4-dimethylbenzene) were supplied by Acros Organics (Geel, Belgium). Methanol HPLC grade was provided by Sigma-Aldrich (Steinheim, Germany). Sodium chloride and phosphoric acid reagent grade were purchased from Scharlau (Barcelona, Spain). The ultra-high quality water (UHQ) used was obtained with a Wasserlab Ultramatic water purification system (Noain, Spain). Stock solutions ( 2500 or $1000 \mathrm{mg} \mathrm{L}^{-1}$ ) of each analyte were prepared in methanol. The solutions were kept refrigerated at $4{ }^{\circ} \mathrm{C}$ and diluted to the desired concentration to prepare the working solutions containing the five compounds in methanol. These working solutions were used to spike the water and urine samples at the different concentrations analyzed.

\subsection{Apparatus}

The instrumental configuration HS-GC-MS as well as the data acquisition software were equivalent to that described previously [34].

\subsection{Sample pretreatment}

Urine samples obtained from apparently healthy volunteers were stored at $-20{ }^{\circ} \mathrm{C}$. For the analysis, the samples were left at room temperature until they had thawed completely. Then, they were transferred to a $15-\mathrm{mL}$ glass centrifuge tube with screw cap (Scharlau, Spain) and were centrifuged for $10 \mathrm{~min}$ at $5000 \mathrm{rpm}$. To analyze the spiked samples, $2.5 \mathrm{~g}$ of $\mathrm{NaCl}$ was weighed in a $10-\mathrm{mL}$ headspace vial and $5 \mathrm{~mL}$ of urine and 50 $\mu \mathrm{L}$ of the working solution with the five compounds were added. 


\subsection{HS-GC-MS}

To generate the headspace, samples were shaken at $750 \mathrm{rpm}$ for $15 \mathrm{~min}$ at $90{ }^{\circ} \mathrm{C}$. Then $2.4 \mathrm{~mL}$ was injected using the hot split mode $(1: 3)$ at $250{ }^{\circ} \mathrm{C}$ by a programmed temperature vaporizer (PTV) system equipped with a liner packed with Tenax-TA ${ }^{\mathbb{B}}$. Oven temperatures were as follows: initially, $50{ }^{\circ} \mathrm{C}$ for $2 \mathrm{~min}$, followed by heating at 120 ${ }^{\circ} \mathrm{C} \min ^{-1}$ to $70{ }^{\circ} \mathrm{C}$, then increased at $95{ }^{\circ} \mathrm{C} \mathrm{min}^{-1}$ to $115^{\circ} \mathrm{C}$, heated at $65^{\circ} \mathrm{C} \mathrm{min}^{-1}$ to 175 ${ }^{\circ} \mathrm{C}$, and finally heated at $45^{\circ} \mathrm{C} \min ^{-1}$ to $240{ }^{\circ} \mathrm{C}$, holding this temperature for $2 \mathrm{~min}$. The total chromatographic run time was $7.00 \mathrm{~min}$.

The ion source, the quadrupole, and the transfer line temperatures were $230{ }^{\circ} \mathrm{C}$, $150{ }^{\circ} \mathrm{C}$, and $275^{\circ} \mathrm{C}$, respectively. A solvent delay time of $1.50 \mathrm{~min}$ and $70 \mathrm{eV}$ for the electron-ionization mode were established. The detector was set at scan acquisition mode during the variable optimization step and once the optimal conditions for the analysis had been selected, it was set at the synchronous SIM/scan mode. This mode allows to obtain more information about the sample, since it provides the collection of both SIM (selected ion monitoring) and full-scan data in a single run. The full scan mode $(35-250 \mathrm{~m} / \mathrm{z}, 11.06$ scans $\mathrm{s}^{-1}$ ) was used for identification and the SIM mode for quantification with a dwell time of $35 \mathrm{~ms}$ for dimethyl disulfide, $10 \mathrm{~ms}$ for toluene, 1,4-xylene and 2-octanone and 1 ms for 2,6-dimethyl-7-octen-2-ol. The quantifier (in bold) and qualifier ions selected for analytes were 94, 79, 45 for dimethyl disulfide; 91, 92, 65 for toluene; 91, 106, 105 for 1,4-xylene; 43, 58, 41 for 2-octanone; and 59, 41, 43 for 2,6-dimethyl-7-octen-2-ol.

\section{Results and discussion}

3.1 Experimental procedure 
To determine the optimum conditions for headspace generation, the variables studied were: $\mathrm{pH}$, sample volume and equilibration time. All these variables were studied with urine samples spiked at a concentration of $10 \mu \mathrm{g} \mathrm{L}^{-1}$ for all the analytes.

A comparative study between acidic and physiological $\mathrm{pH}$ was performed. To obtain acidic $\mathrm{pH}$, a volume of $240 \mu \mathrm{L}$ of phosphoric acid was added to the vial, whereas for the physiological $\mathrm{pH} 240 \mu \mathrm{L}$ of methanol was added in its place to have the same headspace volume in both cases and thus enabling the comparison. No large increase in signal was observed (Fig. 1) by adding acid, except for dimethyl disulfide. Moreover, the signal for 2,6-dimethyl-7-octen-2-ol decreased with acidic $\mathrm{pH}$. According to this and with the purpose of reducing the variability and avoiding a greater expenditure of time and consumption of reagents, physiological $\mathrm{pH}$ without the addition of any reagent was selected.

Regarding sample volume, values of $1,2,3,4$ and $5 \mathrm{~mL}$ of urine were assayed at physiological $\mathrm{pH}$ and $20 \mathrm{~min}$ of equilibration time. The proportion of salt/sample volume was maintained for each sample volume. As it can be observed in Fig. 2a, the signal areas increased gradually when increasing the sample volume, therefore a volume of $5 \mathrm{~mL}$ was selected. Volumes greater than $5 \mathrm{~mL}$ were not tested when using 10 -mL headspace vials to prevent splashing of the septum and contamination of the needle of the headspace sampler.

Equilibration times of 5, 10, 15, 20, 25 and 30 min were assayed at a constant volume of $5 \mathrm{~mL}$ of urine sample and at physiological $\mathrm{pH}$. Only slight differences were observed (Fig. 2b) between the different times; however, the lowest values of RSD were obtained for $15 \mathrm{~min}$. This time was selected as optimum for later studies.

Regarding the GC-MS system, oven temperatures of the gas chromatograph and sampling rate and dwell times in the mass spectrometry detector were optimized. Several 
temperature programs of the oven were assayed with the aim of achieving the best separation of the analytes in the shortest possible time. Finally, the ramps set were the maximum ones permitted by the chromatograph configuration.

In the scan mode, the value set as the sampling rate is used to calculate the number of times the abundance of each mass is recorded before going on to the next mass, and therefore it must be optimized. With a mass range from 35 to 250 , values of 0 , 1,2 and 3 of sampling rate were assayed, resulting in 18.11, 11.06, 6.22 and 3.31 scan s$^{-}$ ${ }^{1}$, respectively. The best morphology for all the compounds was obtained with a sampling rate of $1\left(11.06\right.$ scan s$\left.^{-1}\right)$. Dwell times of $1,10,35,50,75$ and $100 \mathrm{~ms}$ in the SIM mode were assayed. In order to obtain the best peak definition with no significant increased noise, a value of dwell time for each compound was selected: $35 \mathrm{~ms}$ for dimethyl disulfide, $10 \mathrm{~ms}$ for toluene, 1,4-xylene and 2-octanone and $1 \mathrm{~ms}$ for 2,6-dimethyl-7octen-2-ol.

\subsection{Matrix effect}

To evaluate the possible existence of a matrix effect, a comparison between the signals obtained with aqueous and urine samples, from two different subjects, was made. Samples were spiked at a concentration of $10 \mu \mathrm{g} \mathrm{L}^{-1}$ for all the analytes. Significant differences were observed between the urine samples and UHQ samples. Dilution of urine samples up to a ratio of 1:5 (1 mL urine $+4 \mathrm{~mL}$ UHQ) did not remove the matrix effect (Fig. 3). Although for 2-octanone and 2,6-dimethyl-7-octen-2-ol the matrix effect seemed to be overcome with a dilution ratio of 1:5, for dimethyl disulfide, toluene and 1,4-xylene the matrix effect was not eliminated, and therefore a standard additions protocol was required. In order to simplify the protocol for the determination of these compounds in urine samples, a one-point standard additions protocol was proposed. This 
strategy has been previously tested and used [34], showing suitable results and making the final procedure simpler and faster.

\subsection{Analytical characteristics of the method}

Standard curves were obtained to determine the analytical characteristics of the method by analyzing a urine sample spiked at ten concentration levels in the calibration ranges shown in Table 1. Each concentration level was analyzed in triplicate. The analytical characteristics of the methods are shown in Table 1. The calibration models displayed linear behavior. The validity of the models generated was checked using ANOVA, and none of the models generated was found to show a lack of fit. The value of the correlation determination coefficient $\left(\mathrm{R}^{2}\right)$ was higher than 0.99 for all the compounds.

Repeatability and reproducibility were determined using a urine spiked with the compounds at two concentration levels $\left(1\right.$ and $\left.10 \mu \mathrm{g} \mathrm{L}^{-1}\right)$. Each concentration level was analyzed five times on the same day for repeatability and on two different days (five replicates per day) for reproducibility. The values (expressed as relative standard deviation, RSD) are shown in Table 1, being below $9.5 \%$ for repeatability and $14.2 \%$ for reproducibility. The limits of detection (LODs) and the limits of quantification (LOQs) calculated, respectively, as 3.3 and 10 times the standard deviation of the urine blank divided by the slopes of the calibration curves, are also shown in Table 1 . The LODs of the proposed method ranged from 0.01 to $0.48 \mu \mathrm{g} \mathrm{L}^{-1}$, whereas the LOQs ranged from 0.05 to $1.43 \mu \mathrm{g} \mathrm{L}^{-1}$.

\subsection{Method validation}

To validate the optimized method, five urine samples from healthy subjects were spiked with all the compounds at a known concentration and analyzed with the developed method. The one-point standard additions protocol was used for the accurate 
determination of the analytes. The urine samples were analyzed before being spiked, and the area of the signals obtained for each compound was subtracted to the signals of the standard additions. Table 2 shows the calculated concentration and the actual concentration for all possible biomarkers in the spiked urines. The calculated concentrations for the urine samples were close to the actual concentrations at which the samples were spiked, such that the confidence interval of prediction included the actual concentration.

The developed method was applied to the analysis of three urine samples from healthy subjects to quantify the possible biomarkers. The one-point standard additions protocol proposed was applied. Normalization of the analyte concentrations to the total creatinine concentration is common when urine samples are used. For this purpose, a modification of the Jaffè method was used [35,36]. Table 3 shows the concentrations obtained both expressed in $\mu \mathrm{g} / \mathrm{L}$ as normalized to the creatinine concentration: $\mathrm{nmol}$ analyte/mmol creatinine. Fig. 4 shows the extracted ion chromatograms of a spiked UHQ water sample, a non-spiked urine sample and a spiked urine sample. The concentrations selected to spike the UHQ water and the urine were those found for each compound in the non-spiked urine sample. These chromatograms together with the concentrations determined in the urine samples shown in Table 3, confirm the validity of the proposed method to determine very low concentrations, in some cases close to the limit of quantification.

\section{Concluding remarks}

A rapid and simple method for the determination of some possible biomarkers in urine based on HS-GC-MS has been developed. This methodology combines the advantages of using urine samples obtained non-invasively with those of the headspace sampling, making it a suitable approach for the determination of these volatile possible 
Page 11 of 24

\author{
Journal of Separation Science
}

biomarkers. In addition, the determination performed herein of compounds with different functional groups, demonstrates that the proposed method could be applied to jointly determine other compounds belonging to the chemical families studied in this work.

The method developed allowed quantification in urine samples of five compounds described in literature as possible biomarkers by means of one-point standard additions method. The analytical characteristics of the method showed linear behavior, no lack of fit, suitable repeatability and reproducibility values (below $9.5 \%$ and $14.2 \%$, respectively) and low limits of detection and quantification (ranging from 0.01 to $0.48 \mu \mathrm{g}$ $\mathrm{L}^{-1}$ and from 0.05 to $1.43 \mu \mathrm{g} \mathrm{L}^{-1}$, respectively). The validation of the proposed method was carried out determining the concentration of the compounds in five spiked urine samples and the results obtained were highly satisfactory.

The analytical method proposed herein can hopefully be used for further studies, allowing to analyze quickly and easily a large number of samples from both healthy and patient individuals, in order to establish the differences in the concentration level of these compounds between both groups, and allowing therefore, to assess their usefulness as biomarkers

\title{
Acknowledgments
}

Financial support from DGI (CTQ2013-47993-P) and from the Consejería de Educación y Cultura of the Junta de Castilla y León (SA162U14) is acknowledged. R.M.G.P. is grateful for a predoctoral grant from the University of Salamanca. The authors wish to thank the Languages Service of the University of Salamanca for supervising the English version of this manuscript.

\section{Conflict of interest}

The authors declare to have no conflict of interests. 
References

[1] Bouatra S., Aziat F., Mandal R., Chi Guo A., Wilson M. R., Knox C., Bjorndahl T. C., Krishnamurthy R., Saleem F., Liu P., Dame Z. T., Poelzer J., Huynh J., Yallou F. S., Psychogios N., Dong E., Bogumil R., Roehring C., Wishart D. S., The human urine metabolome. PLoS ONE 2013, 8(9):e73076. doi:10.1371/journal.pone.0073076.

[2] Rigau M., Olivan M., García M., Sequeiros T., Montes M., Colás E., Llauradó M., Planas J., Torres I., Morote J., Cooper C., Reventós J., Clark J., Doll A., The present and future of prostate cancer urine biomarkers. Int. J. Mol. Sci. 2013, 14, 12620-12649.

[3] Smith S., Burden H., Persad R., Whittington K., Costello B. L., Ratcliffe N. M., Probert C. S., A comparative study of the analysis of human urine headspace using gas chromatography-mass spectrometry. J. Breath. Res. 2008, 2, 037022 (10pp). doi:10.1088/1752-7155/2/3/037022.

[4] Lima A. R., Bastos M. L., Carvalho M., Pinho P. G., Biomaker discovery in human prostate cancer: an update in metabolomics studies. Transl. Oncol. 2016, 9, 357370

[5] Sethi S., Nanda R., Chakraborty T., Clinical application of volatile organic compound analysis for detecting infectious diseases. Clin. Microbiol. Rev. 2013, 26, $462-475$.

[6] Monteiro M. S., Carvalho M., Bastos M. L., Pinho P. G., Potentiality of volatile organic compounds to discriminate patients with cancer by using chemometric tools, in: S. Rudaz (Ed.), Identification and Data Processing methods in metabolomics. 2015, pp. 166-184. 
[7] Schmidt K., Podmore I., Current challenges in volatile organic compounds analysis as potential biomarkers of cancer. $J$. Biomarkers 2015, 2015, 1-16.

[8] Shirasu M., Tauhara K., The scent of disease: volatile organic compounds of the human body related to disease and disorder. J. Biochem. 2011, 150, 257-266.

[9] Miekisch W., Schubert J. K., Gabriele F. E., Noelge-Schomburg G. F. E., Diagnostic potential of breath analysis-focus on volatile organic compounds. Clin. Chim. Acta 2004, 347, 25-39

[10] Amann A., Smith D. (ed), Breath analysis for clinical diagnosis and therapeutic monitoring, World Scientific, Singapore, Republic of Singapore 2005.

[11] Cao W., Duan Y., Breath analysis: potential for clinical diagnosis and exposure assessment. Clin. Chem. 2006, 52, 800-811

[12] Smith D., Spanel P., The challenge of breath analysis for clinical diagnosis and therapeutic monitoring. Analyst 2007, 132, 390-396.

[13] Boots A. W., van Berker J. J. B. N, Dallinga J. W., Smolinska A., Wouters M. F. M., van Schooten F. J., The versatile use of exhaled volatile organic compounds in human health and disease. J. Breath Res. 2012, 6, 027108 (21pp) doi:10.1088/1752$7155 / 6 / 2 / 027108$.

[14] Dummer J., Storer M., Swanney M., McEwan M., Scott-Thomas A., Bhandari S., Chambers S., Dweik R., Epton M., Analysis of biogenic volatile organic compounds in human health and disease. Trends Anal. Chem. 2011, 30, 960-967.

[15] Buszewski B., Kesy M., Ligor T., Amann T., Human exhaled air analytics: biomarker of disease. Biomed. Chromatogr. 2007, 21, 553-566.

[16] Yannick S., Wolff M., VOC breath biomarkers in lung cancer. Clin. Chim. Acta 2016, 459, 5-9. 
[17] Li J., Peng Y., Liu Y., Li W., Jin Y., Tang Z., Duan Y., Investigation of potential breath biomarkers for the early diagnosis of breast cancer using gas chromatography-mass spectrometry. Clin. Chim. Acta 2015, 436, 59-67.

[18] Fernández del Río R., O`Hara M. E., Holt A., Pemberton P., Shah T., Whitehouse T., Mayhew C. A., Volatile biomarkers in breath associated with liver cirrhosiscomparisons of pre- and post-liver transplant breath samples. EBioMedicine 2015, $2,1243-1250$.

[19] Mills G. A., Walker V., Headspace solid-phase microextraction profiling of volatile compounds in urine: application to metabolic investigations. J. Chromatogr. B: Biomed. Sci. Appl. 2001, 753, 259-268.

[20] Guernion N., Ratcliffe M., Spencer-Phillips P. T., Howe R. A., Identifying bacteria in human urine: current practice and the potential for rapid, near-patient diagnosis by sensing volatile organic compounds. Clin. Chem. Lab. Med. 2007, 39, 893-906.

[21] Aathithan S., Plant J. C., Chaudry A. N., French G. L., Diagnosis of bacteriuria by detection of volatile organic compounds in urine using an automated headspace analyzer with multiple conducting polymer sensors. J. Clin. Microbiol. 2001, 39, $2590-2593$.

[22] Banday K. M., Pasikanti K. K., Chan E. C. Y., Singla R., Rao K. V. S., Chauhan V. S., Nanda R. K., Use of urine volatile organic compounds to discriminate tuberculosis patients from heathy subjects. Anal. Chem. 2011, 83, 5526-5534.

[23] Batty C. A., Cauchi M., Hunter J. O., Woolner J., Baglin T., Tumer C., Differences in microbial metabolites in urine headspace of subjects with Immune Thrombocytopenia (ITP) detected by volatile organic compound (VOC) analysis and metabolomics. Clin, Chim. Acta 2016, 461, 61-68. 
[24] Silva C. L., Passos M., Câmara J. S., Investigation of urinary volatile organic metabolites as potential cancer biomarkers by solid-phase microextraction in combination with gas chromatography-mass spectrometry. Brit. J. Cancer 2011, $105,1894-1904$.

[25] Garner C. E., Smith S., Bardhan P. K., Ratcliffe N. M., Probert C. S. J., A pilot study of faecal volatile organic compounds in faeces from cholera patients in Bangladesh to determine their utility in disease diagnosis. Trans. R. Soc. Trop. Med. Hyg. 2009, 103, 1171-1173.

[26] Garner C. E., Smith S., Costello B. D., White P., Spencer R., Prober C. S. J., Ratcliffe N. M., Volatile organic compounds from feces and their potential for diagnosis of gastrointestinal disease. FASEB J. 2007, 21, 1675-1688.

[27] Khalid T., Aggio R., White P., Costello B. D. L., Persad R., Al-Kateb H., Jones P., Probert C. S., Ratcliffe N., Urinary volatile organic compounds for the detection of $\begin{array}{lllll}\text { prostate } & \text { cancer. } & P L O S & \text { ONE } & \text { 2015, }\end{array}$ doi:10.1371/journal.pone.0143283.

[28] Peng G., Hakim M., Broza Y. Y., Billan S., Abdah-Bortnyak R., Kuten A., Tisch U., Haick H., Detection of lung, breast, colorectal, and prostate cancers from exhaled breath using a single array of nanosensors. Brit. J. Cancer 2010, 103, 542551.

[29] Snow N. H., Bullock G. P., Novel techniques for enhancing sensitivity in static headspace extraction-gas chromatography. J. Chromatogr. A 2010, 1217, 27262735.

[30] Soria A. C., García-Sarrió M. J., Sanz M. L., Volatile sampling by headspace techniques. Trends Anal. Chem. 2015, 71, 85-99. 
[31] Calejo I., Moreira N., Araújo A. M., Carvalho M., Bastos M. L., Pinho P. G., Optimization and validation of a HS-SPME-GC-IT/MS method for analysis of carbonyl volatile compounds as biomarkers in human urine: Application in a pilot study to discriminate individuals with smoking habits. Talanta 2016, 148, 486-493.

[32] Kwak J., Grifsby C. C., Brittany R. S., Mateen M. R., Preti G., Changes in volatile compounds of human urine as it ages: their interaction with water. J. Chromatogr. B 2013, 941, 50-53.

[33] Scibetta L., Campo L., Mercadante R., Foà V., Fustinoni S., Determination of low level methyl tert-butyl ether, ethyl tert-butyl ether and methyl tert-amyl ether in human urine by HS-SPME gas chromatography/mass spectrometry. Anal. Chim. Acta 2007, 581, 53-62.

[34] González Paredes R. M., García Pinto C., Pérez Pavón J. L., Moreno Cordero B., Derivatization coupled to headspace programmed-temperature vaporizer gas chromatography with mass spectrometry for the determination of amino acids: Application to urine samples. J. Sep. Sci. 2016, 39, 3375-3383.

[35] Jaffè Ueber den Niederschlag M., welchen Pikrinsäure im normalen Harn-erzeugt und über eine neue Reaction des Kreatinins. Z. Physiol. Chem. 1886, 10, 391-400.

[36] Delanghe J. R., Speeckaert M. M., Creatinine determination according to Jaffè-what does it stand for? NDT Plus 2011, 4, 83-86. 
Page 17 of 24

\section{Journal of Separation Science}

385

386

387

388

389

390

391

392

393

394

395

\section{FIGURE CAPTIONS}

Fig. 1 Comparative study between acidic and physiological $\mathrm{pH}$.

Fig. 2 Optimization of a) sample volume and b) equilibration time.

Fig. 3 Comparison between the signals obtained with UHQ water and with urine samples from two subjects at a dilution ratio of 1:5.

Fig. 4 Chromatograms provided by a) a spiked UHQ water, b) a urine sample (nonspiked) and c) a spiked urine sample subjected to the optimized analytical procedure. The concentrations at which UHQ water and urine were spiked (chromatograms a and c) are shown in parentheses after the name of each compound 1. dimethyl disulfide $\left(10 \mu \mathrm{L} \mathrm{L}^{-1}\right)$; 2. toluene $\left(0.14 \mu \mathrm{L} \mathrm{L}^{-1}\right)$; 3. 1,4-xylene $\left(0.07 \mu \mathrm{L} \mathrm{L}^{-1}\right)$; 4. 2-octanone $\left(0.17 \mu \mathrm{L} \mathrm{L}^{-1}\right)$; 5. 2,6dimethyl-7-octen-2-ol $\left(0.22 \mu \mathrm{L} \mathrm{L}^{-1}\right)$. 
1 Table 1. Analytical characteristics of the method.

\begin{tabular}{|c|c|c|c|c|c|c|c|c|}
\hline \multirow[b]{2}{*}{ Compound } & \multirow{2}{*}{$\begin{array}{c}\text { Calibration } \\
\text { range } \\
(\mu \mathrm{g} / \mathrm{L})\end{array}$} & \multirow{2}{*}{$R^{2}$} & \multicolumn{2}{|c|}{ Repeatability (RSD \%) } & \multicolumn{2}{|c|}{ Reproducibility (RSD \%) } & \multirow{2}{*}{$\begin{array}{l}\text { LOD } \\
(\mu \mathrm{g} / \mathrm{L})\end{array}$} & \multirow{2}{*}{$\begin{array}{c}\text { LOQ } \\
(\mu \mathrm{g} / \mathrm{L})\end{array}$} \\
\hline & & & $\begin{array}{l}\text { Low level } \\
\text { (1 } \mu \mathrm{g} / \mathrm{L})\end{array}$ & $\begin{array}{l}\text { High level } \\
(10 \mu \mathrm{g} / \mathrm{L})\end{array}$ & $\begin{array}{c}\text { Low level } \\
(1 \mu \mathrm{g} / \mathrm{L})\end{array}$ & $\begin{array}{l}\text { High level } \\
(10 \mu \mathrm{g} / \mathrm{L})\end{array}$ & & \\
\hline Dimethyl disulfide & $1.5-50$ & 0.9989 & 9.5 & 6.7 & 11.1 & 8.5 & 0.48 & 1.43 \\
\hline Toluene & $0.1-50$ & 0.9995 & 2.2 & 5.0 & 9.7 & 9.9 & 0.02 & 0.07 \\
\hline 1,4-xylene & $0.1-50$ & 0.9992 & 2.3 & 4.7 & 7.2 & 8.0 & 0.01 & 0.05 \\
\hline 2-octanone & $0.2-50$ & 0.9929 & 3.6 & 6.7 & 14.2 & 10.6 & 0.06 & 0.17 \\
\hline 2,6-dimethyl-7-octen-2-ol & $0.1-50$ & 0.9956 & 3.6 & 7.3 & 7.4 & 7.0 & 0.03 & 0.08 \\
\hline
\end{tabular}


Page 19 of 24

Journal of Separation Science

1 Table 2. Method validation.

\begin{tabular}{lcccccc}
\hline \multirow{2}{*}{ Compound } & $\begin{array}{c}\text { Actual } \\
\text { concentration } \\
(\mu \mathrm{g} / \mathrm{L})\end{array}$ & Urine 1 & Urine 2 & Urine 3 & Urine 4 & Urine 5 \\
\cline { 3 - 7 } & 10 & $10 \pm 2$ & $11 \pm 2$ & $11 \pm 1$ & $10 \pm 1$ & $8 \pm 2$ \\
Dimethyl disulfide & 1.0 & $0.8 \pm 0.2$ & $0.9 \pm 0.2$ & $0.9 \pm 0.1$ & $1.1 \pm 0.2$ & $1.0 \pm 0.2$ \\
Toluene & 1.0 & $1.0 \pm 0.2$ & $1.4 \pm 0.4$ & $1.0 \pm 0.2$ & $1.3 \pm 0.3$ & $1.4 \pm 0.4$ \\
1,4-xylene & 1.0 & $0.8 \pm 0.2$ & $1.0 \pm 0.2$ & $0.9 \pm 0.1$ & $0.9 \pm 0.1$ & $0.9 \pm 0.2$ \\
2-octanone & 1.0 & $1.2 \pm 0.2$ & $1.4 \pm 0.4$ & $1.1 \pm 0.2$ & $1.0 \pm 0.2$ & $1.1 \pm 0.2$ \\
2,6-dimethyl-7-octen-2-ol & & & & &
\end{tabular}


1 Table 3. Results obtained for the compounds studied in urine samples.

\begin{tabular}{lc|c|c|c|c|c}
\hline \multirow{2}{*}{ Compound } & \multicolumn{2}{c}{ Urine 1} & \multicolumn{2}{c}{ Urine 2 } & \multicolumn{2}{c}{ Urine 3 } \\
\cline { 2 - 8 } & $\mu \mathrm{g} / \mathrm{L}$ & $\begin{array}{l}\mathrm{nmol} \text { analyte/ } \\
\mathrm{mmol} \text { creatinine }\end{array}$ & $\mu \mathrm{g} / \mathrm{L}$ & $\begin{array}{l}\mathrm{nmol} \text { analyte/ } \\
\mathrm{mmol} \text { creatinine }\end{array}$ & $\mu \mathrm{g} / \mathrm{L}$ & $\begin{array}{l}\mathrm{nmol} \text { analyte/ } \\
\mathrm{mmol} \text { creatinine }\end{array}$ \\
\hline Dimethyl disulfide & $7 \pm 1$ & $5.5 \pm 0.8$ & $5 \pm 1$ & $3.9 \pm 0.8$ & $8 \pm 1$ & $5.7 \pm 0.7$ \\
Toluene & $0.14 \pm 0.01$ & $0.113 \pm 0.008$ & $0.13 \pm 0.01$ & $0.103 \pm 0.008$ & $0.18 \pm 0.02$ & $0.13 \pm 0.01$ \\
1,4-xylene & $0.056 \pm 0.006$ & $0.039 \pm 0.004$ & $0.051 \pm 0.006$ & $0.035 \pm 0.004$ & $0.07 \pm 0.01$ & $0.044 \pm 0.006$ \\
2-octanone & $<\mathrm{LOQ}$ & $<\mathrm{LOQ}$ & $<\mathrm{LOQ}$ & $<\mathrm{LOQ}$ & $<\mathrm{LOQ}$ & $<\mathrm{LOQ}$ \\
\hline 2,6-dimethyl-7-octen-2-ol & $0.103 \pm 0.007$ & $0.049 \pm 0.003$ & $0.13 \pm 0.01$ & $0.061 \pm 0.005$ & $0.20 \pm 0.02$ & $0.085 \pm 0.009$ \\
\hline
\end{tabular}




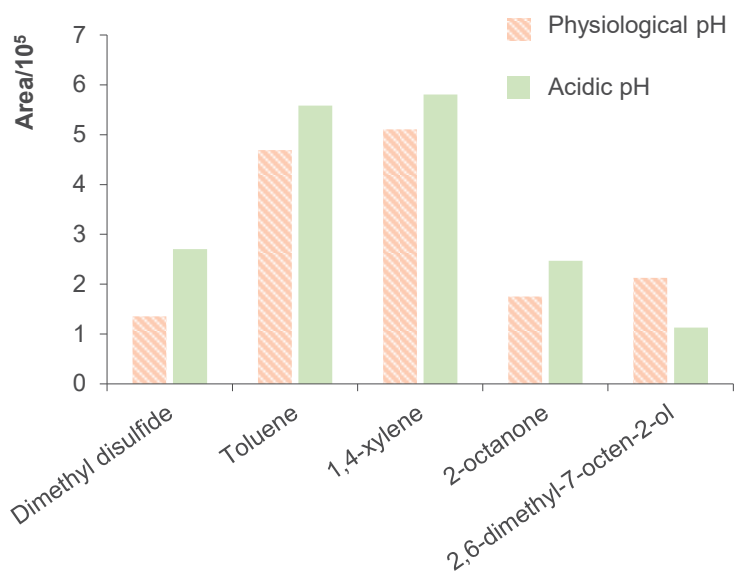


a

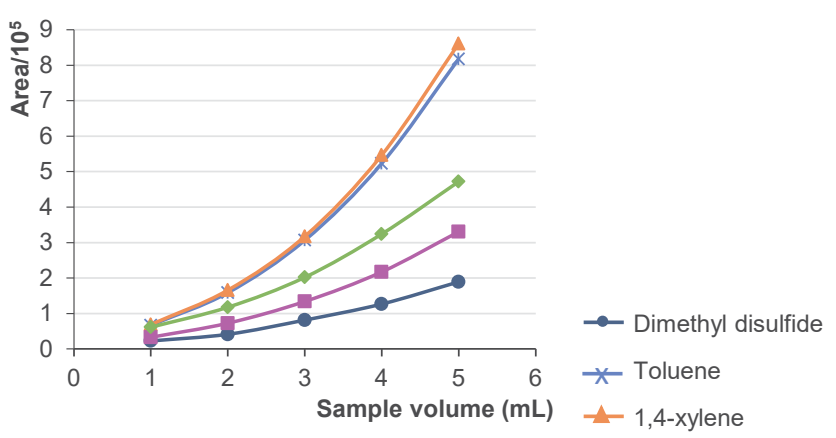

b

$\rightarrow$ 2-octanone

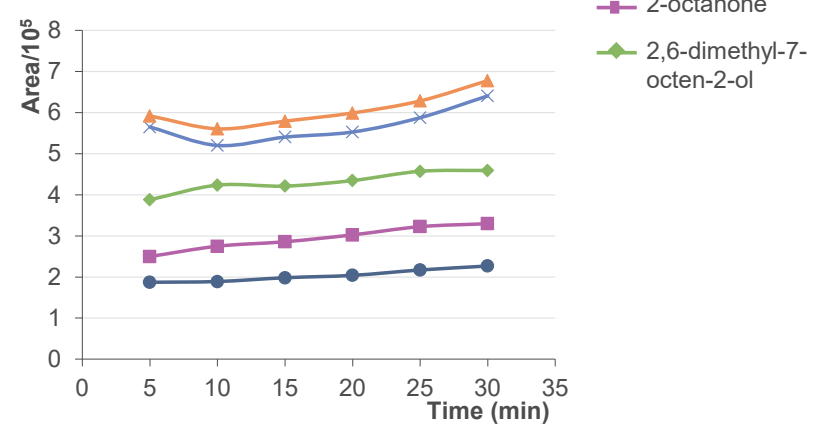

Wiley-VCH 


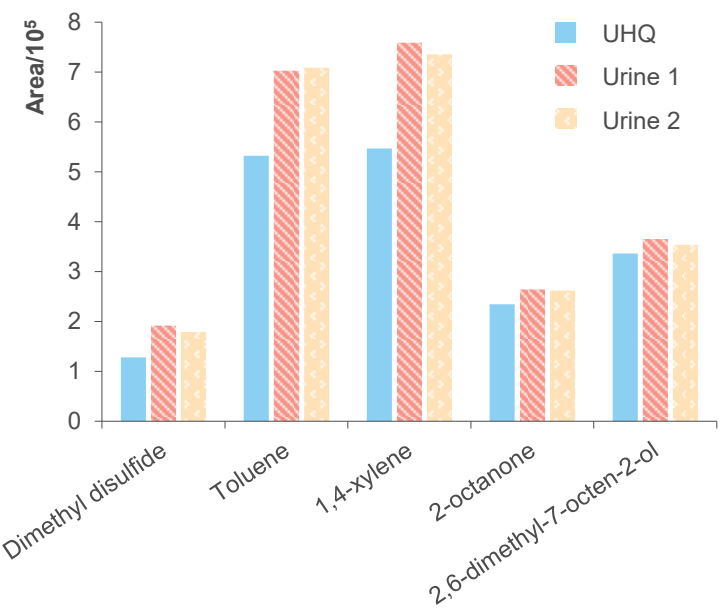


a) Spiked UHQ water
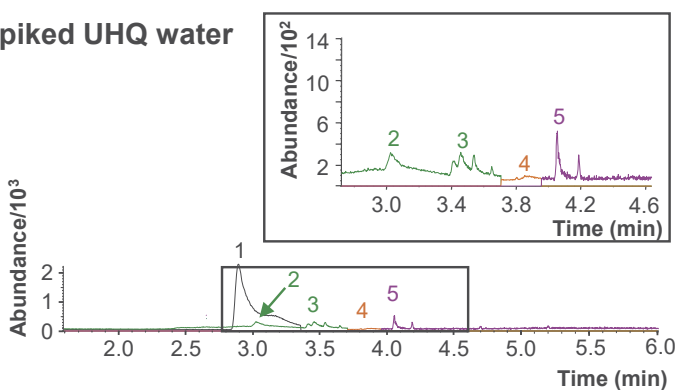

b) Urine sample

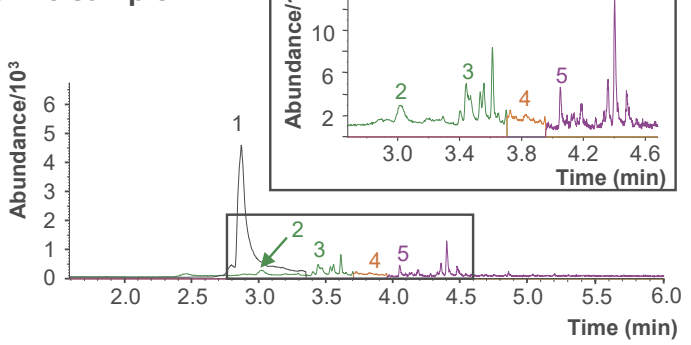

c) Spiked urine sample

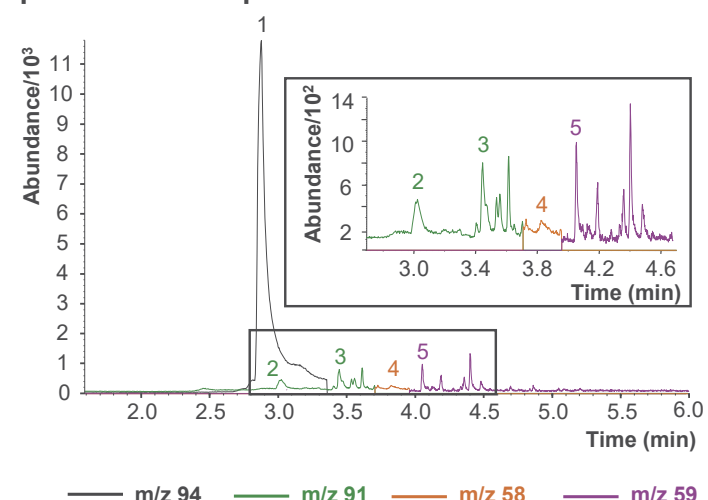



CONCLUSIONES 

En la presente memoria se han propuesto nuevas metodologías analíticas encaminadas a conseguir una reducción significativa del esfuerzo necesario para llevar a cabo la etapa de tratamiento de muestra con el fin de minimizar costes y tiempo y reducir los errores asociados a esta etapa del proceso analítico.

Con el fin de estudiar las posibilidades de estas nuevas metodologías, se han puesto a punto diferentes métodos analíticos destinados a la determinación de compuestos de interés biológico y medioambiental en distintos tipos de matrices.

A continuación, se exponen las principales conclusiones obtenidas, unas de carácter general, referidas a las metodologías utilizadas y otras particulares de las diferentes aplicaciones que se han desarrollado.

$>$ Estudio de las posibilidades de las columnas capilares con fases estacionarias de líquidos iónicos en cromatografía de gases.

El uso de columnas de líquidos iónicos, que presentan diferentes polaridades, puede facilitar la separación cromatográfica de compuestos difícilmente separables mediante el uso de columnas convencionales con baja polaridad.

En el trabajo incluido en esta memoria se han evaluado cuatro columnas capilares de líquidos iónicos para la separación de clorobencenos. La columna SLB-IL100 da lugar a una pobre separación de los compuestos, mientras que la SLB-IL59 y la SLB-IL61 sufrieron un importante proceso de degradación que provocaba la pérdida de resolución. La columna más adecuada y que 
mejor permite la separación y cuantificación de los clorobencenos es la SLBIL82, ya que es la que proporciona los mejores valores de resolución y factor de asimetría. La comparación de esta columna de líquido iónico con la columna convencional HP5, muestra que el perfil de elución es diferente y que la columna SLB-IL82 proporciona una mejor separación de los compuestos, siendo ésta, por tanto, la seleccionada para la propuesta de un método analítico para la determinación de clorobencenos en suelos.

\section{> Acoplamiento de la derivatización y extracción in situ con la técnica MEPS para la preconcentración y determinación de clorofenoles.}

A diferencia de la mayoría de las metodologías propuestas para la determinación de clorofenoles en suelos en las que primero se realiza la extracción de los clorofenoles del suelo y posteriormente se lleva a cabo la derivatización de los mismos, en la metodología propuesta en esta memoria se realiza la derivatización de los clorofenoles directamente en el suelo y se extraen del suelo los compuestos ya derivatizados. Además de reducir el tiempo empleado en el pretratamiento de muestra al realizar la derivatización y extracción en un solo paso, el poder llevar a cabo la derivatización en medio acuoso facilita en gran medida su acoplamiento con MEPS, puesto que se dispone directamente y sin necesidad de ninguna etapa adicional, de un extracto acuoso con los compuestos derivatizados listo para ser sometido al proceso MEPS.

La técnica MEPS reduce, por un lado, el volumen de muestra necesario para llevar a cabo la preconcentración de los analitos, frente a los utilizados en otras técnicas de extracción y, por otro, el volumen de disolvente orgánico 
con el que se lleva a cabo la elución de los compuestos retenidos. Además, la utilización del MEPS automático acoplado en línea con el cromatógrafo de gases permite realizar el proceso de forma automática, lo que supone un considerable ahorro de tiempo de análisis.

La estrategia propuesta consiste, por tanto, en dos partes. La primera es una etapa manual en la que se lleva a cabo la derivatización y extracción in situ de los clorofenoles de las muestras de suelos en un solo paso. La segunda se trata de un procedimiento totalmente automático en el que el vial con el extracto acuoso obtenido de la etapa anterior se coloca en el automuestrador del equipo y se lleva a cabo la preconcentración y análisis de los compuestos mediante MEPS-PTV-GC-MS. Este acoplamiento, por tanto, simplifica enormemente el tratamiento de muestra y reduce tanto el consumo de reactivos como el tiempo de análisis.

\section{> Acoplamiento de la derivatización con la generación de espacio de cabeza para la determinación de aminoácidos.}

La utilización de la reacción de derivatización de aminoácidos con cloroformiato de etilo en el propio vial de generación de espacio de cabeza ha demostrado ser una alternativa eficaz para el aumento de sensibilidad en el acoplamiento HS-GC.

Con la utilización de este tipo de reacciones, en las cuales se generan derivados más volátiles que los analitos originales, se puede ampliar el grupo de compuestos que pueden ser analizados por HS, incluyendo aquellos que, en principio, no podrían serlo, como consecuencia de su baja volatilidad. Además, al reducir la polaridad de los analitos, se produce una mejora de las 
características cromatográficas, tales como reproducibilidad, sensibilidad y morfología de pico.

La utilización de esta configuración instrumental tiene la ventaja de que, desde la mezcla de los reactivos en el vial de HS, todo el proceso tiene lugar en continuo, con la consecuente reducción de errores asociados a la manipulación de muestra. Además, este acoplamiento permite eliminar el uso de disolventes o de otros tratamientos para extraer los compuestos derivatizados de la matriz antes de su inyección en GC.

\section{> Utilización de la técnica de generación de espacio de cabeza para la determinación de compuestos volátiles en orina.}

Se han comprobado las ventajas que supone la utilización de la técnica de pretratamiento de muestra de generación de espacio de cabeza para el análisis de compuestos volátiles en muestras de orina, como la facilidad de preparación de muestra y la facilidad de implementación y automatización. Esta técnica permite el análisis de compuestos de interés presentes en estas matrices separándolos de otros compuestos interferentes no volátiles sin tener que recurrir a etapas de pretratamiento adicionales.

Se ha propuesto una metodología para la determinación en muestras de orina de compuestos volátiles con diferentes grupos funcionales y volatilidades descritos como posibles biomarcadores, que utiliza la generación de espacio de cabeza estático como técnica de pretratamiento y en la que no se lleva a cabo ninguna etapa de preconcentración adicional. Esto hace que el procedimiento final sea simple y rápido, lo que, unido a la capacidad de determinar conjuntamente compuestos de diferentes familias 
químicas y volatilidades, hace de este método una estrategia adecuada para futuros estudios en los que se analicen un gran número de muestras y que lleven al descubrimiento de nuevos biomarcadores o a la confirmación de la utilidad de aquellos ya descritos. 



\section{VERSIÓN RESUMIDA EN INGLÉS SUMMARY IN ENGLISH}





\section{TABLE OF CONTENTS}

\section{INTRODUCTION AND AIMS}

\section{CHAPTER I. DEVELOPMENT OF ANALYTICAL METHODOLOGIES FOR THE DETERMINATION OF COMPOUNDS OF ENVIRONMENTAL INTEREST IN SOIL SAMPLES}

I.1. Ionic liquids as stationary phases in gas chromatography. Determination of chlorobenzenes in soils

I.2. In situ derivatization combined to automated microextraction by packed sorbent for the determination of chlorophenols in soil samples by gas chromatography mass spectrometry

Journal of Chromatography A 1359 (2014) 52-59

CHAPTER II. DEVELOPMENT OF ANALYTICAL METHODOLOGIES FOR THE DETERMINATION OF COMPOUNDS OF BIOLOGICAL INTEREST IN URINE SAMPLES

II.1. Derivatization coupled to headspace programmedtemperature vaporizer gas chromatography with mass spectrometry for the determination of amino acids. Application to urine samples

Journal of Separation Science 39 (2016) 3375-3383

II.2. Determination of possible biomarkers in urine samples by a rapid method based on headspace-gas chromatographymass spectrometry 



\section{Introduction}

The presented Doctoral Thesis focuses on the development of analytical methodologies based on the use of gas chromatography (GC) as separation technique and directed to achieving a significant reduction of the effort needed to perform the sample treatment step with the purpose of minimizing costs and analysis time and reducing errors associated to this step of the analytical process.

The Doctoral Thesis consists of two parts, both dedicated to the development of different methodologies that meet the characteristics above described but differ in the type of matrix of study. The first part is dedicated to soil matrices and the second one to biological matrices.

The first part focuses on the development of analytical methodologies for the determination of contaminants present in soil samples, in particular chlorobenzenes and chlorophenols, while the second part focuses on the development of rapid and simple methodologies for the detection and quantification of compounds of biological interest in urine, such as amino acids and some compounds described as possible biomarkers. 
Given the diversity of the type of matrices and of the nature of the compounds studied, the optimization and development of rapid analytical methods for their determination entails employing a variety of sample pretreatment techniques.

The first work included in the thesis approaches the determination of chlorobenzenes in soils. Their extraction of the matrix by means of simplified quick, easy, cheap, effective, rugged and safe (QuEChERS) extraction already reported [1] is optimized. This simplified version of the QuEChERS method eliminates the dispersive solid phase extraction (d-SPE) cleanup step after extraction due to the nonfatty characteristics of soil matrices. In addition, in this work, an evaluation of the behavior of the new capillary columns with stationary phases of ionic liquids (ILs) for the chromatographic separation of chlorobenzenes is carried out. Research on ionic liquids (organic salts with low melting points) has experienced a great increase in recent years and some of their special characteristics [2] such as low volatility, stability to high temperature, absence of hydroxyl groups and the possibility of synthesizing them with the desired selectivity have made them interesting for their study as stationary phases in gas chromatography. The ILs columns used in this work were supplied by Supelco through SigmaAldrich within the SILEP (Supelco Ionic Liquid Evaluation Program) international project.

For the determination of chlorophenols in soils, automated microextraction by packed sorbent (MEPS) is used to preconcentrate the compounds. MEPS is a developed technique that arises from the attempt to speed up and make more efficient the solid-phase extraction (SPE) [3,4]. It is 
a sorption-based separation technique for sample preparation based on the miniaturization and automation of conventional SPE in which a sorbent packed material (ca. 1-4 mg) is placed at the top of a syringe needle. This technique not only reduces the consumption of organic solvents but also allows reusing the MEPS cartridges a large number of times [5].

In gas chromatography, the derivatization reactions are used extensively for the improvement of the chromatographic characteristics of some compounds. This is the case of chlorophenols studied in this work, for which an acylation reaction with acetic anhydride in basic medium directly in the soil sample is used. This derivatization in aqueous medium is a great advantage when MEPS is used after it.

Biological samples are a valuable information source on the health status of an individual since the presence of certain organic compounds in them is related to some basic cellular functions [6]. For this reason, the development of new analytical methods for the determination of compounds in biological samples, as is performed in the second part of the thesis, constitutes a necessary and interesting line of study not only for the research and discovery of new biomarkers $[7,8]$ that can be used for the early detection of some diseases, but also as a tool to evaluate the usefulness of the ones previously proposed.

In the first work of this second part of the thesis, in which a method for the determination on amino acids in urine samples is proposed, a derivatization reaction is used again. In this case ethyl chloroformate (ECF) is employed as derivatization reagent of amino acids. This derivatization reaction is coupled to headspace sampling (HS), so that the volatile 
derivatized compounds are injected directly into the chromatographic system without the need to carry out additional treatments to extract them from the matrix.

In the second work of this part of the thesis, the headspace sampling is still used as a pretreatment technique for the determination in urine samples of compounds belonging to different chemical families and with different volatilities that have been described as possible biomarkers. This technique of headspace generation for the analysis of volatile biomarkers in biological matrices not only minimizes the treatment required by this type of samples [9] but also allows the automation of the process, and reduces the interference of non-volatile compounds present in complex matrices [10]. Thus, a fast, simple and sensitive method capable of determining compounds with different functional groups and that can be applied to the analysis of a large number of samples for the discovery and confirmation of biomarkers is achieved.

\section{Objectives and working hypothesis}

The general and main objective of this Doctoral Thesis is to develop rapid analytical methodologies that simplify the sample treatment and allow the determination and quantification of different compounds of biological and environmental interest in different types of matrices.

The specific objectives for the first part of the thesis dedicated to the determination of contaminant in soils are fundamentally: 
The study of the potential advantages of the new ionic liquid stationary phases for the determination of chlorobenzenes.

The study of the analytical possibilities of the coupling of in situ derivatization and extraction with MEPS for the preconcentration and determination of chlorophenols.

The hypotheses laid out in this first part are:

- The first one, that the simplified version of QuEChERS allows the extraction of chlorobenzenes from soil samples.

- The second one, that new ionic liquid stationary phases, more polar than conventional ones, allow adequate separation of chlorobenzenes, considering that the chromatographic characteristics will differ depending on the ionic liquid that constitutes the stationary phase.

- The third one, that the derivatization and extraction of chlorophenols can be carried out in situ in the soil samples.

- The fourth one, that the microextraction technique MEPS allows the preconcentration of chlorophenols once all the variables involved in it have been optimized.

- The fifth one, that the coupling of the in situ derivatization and extraction with MEPS for the determination of chlorophenols is feasible. 
The specific objectives of the second part dedicated to biological matrices are:

$>$ The development of a series of rapid, sensitive and efficient analytical methodologies applicable to the determination and quantification in urine samples of some compounds described as biomarkers.

The study of the headspace technique for the determination of volatile compounds in urine samples.

The study of the coupling of the derivatization with the headspace technique for the determination of amino acids.

In this part, the hypotheses laid out are:

- The first one, that the derivatization of amino acids can be carried out in the urine samples.

- The second one, that the coupling of the derivatization with the headspace technique for the determination of amino acids is feasible.

- The third one, that the headspace generation technique (HS) is suitable for the determination of volatile compounds in urine as it reduces the treatment to which the sample should be subjected and eliminates many non-volatile interfering compounds present in this type of matrices. 


\section{References}

[1] C. García Pinto, M.E. Fernández Laespada, S. Herrero Martín, A.M. Casas Ferreira, J.L. Pérez Pavón, B. Moreno Cordero, Simplified QuEChERS approach for the extraction of chlorinated compounds from soil samples, Talanta 81 (2010) 385-391.

[2] C.F. Poole, S.K. Poole, Ionic Liquid stationary phases for gas chromatography, J. Sep. Sci. 34 (2011) 888-900.

[3] M. Abdel-Rehim, New trend in sample preparation: on-line microextraction in packed syringe for liquid and gas chromatography applications. I. Determination of local anaesthetics in human plasma samples using gas chromatography-mass spectrometry, J. Chromatogr. B 801 (2004) 317-321.

[4] A. El-Beqqali, A. Kussak, M. Abdel-Rehim, Fast and sensitive environmental analysis utilizing microextraction in packed syringe online with gas chromatography-mass spectrometry: determination of polycyclic aromatic hydrocarbons in water, J. Chromatogr. A 1114 (2006) 234-238.

[5] A.R. Chaves, F.Z. Leandro, J.A. Carris, M.E.C. Queiroz, Microextraction in packed sorbent for analysis of antidepressants in human plasma by liquid chromatography and spectrophotometric detection, J. Chromatogr. B 878 (2010) 2123-2129.

[6] I. Horváth, Z. Lázár, N. Gyulai, M. Kollai, G. Losonczy, Exhaled biomarkers in lung cancer, Eur. Respir. J. 34 (2009) 261-275. 
[7] H.J. Issaq, T.J. Waybright, T.D. Veenstra, Cancer biomarker discovery: Opportunities and pitfalls in analytical methods, Electrophoresis 32 (2011) 967-975.

[8] K.A. Kouremenos, M. Johansson, P.J. Marriott, Advances in gas chromatographic methods for the identification of biomarkers in cancer, J. Cancer 3 (2012) 404-420.

[9] B. Kolb, in I.D. Wilson (Editor), CHROMATOGRAPHY: GAS Headspace Gas Chromatography. Encyclopedia of Separation Science. Academic Press, Oxford, 2000, pp. 489-496.

[10] N.H. Snow, G.P. Bullock, Novel techniques for enhancing sensitivity in static headspace extraction-gas chromatography, J. Chromatogr. A 1217 (2010) 2726-2735. 


\section{Conclusions}

In the present Doctoral Thesis, new analytical methodologies have been proposed, aimed at achieving a significant reduction on the effort necessary to carry out the sample treatment step, as well as at minimizing costs and analysis time and diminishing errors associated with this stage of the analytical process.

To study the possibilities of these new methodologies, different analytical methods for the determination of compounds of biological and environmental interest in different matrices have been developed.

The main conclusions are outlined below. Some are general conclusions and others are particular ones of the different applications developed.

Study of the potential of capillary columns with ionic liquids as stationary phases in gas chromatography.

The use of columns with stationary phases of ionic liquids that present different polarities can facilitate the chromatographic separation of 
compounds that are difficult to separate using conventional low polarity columns.

In the work included in this Doctoral Thesis, four capillary columns of ionic liquids have been evaluated for the separation of chlorobenzenes. The SLB-IL100 column does not afford an appropriate separation of the compounds, whereas SLB-IL59 and SLB-IL61 undergo an important degradation process leading to a significant loss of resolution. The most suitable column for their separation and quantification is the SLB-IL82, since it provides the best values of resolution and asymmetry factor. The comparison of this ionic liquids column with the conventional HP5 column shows that the elution profile is different and that the SLB-IL82 column provides better separation of the compounds. Therefore, this last column was selected to propose an analytical method for the determination of chlorobenzenes in soil samples.

\section{$>$ Coupling of in situ derivatization and extraction with the MEPS technique for the preconcentration and determination of chlorophenols.}

Unlike most of the methodologies proposed for the determination of chlorophenols in soils in which the extraction of chlorophenols is carried out first and then their derivatization is performed, in the methodology proposed herein the derivatization of chlorophenols is carried out directly in the soil and the already derivatized compounds are extracted from the soil. In addition to reducing the sample pretreatment time by performing derivatization and extraction in a single step, carrying out the derivatization in aqueous media greatly facilitates its coupling with MEPS, since an aqueous extract with the derivatized compounds ready to be subjected to the 
MEPS process is available directly, and without the need of any additional step.

The MEPS technique reduces, firstly, the sample volume necessary to carry out preconcentration of analytes compared with those used in other extraction techniques and, secondly, the volume of organic solvent that is used for the elution of the retained compounds. Moreover, the use of automated MEPS coupled on-line with gas chromatography allows the process to be automated, which means a considerable saving of analysis time.

The proposed strategy, therefore, consists of two parts. The first one is a manual step in which the in situ derivatization and extraction of chlorophenols from the soil sample are carried out. The second is a completely automated procedure in which the vial with the aqueous extract obtained from the previous step is placed in the autosampler and the preconcentration and analysis of the compounds are carried out by means of MEPS-PTV-GC-MS. This coupling of in situ derivatization and extraction with MEPS greatly simplifies sample treatment and reduces both reagent consumption and analysis time.

$>$ Coupling of the derivatization with the headspace sampling technique for the determination of amino acids.

The use of the amino acid derivatization reaction with ethyl chloroformate in the headspace vial has proved to be an effective alternative for increased sensitivity in HS-GC analysis.

With the use of this type of reactions in which compounds more volatile than the original analytes are generated, the group of compounds 
that can be analyzed by HS can be extended to low volatility compounds which initially could not be transferred to the headspace. Additionally, with the formation of less polar compounds, an improvement of the chromatographic characteristics (such as reproducibility, sensibility and peak shape) is observed.

This instrumental configuration has the advantage that, as from the mixing of the reagents in the HS vial, the whole process takes place on-line, with the consequent reduction in errors associated with sample manipulation. Furthermore, this coupling allows eliminating the use of solvents or additional pretreatments to isolate the derivatives from the matrix before their injection in the GC system.

\section{$>$ Use of headspace sampling technique for the determination of volatile compounds in urine samples.}

The advantages of the use of headspace sampling as pretreatment technique for the analysis of volatile compounds in urine samples, such as ease of sample preparation and ease of implementation and automation, have been checked. This technique allows the analysis of the compounds of interest in this matrix by separating them from other non-volatile interfering compounds without having to resort to additional pretreatment steps.

Specifically, a methodology using the headspace sampling as pretreatment technique of urine samples and in which no additional preconcentration step is used has been proposed for the determination of possible biomarkers with different functional groups and volatility. The final 
procedure is simple and fast and allows determining jointly compounds from different chemical families. These characteristics make this method an appropriate strategy for further studies in which a large number of samples can be analyzed and can lead to the discovery of new biomarkers or to the confirmation of the usefulness of the ones previously described. 



\section{APÉNDICE: MENCIÓN “DOCTORADO INTERNACIONAL"}



Con la elaboración y defensa de esta memoria de tesis se pretende optar a la obtención de la mención "Doctor Internacional" en el título de Doctor por la Universidad de Salamanca, al considerar que la doctoranda reúne los requisitos para optar a tal mención:

- La doctoranda ha realizado una estancia de tres meses en el Department of Environmental Chemistry and Bioanalytics, Faculty of Chemistry, Nicolaus Copernicus University, en Toruń (Polonia), relacionada con la presente tesis.

- Esta memoria incluye un resumen en inglés donde se detallan los objetivos y conclusiones de la misma.

- La tesis cuenta con los informes favorables de dos doctores expertos pertenecientes a instituciones de educación superior no españolas.

- Uno de los miembros que ha de formar parte del tribunal evaluador de la tesis es un experto perteneciente a un centro de investigación de prestigio no español.

Se incluyen, en este apéndice, la certificación de la estancia breve, así como los pertinentes informes favorables. 



\section{PROGRAMA DE MOVILIDAD DE PERSONAL INVESTIGADOR MODALIDAD Ib}

CERTIFICADO DEL CENTRO RECEPTOR TRAS LA ESTANCIA DE INVESTIGACIÓN CERTIFICATE OF STAY IN A FOREING INSTITUTION

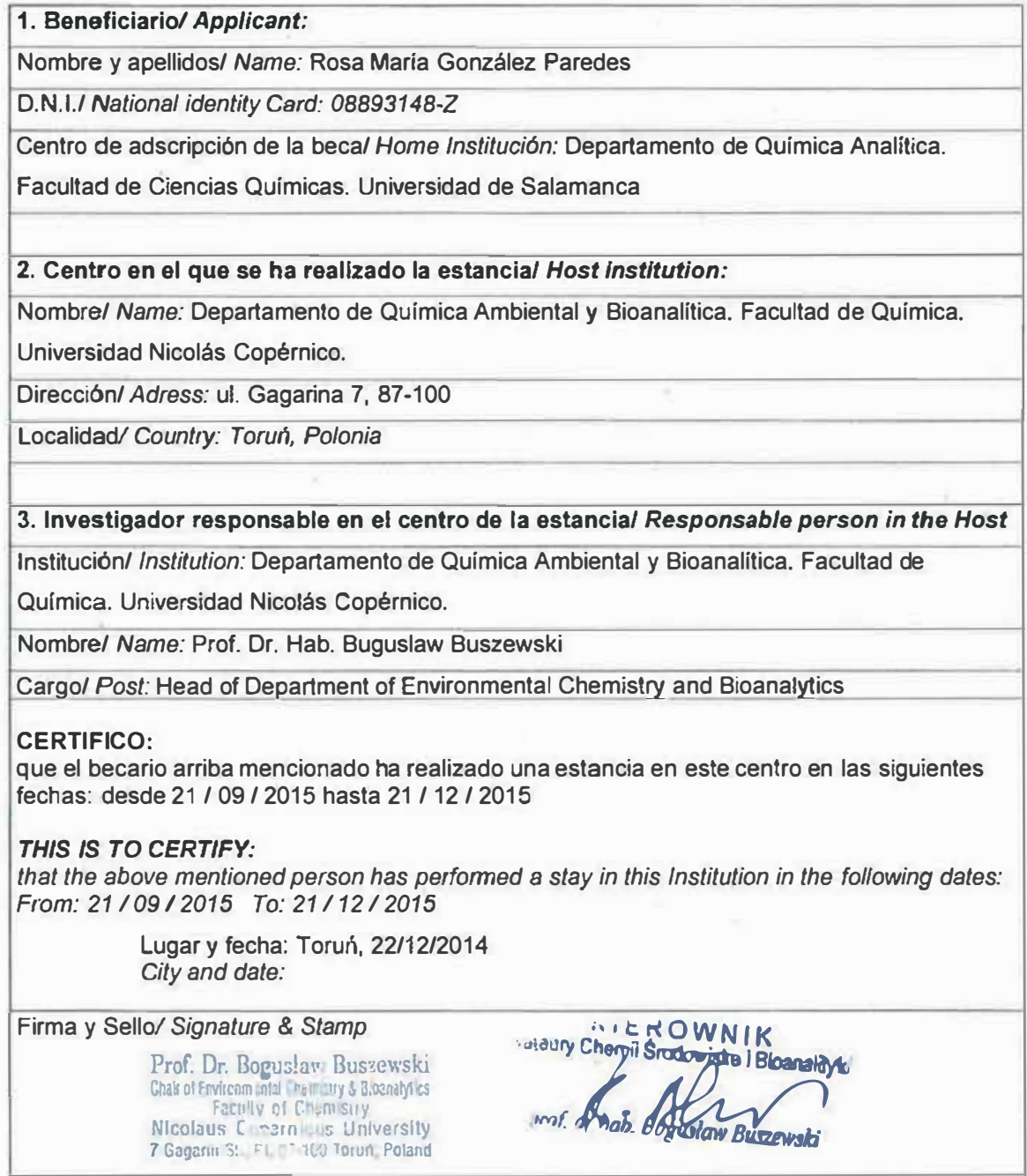




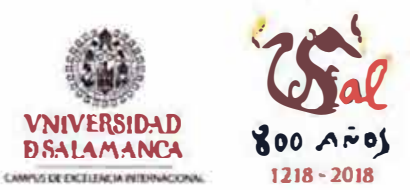

ESCVEA DE DOCTORADO

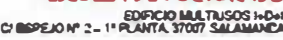
DSALAMANCA $1218-2018$

MODELO DE INFORME DE MENCIÓN DOCTOR INTERNACIONAL

STANDARD FORM FOR THE INTERNATIONAL DOCTORATE MENTION

NOMBRE Y APELLIDOS / NAME OF THE EXAMINER

Magdalena Ligor

CATEGORIA ACADENECA O PROFESIONAL ACADEMIC OR PROFESIONAL RANK

DSc

UNIVERSIDAD U ORGWNISMO Y PAIS / UNIVERSTY OR INSTIUTION AND COUNTRY

Nicolaus Copernicus University, Poland

TITULO DELU TZIS OQUETO DE INFORME / TILE OF THE TESIS

Development of new analytical strategies for the determination of compounds of biological and environmental interest

\section{MOMBRE Y APEWIDOS DEL DOCTORANDO I MAME OF THE CANDIDATE}

Rosa Maria Gonzàlez Paredes

NOMBRE Y APEWDOS DEL DIRECTORYA(S) DE LA TESIS / MAME OF THE THESIS DIRECTOR(S)

Dr. Garcia Pinto, Associate Professor of Analytical Chemistry

at the University of Salamanca 
INFORME RAZONADO/REASONEO REPORT

SOBRE LA CAUDAD CIENTIFICA OE IA TESAS DOCTORAL (puede oñodir los hojos que creo necesonias odjuntondo o este informe)

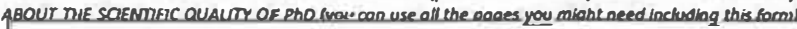

The presented PhD thesis comprises two main chapters that concern analytical methods developed for the determination of volatile and semivolatile compounds in environmental and human biological samples, based on using of gas chromatography (GC).

The introduction presents the general objectives of the thesis, especially analytical methodologies aimed to achieving a significant reduction on the effort necessary to carry out the sample treatment step as well as at ninimizing costs and analysis time and diminishing errors associated with this stage of the analytical process. The particular objectives are clear and well described.

The first chapter focuses on the analytical methods which have been developed for the detenmination of contaminants present in soil samples, such as chlorobenzenes and chlorophenols. The application of ionic liquids (ILS) as stationary phase for GC as well as extraction procedures including the simplified version of the QuEChERS method which eliminates some disadvantages of the dispersive solid phase extraction ( $d-S P E$ ) have been presented. It was proved that the use of columns with II.s stationary phases can facilitate the separation of compounds that are difficult to separate using conventional low polarity columns. Two methods - a manual procedure in which the in situ derivatization and extraction of chlorophenols from the soil sample and an automated one performed by means of MEPS-PN GC-MS - have been compared.

The second chapter presents a method for the determination of amino acids in urine samples which requires derivatization with ethyl chloroformate (ECF). This derivatization reaction has been coupled to headspace sampling (HS). In this way the derivatized compounds are injected directly into a GC system and no additional treatments are needed to extract them from the matrix. Moreover, this part presents the use of headspace sanpling which requires programmed temperature vaporization (PTV) as a pretreatment method to separate compounds from urine samples belonging to different chemical families that have been described as possible biomarkers. This method offers fast, simple and sensitive technique capable to determine compounds with various functional groups, and it can be applied in the analysis of numerous biological samples in order to discover and confirm the presence of biomarkers.

In conclusion, this PhD thesis presents an original work of high scientific and social impact. Therefore, based on its novelty and significance, I consider and recommend this thesis to be defended in front of a committee and is worthy of being awarded the International Doctor Mention.

Informo que la calidad científica de esta Tesls es merecedora de obtener la Mencion Doctor Intemaciona (marcar con una cruz una opción)

I inform that the scientific quality of this thesis is worthy of obtalning the Intemational Doctor Mention (mark with across one option)

$$
\text { Favorable } \mathrm{X}
$$

fecha / dATE: Aprie 13,2017

FIRMA / SIGNATURA original: thagdaleme ofifor

SELO DE LA INSTITUCIÓN DEL FIRMANTE / THE INSTITUTION'S STAMP OF THE PERSON WHO FIRMS

Uniwersytet Mikołaja Kopernikfágina 2 do 2 WYDZIAL CHEMII

Kalodro Chermil Srodowiska i Bioanelityty

87.100 Torunt, ul. Gagarina 7

(el.(056) 6114308 . for (n56) 6114837 


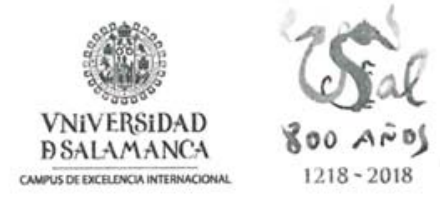

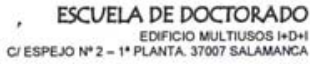
PSALAMANC.A 1218-2018

MODELO DE INFORME DE MENCIÓN DOCTOR INTERNACIONAL.

STANDARD FORM FOR THE INTERNATIONAL: DOCTORATE MENTION

NOMBRE Y APELLIDOS / NAME OF THE EXAMINER

ANGEL LUIS FUENTES DE ARRIBA

CATEGORÍA ACADÉMICA O PROFESIONAL/ ACADEMIC OR PROFESIONAL RANK

POSTDOCTORAL RESEARCHER

UNIVERSIDAD U ORGANISMO Y PAIS / UNIVERSITY OR INSTITUTION AND COUNTRY

UNIVERSITY OF OXFORD, UNITED KINGDOM

TITULO DE LA TESIS OBJETO DE INFORME / TITLE OF THE TESIS

DEVELOPMENT OF NEW ANALYTICAL STRATEGIES FOR THE DETERMINATION OF COMPOUNDS OF BIOLOGICAL AND ENVIRONMENTAL INTEREST

NOMBRE Y APELLIDOS DEL DOCTORANDO / NAME OF THE CANDIDATE

ROSA MARIA GONZALEZ PAREDES

NOMBRE Y APELLIDOS DEL DIRECTOR/A(S) DE LA TESIS / NAME OF THE THESIS DIRECTOR(S)

PROF. BERNARDO MORENO CORDERO

PROF. JOSE LUIS PEREZ PAVON

PROF. CARMELO GARCIA PINTO 
INFORME RAZONADO/REASONED REPORT

SOBRE LA CALIDAD CIENTIFICA DE LA TESIS DOCTORAL (puede añadir las hojas que crea necesarias adjuntando a este informe) ABOUT THE SCIENTIFIC QUALITY OF PhD (you can use all the pages you might need including this form

The thesis presented by the PhD Rosa Maria Gonzalez Paredes constitutes' an excellent research project. The work carried out by the candidate represents cutting-edge science within analytical strategies for the determination of compounds of biological and environmental interest.

The $\mathrm{PhD}$ thesis is structured in a general introduction, two chapters, each one consists of two research projects and ends with conclusions and a summary in English in order to obtain the International Doctorate Mention.

The document is perfectly structured, the aims and work hypothesis of the project are perfectly explained, all the objectives are presented concisely and in a clear way and have been successfully achieved and the introduction gives a clear overview of the different techniques and instrumental configuration used by the candidate.

The thesis is well written and the content is well organized and logically presented.

The results obtained are successful and have been published in high rank international journals, so, the quality of the work has already been reviewed by international referees who guarantee the exceptional labour carried out by the candidate Gonzalez Paredes.

The work developed in this thesis is really interesting, not only regarding its formative character, which has allowed the candidate to acquire knowledge and hands-on experience in modern and cutting-edge analytical techniques, but also for the interesting applications which can arise from this work, which makes the determination of contaminants in soil samples and biomarkers in biological samples much more fast and easy.

The $\mathrm{PhD}$ candidate has studied the potential of capillary columns based on ionic liquids as stationary phases in gas chromatography in order to carry out the determination of chlorobenzenes in soil samples. She also has carried out a novel coupling of in situ derivatization and extraction with the MEPS technique for the determination of chlorophenols in soils.

The candidate has also worked with biological matrices. Due to the importance of the determination of amino acids and other biomarkers in urine for the early detection of different illnesses, new techniques which allow a fast determination of these compounds in biological matrices are urgently needed. In order to improve these methodologies, the candidate has successfully coupled the derivatization process of amino acids with the headspace sampling in order to reduce sample manipulation and reduce the use of solvents and minimize waste.

Also, a methodology using the headspace sampling as pretreatment technique of urine samples without preconcentration step is proposed for the fast determination of possible biomarkers from different chemica families.

In view of the quality of the work presented, the scientific novelty and the real contribution to analytical sciences of the methodologies proposed, I strongly propose this thesis to be accepted in the context of the International PhD Programme of the University of Salamanca.

Informo que la calidad científica de esta Tesis es merecedora de obtener la Mención Doctor Internacional (marcar con una cruz una opción)

I inform that the scientific quality of this thesis is worthy of obtaining the International Doctor Mention (mark with across one option)

\section{Favorable}

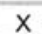

FECHA / DATE : 3rd April 2017

FIRMA / SIGNATURA original:

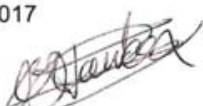

No favorable/Not favorable

Chemistry Research Laboratory

Department of Chemistry

University of Oxford

Mansfield Road

Oxford OX1 3TA

SELLO DE LA INSTITUCIÓN DEL FIRMANTE / THE INSTITUTION'S STAMP OF THE PERSON WHO FIRMS 
